\title{
COMPARISON AND DESIGN OF SIMPLIFIED GENERAL PERTURBATION MODELS (SGP4) AND CODE FOR NASA JOHNSON SPACE CENTER, ORBITAL DEBRIS PROGRAM OFFICE
}

\author{
A Graduate Project \\ presented to \\ the Faculty of California Polytechnic State University, \\ San Luis Obispo
}

\author{
In Partial Fulfillment \\ of the Requirements for the Degree \\ Master of Science in Aerospace Engineering
}

by

Nicholas Zwiep Miura

May 2009 
(C) 2009

Nicholas Zwiep Miura

ALL RIGHTS RESERVED 


\section{COMMITTEE MEMBERSHIP}

TITLE:

AUTHOR:

DATE SUBMITTED:

COMMITTEE CHAIR:

COMMITTEE MEMBER:

COMMITTEE MEMBER: Jordi Puig-Suari, Ph.D

COMMITTEE MEMBER: $\quad$ Edwin Barker, Ph.D
Comparison and Design of Simplified General

Perturbation Models (SGP4) and Code for NASA

Johnson Space Center, Orbital Debris Program Office.

Nicholas Zwiep Miura

May 29, 2009

Kira Abercromby, Ph.D

Eric Mehiel, Ph.D 


\begin{abstract}
Comparison and Design of Simplified General Perturbation Models (SGP4) and Code for NASA Johnson Space Center, Orbital Debris Program Office
\end{abstract}

Nicholas Zwiep Miura

This graduate project compares legacy simplified general perturbation model (SGP4) code developed by NASA Johnson Space Center, Orbital Debris Program Office, to a recent public release of SGP4 code by David Vallado. The legacy code is a subroutine in a larger program named PREDICT, which is used to predict the location of orbital debris in GEO. Direct comparison of the codes showed that the new code yields better results for GEO objects, which are more accurate by orders of magnitude (error in meters rather than kilometers). The public release of SGP4 also provides effective results for LEO and MEO objects on a short time scale. The public release code was debugged and modified to provide instant functionality to the Orbital Debris Program Office. Code is provided in an appendix to this paper along with an accompanying CD. A User's Guide is presented in Chapter 7. 


\section{ACKNOWLEDGMENTS}

I would like to thank my wife, Jesselle, for all her support throughout the year. Also, a special thanks to Dr. Abercromby, my thesis advisor, who provided excellent guidance and resources during the accelerated process. 


\section{TABLE OF CONTENTS}

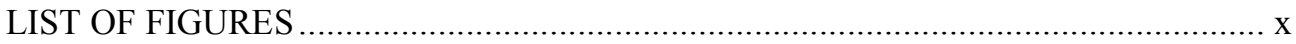

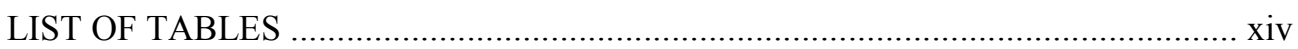

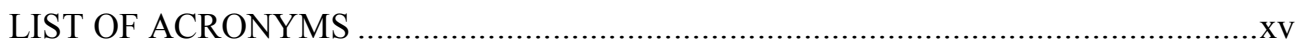

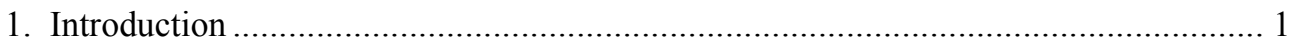

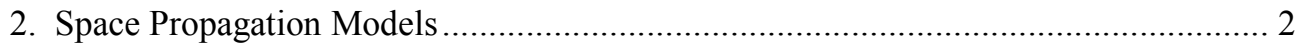

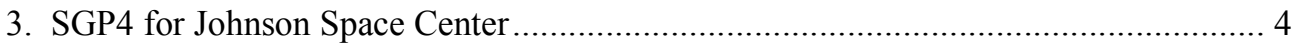

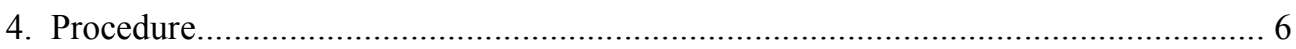

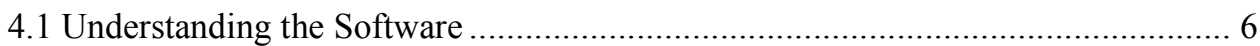

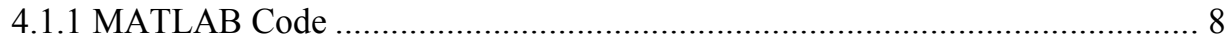

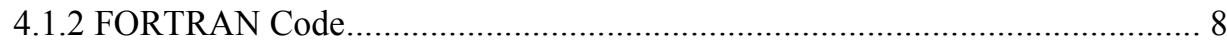

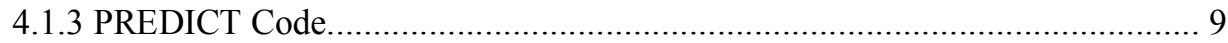

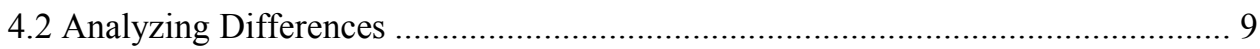

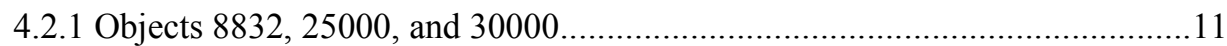

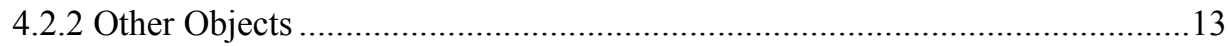

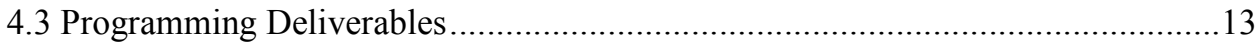

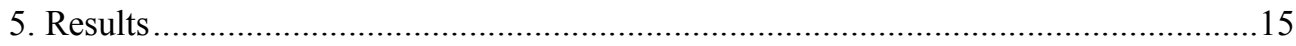

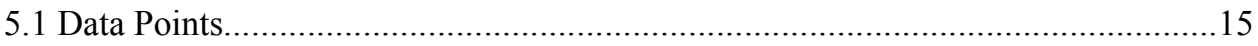

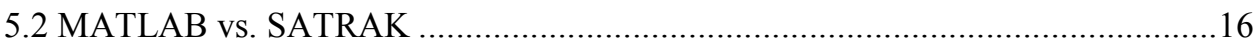

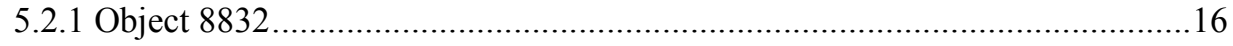

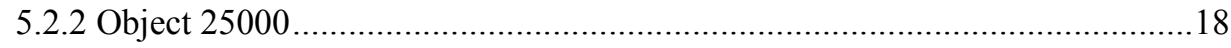

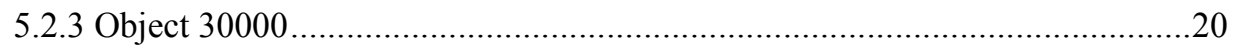

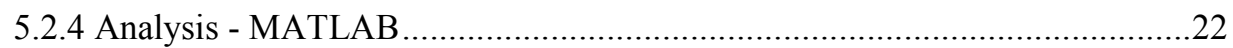




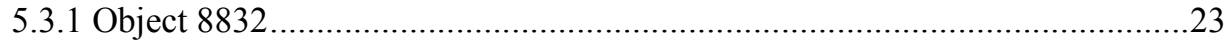

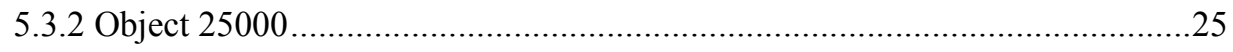

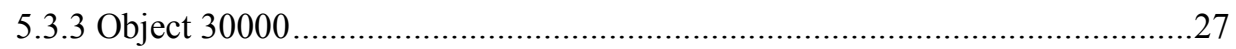

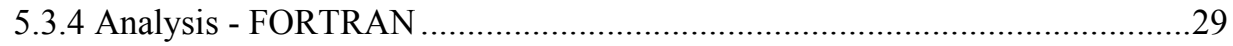

5.4 Modified Code vs. SATRAK ................................................................... 31

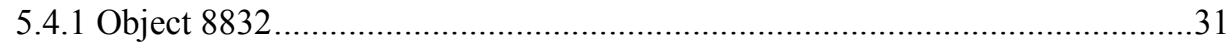

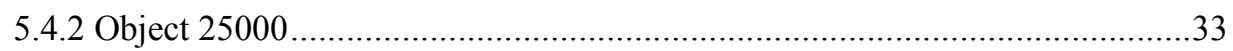

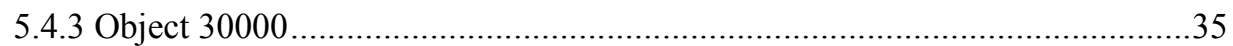

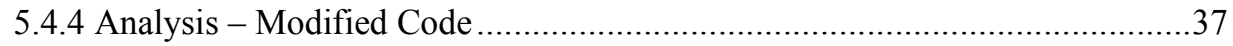

5.5 JSC Code vs. SATRAK ...................................................................... 38

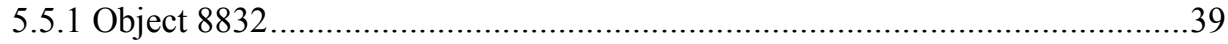

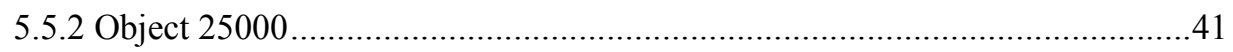

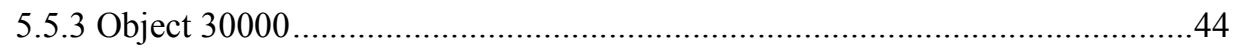

5.5.4 Analysis - JSC Code ........................................................ 45

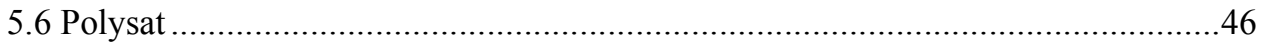

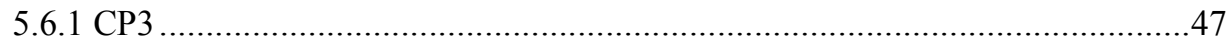

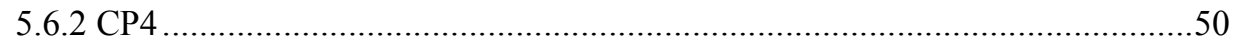

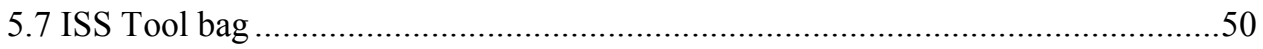

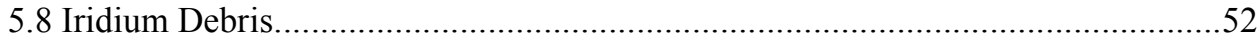

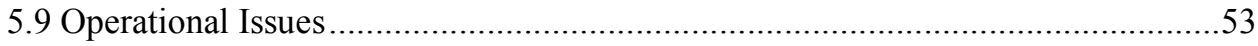

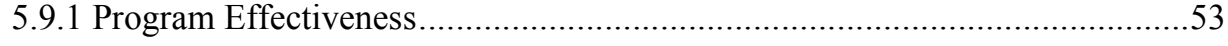

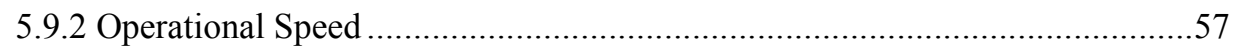




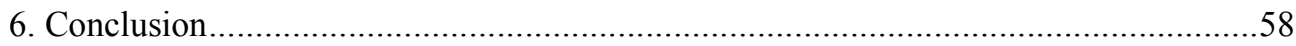

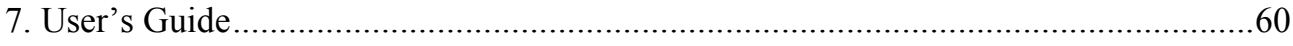

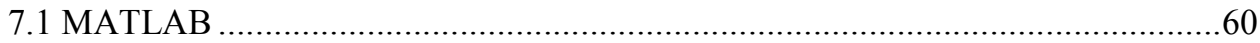

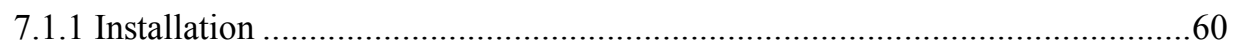

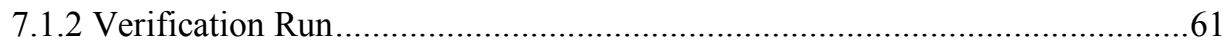

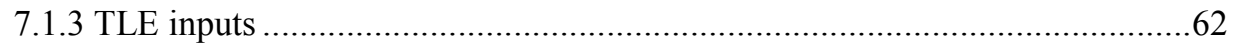

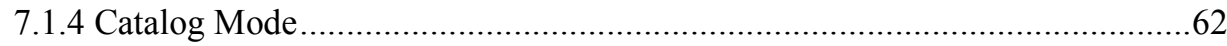

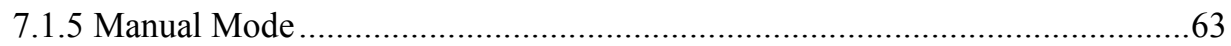

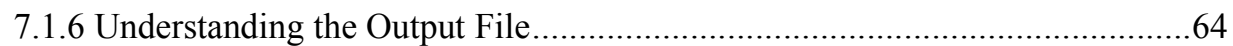

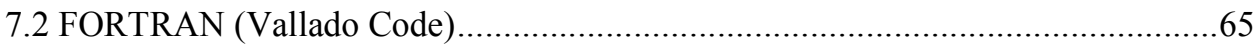

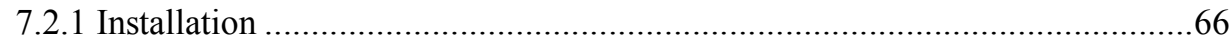

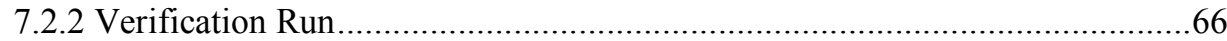

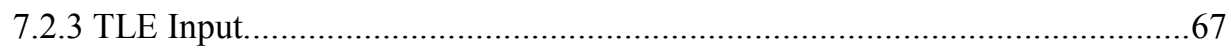

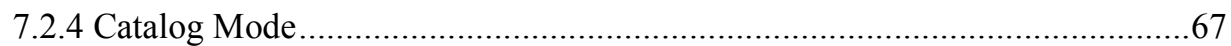

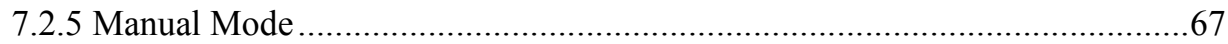

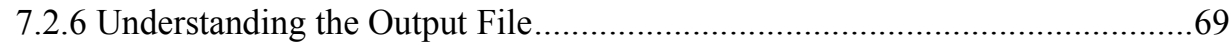

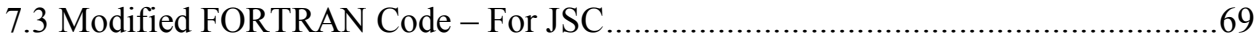

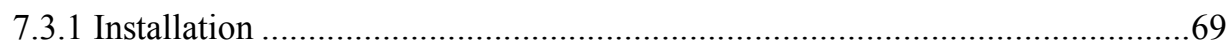

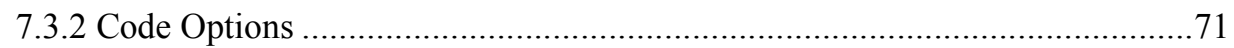

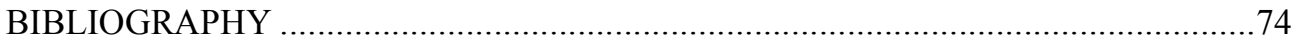

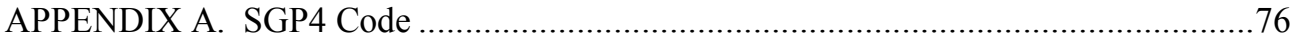

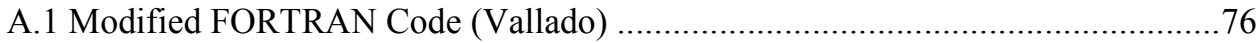

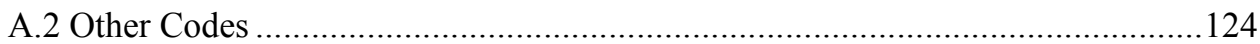




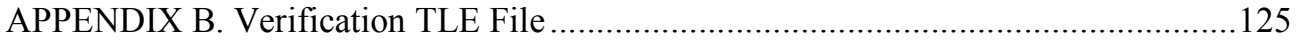

APPENDIX C - Verification Results Data ……......................................................127

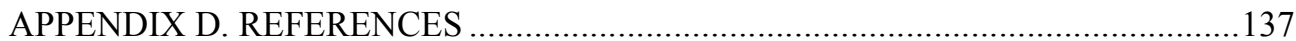




\section{LIST OF FIGURES}

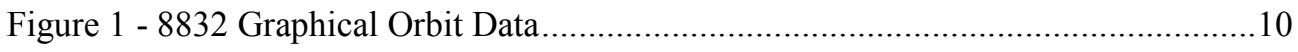

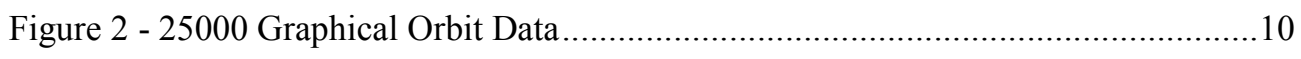

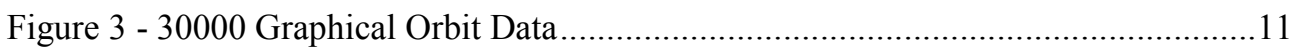

Figure 4 - 8832 Position Error MATLAB (60 min intervals) ........................................16

Figure 5 - 8832 Velocity Error MATLAB (60 min intervals) .......................................17

Figure 6 - 8832 Position Error MATLAB (360 min intervals) ......................................17

Figure 7 - 8832 Velocity Error MATLAB (360 min intervals) ......................................18

Figure 8 - 25000 Position Error MATLAB (60 min intervals) ......................................18

Figure 9 - 25000 Velocity Error MATLAB (60 min interval) .......................................19

Figure 10 - 25000 Position Error MATLAB (360 min interval).....................................19

Figure 11 - 25000 Velocity Error MATLAB (360 min interval) ...................................20

Figure 12 - 30000 Position Error MATLAB (60 min interval) ....................................20

Figure 13 - 30000 Velocity Error MATLAB (60 min interval) ....................................21

Figure 14 - 30000 Position Error MATLAB (360 min intervals) .................................21

Figure 15 - 30000 Velocity Error MATLAB (360 min intervals) .................................22

Figure 16 - Comparison of 60 and 360 minute intervals ..........................................23

Figure 17 - 8832 Position Error FORTRAN (60 min intervals) .....................................24

Figure 18 - 8832 Velocity Error FORTRAN (60 min intervals)...................................24

Figure 19 - 8832 Position Error FORTRAN (360 min intervals).................................25

Figure 20 - 8832 Velocity Error FORTRAN (360 min intervals) ................................2

Figure 21 - 25000 Position Error FORTRAN (60 min intervals) ..................................26

Figure 22 - 25000 Velocity Error FORTRAN (60 min intervals).................................26 
Figure 23 - 25000 Position Error FORTRAN (360 min intervals) ...............................27

Figure 24 - 25000 Velocity Error FORTRAN (60 min intervals)..................................2

Figure 25 - 30000 Position Error FORTRAN (60 min intervals) .................................28

Figure 26 - 30000 Velocity Error FORTRAN (60 min intervals).................................28

Figure 27 - 30000 Position Error FORTRAN (360 min intervals) ................................29

Figure 28 - 30000 Velocity Error FORTRAN (360 min intervals)...............................29

Figure 29 - Comparison of MATLAB and FORTRAN results (8832 position)................30

Figure 30 - Comparison of MATLAB and FORTRAN (8832 Velocity) ...........................31

Figure 31 - 8832 Position Error Vallado (60 min intervals) ...........................................32

Figure 32 - 8832 Velocity Error Vallado (60 min intervals) ..........................................32

Figure 33 - 8832 Position Error Vallado (360 min intervals) .......................................33

Figure 34 - 8832 Velocity Error Vallado (360 min intervals) ........................................33

Figure 35 - 25000 Position Error Vallado (60 min intervals) ............................................34

Figure 36 - 25000 Velocity Error Vallado (60 min intervals) .......................................34

Figure 37 - 25000 Position Error Vallado (360 min intervals) .......................................35

Figure 38 - 25000 Velocity Error Vallado (360 min intervals) .......................................35

Figure 39 - 30000 Position Error Vallado (60 min intervals) ..........................................36

Figure 40 - 30000 Velocity Error Vallado (60 min intervals) .......................................36

Figure 41 - 30000 Position Error Vallado (360 min intervals) .......................................37

Figure 42 - 30000 Velocity Error Vallado (360 min intervals) ........................................37

Figure 43 - Comparison of Vallado to MATLAB (8832 Velocity Error) ..........................38

Figure 44 - 8832 Position Error Matney (60 min intervals) ...........................................39

Figure 45 - 8832 Velocity Error Matney (60 min intervals)..........................................40 
Figure 46 - 8832 Position Error Matney (360 min intervals) .......................................40

Figure 47 - 8832 Velocity Error Matney (360 min intervals) .........................................41

Figure 48 - 25000 Position Error Matney (60 min intervals) ........................................41

Figure 49 - 25000 Velocity Error Matney (60 min intervals) .......................................42

Figure 50 - 25000 Position Error Matney (360 min intervals).......................................42

Figure 51 - 25000 Velocity Error Matney (360 min intervals) .......................................43

Figure 52 - 30000 Position Error Matney (60 min intervals) ........................................44

Figure 53 - 30000 Velocity Error Matney (60 min intervals) ........................................4

Figure 54 - 30000 Position Error Matney (360 min intervals).......................................45

Figure 55 - 30000 Velocity Error Matney (360 min intervals) ......................................45

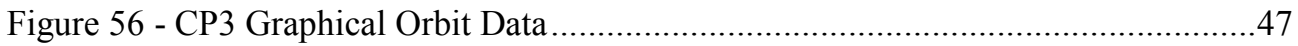

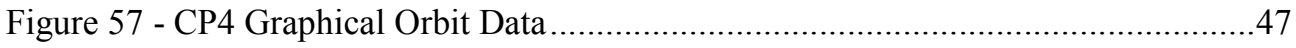

Figure 58 - CP3 Position Error Vallado (10 sec intervals) ..............................................48

Figure 59 - CP3 Velocity Error Vallado (10 sec intervals) …......................................48

Figure 60 - ISS Tool bag Graphical Orbit Data …….................................................51

Figure 61 - Iridium Debris Graphical Orbit Data........................................................52

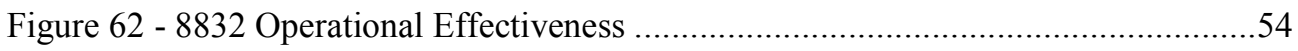

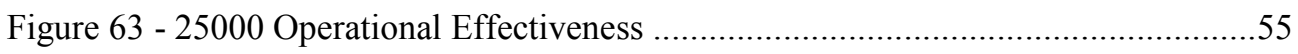

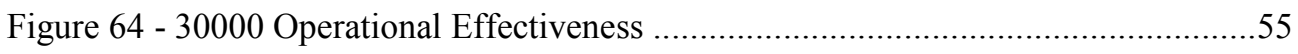

Figure 65 - 30000 Operational Effectiveness (new code) ……......................................56

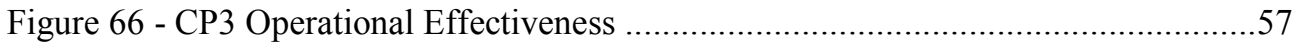

Figure 67 - Code Addition to SURVEYCHASENEW................................................. 70

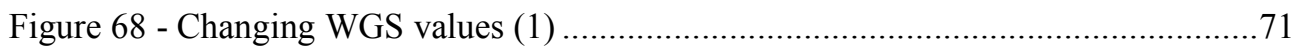




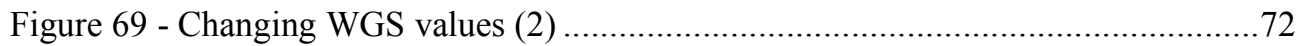

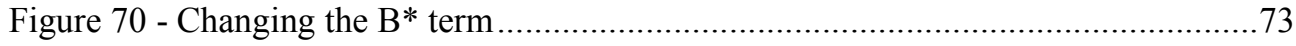




\section{LIST OF TABLES}

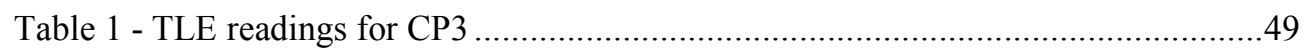

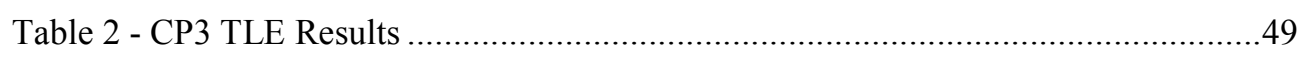

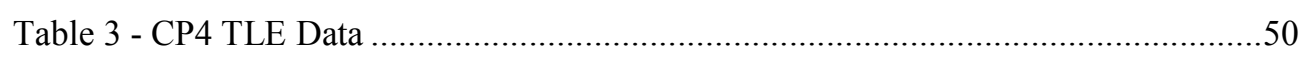

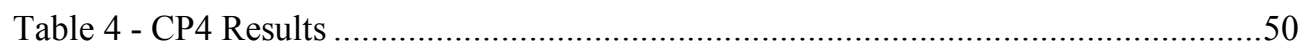

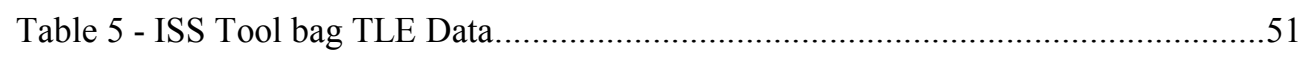

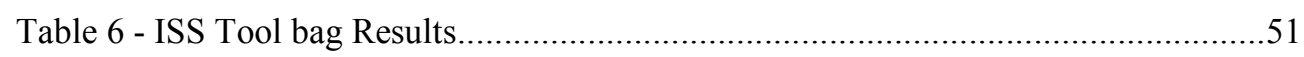

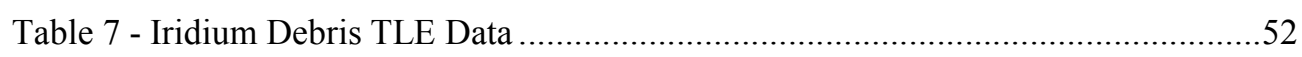

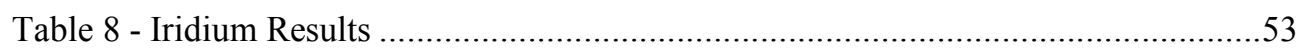




\section{LIST OF ACRONYMS}

NASA

SGP(4)

PREDICT

SPACETRACK

AIAA

JSC

SATRAK

GSFC

JPL

FORTRAN

COES

TLE

WGS

LES

GEO

LEO

MEO

ISS

$\min$

$\mathrm{m}$

$\mathrm{cm}$

$\mathrm{mm}$

sec

$\mathrm{km}$

Polysat

CP3

CP4

FOV
National Aeronautics and Space Administration

Simplified General Perturbation

NASA code that predicts position of orbital debris

Government program initiated to track satellites

American Institute of Aeronautics and Astronautics

Johnson Space Center

Satellite Tracking (Government owned propagator)

Goddard Space Flight Center

Jet Propulsion Laboratory

Formula Translating (IBM developed language)

Classical Orbital Element Set

Two Line Element

World Geodetic System

Lincoln Experimental Satellite

Geosynchronous Earth Orbit

Low Earth Orbit

Medium Earth Orbit

International Space Station

minutes

meters

centimeters

millimeters

seconds

kilometers

Cube satellites developed by Cal Poly

Polysat 3

Polysat 4

Field of view 


\section{Introduction}

This project has been completed for NASA Johnson Space Center, Orbital Debris Program Office, with the purpose of comparing the effectiveness of legacy SGP4 propagation code and a recent public release of SGP4 propagation code. Furthermore, new SGP4 propagation code based on the public release was developed and delivered.

The Orbital Debris Program Offices uses a program called PREDICT to predict the motion of deep space debris. The program was developed in-house and uses a simplified version of Simplified General Perturbation Theory to propagate the position and velocity of a spacecraft forward in time based on classic orbital elements. In 2004, there was a public release of Simplified General Perturbation 4 (SGP4) code, based on the same theory. The Orbital Debris Program Office wanted to know how their legacy code compared with the public release.

This project compares the two codes to truth data provided by the Orbital Debris Program Office, and shows the accuracy of the delivered results. Additionally, the public release code was debugged and provided for use and the public release code was modified to run within the PREDICT program.

This paper describes the problems involved in this undertaking, as well as the procedure taken to solve these problems. Next, a detailed display of the results plus analysis and conclusions is given. Finally, a User's Guide is provided to help navigate using the public release code and to help update the legacy code. 


\section{Space Propagation Models}

Space propagation models use current state information of a satellite to predict a future state of the satellite. As a simplistic example, imagine a car driving down a highway. If we know the location and the speed of the car now, we can make an accurate prediction of where the car will be in an hour. Similarly for satellites, if we know the position and the velocity now, we can make a reasonable guess where the satellite will be in the future. The satellite however, encounters disturbances, or perturbations, along its path that complicates its motion. These perturbations are caused by the Earth's shape (spherical harmonics), drag, radiation, and effects from other bodies (the sun and moon generally). In our car example, imagine driving up and down hills, over different terrain, with changing speed limits. It makes our simplified example much harder.

Space propagation models are used primarily by agencies that track orbiting objects. The ultimate goal is to know where everything in space is at all times. Propagation models are needed because there are simply too few telescopes to watch everything in the sky at all times. Thus, you find the position and velocity of an object once, and then use the propagator to tell you where it is in the future if you ever need to locate it again. The first attempt to use these models came in 1959, by the National Space Surveillance Control Center. This was a highly simplified model that failed when trying to propagate position more than a week. In 1960, the initial model was replaced by the 'Simplified General Perturbations (SGP)' model ${ }^{\mathrm{ii}}$. Though this model provided accurate results, by 1969 there were too many satellite in orbit to maintain a catalog based on SGP. SGP4, published in SPACETRACK Report \#3, was created based on a simplified version of 
SGP. By 1979, it was the sole model for catalog maintenance. SGP4 failed when objects were in 'deep space' orbits, so in 1977, an extension to the program was developed to track object with an altitude over $255 \mathrm{~km}$ - the height where solar/lunar perturbations have a larger effect than atmospheric drag.

After SPACETRAK Report \#3 was released, the 'official' code maintained by the U.S. government became an export controlled black-box. Changes to the code to reflect new programming techniques, better computing power, and more accurate constants were never made public; instead, were made available to select agencies through an executable program called SATRAK.

Agencies not privy to SATRAK, or who needed customized code for their project (including NASA) were forced to manipulate the original code found in SPACETRAK Report \#3 themselves, leading to a wide variety of propagation codes based on the same model. David Vallado iii, working through the Center for Space, released an AIAA paper in 2004, which attempted to reconcile the many codes into one standardized code. This new code was made available to the public through celestrak.com. 


\section{SGP4 for Johnson Space Center}

Johnson Space Center (JSC) - Orbital Debris Program Office was one project that required the use of a Space Propagation Model. Though they had access to an executable version of SATRAK, customized code was needed to run within a larger program called PREDICT. A telescope observes a debris object and takes right ascension and declination data over a short period of time. The PREDICT code then takes this data and formulates a classical orbital element set (COES) that is fed into the space propagation model. The model can then estimate where the object will be in the near future so it can be found again and analyzed further.

Dr. Mark Matney of the Orbital Debris Program Office was the original architect of the PREDICT space propagation model in 1998. Dr. Matney based his work on the original SPACETRACK Report \#3 as well as a publicly released SGP4 model from Goddard Space Flight Center (1997). Though Dr. Matney's program outputs workable results, the Orbital Debris Program Office was intrigued by Vallado's standardized code and wanted to explore the differences between codes. Furthermore, Dr. Matney's code did not accurately predict the location of the objects in some instances, though specific cases were not supplied for analysis.

This project, made possible through a research contract between JSC and Cal Poly San Luis Obispo, explores the differences in the two propagation codes. The scope of the project is as follows:

- Quantify the errors in the two codes based on 'truth' data. 
- Deliver new PREDICT code integrated with updated SGP4 module.

- Deliver SGP4 propagator in MATLAB.

All analysis and deliverables are contained within this final report, as well as user guides for attached software code. 


\section{Procedure}

This project was divided into three distinct stages.

1. Understanding the software.

2. Analyzing differences.

3. Programming deliverables.

\subsection{Understanding the Software}

The starting point of this phase was David Vallado's (et al) AIAA paper, Revisiting ${\underline{\text { Spacetrack Report } \# 3^{\text {iv }}}}$. In this paper, updates to the code are described in detail, and a link to the code in both FORTRAN and MATLAB is available at:

$\underline{\text { http://CelesTrak.com/software/vallado-sw.asp }}$

In Vallado's analysis, results from the new code are compared against selected results from existing public code (i.e. GSFC, JPL, T.S. Kelso's FORTRAN). Though there are obvious differences, there is no proof that the new code is closer or further from the truth. What the paper does do is analyze each difference and explain why the results differ. These differences are commented as 'fixes' within the code and are cataloged in detail in Vallado's paper.

The code that Vallado created first prompts users for an operations mode. This selects how the code calculates epoch of the moon and sun for lunar/solar perturbations. Next, 
the code prompts users to input the type of run. Of the three choices, the first is catalog mode, where the code displays position, velocity, and COES for 12 hours forwards and backwards in time with a step of 20 minutes. Second is verification mode, designed to test the output of a given two line element (TLE) set and verify the results against a given file. Third is manual mode, where users define the propagation parameters. If manual mode is chosen, the user gets to determine if they want to use minutes from epoch, epoch, or day of year approach to define the window of time to analyze. The code itself converts epoch and day of year inputs to minutes from epoch when calling the propagator. Next, the user chooses which Earth constants to use, WGS-72 or WGS-84 referring to the World Geodetic System constants and when they became standard. Though the WGS-84 numbers are the current standard (through 2010), WGS-72 values are used in most SGP4 propagators because the 1984 values were not available at the time of creation. Though it is unknown what values SATRAK uses, analysis shows that WGS-72 numbers give more accurate results than WGS-84. Finally, the code calls for TLE inputs. The TLE file must be in the same directory as the main program, and the format of the TLE must be congruent with standards. Multiple TLEs may be in the same file with non TLE lines marked with a '\#' symbol. The program then opens or creates an OUT file and prints the data. The OUT file has a header line containing the satellite number. Following lines are in the following form: a column for minutes from epoch, three columns of position data, three columns of velocity data, and four columns of date and time, delineated by a space. 


\subsubsection{MATLAB Code}

The MATLAB code provided by Vallado contained some bugs. There were two major issues encountered. The first involved calling an undefined subroutine. The code tried to call a subroutine called 'days $2 \mathrm{mdhms}$ ', but the subroutine needed was named 'days2mdh'. This subroutine had inputs of the year and day of year, and output the month, day, hour, minute, and second. After this bugged was corrected, another problem was encountered with time. The propagator uses minutes since epoch as an input, but instead was receiving days since epoch. This bug was corrected by dividing critical numbers by 1440 (minutes in a day), and reformatting the output to reflect changes. This code was tested in this debugged form and will be delivered to NASA in its current form to be used as an alternative to SATRAK.

\subsubsection{FORTRAN Code}

Before working on the FORTRAN code a compiler was needed. The chosen compiler was a free compiler available online called Silverfrost.

(http://download.cnet.com/Silverfrost-FTN95/3000-2069 4-10491439.html)

The code is written in fixed-format FORTRAN 90 language. Additionally, the complete code was spread amongst 25 small .FOR files and 4 common files upon initial download. Based on my understanding of the MATLAB code, I was able to collect all the relevant subroutines and create a new .FOR file that provided the same functionality as the MATLAB code. The code itself did not have any major bugs like the MATLAB code; however, significant time was spent formatting the combined code to fit the fixed-format FORTRAN standards. 


\subsubsection{PREDICT Code}

JSC Orbital Debris Program Office provided their PREDICT code written in FORTRAN. The code is written as a series of subroutines. Though the focus was directed towards the SGP4 subroutine, the interactions with other parts of the program also needed to be taken into account. The SGP4 subroutine is called with a COES input and a time from epoch (when the COES was taken). The output is a single position and velocity vector based on the time from epoch. The code itself is relatively simplistic. It only takes into account J2 perturbations (the first mode of Earth's spherical harmonics), and then uses Kepler's solution with Newton's method to solve for position and velocity.

\subsection{Analyzing Differences}

JSC had three distinct test cases to analyze that were all debris in geosynchronous orbits. For these cases $(8832,25000$, and 30000), JSC sent a TLE and SATRAK truth data. The truth data consists of a data set with 60 minute intervals between predictions and a data set with 360 minute intervals between predictions - but with a larger time range. These objects are of particular importance to the Orbital Debris. All three objects are pieces of debris from a Titan $3 \mathrm{C}$ transtage launch vehicle. The pieces themselves are not exactly in GEO, but are relatively close in a subGEO orbit. Below describes the break-up of objects 25000 and 30000.

The breakup of the Titan 3C-4 transtage occurred on 21February 1992 at an altitude of $\sim 35,600 \mathrm{~km}$, inclination (INC) of 11.9 degrees and a right ascension (RA) of 21.8 hours. The operator of the GEODSS sensor on 
Maui, Hawaii witnessed approximately 20 pieces in the breakup, but none were tracked at the time. Subsequent to the breakup, the U. S. Space Surveillance Network (SSN) identified three pieces of the debris and assigned them to the catalogue as SSN25000, SSN25001 and SSN30000. ${ }^{\mathrm{v}}$
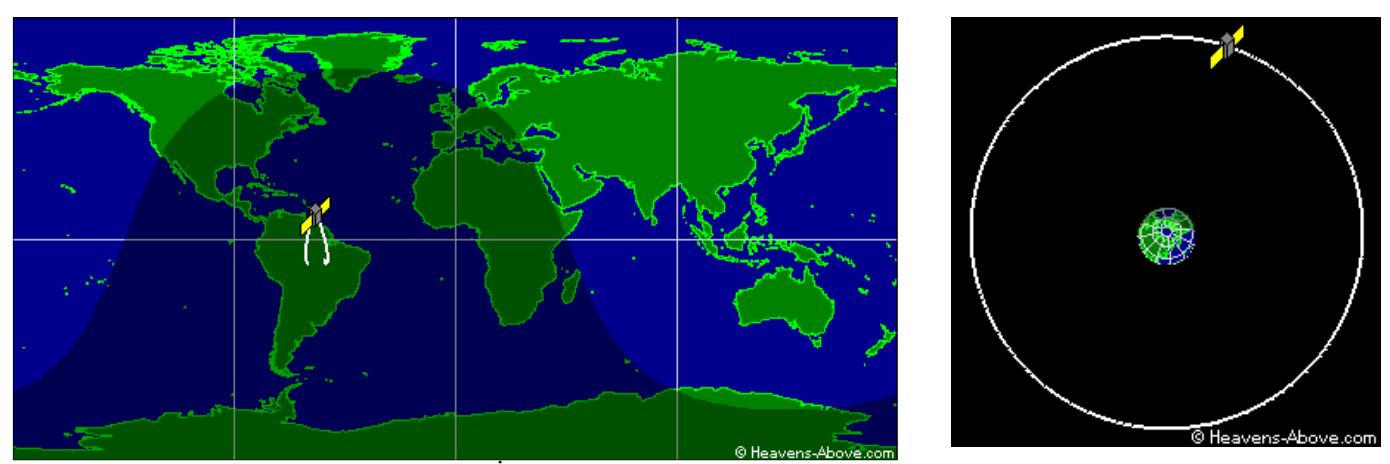

Figure 1 - 8832 Graphical Orbit Data
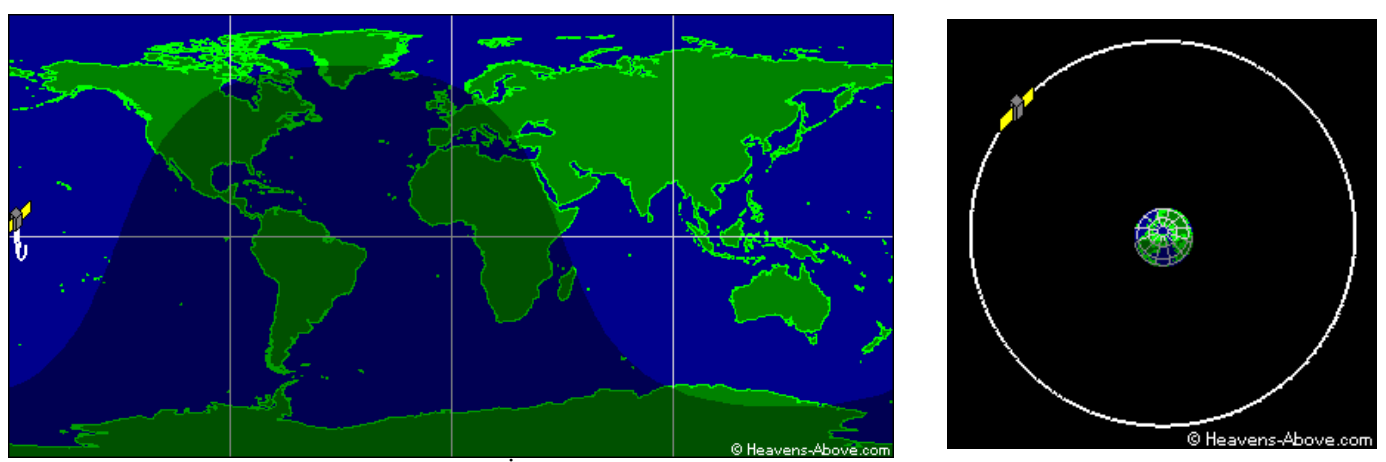

Figure 2 - 25000 Graphical Orbit Data ${ }^{\text {vi }}$ 

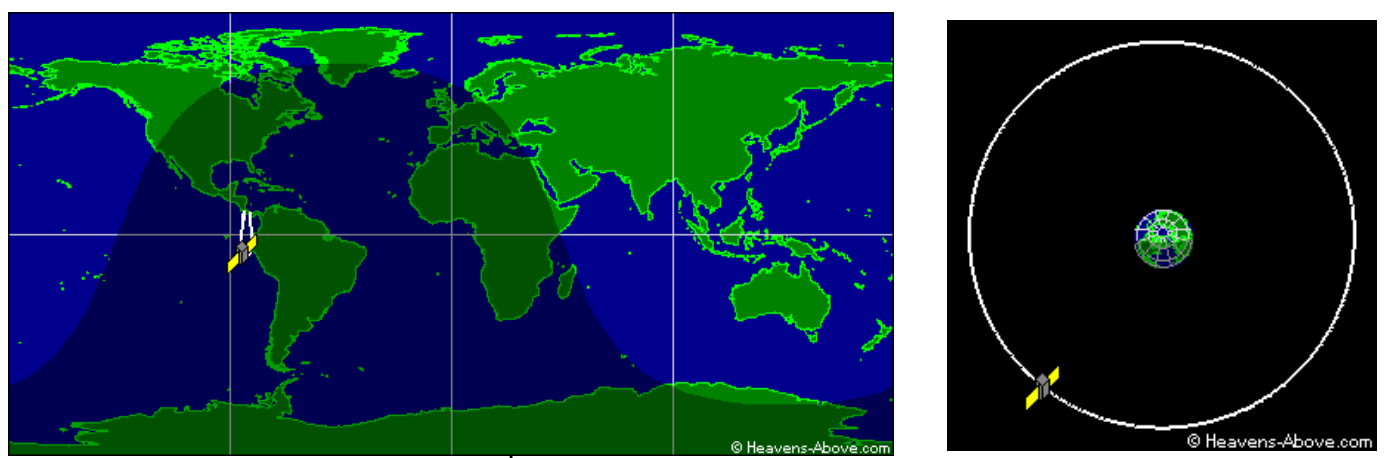

Figure 3 - 30000 Graphical Orbit Data ${ }^{\text {vi }}$

Next, to further the analysis, TLEs were gathered from Celestrak for non-geosynchronous orbits. Then, the same TLEs were again gathered on a later date. Analysis then compared where the object is based on the latter TLE, with where the object was predicted to be based on the former TLE. Analysis was completed on both the MATLAB and FORTRAN codes, as well as JSC's code.

\subsubsection{Objects 8832,25000 , and 30000}

First, each TLE was processed through the MATLAB and FORTRAN versions of Vallado's code and compared side by side with the SATRAK data. The MATLAB and FORTRAN codes can be programmed to have the same output as the SATRAK data. Results were first compared by hand, and then further analyzed using Excel. Error between SATRAK and Vallado's code is quantified as the absolute magnitudes of difference in the position and velocity vectors.

\subsubsection{Code Modification}

Since the ultimate goal of the project is to replace the current SGP4 propagator in JSC's code, the next step was to modify the FORTRAN code to act as a subroutine, instead of a 
stand-alone executable, with the same input and output variables as the original PREDICT model. Because the PREDICT model called for less functionality than the new code provided, much of the work done came in deleting unnecessary lines of code. New lines of code were added to remove any prompts given to the user; however, because the new code can accept LEO objects, a B* prompt was added to the process. There are comments within the new code with instructions on how to remove this if the user does not find it necessary.

Next, a simple program was created in which the user defines the COES from the TLE and gives a 'minutes from epoch' value. The program then calls the propagator and prints the expected position and velocity on the screen.

At this point, the inputs and outputs to the FORTRAN propagator and the PREDICT propagator were the same, and it was trivial to copy and paste the SGP4 subroutine from PREDICT into a new program called by the same simple program from above.

\subsubsection{SATRAK Comparison}

The objects given by JSC were also run through the modified program. However, since the new programs were restrictive in their input and output, only select data points were analyzed until trends were discovered. 


\subsubsection{Other Objects}

To complete the analysis, truth data for LEO and MEO objects were found on SATRAK.

These objects were:

- Polysat CP3 (31129)

- Polysat CP4 (31132)

- Iridium 33 Debris (33771)

- $\operatorname{LES} 9(08747)$

- ISS Tool bag (33442)

The analysis was completed using the modified code because of the single input and

output. Error from truth data is again expressed as the absolute magnitude differences in position and velocity vectors.

\subsection{Programming Deliverables}

The final step of the procedure was installing the new code into the original PREDICT code. The majority of the leg work had already been completed in the analysis phase, though there were still some issues to be resolved. First, some of the variable and subroutine names needed to be altered to work with the PREDICT code. Specifically, both the PREDICT code and the new code have a subroutine labeled 'JDAY'.

Next, through analysis, it was found that the replacement code needs an extra bit of data to work properly. The PREDICT code only looks at J2 effects, which use a statistical 
average of spherical harmonic forces to determine perturbations. This makes epoch data irrelevant because it doesn't matter when you start your calculation, it only matters how long the $\mathrm{J} 2$ force has been acting on the object. Thus, when the propagator is called in the PREDICT model, it only inputs time from epoch and not the epoch itself. In contrast, the new code not only takes into account $\mathrm{J} 2$ effects, but also looks at forces on the object from lunar and solar influences. These effects are heavily dependent on epoch data because the location of the moon and sun need to be known to correctly calculate the direction and magnitude of the forces.

Epoch data for objects analyzed by the PREDICT code is determined in another subroutine, which is a predecessor for the SGP4 subroutine. To solve the problem, a global common file was created to pass the necessary data to the new SGP4 routine. Details of this can be found in comments in the code and in the User's Guide portion of this report.

Complete testing of the PREDICT code with the modified SGP4 code will be completed by the Orbital Debris Program Office. 


\section{Results}

Sections 5.2 through 5.5 graphically display results that represent the magnitude of the difference in position and velocity vectors between the output of various SGP propagators and SATRAK output over the time span defined by given SATRAK truth data. The given time span ranges from $\sim 15$ days to $\sim 27$ days. Each section highlights results from the four main codes that were generated from this project. Furthermore, analysis of the results are presented after each section.

Sections 5.6 through 5.8 show the results of the four objects not in GEO orbit, with truth data taken from the TLE data provided by Celestrak.

Section 5.9 links the results to real-world application.

\subsection{Data Points}

All results were generated from TLEs for the following objects:

- 8832 (Titan debris)

- 25000 (Titan debris)

- 30000 (Titan debris)

- 31129 (Poly Sat 3)

- 31132 (Poly Sat 4)

- 33771 (Iridium 33 debris)

- 33442 (ISS tool bag debris) 


\subsection{MATLAB vs. SATRAK}

Results are displayed for both scenarios created in SATRAK (60 and 360 minute intervals).

\subsubsection{Object 8832}

The maximum position error calculated between SATRAK and the MATLAB results is $32 \mathrm{~cm}$ and the maximum velocity error calculated is $0.023 \mathrm{~mm} / \mathrm{sec}$.

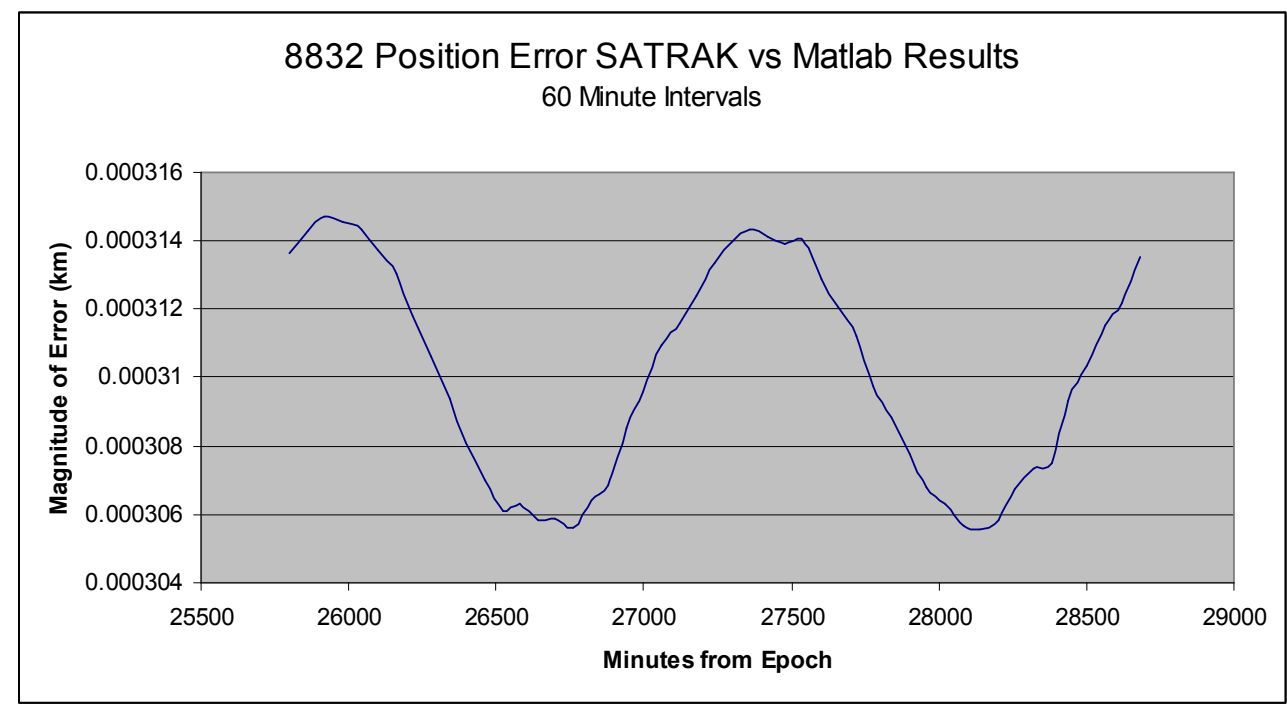

Figure 4 - 8832 Position Error MATLAB (60 min intervals) 


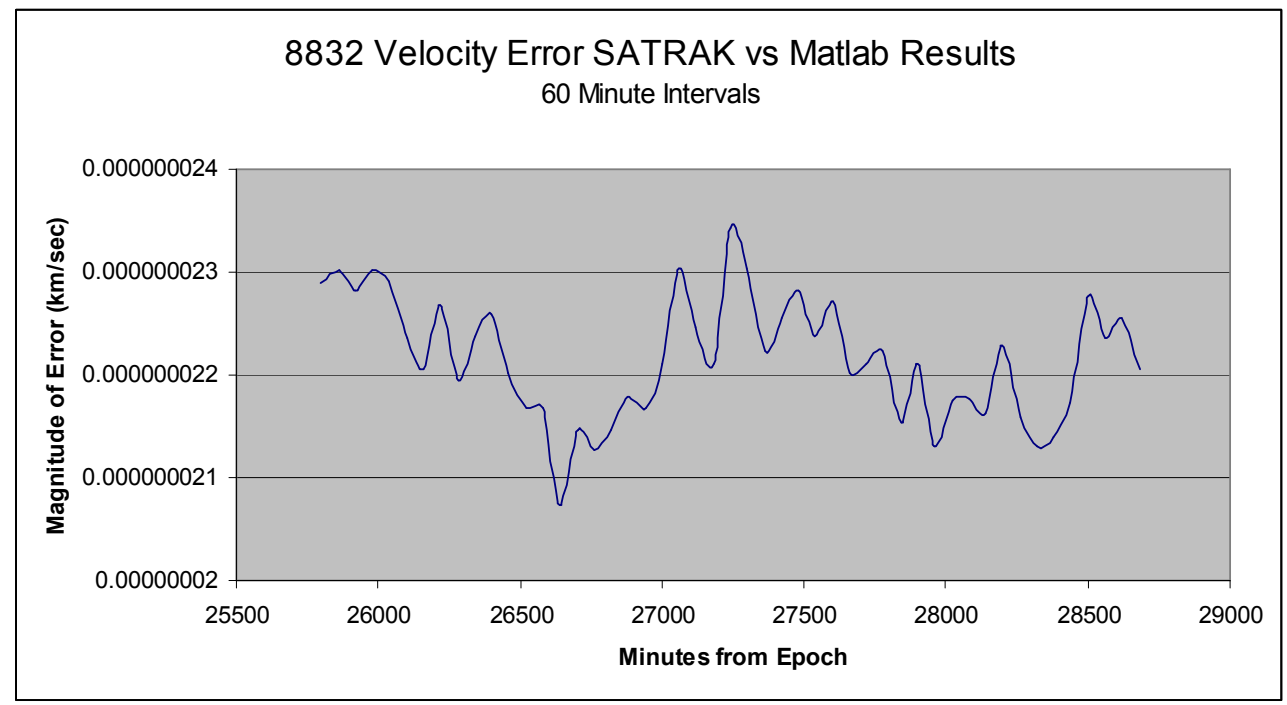

Figure 5 - 8832 Velocity Error MATLAB (60 min intervals)

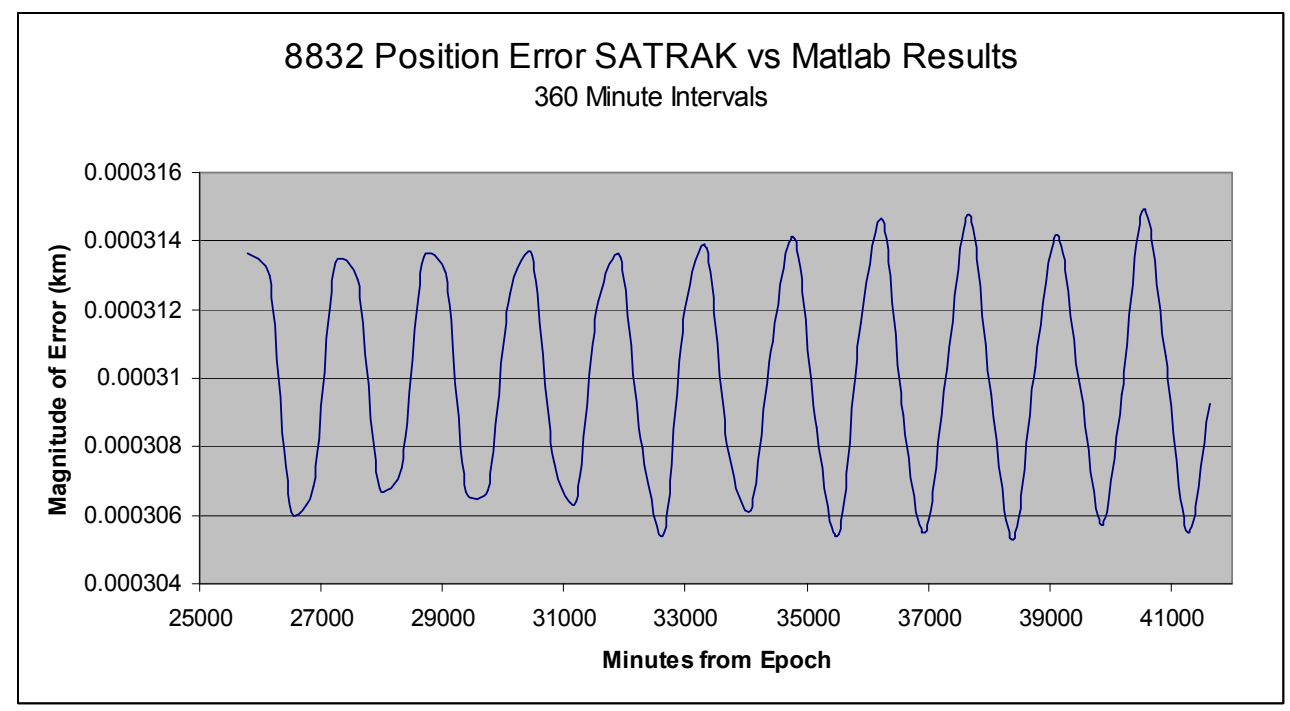

Figure 6 - 8832 Position Error MATLAB (360 min intervals) 


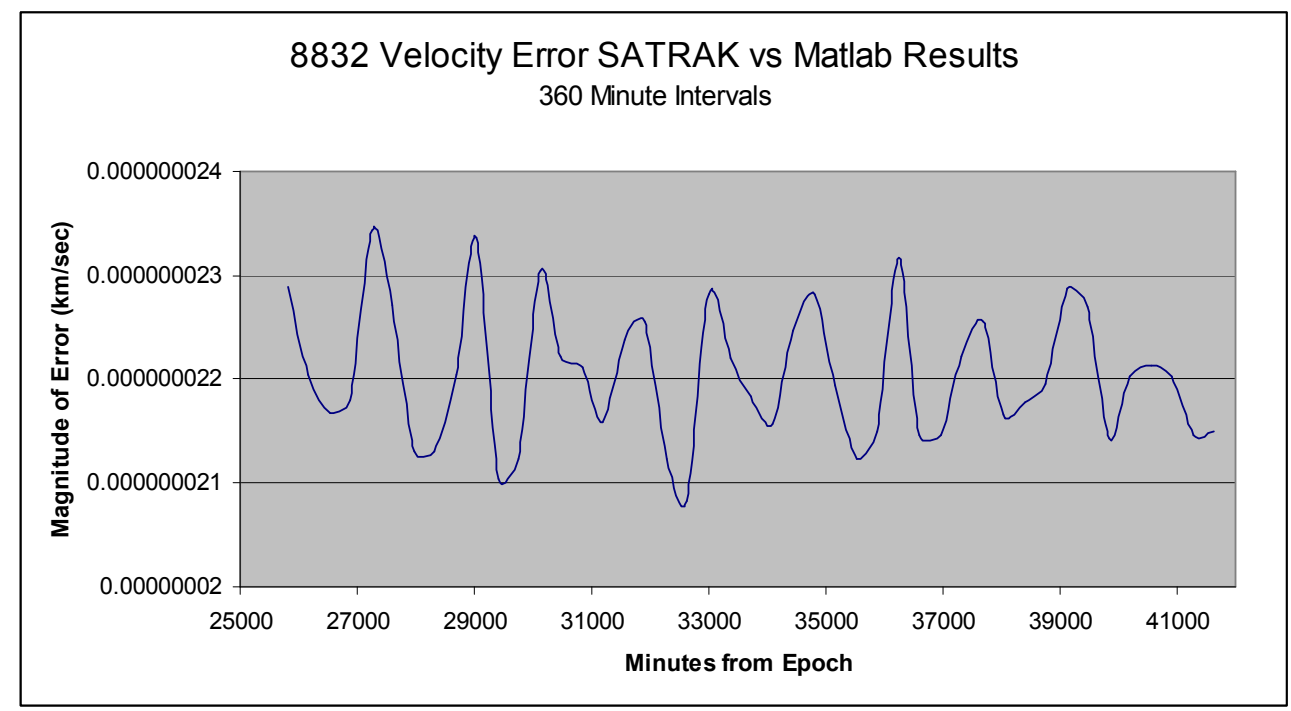

Figure 7 - 8832 Velocity Error MATLAB (360 min intervals)

\subsubsection{Object 25000}

The maximum position error calculated between the SATRAK and MATLAB results is $1.5 \mathrm{~m}$ and the maximum velocity error calculated is $.11 \mathrm{~mm} / \mathrm{sec}$.

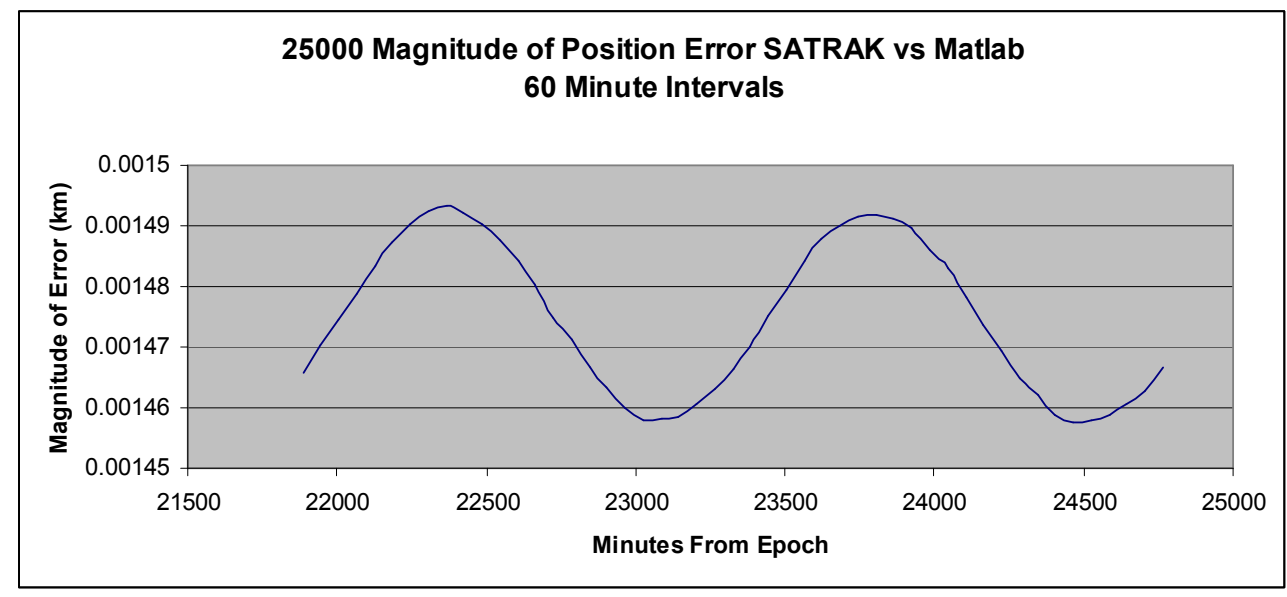

Figure 8 - 25000 Position Error MATLAB (60 min intervals) 


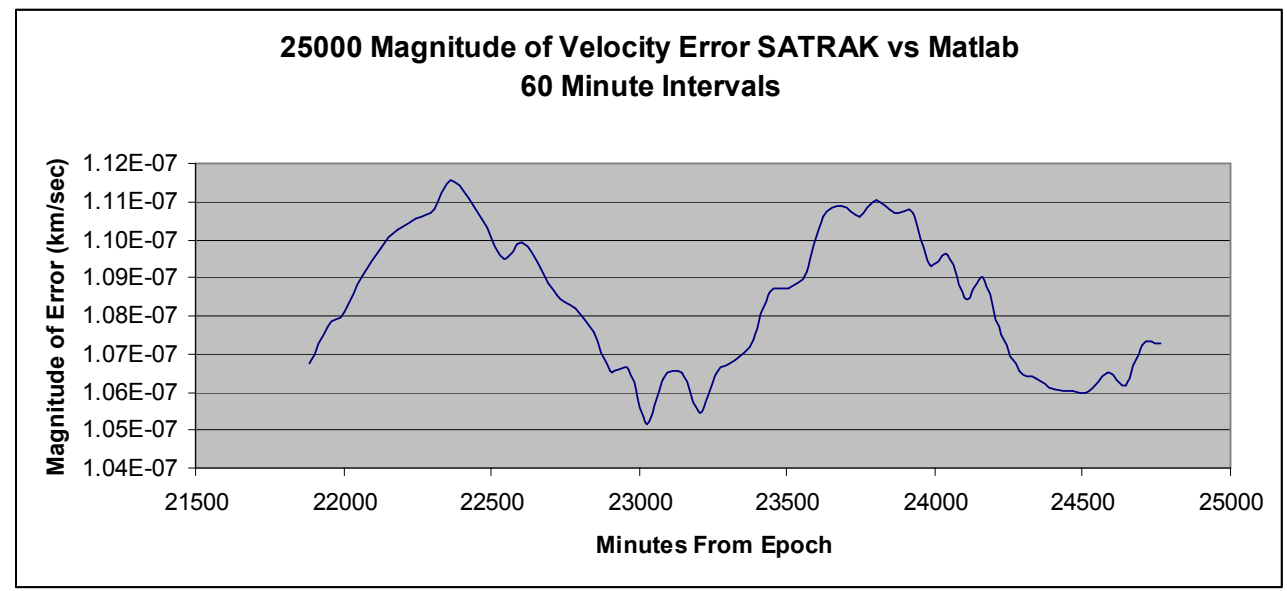

Figure 9 - 25000 Velocity Error MATLAB (60 min interval)

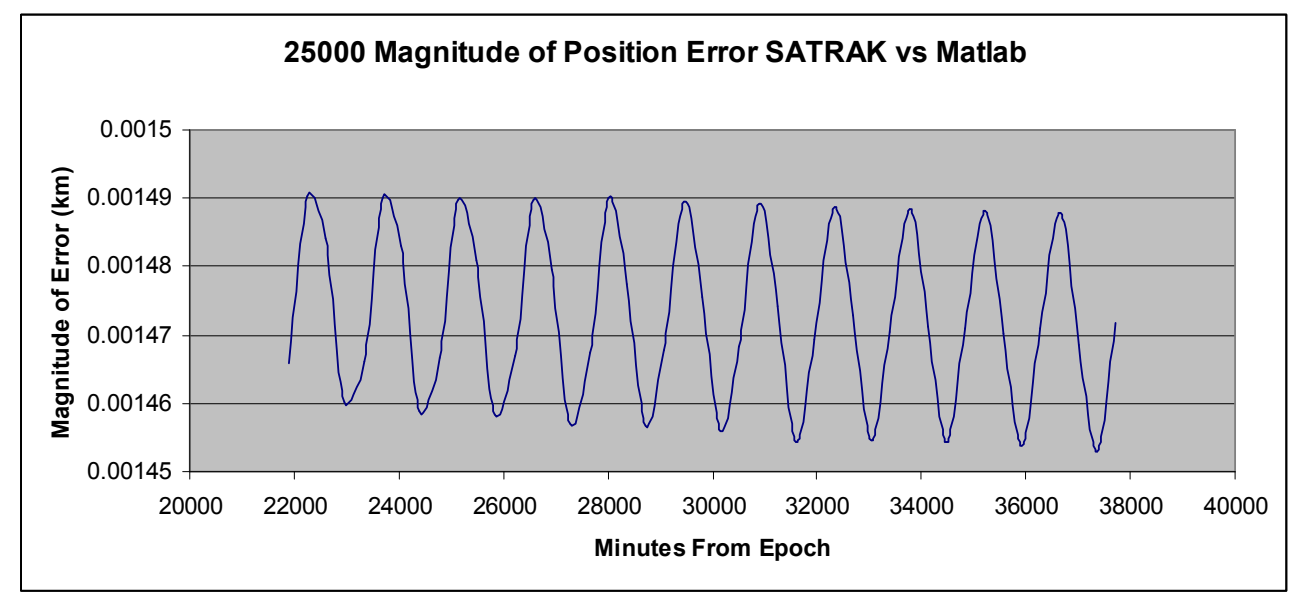

Figure 10 - 25000 Position Error MATLAB (360 min interval) 


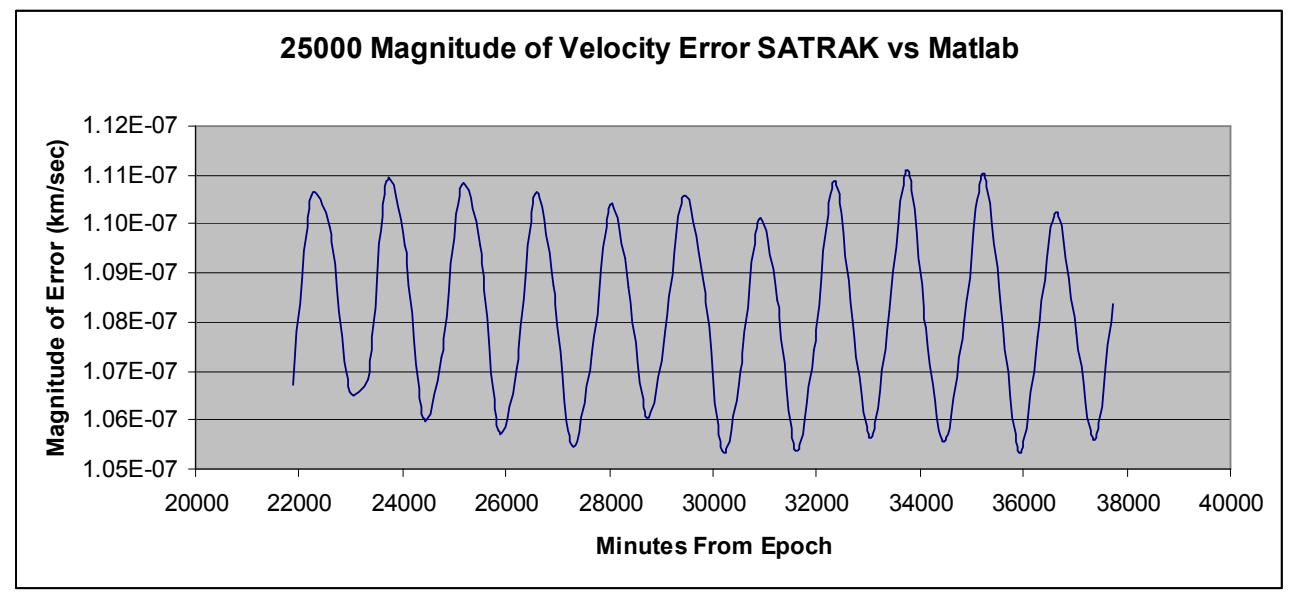

Figure 11 - 25000 Velocity Error MATLAB (360 min interval)

\subsubsection{Object 30000}

The maximum position error calculated is $51 \mathrm{~cm}$ and the maximum velocity error calculated is $0.038 \mathrm{~mm} / \mathrm{sec}$.

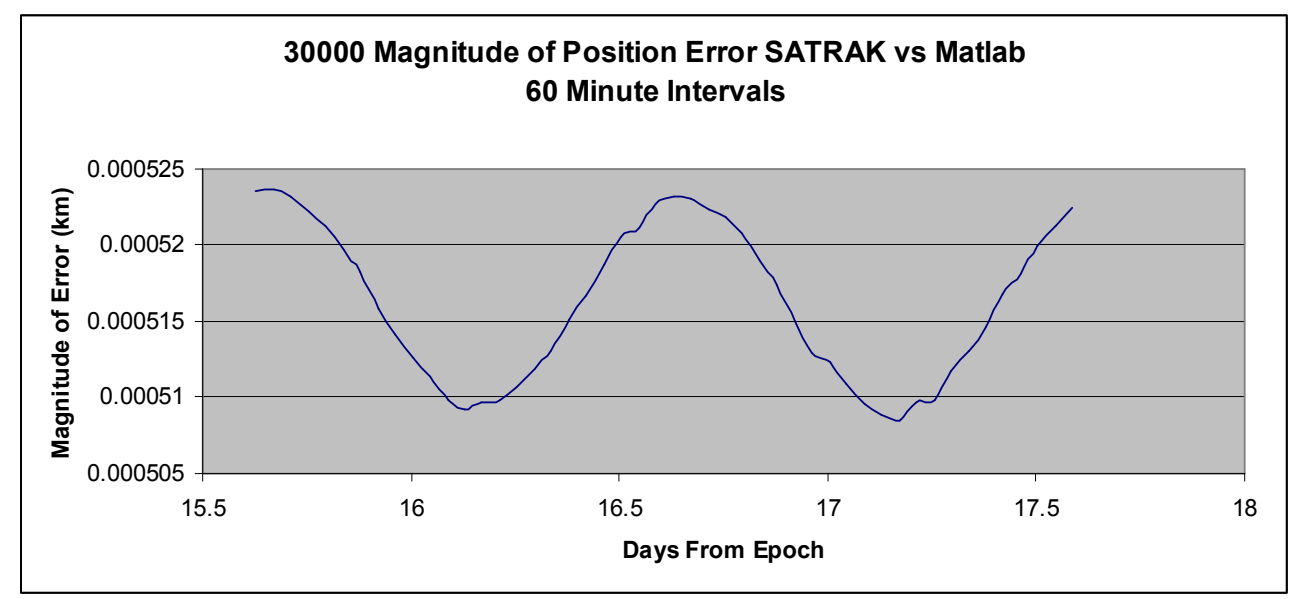

Figure 12 - 30000 Position Error MATLAB (60 min interval) 


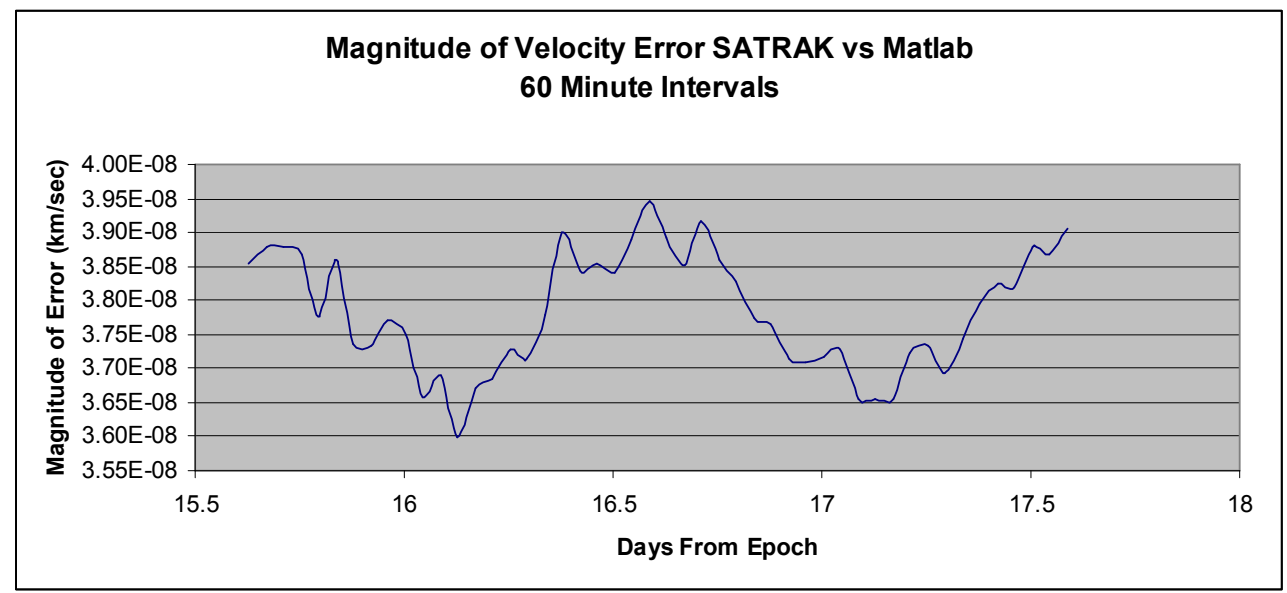

Figure 13 - 30000 Velocity Error MATLAB (60 min interval)

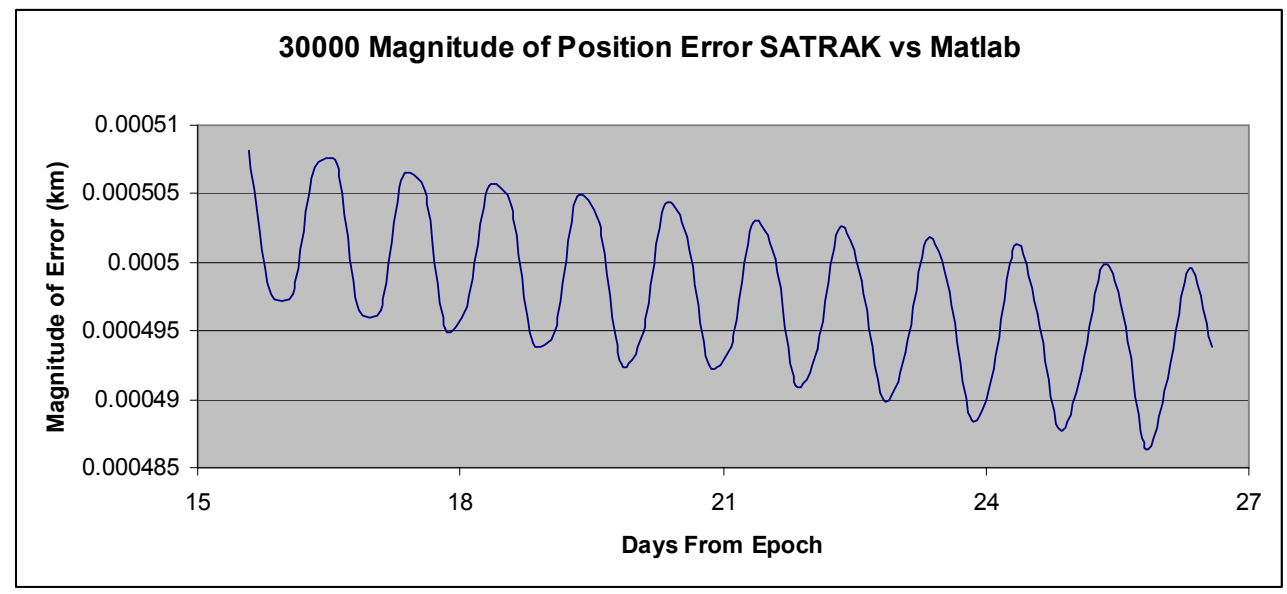

Figure 14 - 30000 Position Error MATLAB (360 min intervals) 


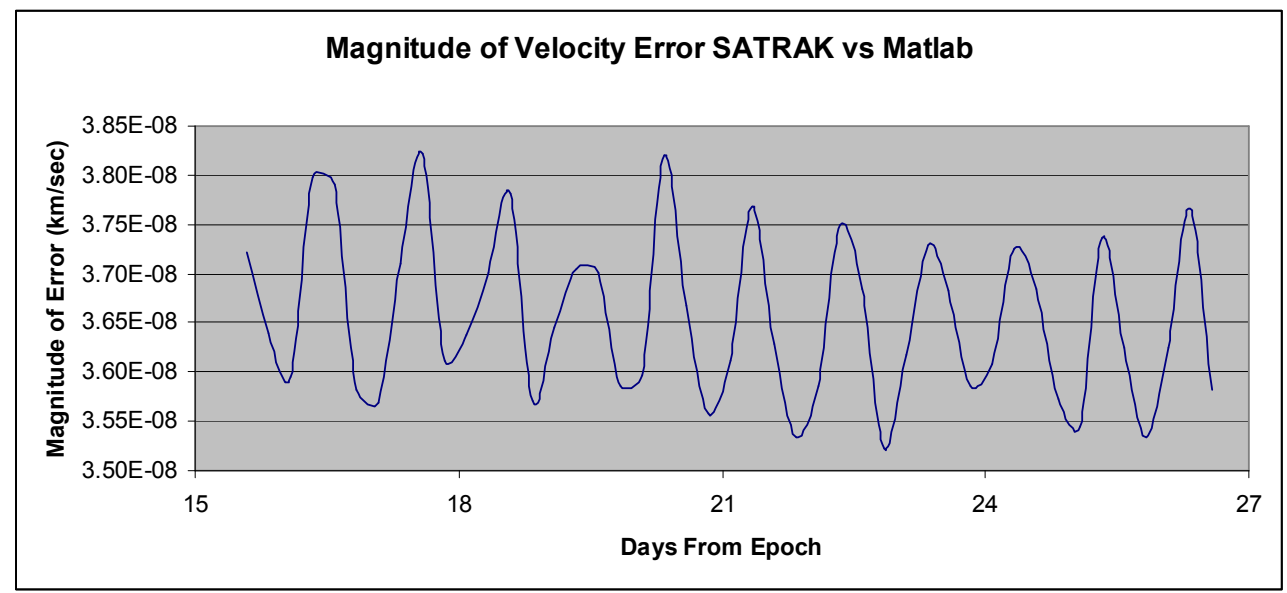

Figure 15 - 30000 Velocity Error MATLAB (360 min intervals)

\subsubsection{Analysis - MATLAB}

In a broad sense, the error between SATRAK and the MATLAB outputs are insignificant. For practical uses of this data, JSC is looking at units of kilometers, which is orders of magnitudes larger than the error given by MATLAB (1.5 m maximum). However, in each case, there is an obvious periodic variation which either corresponds to the orbital period of the satellite or rotation of the Earth. This can be explained by additional types of perturbations that are modeled in SATRAK that are ignored in Vallado's code. The hypothesis is that SATRAK encompasses some form of triaxilary effects; though there is no way to test this hypothesis.

Next, the difference between the 60 minute intervals and 360 minute intervals graphs was examined to check for contradictory data (see figure 16) by overlaying data for object 8832). As expected, the two graphs show the same long periodic trend; however, the 60 minute interval captures some short-term perturbations as well. The order of magnitude difference between the two intervals is less than the initial error and can safely be 
ignored. Thus, there is no contradictory data. Similar analysis was performed on all subsequent objects and codes with similar results and therefore, are not shown.

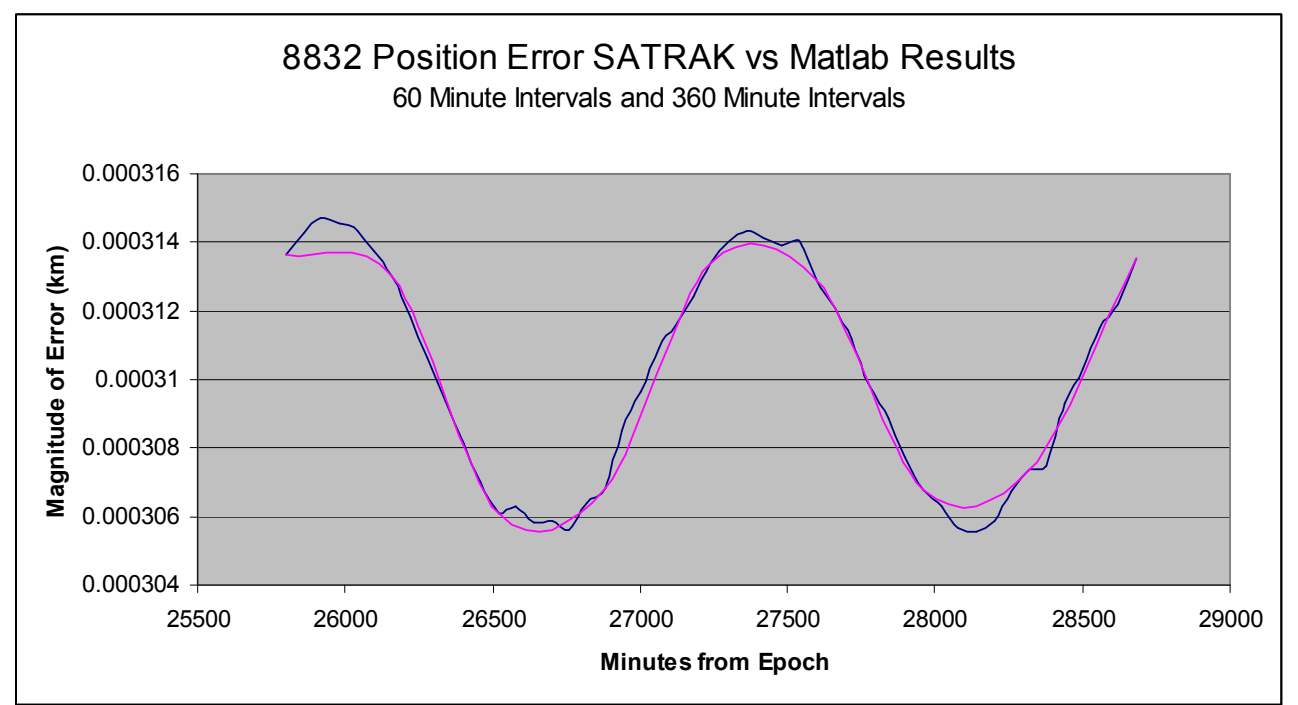

Figure 16 - Comparison of 60 and 360 minute intervals

\subsection{FORTRAN vs. SATRAK}

Results are displayed for both scenarios created in SATRAK (60 and 360 minute intervals).

\subsubsection{Object 8832}

The maximum position error calculated is $32 \mathrm{~cm}$ and the maximum velocity error calculated is $0.028 \mathrm{~mm} / \mathrm{sec}$. 


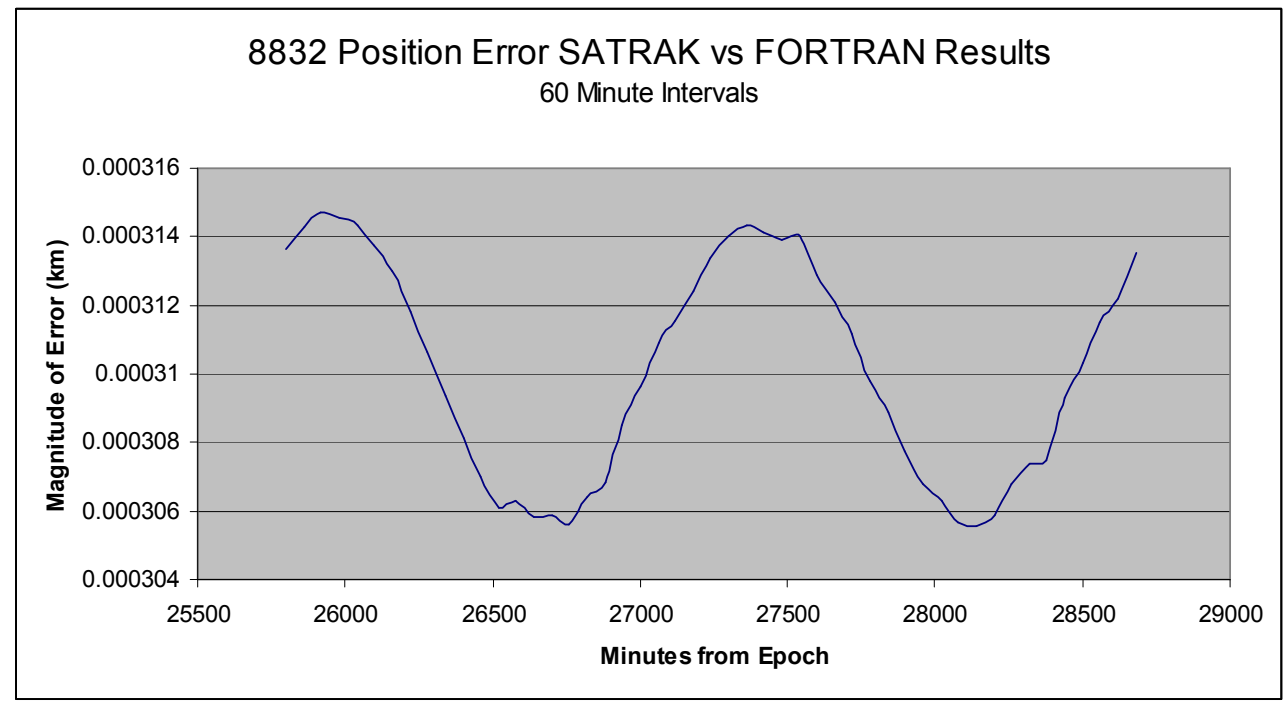

Figure 17 - 8832 Position Error FORTRAN (60 min intervals)

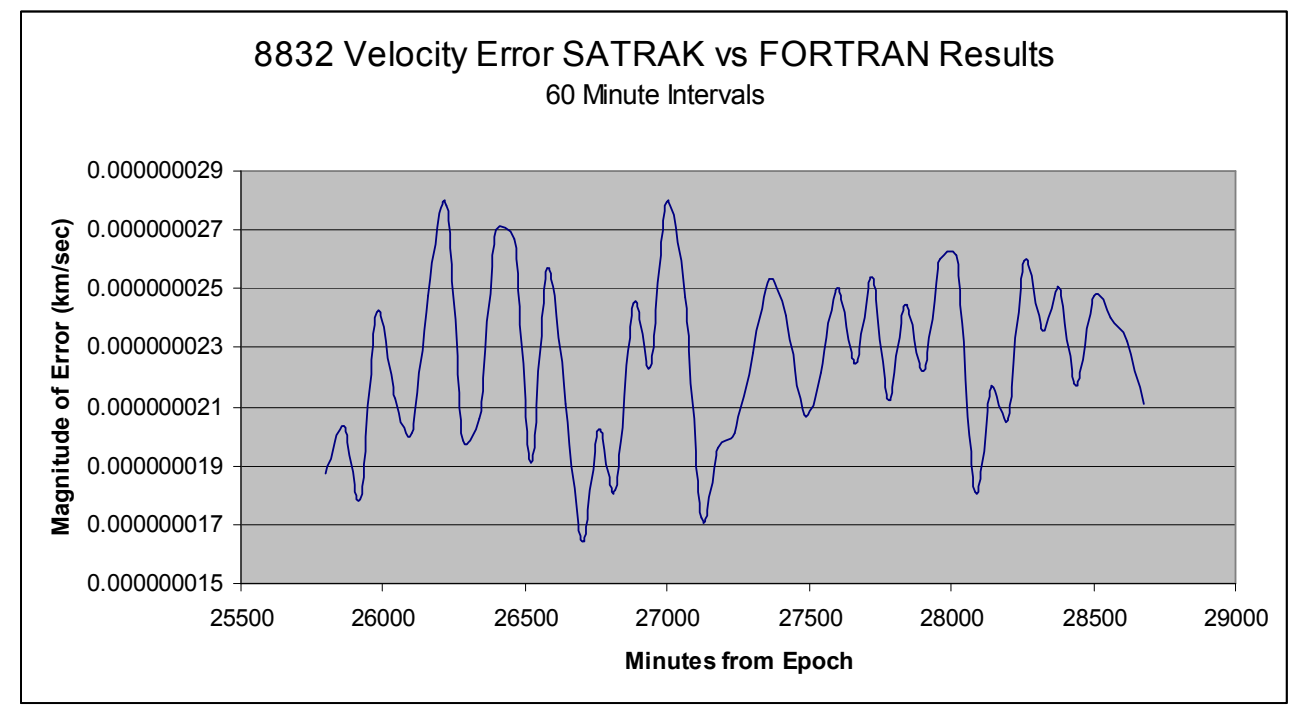

Figure 18 - 8832 Velocity Error FORTRAN (60 min intervals) 


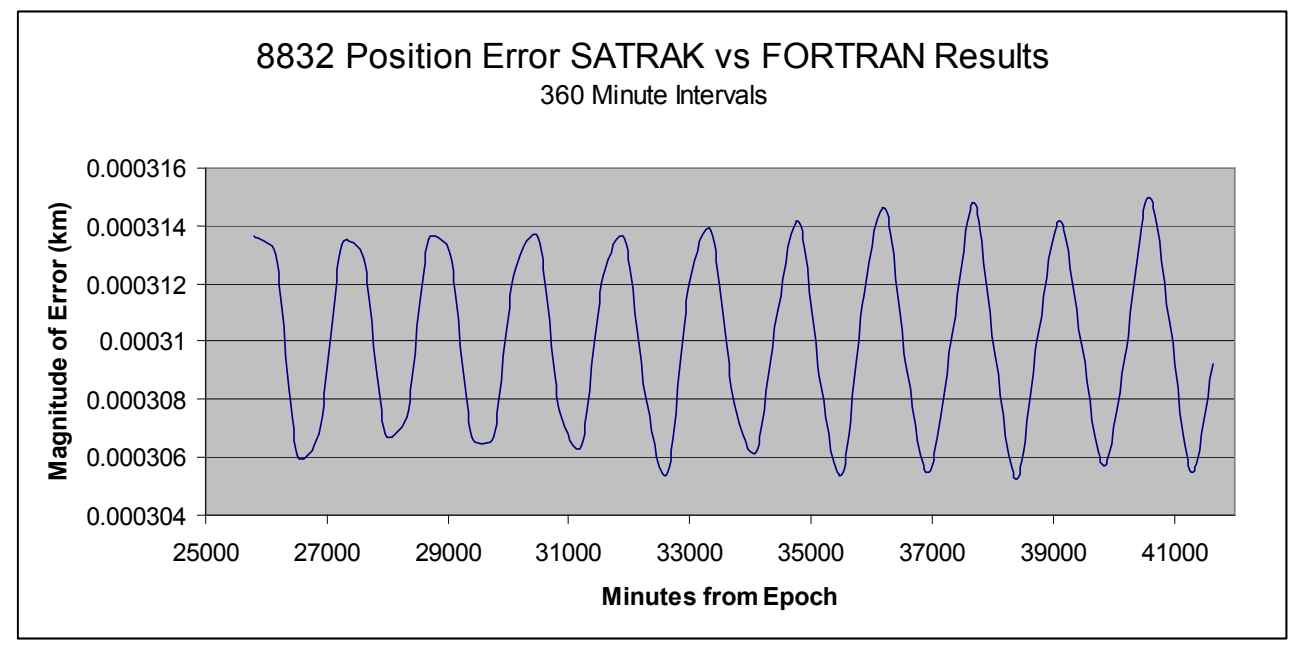

Figure 19 - 8832 Position Error FORTRAN (360 min intervals)

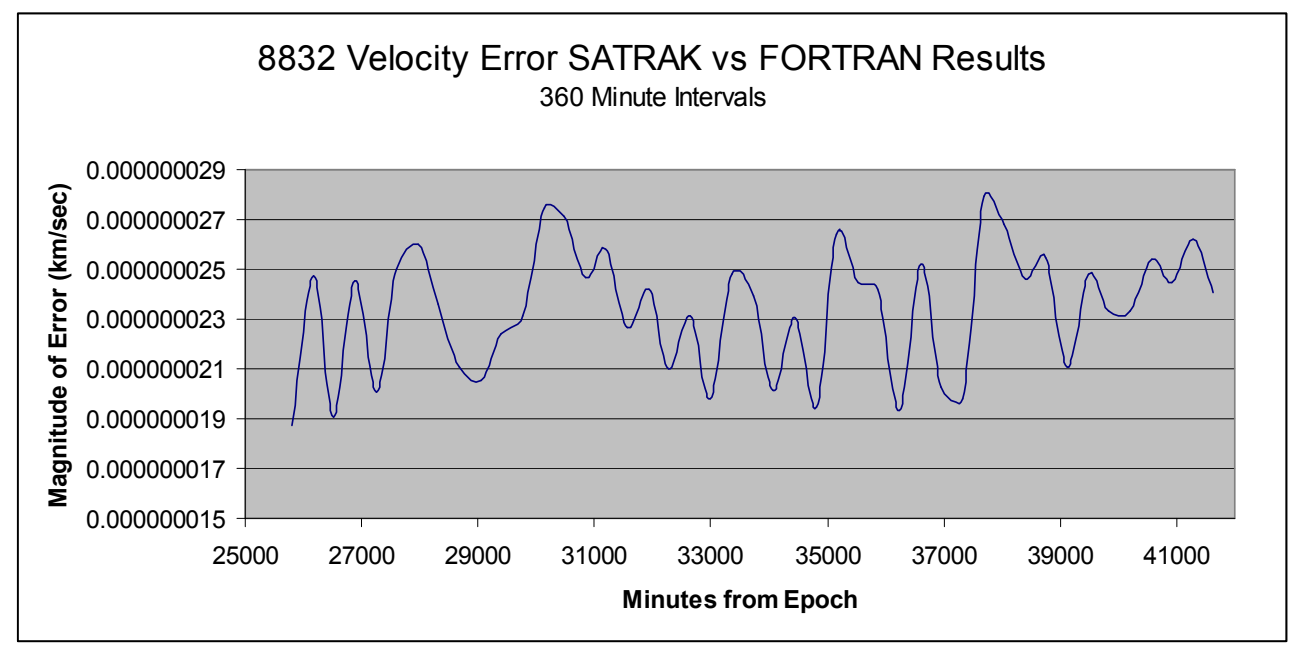

Figure 20 - 8832 Velocity Error FORTRAN (360 min intervals)

\subsubsection{Object 25000}

The maximum position error calculated is $1.5 \mathrm{~m}$ and the maximum velocity error calculated is $0.11 \mathrm{~mm} / \mathrm{sec}$ 


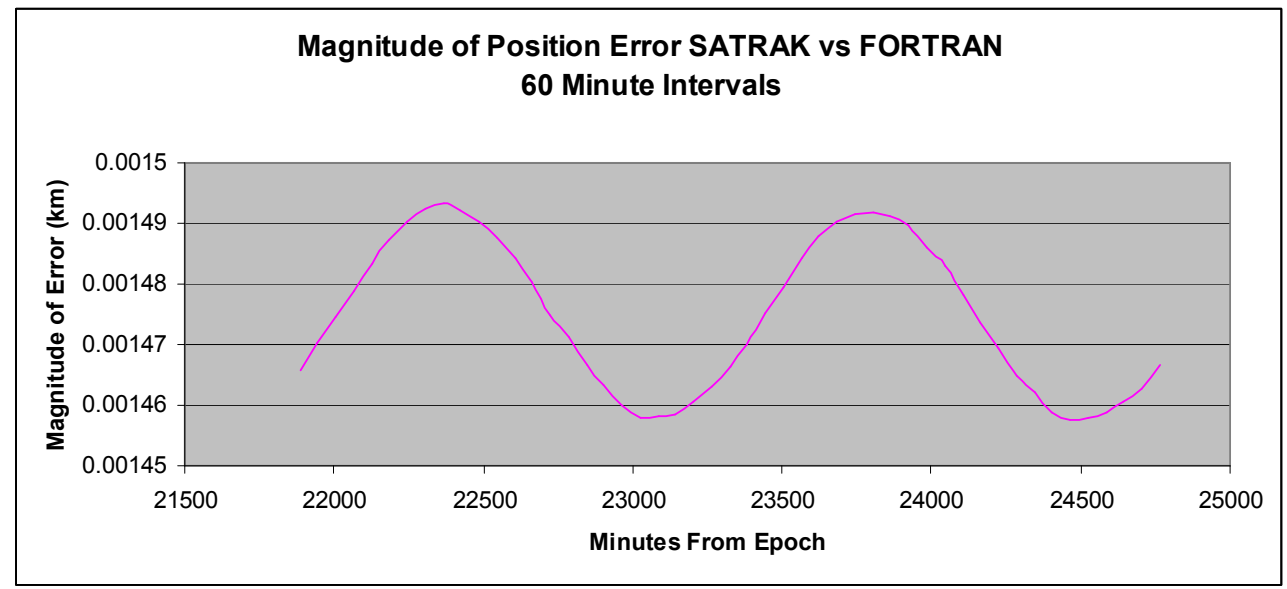

Figure 21 - 25000 Position Error FORTRAN (60 min intervals)

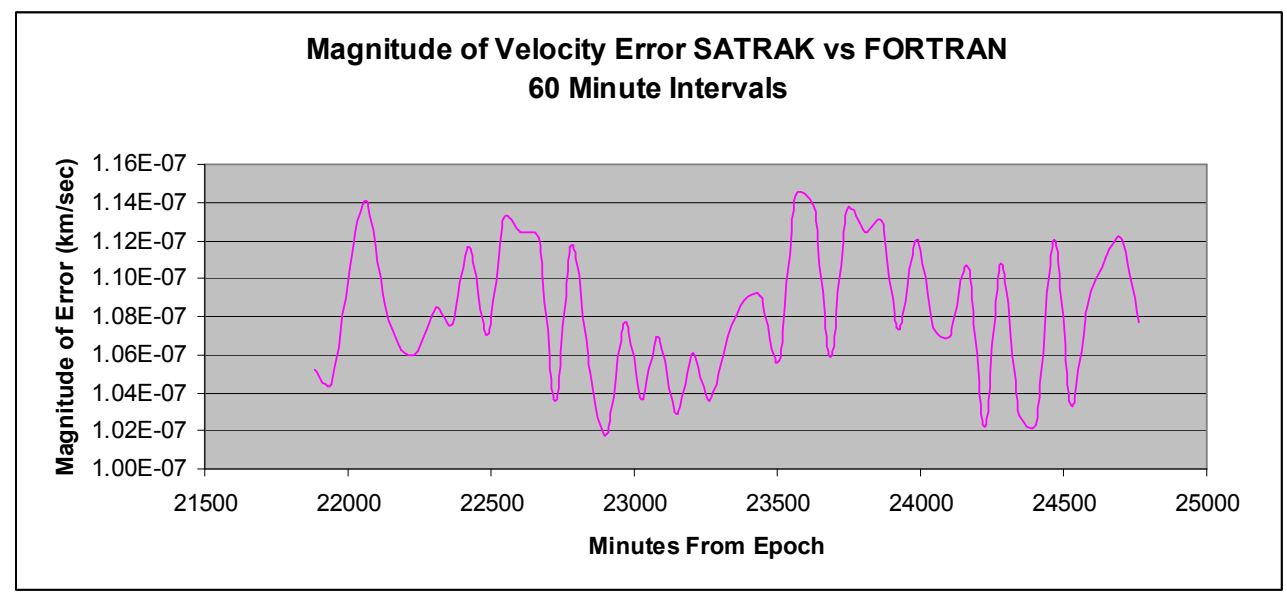

Figure 22 - 25000 Velocity Error FORTRAN (60 min intervals) 


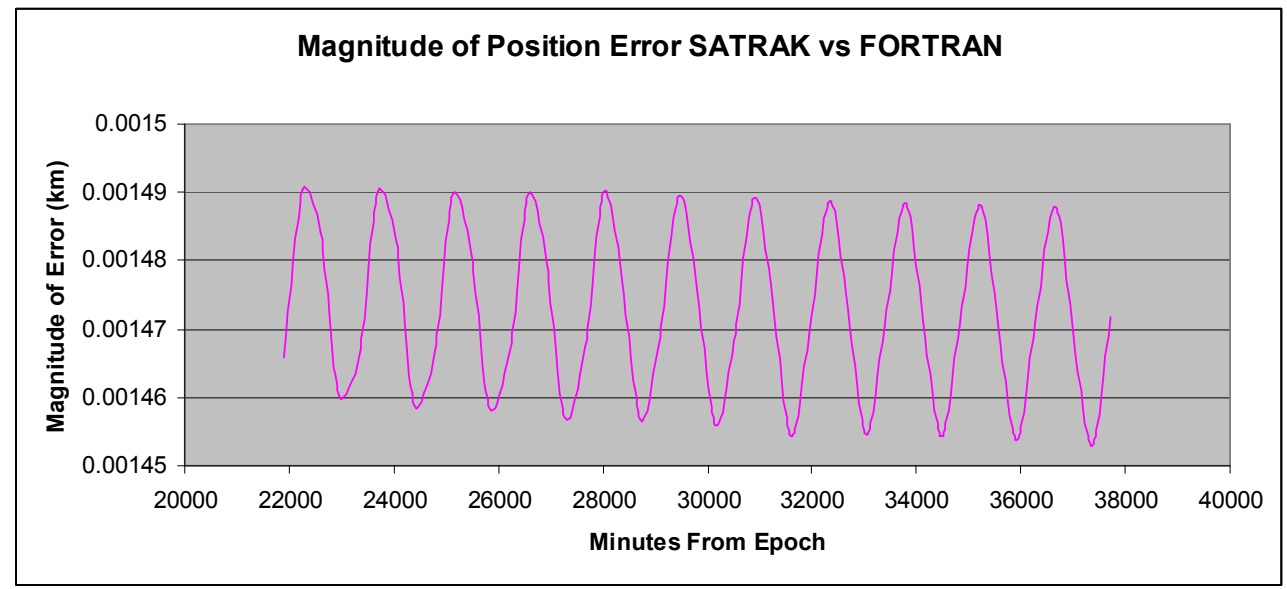

Figure 23 - 25000 Position Error FORTRAN (360 min intervals)

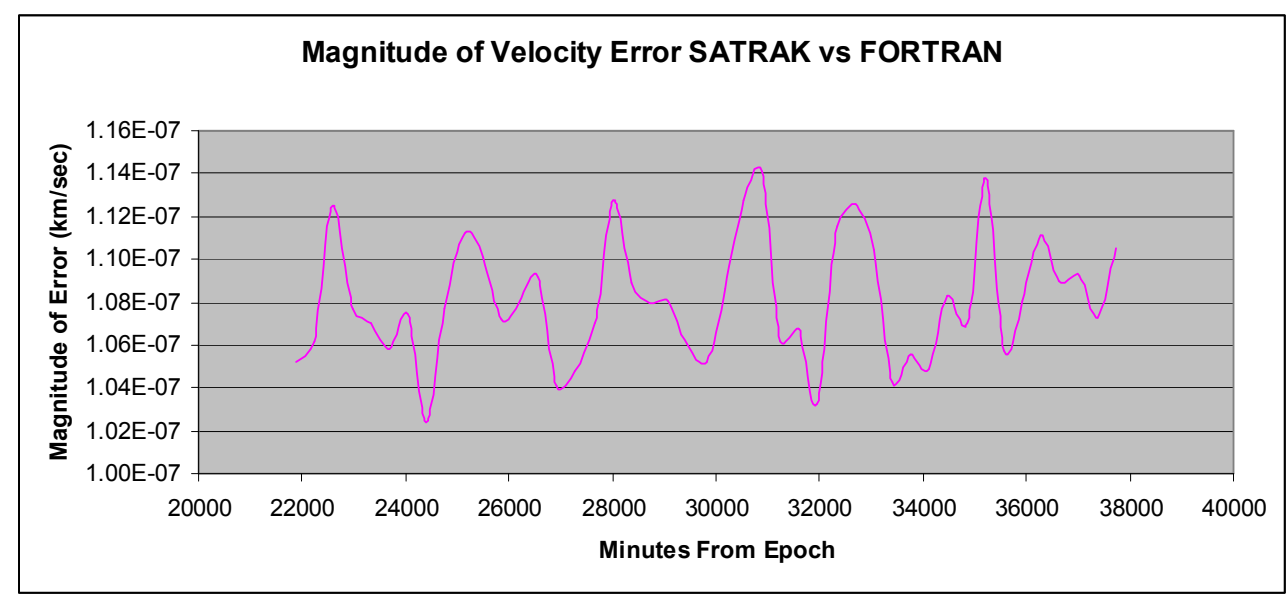

Figure 24 - 25000 Velocity Error FORTRAN (60 min intervals)

\subsubsection{Object 30000}

The maximum position error between the FORTRAN and SATRAK results is $53 \mathrm{~cm}$ and the maximum velocity error is $4.4 \mathrm{~mm} / \mathrm{sec}$. 


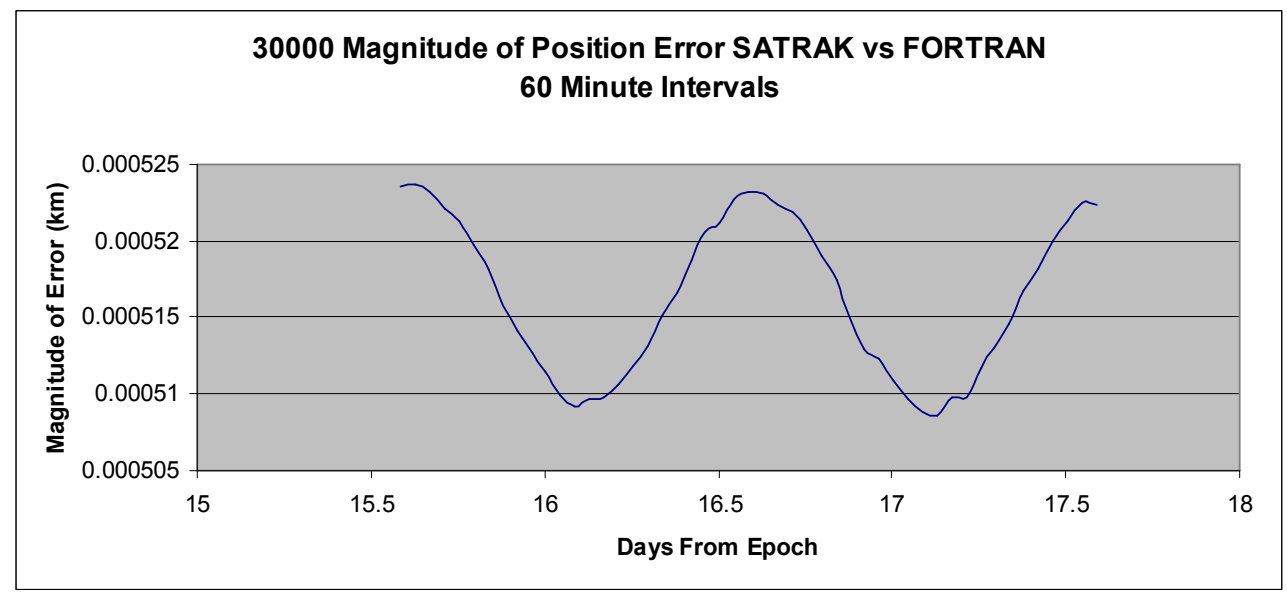

Figure 25 - 30000 Position Error FORTRAN (60 min intervals)

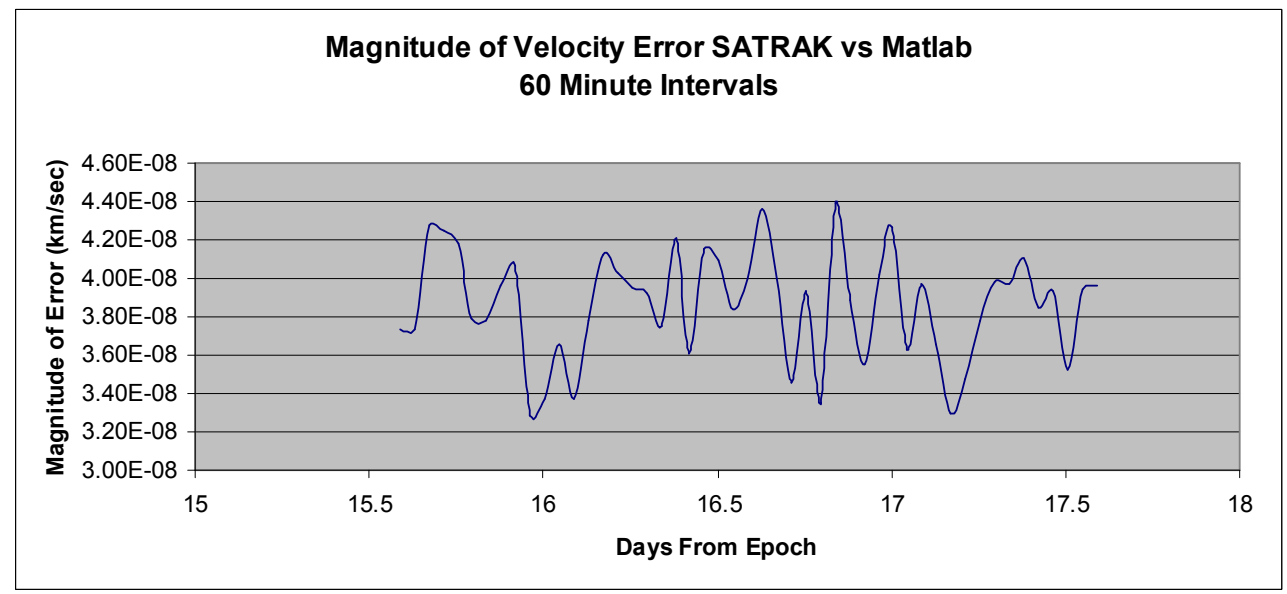

Figure 26 - 30000 Velocity Error FORTRAN (60 min intervals) 


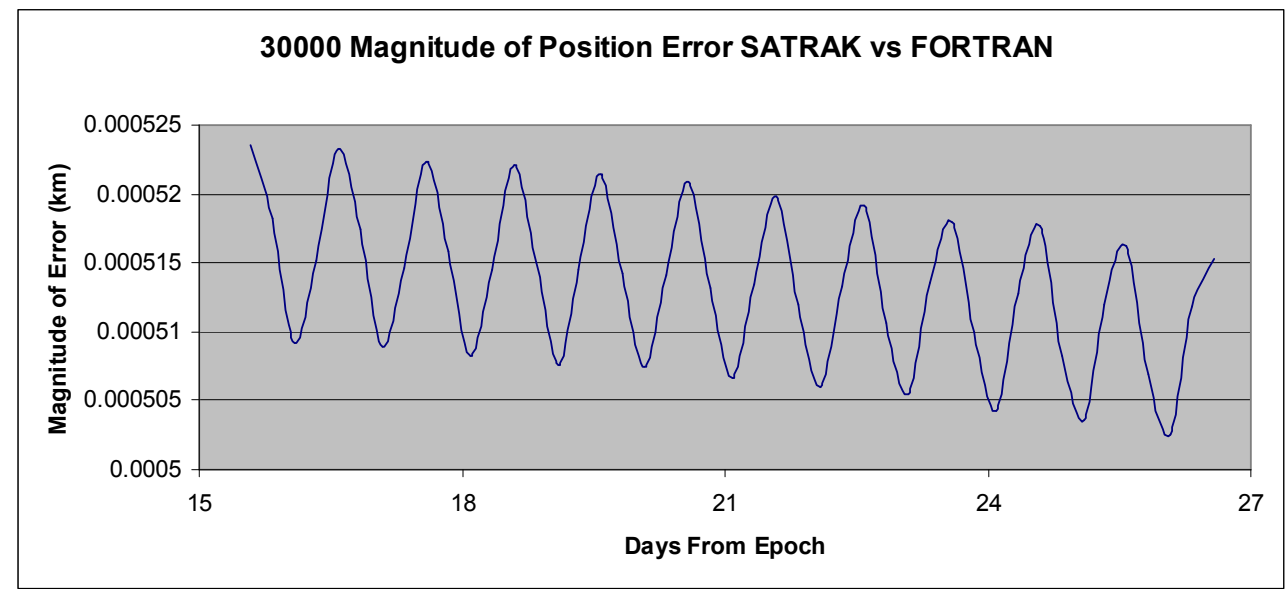

Figure 27 - 30000 Position Error FORTRAN (360 min intervals)

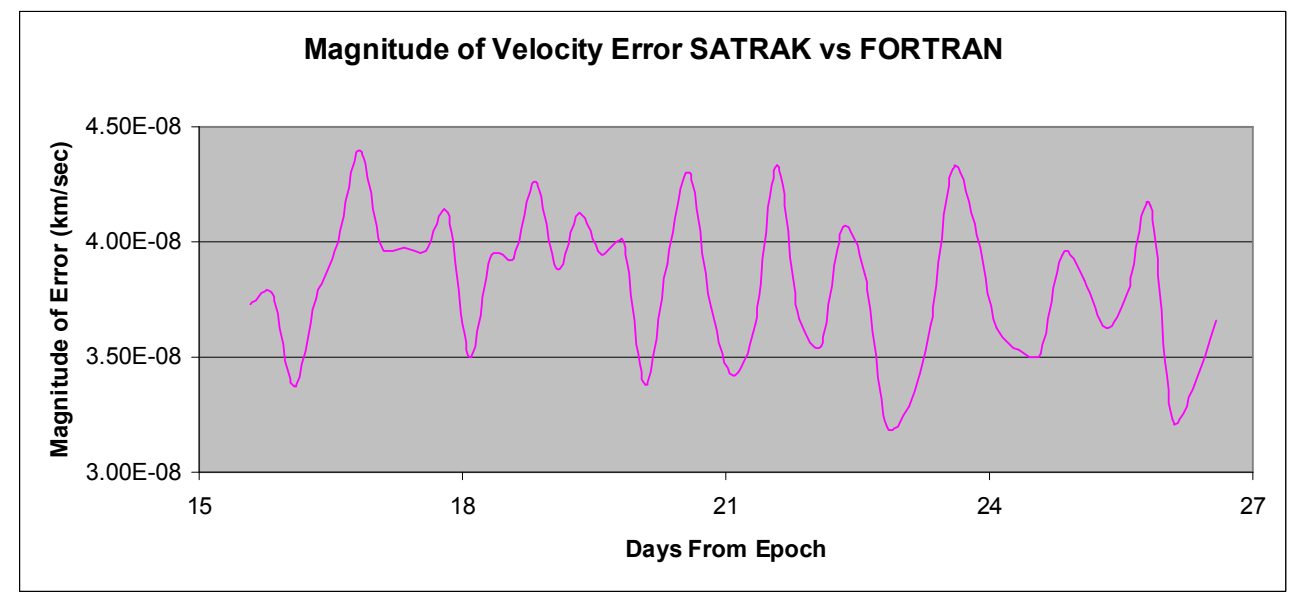

Figure 28 - 30000 Velocity Error FORTRAN (360 min intervals)

\subsubsection{Analysis - FORTRAN}

The FORTRAN code outputs very similar data to the MATLAB code in terms of position (see below). From visual inspection the two sets of data are indistinguishable. 


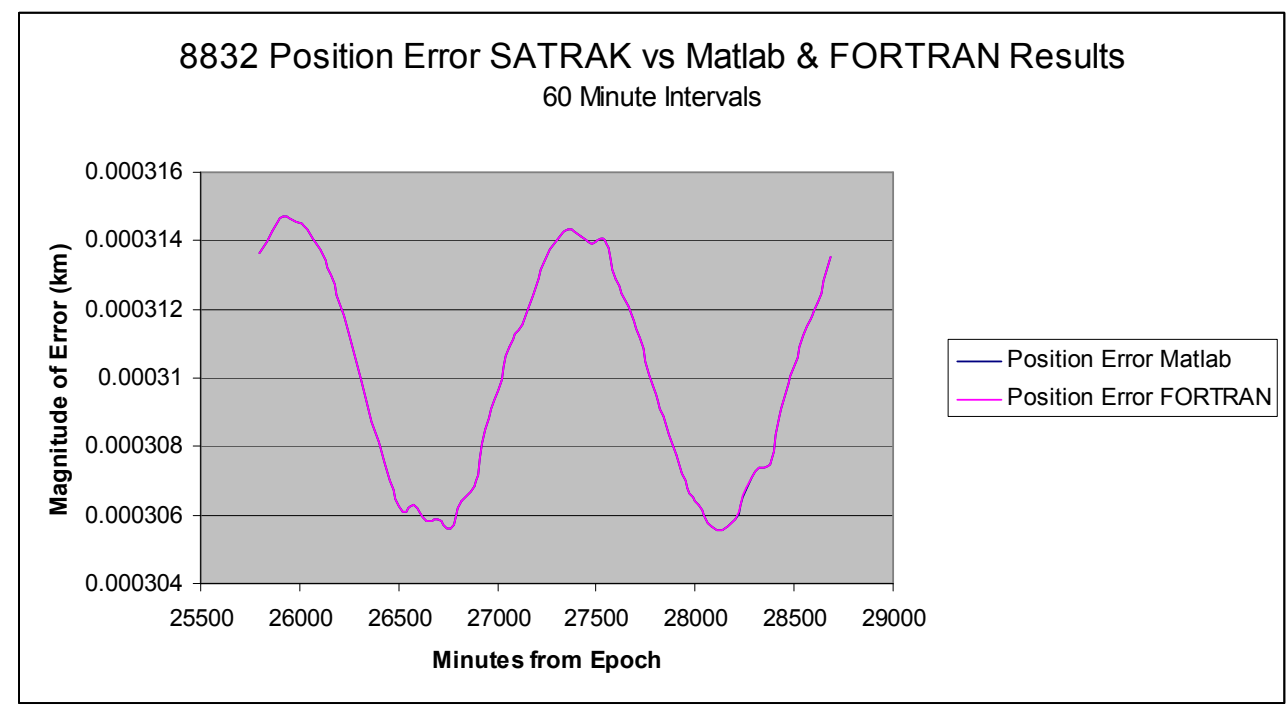

Figure 29 - Comparison of MATLAB and FORTRAN results (8832 position)

There is slightly more difference in the velocity output, see figure 26 (object 8832). The cause of the variation in the output of the FORTRAN code is unknown, but consistent with results found with object 25000. An initial hypothesis was a difference in the precision of the numbers carried through the FORTRAN calculations versus the MATLAB calculations; however, further results from the next section show otherwise. Though the fluctuations look significant, please remember that the graph is displaying differences of $.004 \mathrm{~mm} / \mathrm{sec}$ between the output of the data which is describing the motion of an object traveling roughly $3 \mathrm{~km} / \mathrm{sec}$. 


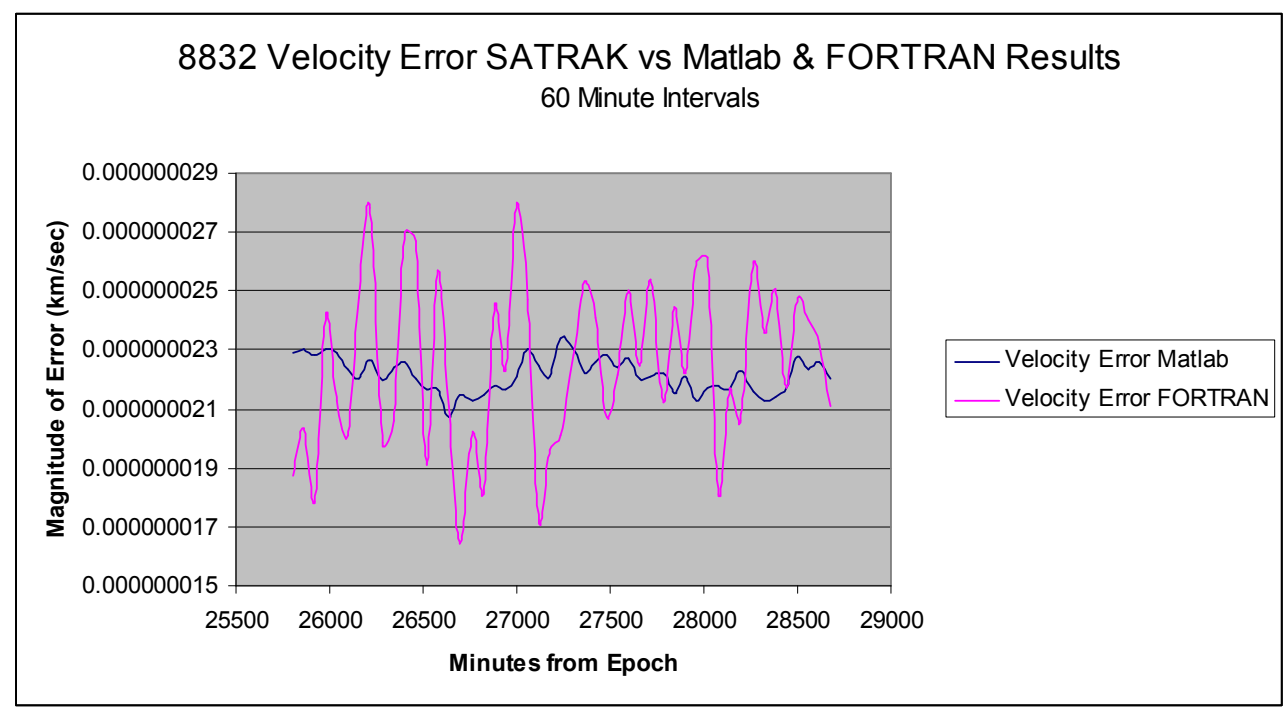

Figure 30 - Comparison of MATLAB and FORTRAN (8832 Velocity)

\subsection{Modified Code vs. SATRAK}

Results are displayed for both scenarios created in SATRAK (60 and 360 minute intervals). The modified code (labeled Vallado in the graphs) is designed to simulate the inputs and outputs of NASA JSC's existing code. Due to increased complexity, epoch data is also included as an input to the system. This leads to further complications when installing the upgrade into the PREDICT code, which is discussed in a later section.

\subsubsection{Object 8832}

The maximum position error calculated is $32 \mathrm{~cm}$ and the maximum velocity error calculated is $0.024 \mathrm{~mm} / \mathrm{sec}$. 


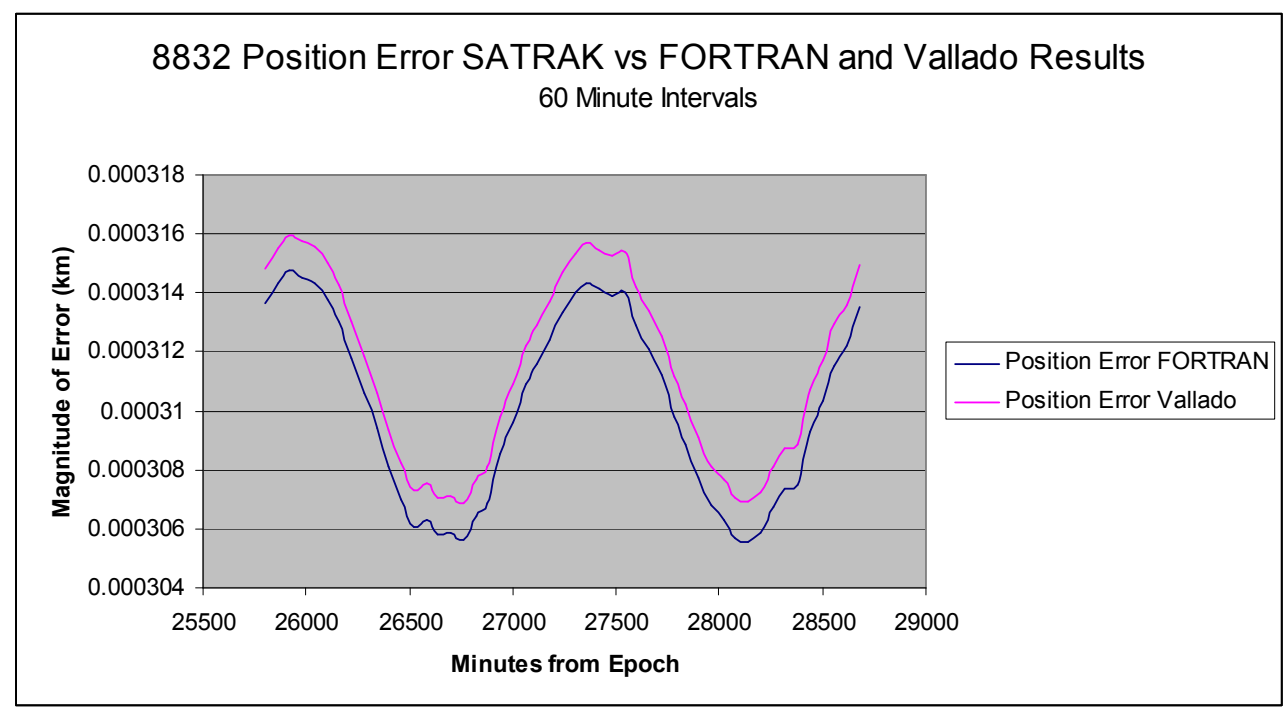

Figure 31 - 8832 Position Error Vallado (60 min intervals)

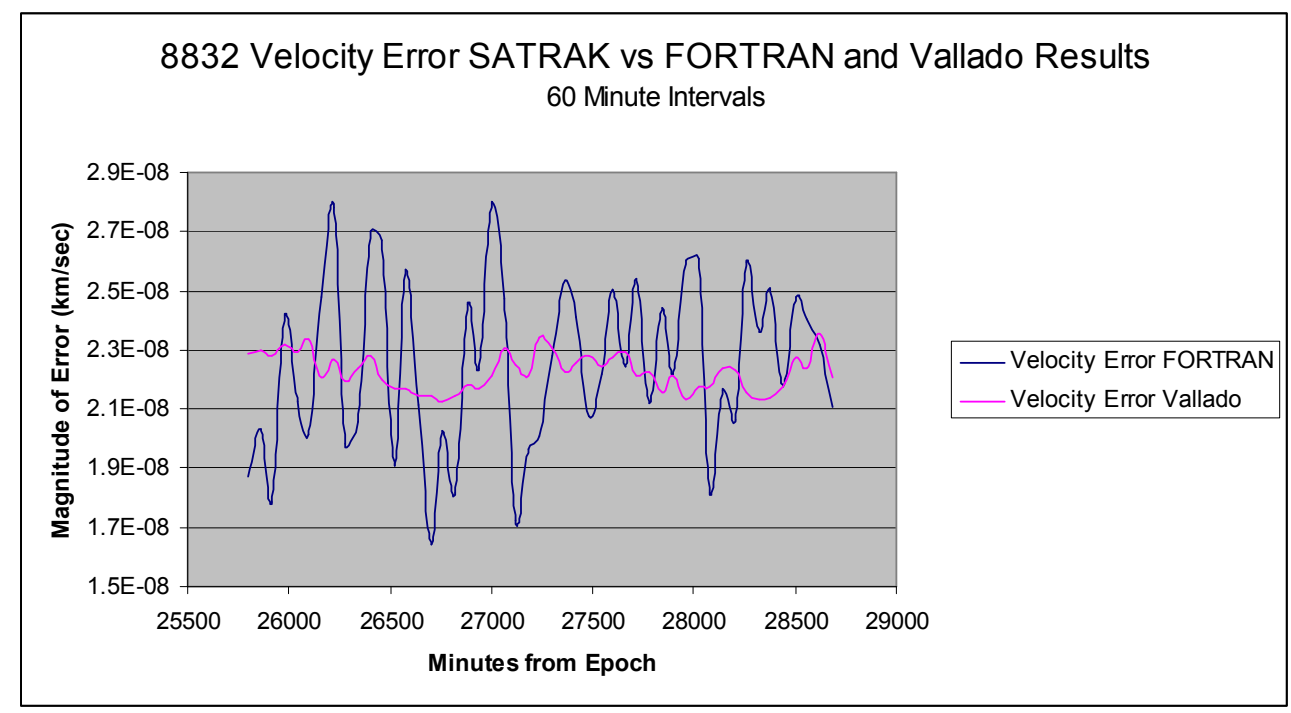

Figure 32 - 8832 Velocity Error Vallado (60 min intervals) 


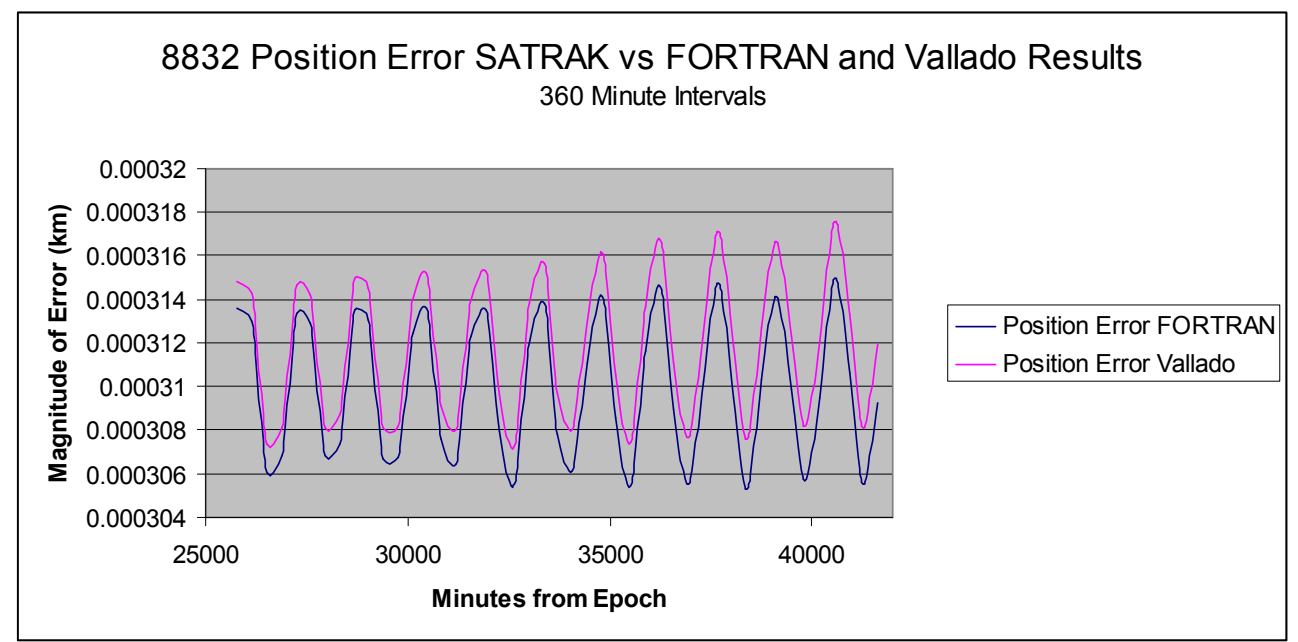

Figure 33 - 8832 Position Error Vallado (360 min intervals)

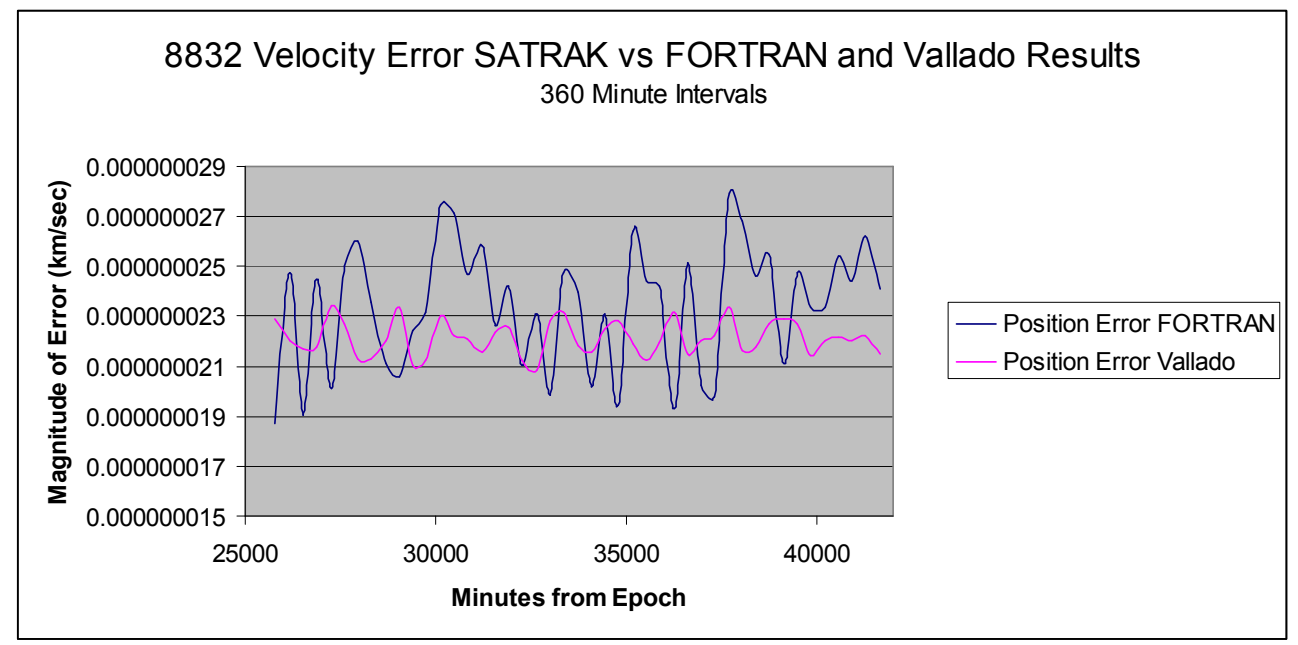

Figure 34 - 8832 Velocity Error Vallado (360 min intervals)

\subsubsection{Object 25000}

The maximum position error calculated is $1.5 \mathrm{~m}$ and the maximum velocity error calculated is $.11 \mathrm{~mm} / \mathrm{sec}$. 


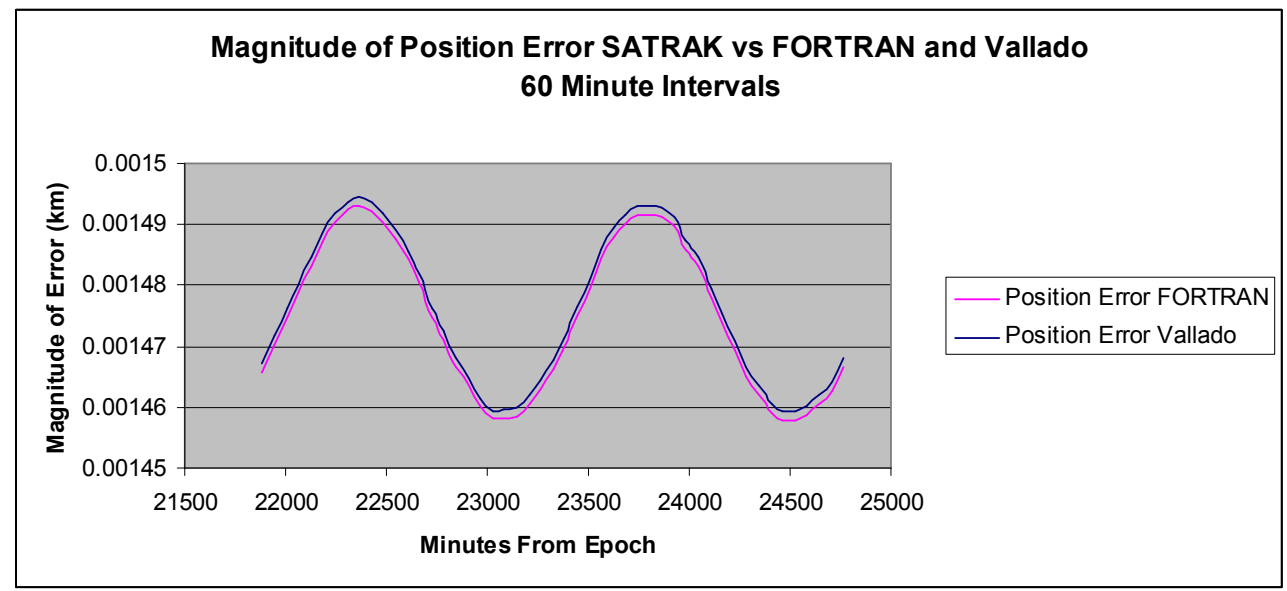

Figure 35 - 25000 Position Error Vallado (60 min intervals)

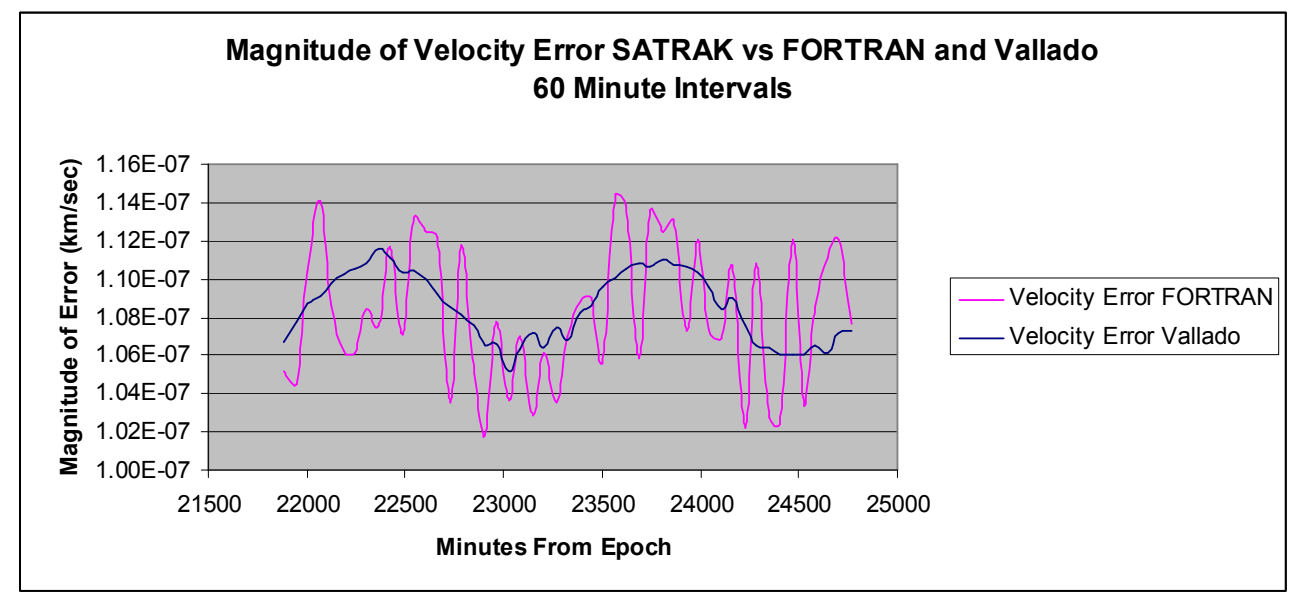

Figure 36 - 25000 Velocity Error Vallado (60 min intervals) 


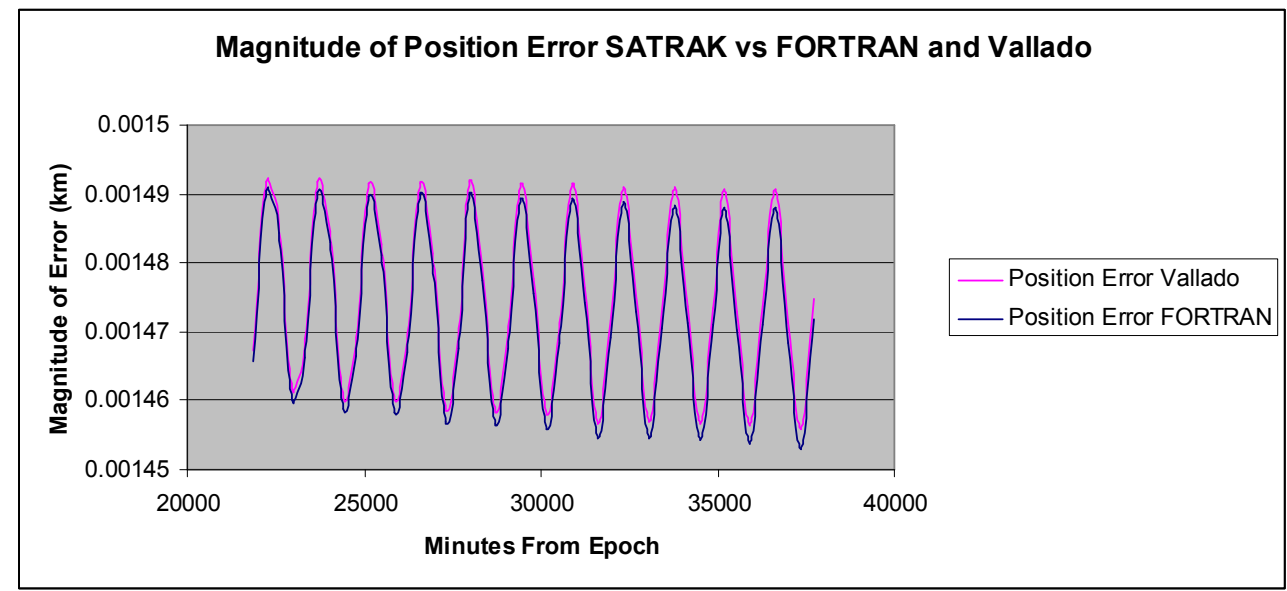

Figure 37 - 25000 Position Error Vallado (360 min intervals)

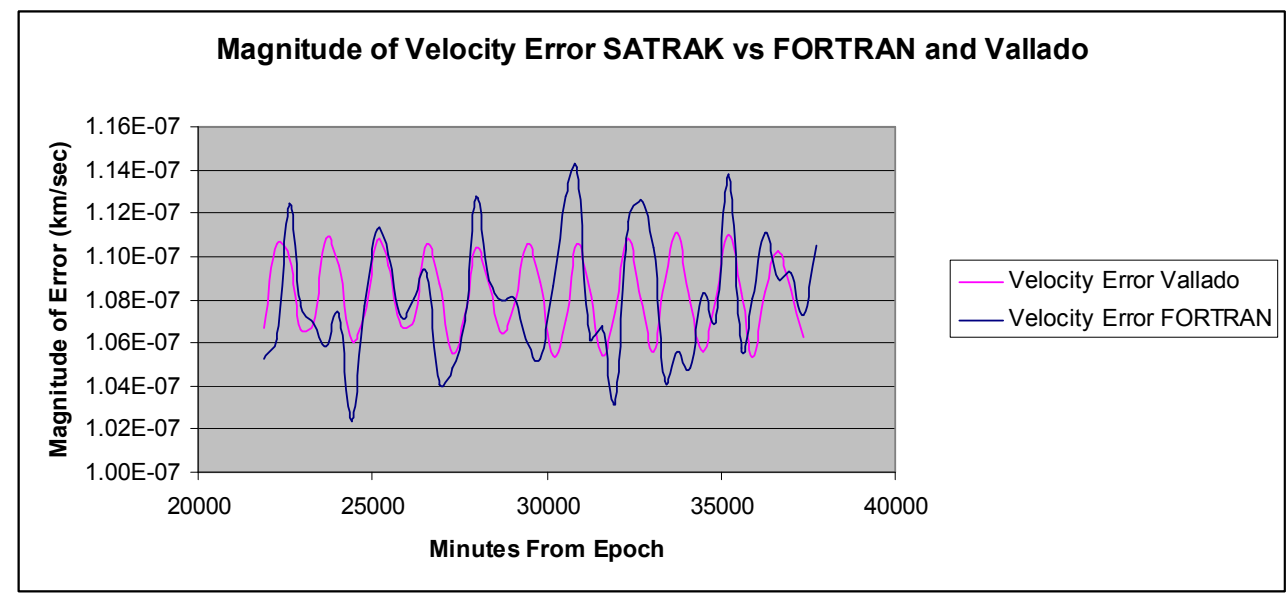

Figure 38 - 25000 Velocity Error Vallado (360 min intervals)

\subsubsection{Object 30000}

The maximum position error calculated is $6.3 \mathrm{~m}$ and the maximum velocity error calculated is $0.47 \mathrm{~mm} / \mathrm{sec}$. 


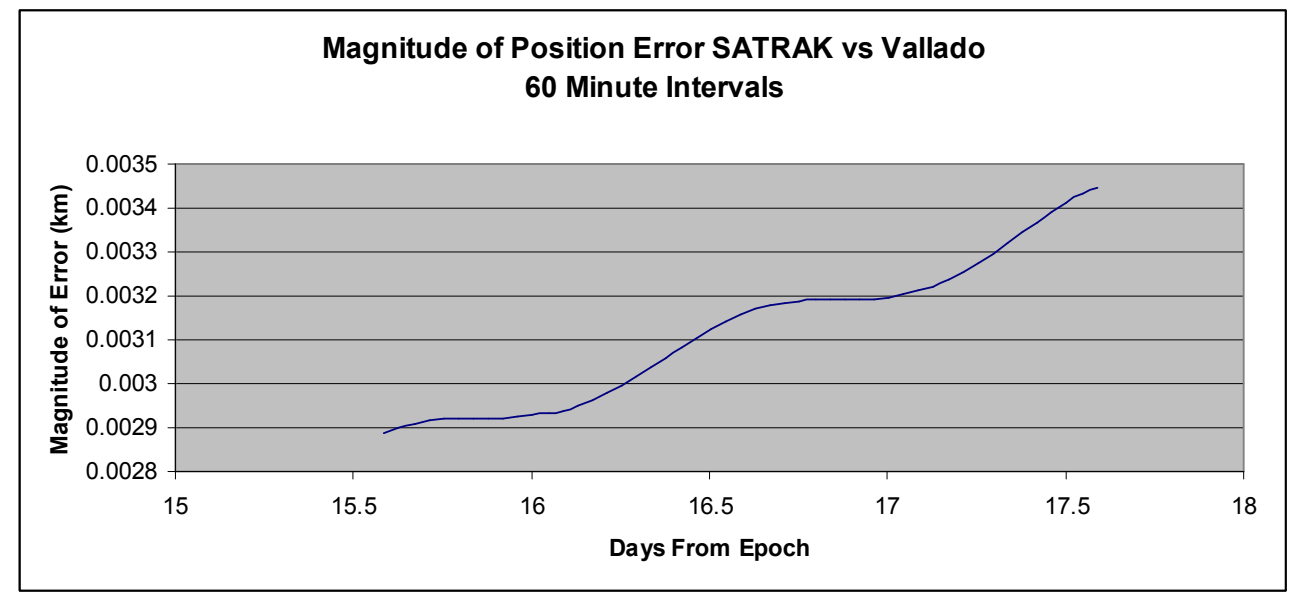

Figure 39 - 30000 Position Error Vallado (60 min intervals)

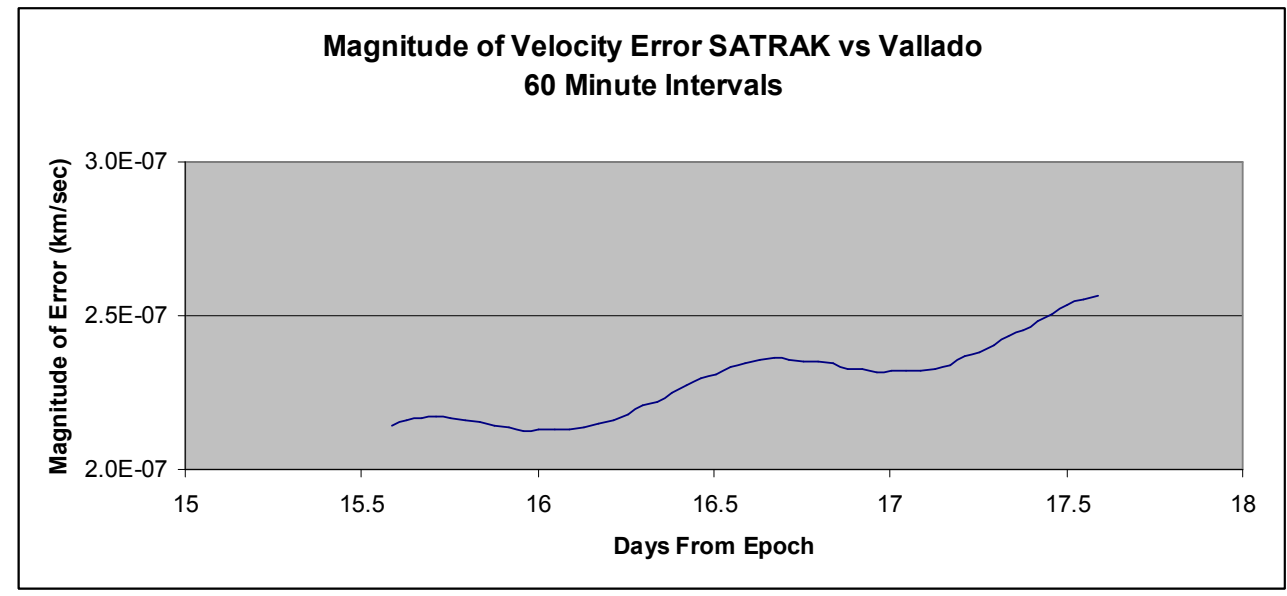

Figure 40 - 30000 Velocity Error Vallado (60 min intervals) 


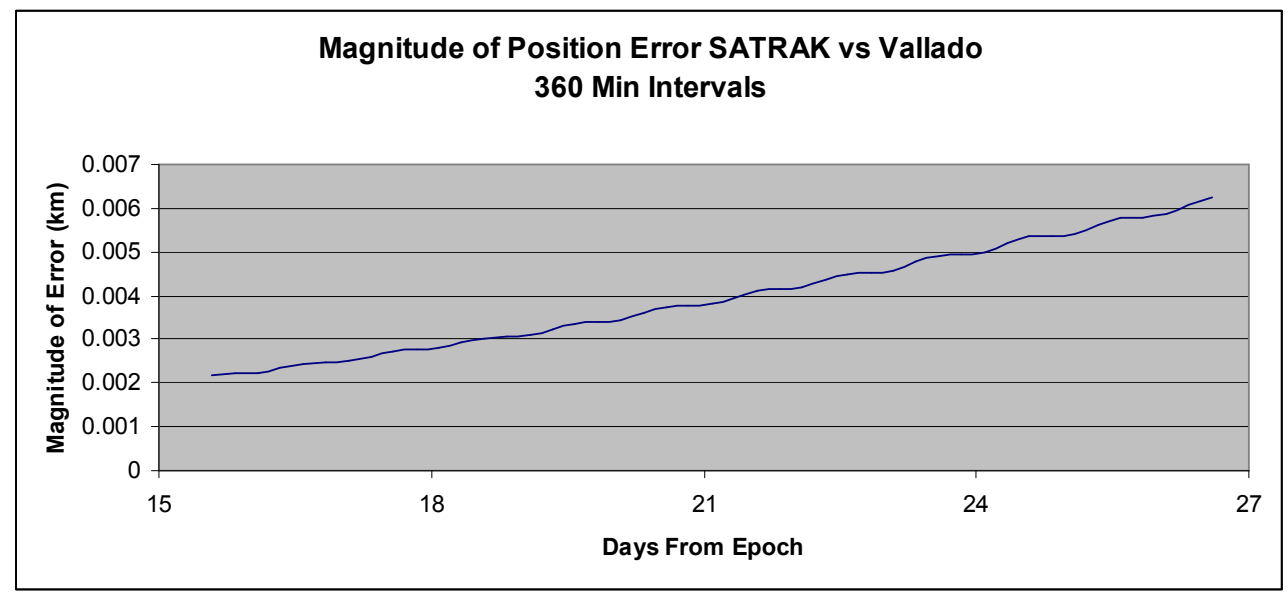

Figure 41 - 30000 Position Error Vallado (360 min intervals)

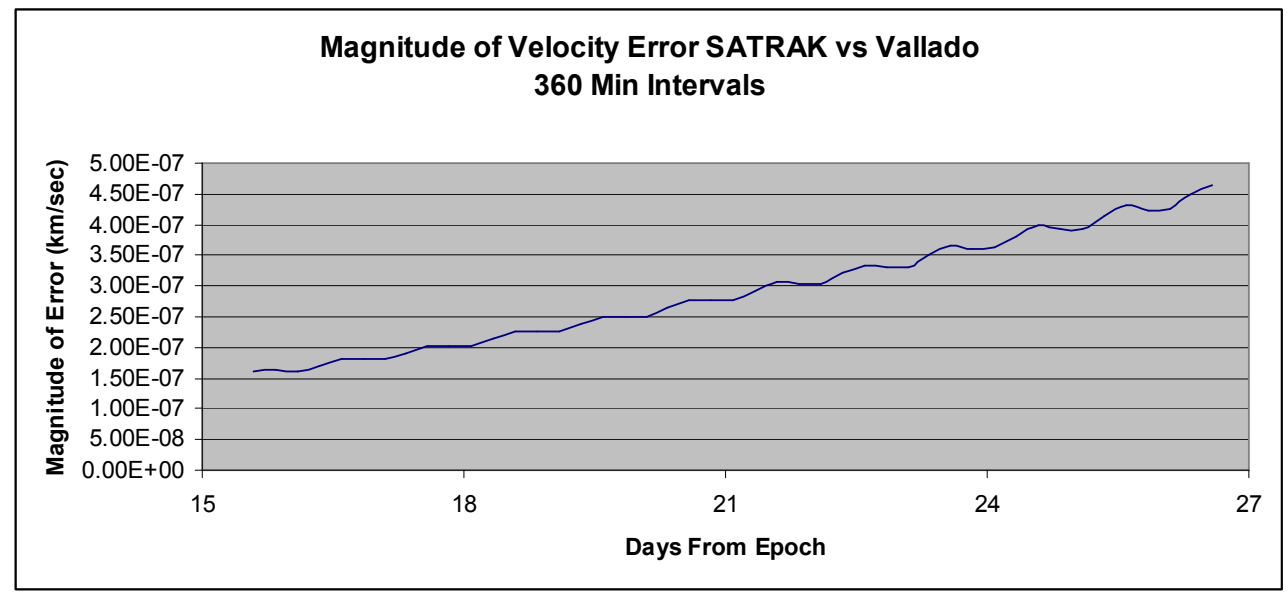

Figure 42 - 30000 Velocity Error Vallado (360 min intervals)

\subsubsection{Analysis - Modified Code}

Since the Vallado code is a stripped down version of the FORTRAN code, it is expected that the results come out equal or close to equal. Looking at the resultant graphs for object 8832 and object 25000 show some noteworthy trends. First, that the position error 
for the full FORTRAN code provides results slightly closer to truth. In the two cases, FORTRAN code is consistently fractions of a millimeter better. Why this happens is unknown. Second, the velocity error is more similar to the MATLAB error than the FORTRAN error. The reason for this is also unknown, but can be seen in figure 39 .

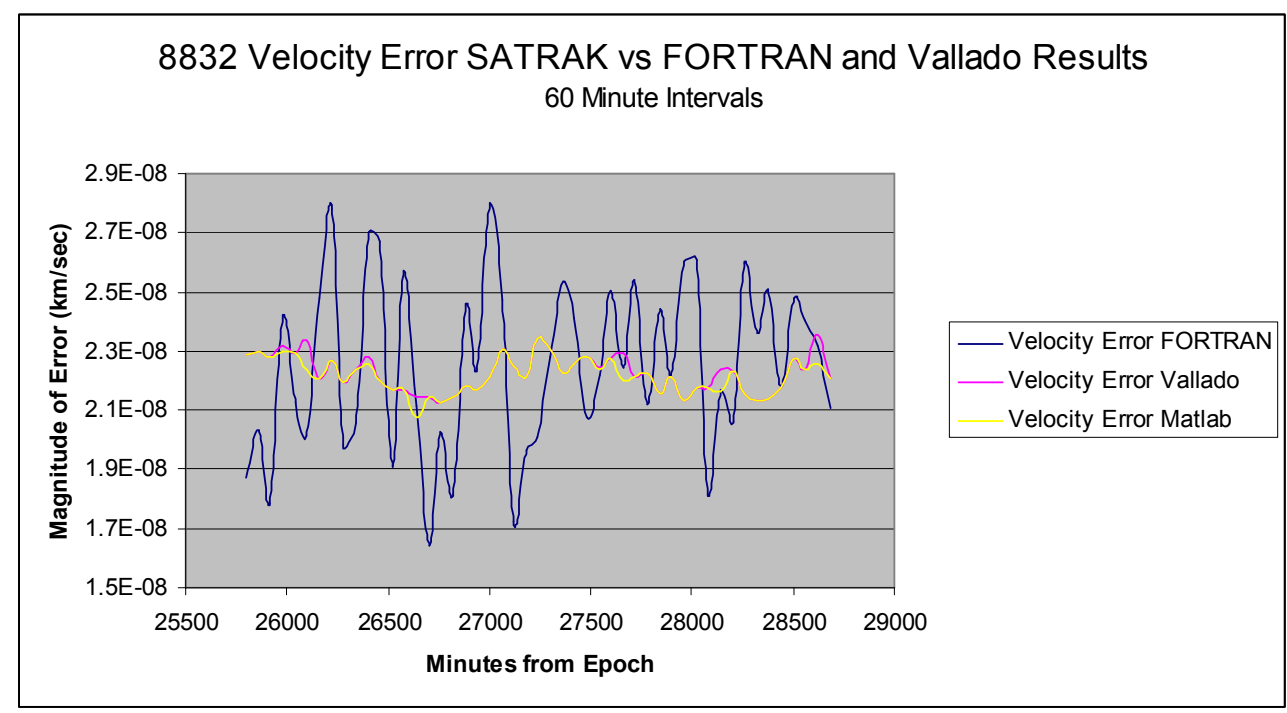

Figure 43 - Comparison of Vallado to MATLAB (8832 Velocity Error)

Finally, the results of object 30000 do not match the pattern initiated by the first two objects. The error involved is much greater than other runs and though has periodic tendencies, adheres more towards linear growth. Even though the error is larger than other runs, it is still orders of magnitude less than the operating threshold.

\subsection{JSC Code vs. SATRAK}

The JSC SGP4 subroutine code was taken directly from October 2008 version of the PREDICT code. In these tests, it is called by the same program that calls the modified code. In the resultant graphs, the JSC code is labeled as Matney, after Dr. Matney who wrote the original code. 


\subsubsection{Object 8832}

The maximum position error calculated is $356.1 \mathrm{~km}$ and the maximum velocity error calculated is $25.4 \mathrm{~m} / \mathrm{sec}$.

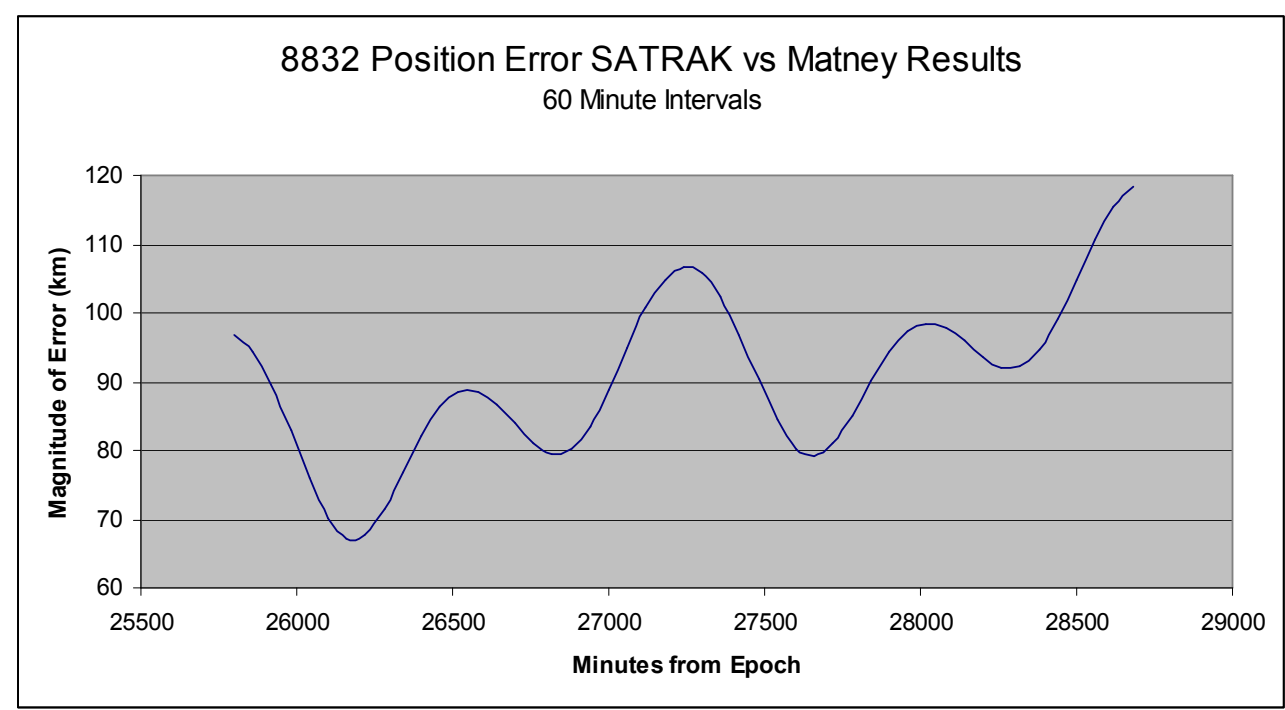

Figure 44 - 8832 Position Error Matney (60 min intervals) 


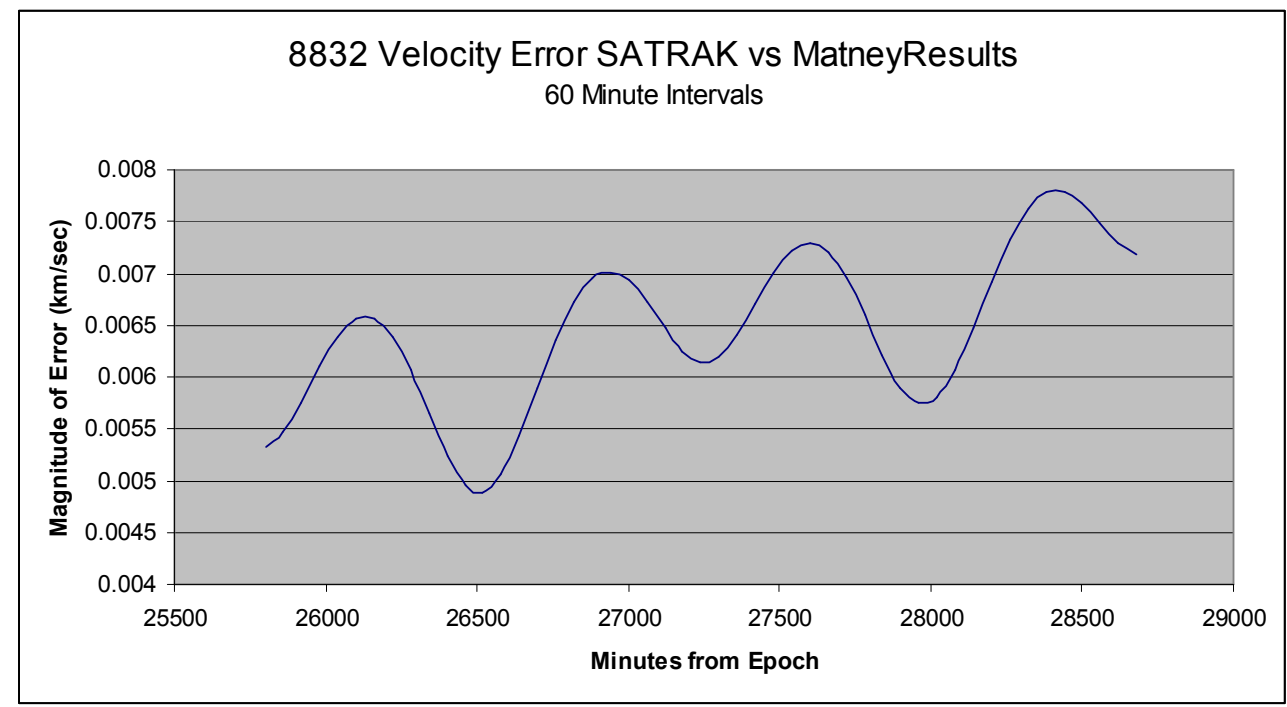

Figure 45 - 8832 Velocity Error Matney (60 min intervals)

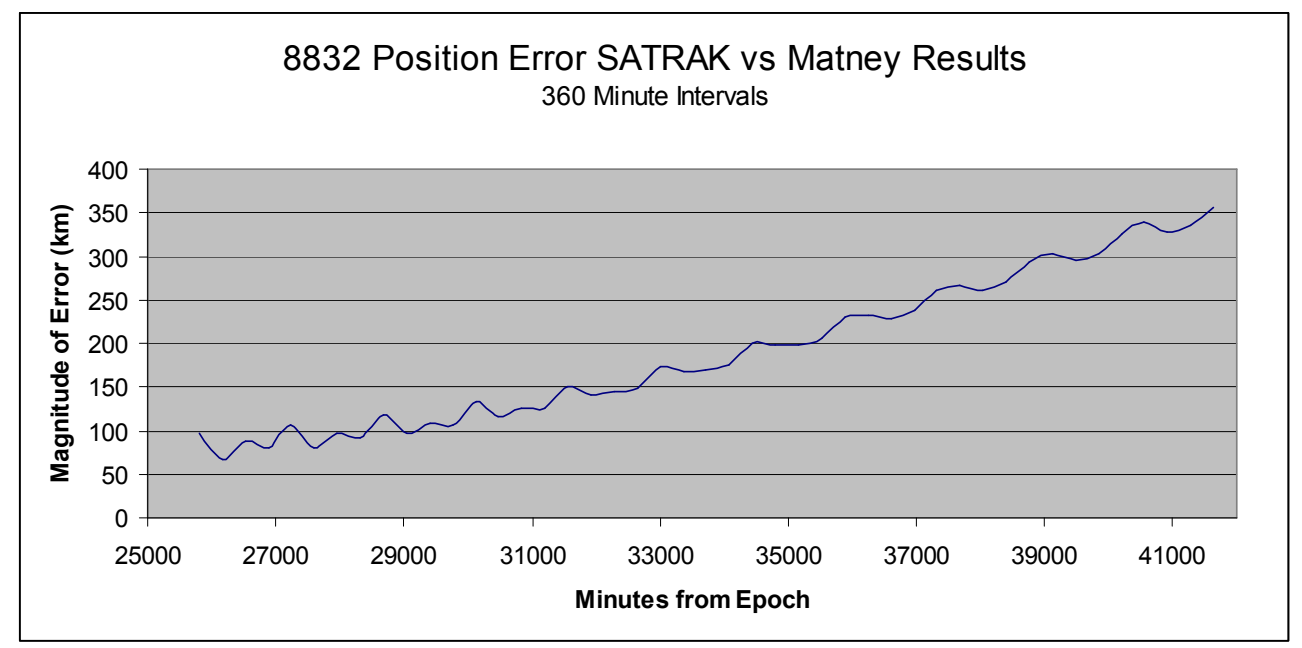

Figure 46 - 8832 Position Error Matney (360 min intervals) 


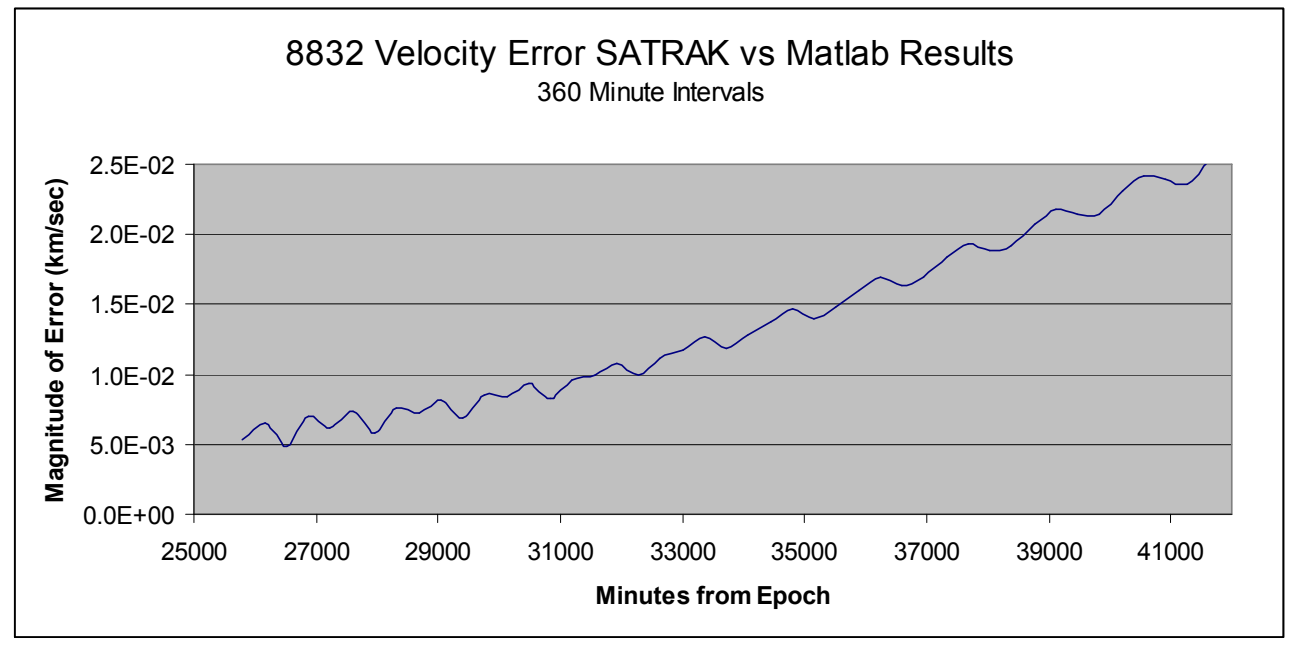

Figure 47 - 8832 Velocity Error Matney (360 min intervals)

\subsubsection{Object 25000}

The maximum position error calculated is $137.8 \mathrm{~km}$ and the maximum velocity error calculated is $10 \mathrm{~m} / \mathrm{sec}$.

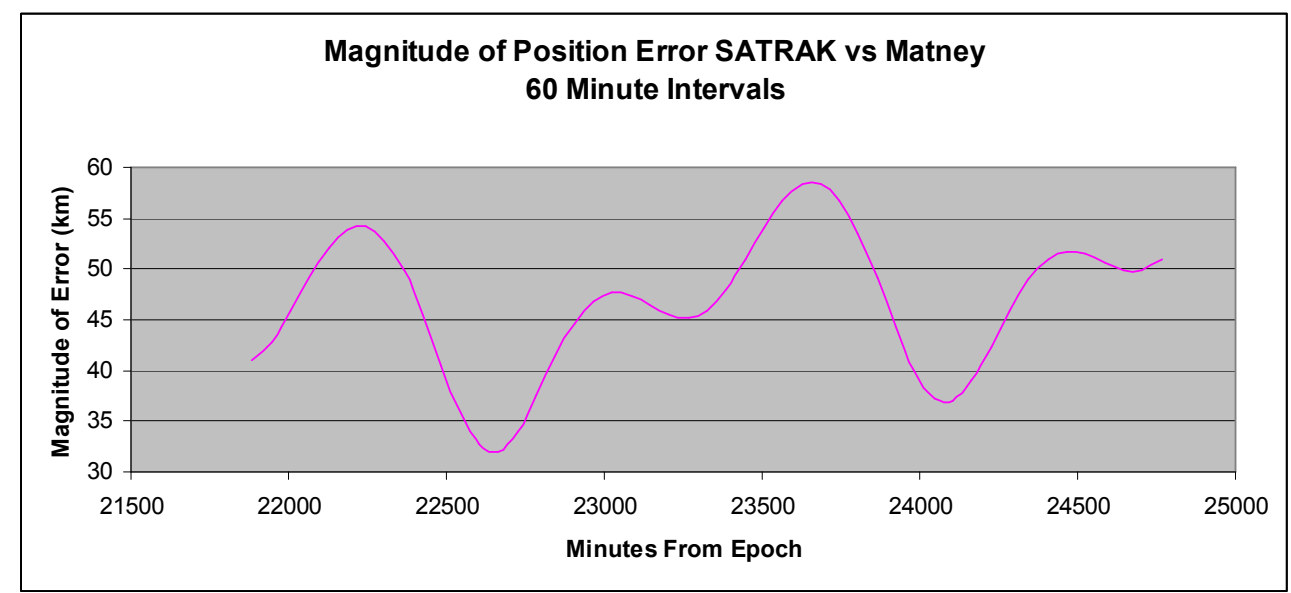

Figure 48 - 25000 Position Error Matney (60 min intervals) 


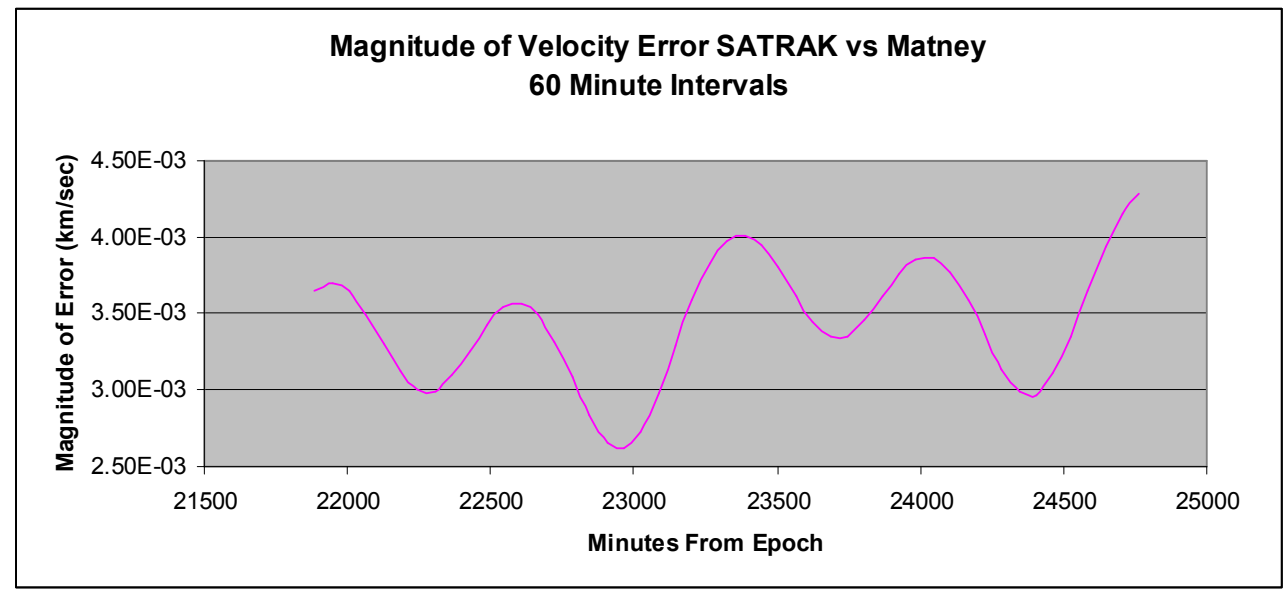

Figure 49 - 25000 Velocity Error Matney (60 min intervals)

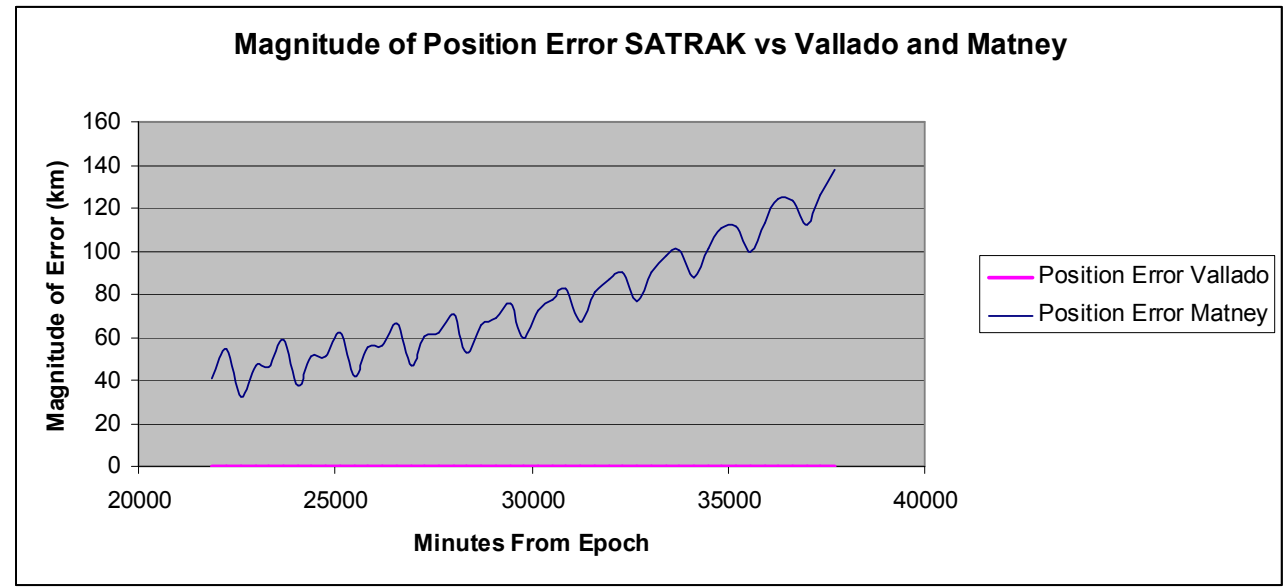

Figure 50 - 25000 Position Error Matney (360 min intervals) 


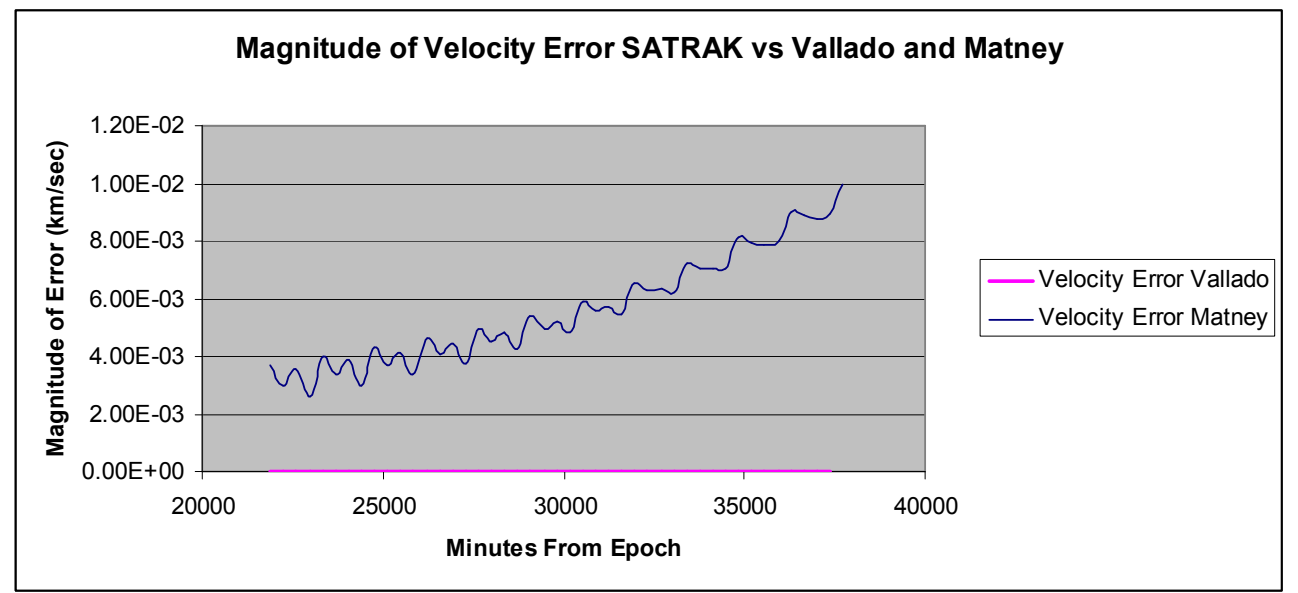

Figure 51 - 25000 Velocity Error Matney (360 min intervals) 


\subsubsection{Object 30000}

The maximum position error calculated is $61.2 \mathrm{~km}$ and the maximum velocity error calculated is $4.4 \mathrm{~m} / \mathrm{sec}$.

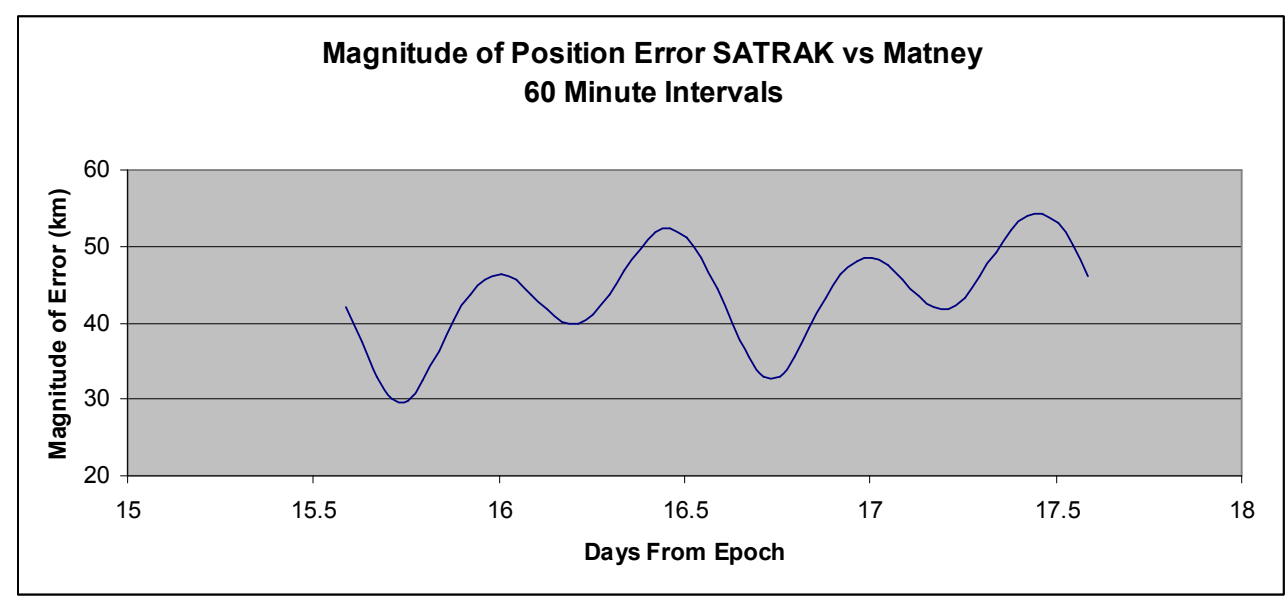

Figure 52 - 30000 Position Error Matney (60 min intervals)

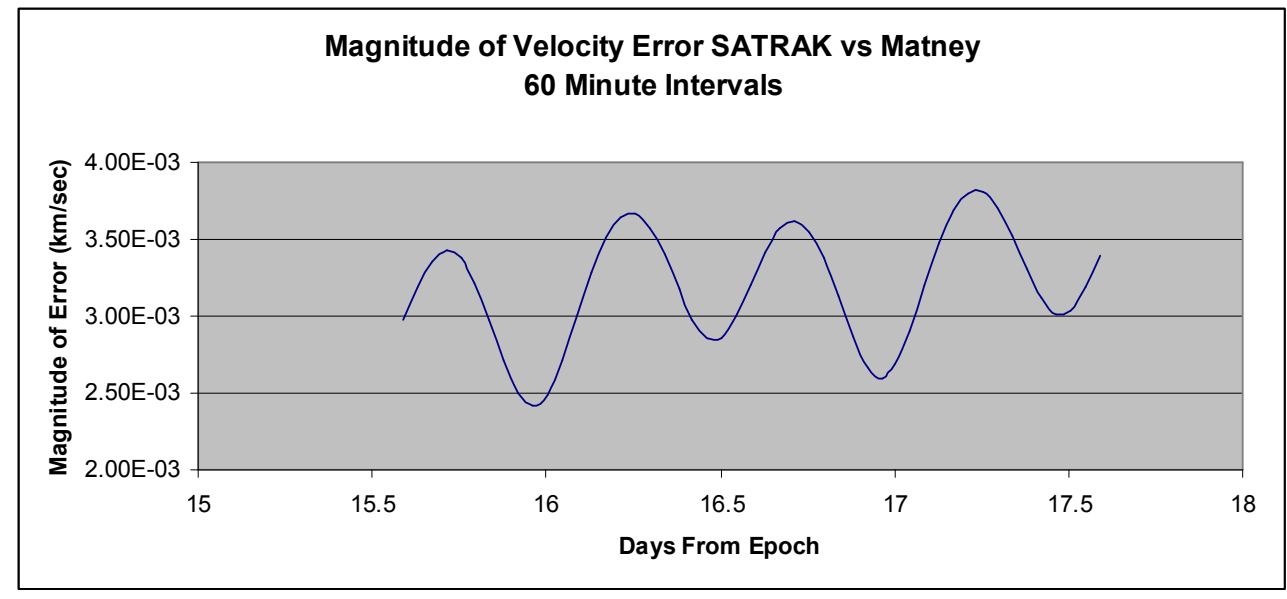

Figure 53 - 30000 Velocity Error Matney (60 min intervals) 


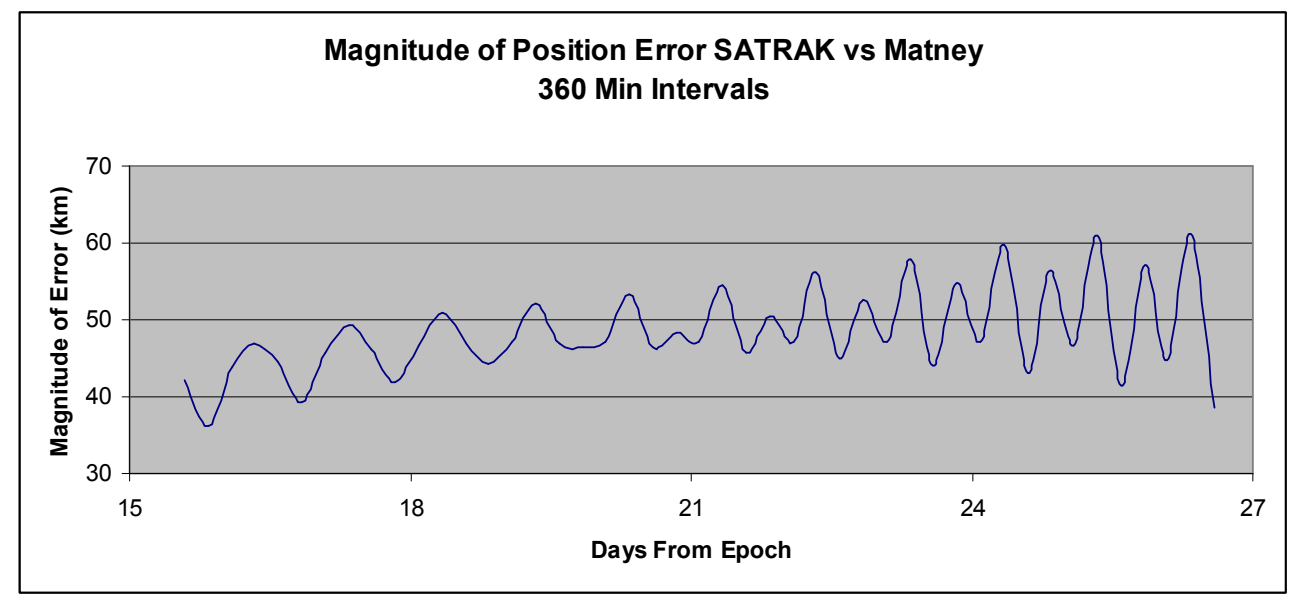

Figure 54 - 30000 Position Error Matney (360 min intervals)

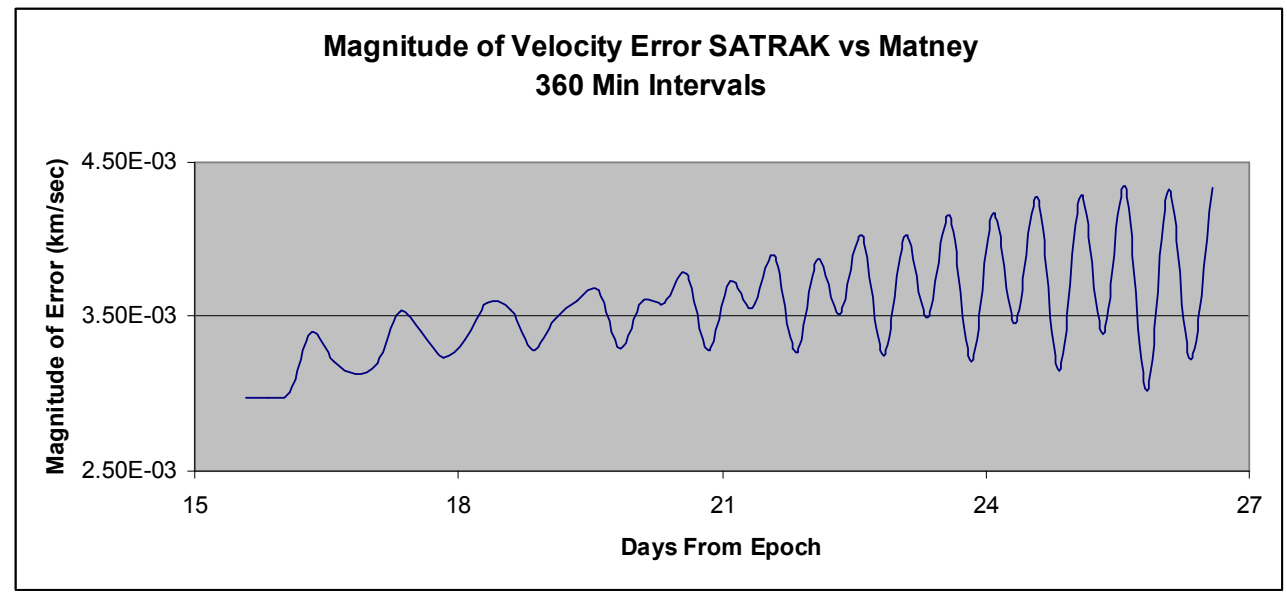

Figure 55 - 30000 Velocity Error Matney (360 min intervals)

\subsubsection{Analysis - JSC Code}

From visual inspection and direct comparison with the three previous codes, it is easy to see that the JSC code does not provide the same level of accuracy in the output. It is suspected that there are two main reasons for this. First, it is believed that there is a fundamental flaw in the programming of Kepler's solution. This is skewing output results even before any perturbations are taken into account. Second, the JSC code does 
not account for any third body interactions. This causes the periodic variations seen in the graphs, as sometimes the third body effects help the simulation while at other times it hurts the simulation.

Furthermore, the scope of the error is of similar magnitude to the threshold, and thus cannot be ignored like the previous three cases. This will be discussed further in Section 5.9 Operational Issues.

\subsection{Polysat}

Polysats are small satellites designed by Cal Poly under the Cube Sat project.. CP3 and CP4 were launched into $640 \mathrm{~km}$ sun-synchronous orbits on April 17, 2007. For the Polysats, there were two sets of truth data. First, there was SATRAK data of the CP3 available. Second, there was TLE data provided by Celestrak. Using archived and current TLE data, it is possible to see how far away the prediction is from official data.

For this analysis, only the modified code was compared to truth data. This is to check the operation potential of the new software beyond GEO objects. The original code was not tested because it was not designed for LEO or MEO objects. 


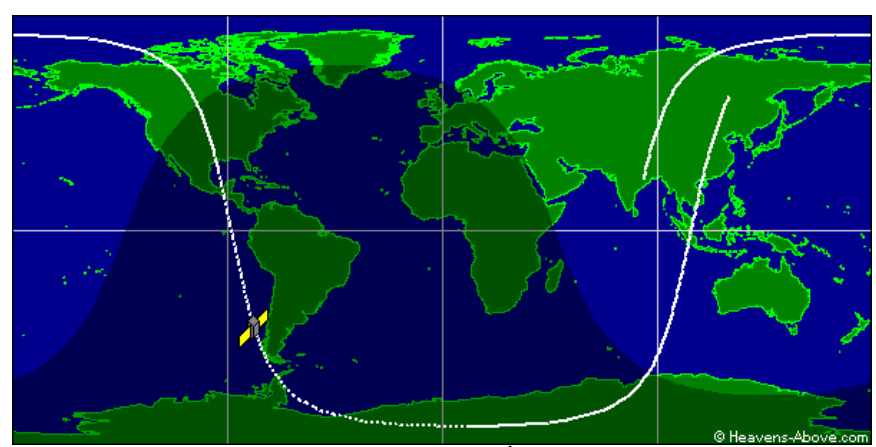

Figure 56 - CP3 Graphical Orbit Datav

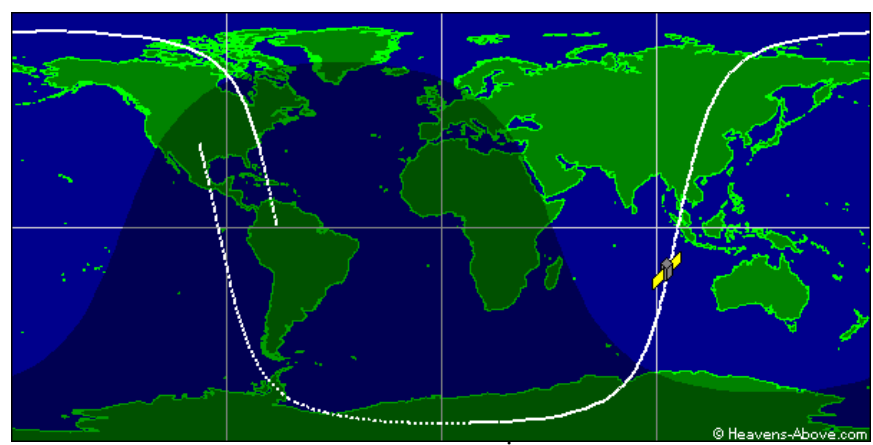

Figure 57 - CP4 Graphical Orbit Data ${ }^{\text {vi }}$
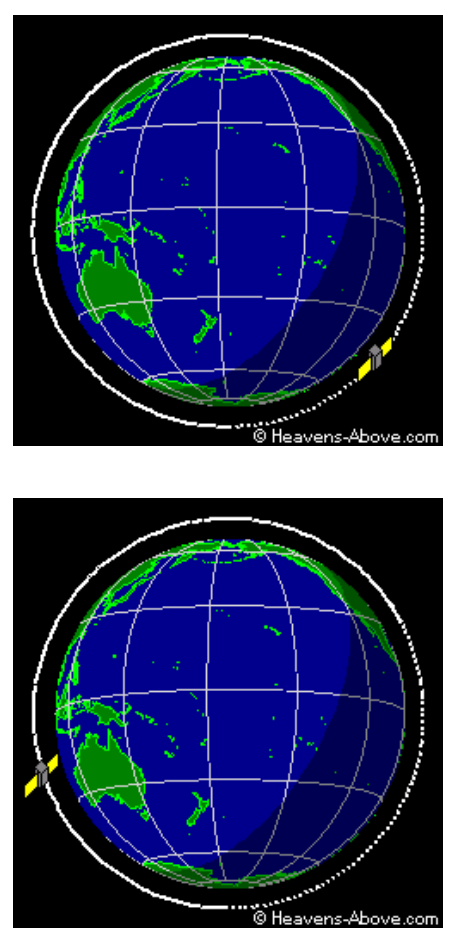

\subsubsection{CP3}

Below are graphs showing the magnitude of the vector error in both position and velocity of the modified code compared to SATRAK truth data. 


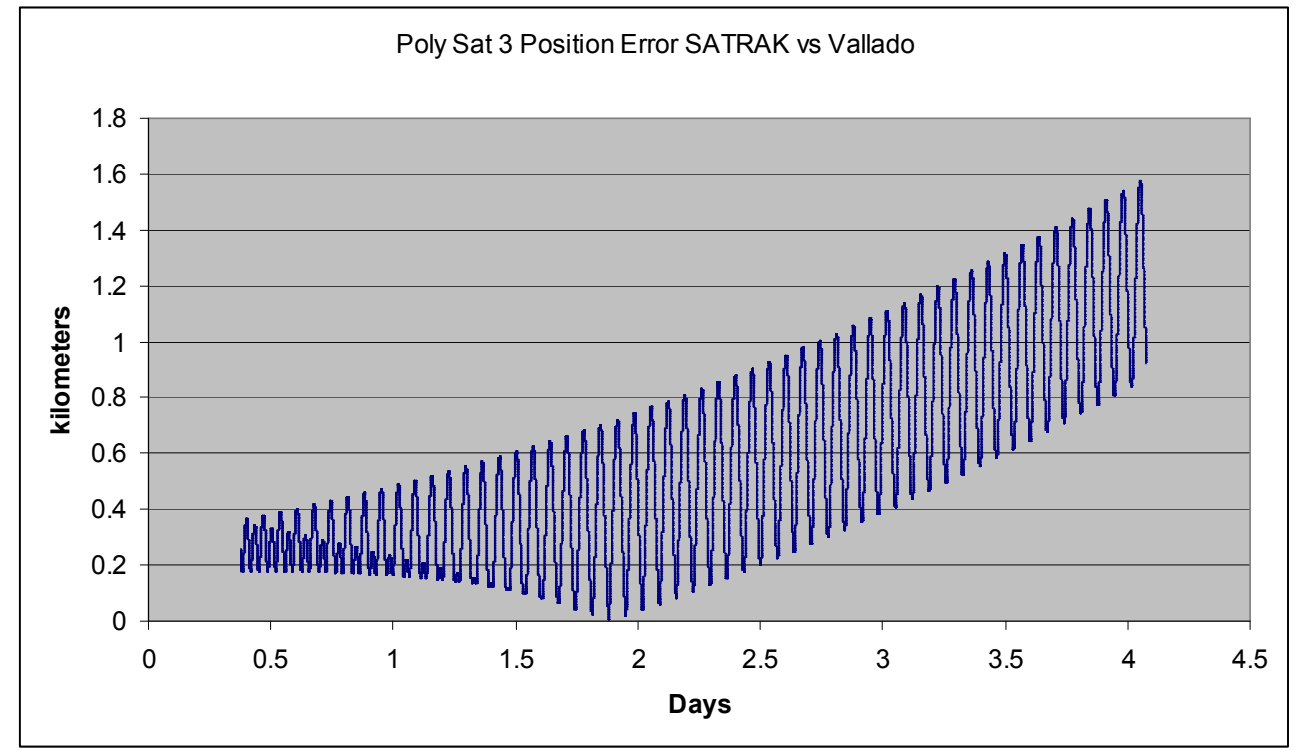

Figure 58 - CP3 Position Error Vallado (10 sec intervals)

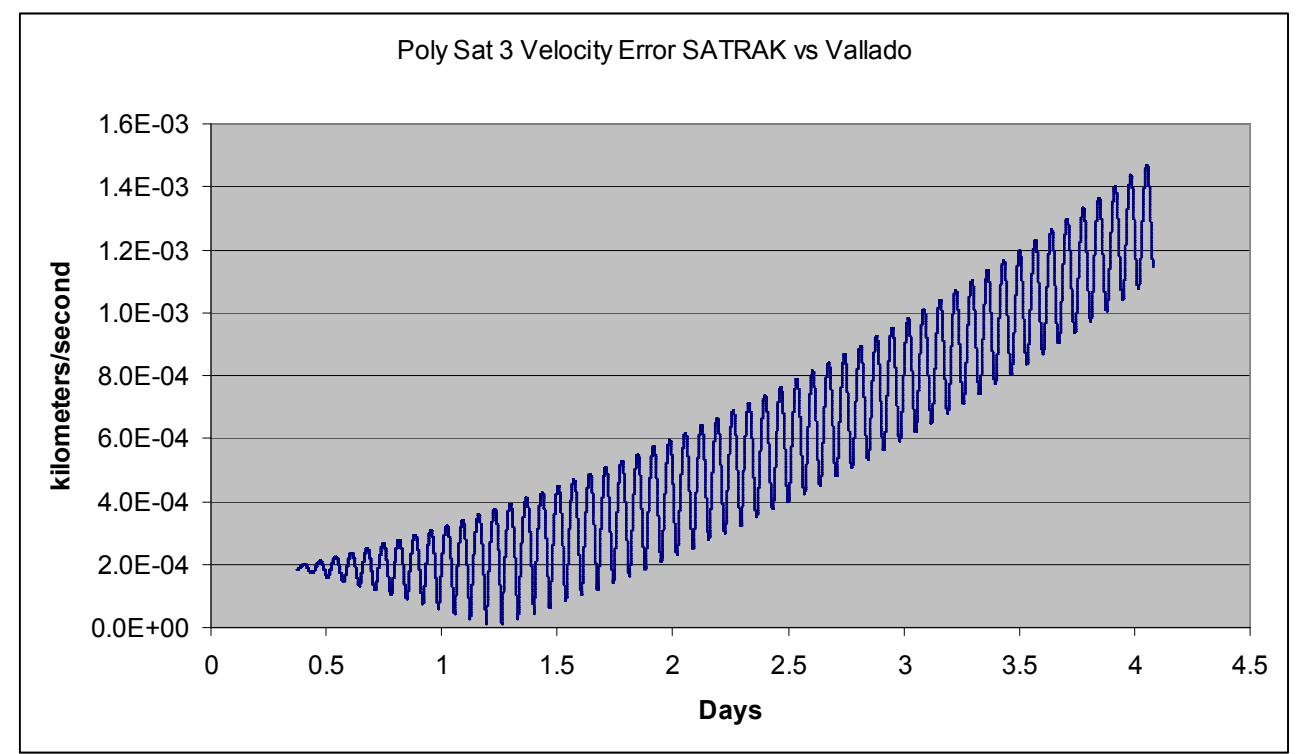

Figure 59 - CP3 Velocity Error Vallado (10 sec intervals)

These graphs show two trends. First, the variation in error is a function of the objects period and not of the Earth's rotation. The periodic motion of both the position and velocity corresponds to the mean motion of the spacecraft. Second, the slope of the error 
trends is also proportional to the mean motion of the object. In other words, the faster the object is moving, the faster error will be a significant factor.

Beyond SATRAK, there is also TLE data that can be used as truth data. TLE's were pulled from Celestrak at four different times and are shown in the table below.

\begin{tabular}{|c|c|}
\hline Date of Reading & TLE \\
\hline $4 / 22 / 09$ & 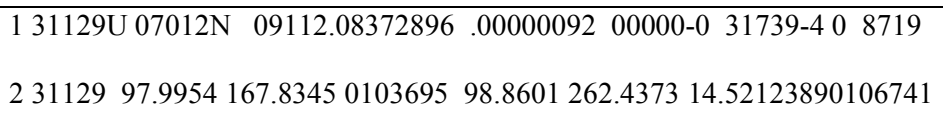 \\
\hline $5 / 1 / 09$ & 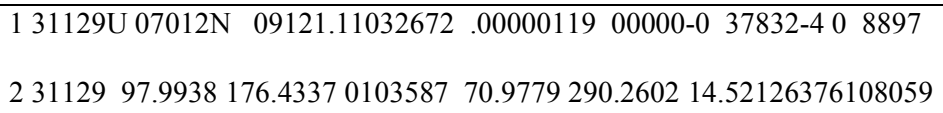 \\
\hline $5 / 6 / 09$ & 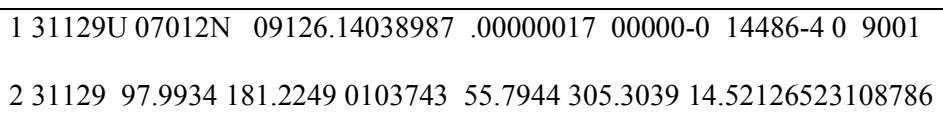 \\
\hline $5 / 23 / 09$ & 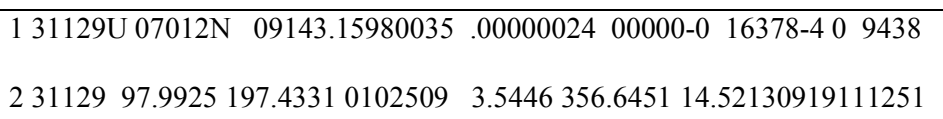 \\
\hline
\end{tabular}

Table 2 - CP3 TLE Results

\begin{tabular}{|l|l|l|}
\hline Days since 4/22 TLE & Error in Position $(\mathrm{km})$ & Error in Velocity $(\mathrm{km} / \mathrm{sec})$ \\
\hline 9.03 & 0.638 & 0.008662 \\
\hline 14.06 & 2.802 & 0.002583 \\
\hline 31.08 & 9.197 & 0.000703 \\
\hline
\end{tabular}

These results are significantly better than expected given the SATRAK analysis. This shows that SATRAK data and TLE data do not necessarily match. Based on this, the modified code may give more accurate results than SATRAK. If the new code contains 
more modeled perturbations than SATRAK, this would also explain the periodic variations seen in most of the preceding figures.

\subsubsection{CP4}

Table 3 - CP4 TLE Data

\begin{tabular}{|c|c|}
\hline Date of Reading & TLE \\
\hline $4 / 22 / 09$ & 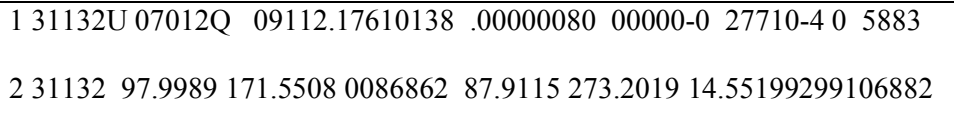 \\
\hline $5 / 1 / 09$ & 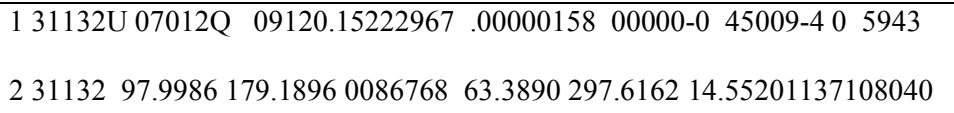 \\
\hline $5 / 23 / 09$ & $\begin{array}{l}131132 \mathrm{U} 07012 \mathrm{Q} \quad 09143.18655556-.0000023100000-0-40476-406101 \\
231132097.9973201 .24220085319352 .7051007 .289914 .55204047111390\end{array}$ \\
\hline
\end{tabular}

Table 4 - CP4 Results

\begin{tabular}{|l|l|l|}
\hline Days since 4/22 TLE & Error in Position $(\mathrm{km})$ & Error in Velocity $(\mathrm{km} / \mathrm{sec})$ \\
\hline 7.98 & 0.5924 & 0.000514 \\
\hline 31.01 & 12.253 & 0.0115 \\
\hline
\end{tabular}

The $\mathrm{CP} 4$ results are similar to the $\mathrm{CP} 3$ results. The error in both position and velocity are of similar magnitude after similar amounts of time. From this, a general rate of error propagation can be assessed.

\subsection{ISS Tool bag}

The ISS Tool bag was a piece of debris dropped by astronauts working on the outside of the International Space Station. The ISS is in orbit at approximately $350 \mathrm{~km}$ altitude. 
The ISS Tool bag is currently in orbit at approximately $300 \mathrm{~km}$ altitude due to drag perturbations and lack of station keeping.
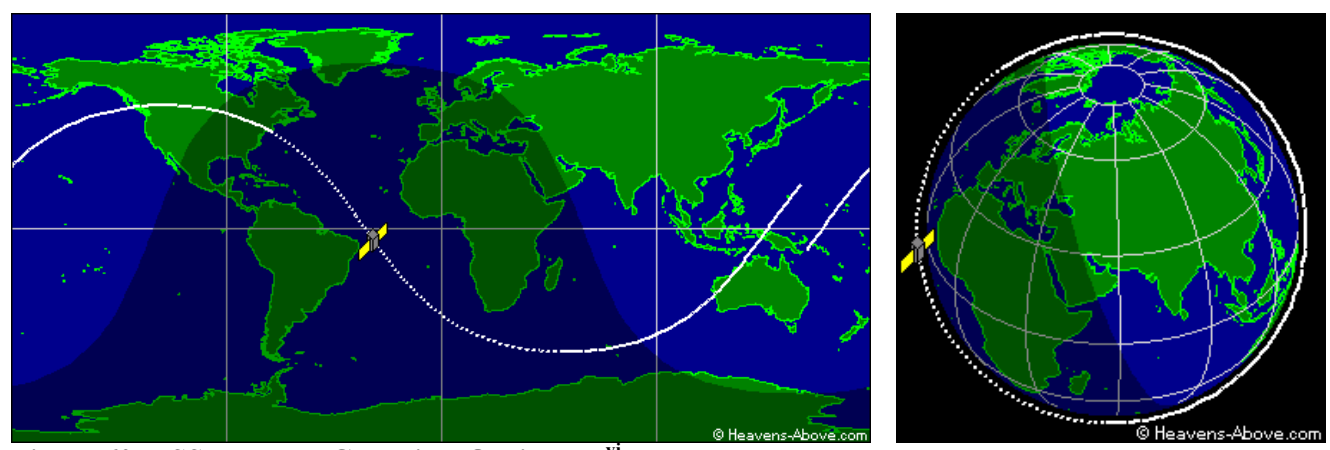

| Figure 60 - ISS Tool_bag Graphical Orbit Data ${ }^{\text {vi }}$

The following TLE's were downloaded from Celestrak.

Table 5 - ISS Tool bag TLE Data

\begin{tabular}{|c|c|}
\hline Date of Reading & TLE \\
\hline $5 / 1 / 09$ & 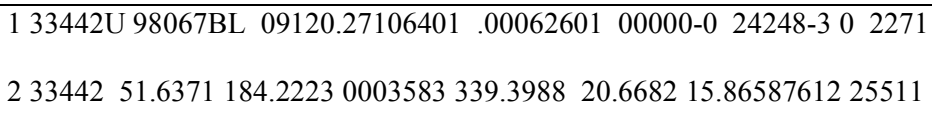 \\
\hline $5 / 6 / 09$ & 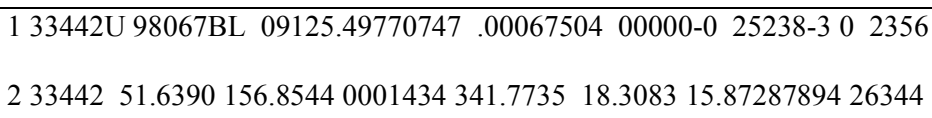 \\
\hline $5 / 23 / 09$ & 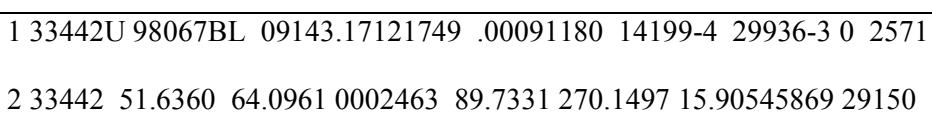 \\
\hline
\end{tabular}

Table 6 - ISS Tool bag Results

\begin{tabular}{|l|l|l|}
\hline Days since 4/22 TLE & Error in Position $(\mathrm{km})$ & Error in Velocity $(\mathrm{km} / \mathrm{sec})$ \\
\hline 5.23 & 33.645 & 0.0388 \\
\hline 22.90 & 3041.2 & 3.519 \\
\hline
\end{tabular}


The SGP4 propagator breaks down quickly when dealing with extremely low orbits.

After 5 days, the error in position has already become significant and after 23 days, the projection is useless. The error in the projection is caused by an ever changing $\mathrm{B}^{*}$ term in the TLE, or the term used to approximate drag. The force of drag is changing constantly due to the object falling further and further into the atmosphere. Propagation for LEO objects should be limited to short periods of time using the new code.

\subsection{Iridium Debris}

Iridium Debris is a piece of the Iridium 33 satellite that broke up in LEO. The altitude of the debris is approximately $775 \mathrm{~km}$, putting it in a similar orbit to the Polysats.
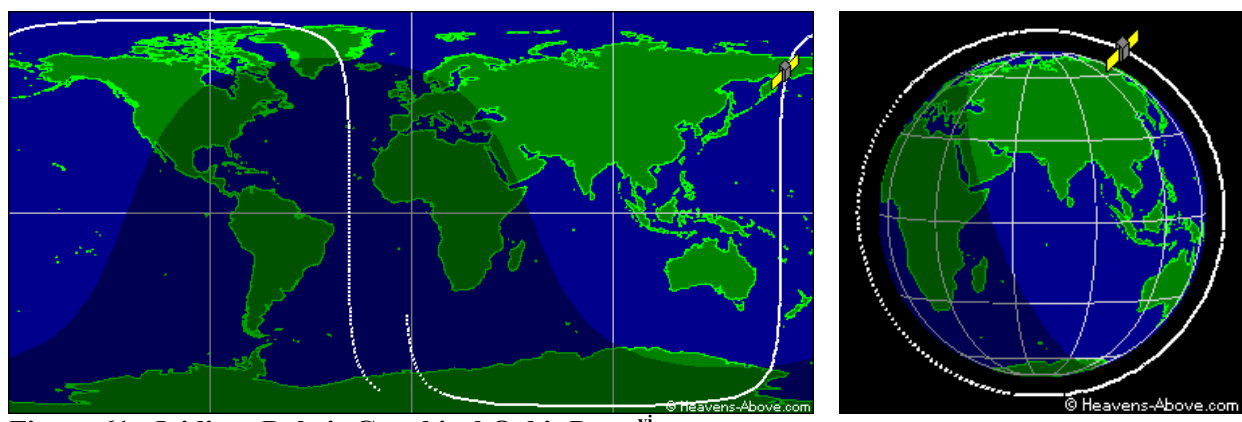

Figure 61 - Iridium Debris Graphical Orbit Data

Table 7 - Iridium Debris TLE Data

\begin{tabular}{|c|c|}
\hline Date of Reading & TLE \\
\hline $4 / 22 / 09$ & 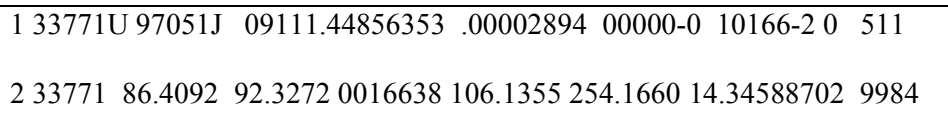 \\
\hline $5 / 1 / 09$ & $\begin{array}{llllllll}133771 U & 97051 \mathrm{~J} & 09120.30665597 & .00004031 & 00000-0 & 14163-2 & 0 & 587 \\
233771 & 86.4081 & 88.6364 & 0017979 & 83.2643 & 277.0615 & 14.34660268 & 11251\end{array}$ \\
\hline $5 / 23 / 09$ & 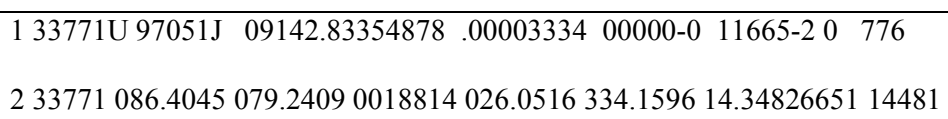 \\
\hline
\end{tabular}


Table 8 - Iridium Results

\begin{tabular}{|l|l|l|}
\hline Days since 4/22 TLE & Error in Position $(\mathrm{km})$ & Error in Velocity $(\mathrm{km} / \mathrm{sec})$ \\
\hline 8.59 & 41.315 & 0.0444 \\
\hline 31.39 & 470.04 & 0.4969 \\
\hline
\end{tabular}

\subsection{Operational Issues}

There are two main issues to consider when investigating whether or not to switch to the new system. First, how will the improvements in the code affect the program effectiveness? Second, how will the additions to the code affect the speed of operation?

\subsubsection{Program Effectiveness}

Working with the assumption that the original JSC code provides 'good enough' answers, the following estimates how long the JSC code could successfully track the satellite. If this range of time falls within the scope of program operations, then JSC would not be able to differentiate between the old and new SGP4 versions.

To calculate this estimation, there were some simplifying assumption made. First, NASA uses a telescope with a 2 degree square FOV to initially find the object. It then uses the PREDICT software to try to find the same object with a 0.2 degree square FOV telescope. For this estimation, a 0.2 degree round FOV was assumed. This means that 
the threshold for the angle away from the position truth vector is half of this, or 0.1 degrees. Second, the angle from the center of the Earth was calculated rather than from some point on the surface of the Earth. This assumption has both the ability to help or harm the prediction based on the vector error and where the telescope actually is, but should give a reasonable answer. Third, since SATRAK data was not available for all the points needed, the MATLAB data was used as truth. Earlier tests showed that MATLAB provided accurate outputs consistently.

Below are graphs of the three objects defined by NASA JSC showing the angle at which the object is from where the old SGP4 program tells the telescope to point. A threshold line of 0.1 degrees is also drawn for a point of comparison.

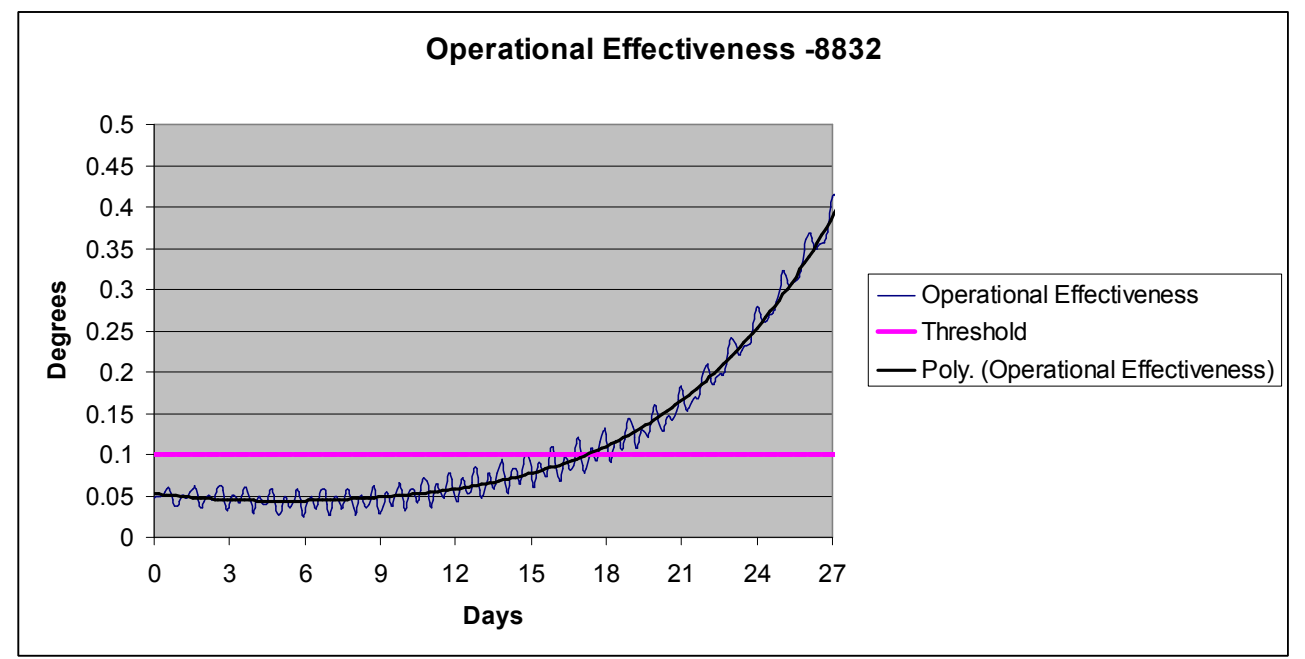

Figure 62 - 8832 Operational Effectiveness 


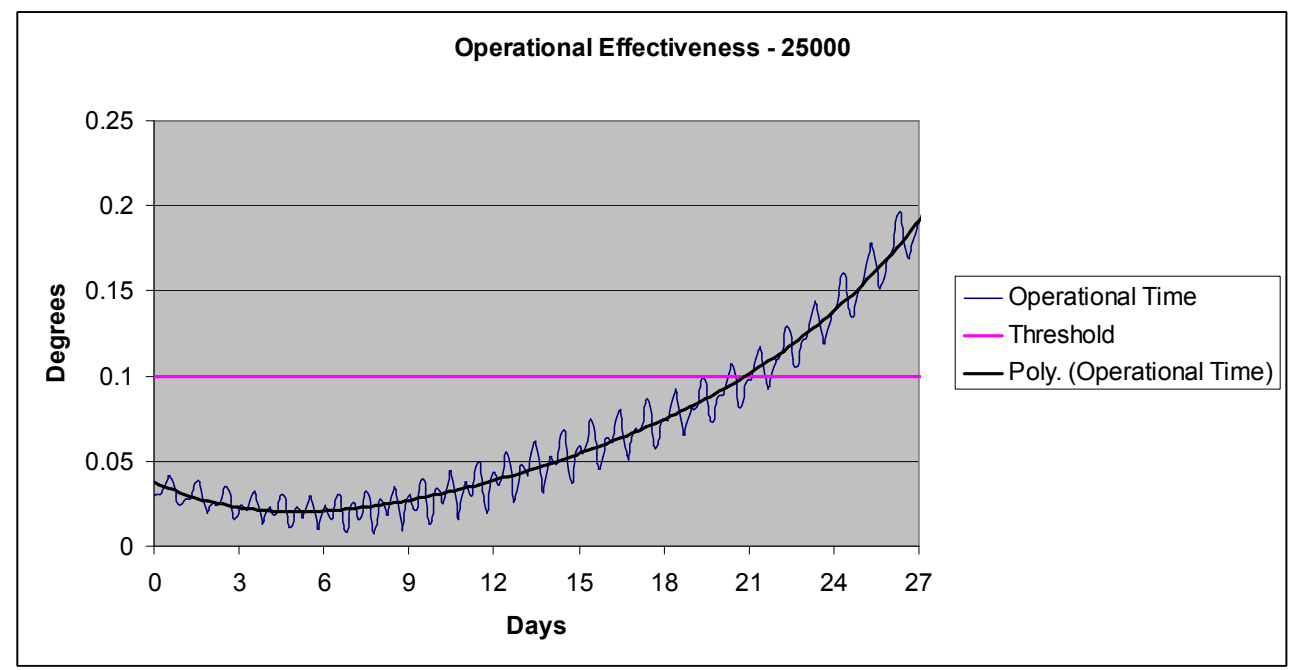

Figure 63 - 25000 Operational Effectiveness

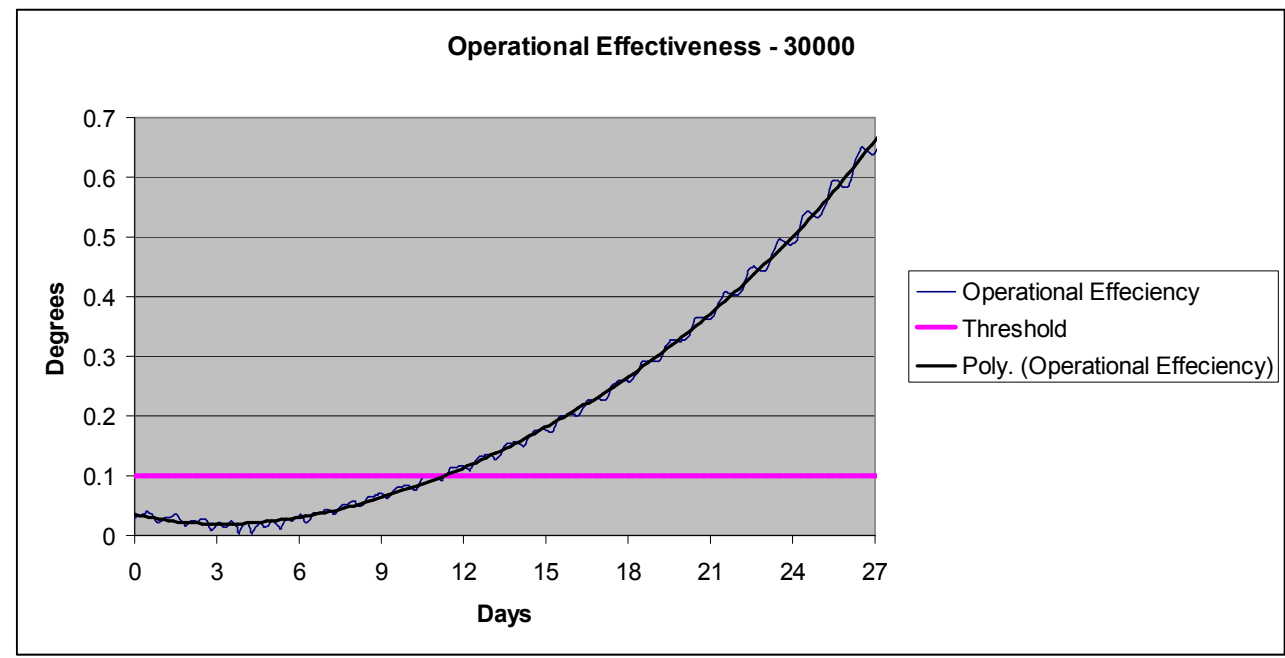

Figure 64 - 30000 Operational Effectiveness

All three cases show that the existing SGP4 propagator breaks down and is no longer effective after a matter of days. From these limited examples, it takes 12 days for the first object to leave the FOV. Furthermore, these examples show that the propagator is most effective between three and nine days. What is troubling about these graphs, 
mentioned briefly earlier, is the fact that at time zero, the telescope will not be centered on the target. Again, it is believed this to be caused by a flaw in programming Kepler's solution. The above graphs can be compared to the following:

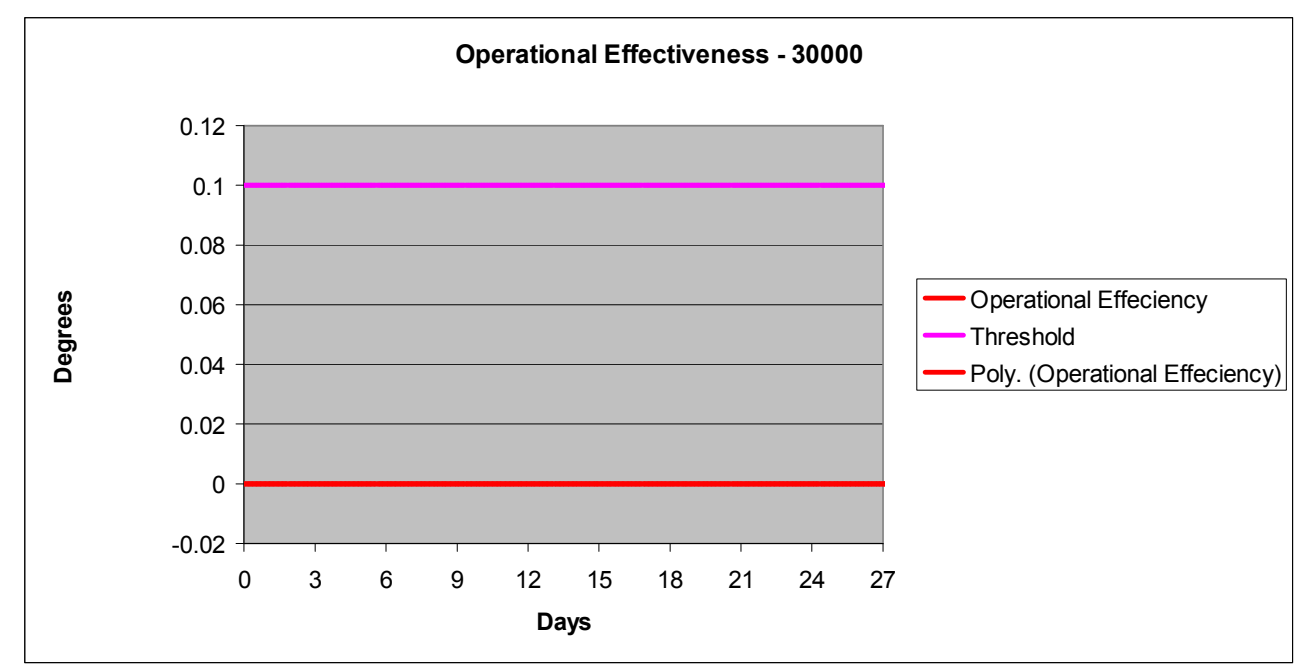

Figure 65 - 30000 Operational Effectiveness (new code)

The error with the new code is essentially zero throughout the entirety of the simulation and is difficult to read in the above chart. Object 30000 offered the poorest results and thus was chosen as a point of comparison. The new code offers significant improvement over the old code and will stay within threshold limits indefinitely. Additionally, even in short term situations, the new code offers superior accuracy.

Below is the operational effectiveness of the new code in relation to Polysat 3 (CP3): 


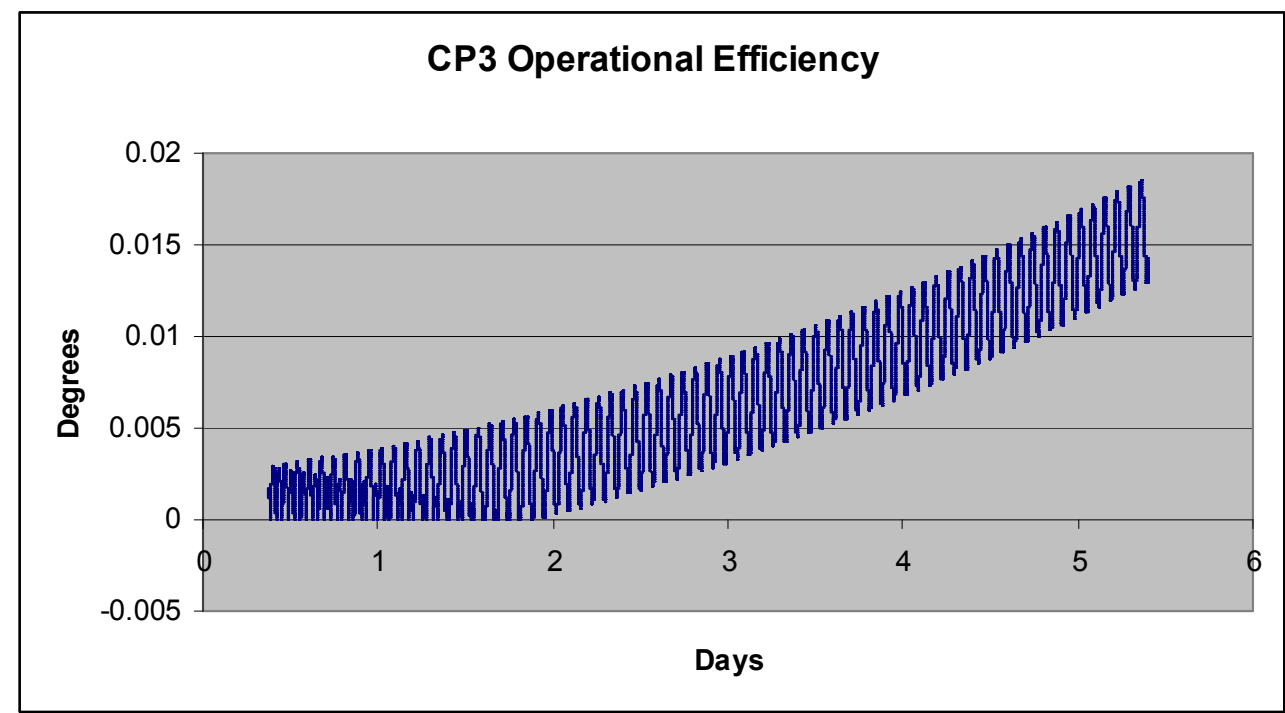

Figure 66 - CP3 Operational Effectiveness

Though the values only extend to roughly 5 days, extrapolating the data using a quadratic suggests that the modified code will be able to keep the object in the FOV for roughly 15 days. Using the TLE truth data points to a different model. Using data between 4/22/09 and 5/23/2009 which is roughly 31 days, shows that the object will be about .071 degrees away from the center of the FOV - still within threshold limits. This shows that the growth in error is linear instead of polynomial, suggesting that the modified code will be able to keep the object in the FOV for roughly 40 days.

\subsubsection{Operational Speed}

Comparing lines of code, the new program contains 4475 lines with comments and blank lines, while the existing code only contains 2510 lines. No noticeable difference was encountered when running the two programs. 


\section{Conclusion}

The preceding results lead to the following conclusions:

In reference to the original question of whether or not NASA JSC should change their system to incorporate the new Vallado code, the answer is yes. This should be done for two distinct reasons. First, the modified code is more accurate by orders of magnitude over a much longer period of time. Second, the old code provides flawed answers off by kilometers even at time zero. Though the old code provides 'good enough' answers for periods up to about one week, this assumes that the input data is accurate. In reality, the nature of the input data may contain significant errors as well. This is because the code uses right ascension and declination data separated by brief periods of time (less than 5 minutes), thus the object being followed does not move far and the right ascension and declination change only slightly. Calculating COES from this data is limited to the accuracy and the significant figures provided by the telescope data. In such, the 'good enough' answers provided by the old code may not be 'good enough' when compounded with errors in telescope readings.

From the analysis, it is apparent that Vallado's code does not exactly match the output of SATRAK, though it is close. The periodic nature of the error between the two codes, which mimics the period of the orbiting object around the Earth, implies that there are perturbations or constants that differ between the two programs. For this analysis, SATRAK data is taken as truth; however, the error in the SATRAK program is unknown. It is possible that Vallado better predicts the position and velocity of orbiting objects. For the purposes of this project, the two outputs are statistically identical. 
If using the Vallado code to replace SATRAK, the MATLAB version of the code is more reliable than the FORTRAN version. Even though the two programs yield indistinguishable results, the MATLAB version of the code is more intuitive and easier to follow. Furthermore, FORTRAN proved more difficult to use as the program balked at times for unknown reasons. Both programs have been provided for use on the attached CD with a User's Guide in section 7.

Finally, the modified code has limitations based on the altitude of the satellite. For objects in deep space orbits such as GEO, the code accurately predicts (to the same level as SATRAK) the position and the velocity of the object within threshold limits indefinitely. In MEO, the effectiveness drops to roughly two weeks of predictions within the threshold limits. In LEO, the effectiveness drops to about one day of predictions within the threshold limits. Thus, the new code will work for all orbiting objects; however, it is important to keep in mind the operational timeline of activities.

Furthermore, for LEO and MEO objects, the B* term needs to be added to the system, or else the accuracy of the results may decrease. Improvements to the PREDICT code must be made to accommodate this before use. 


\section{User's Guide}

This section provides instructions on how to install and run the three different codes discussed in this paper. Sections 7.1 and 7.2 describe the SATRAK replacement codes written in MATLAB and FORTRAN. Section 7.3 describes how to incorporated the modified code into the existing NASA JSC PREDICT code as well as the various options the user has within the code itself.

\subsection{MATLAB}

**Note that the output file will be over-written each time the program is run unless the filename is changed.**

The MATLAB code mimics the SATRAK code and outputs very similar results. Work was done using MATLAB 7.0.

\subsubsection{Installation}

On the attached CD, there is a folder named SGP4 MATLAB. Copy and paste this folder to MATLAB's current directory. The folder contains the following files:

- testmat.m Driver script for testing and example usage

- $\quad$ sgp4.m Main sgp4 routine

- sgp4init.m Initialization routine for sgp4

- initl.m Initialization for sgp4

- dsinit.m Deep space initialization 
- dspace.m

- dpper.m

- dscom.m

- twoline2rv.m

- angl.m

- constmath.m

- days 2 mdh.m

- getgravc.m

- gstime.m

- invjday.m

- jday.m

- mag.m

- newtonnnu.m

- rv2coe.m

- SGP4-VER.TLE Verification file

- Sgp4_CodeReadme.pdf

elements
Deep space perturbations

Deep Space periodics

Deep Space common variables

TLE conversion routine

Find the angle between two vectors

set mathematical constants

convert days to month day hour minute second

Get the gravity constants

Find Greenwich sidereal time

Inverse Julian Date

Find the Julian Date

Magnitude of a vector

Kepler's iteration given eccentricity and true anomaly

Convert position and velocity vectors to classical orbital

\subsubsection{Verification Run}

1. Run testmat.m

2. Enter opsmode 'i' for improved method

3. Enter typerun ' $v$ ' for verification mode 
4. Enter constant ' 72 ' corresponding to WGS-72 standards

5. Enter file name 'SGP4-VER.TLE' - see appendix 1 for file information

6. The program should output a file called 'tmatver.out' can be compared to the results found in appendix 2 for verification that the program is running properly.

\subsubsection{TLE inputs}

The program does not discriminate against file types when opening TLEs, though it has only been tested with .TXT and .TLE file extensions. Make sure that the input TLE does have proper the proper format. A simple way to load a TLE is to open a new script in MATLAB and copy and paste the desired TLE, then save the script with a TLE file extension.

The program is capable of handling multiple TLEs in a single run and will output the file with a header representing the satellite number before each set of data. When formatting a multiple TLE input, use a '\#' symbol to begin each line that does not contain TLE data. This tells the computer to skip the line and move to the next line.

\subsubsection{Catalog Mode}

Catalog mode takes a TLE input and outputs data for -24 hours to +24 hours with 20

minute intervals. This is useful for short term propagations of many objects (i.e. multiple TLEs).

1. Run 'testmat.m' 
2. Enter ' $a$ ' for afspc or ' $\mathrm{i}$ ' for improved method

3. For type of run, enter 'c' for catalog mode

4. Enter TLE data - file must be completely written with the file extension name

5. A new file should be created with the name 'tmatall.out' with the catalog data.

\subsubsection{Manual Mode}

Manual mode gives the user the opportunity to define when to start and stop calculating and at what time intervals. To do this, the user is given three options to define time:

- Minutes from epoch

- Epoch

- Day of Year

The minutes from epoch approach assumes that the epoch is defined by the time stated within the TLE. Simply input (in minutes) how much time you want to elapse before the first data point, and how much time you want to elapse before the last data point. The epoch approach asks for a start date and time and a stop date and time. Finally, the day of year approach asks for a year and a day of the year. The day of the year can be in decimal form to indicate a fraction of a day. For all three approaches, the reference time frame is UTC. Furthermore, whatever mode chosen will prompt for the time step that will be used (in minutes).

**Note, when working with multiple TLEs in manual mode, the user will be prompted for start and stop points and time step for each individual TLE.** 
1. run 'testmat.m'

2. Enter ' $a$ ' for afspc or ' $i$ ' for improved mehod

3. For type of run, enter ' $m$ ' for manual operation

4. Enter how the start and stop points are determined
a. $\mathrm{m}=$ minutes from epoch
b. $\mathrm{e}=$ epoch (year, month, day, hour, min, sec)
c. d= day of year (year, day)

5. Enter constant choice ( 72 gives answers closest to truth)

6. Enter TLE filename

7. Follow prompts on screen to input start and stop points and time step

8. Program sends output to file named 'tmat.out'

\subsubsection{Understanding the Output File}

The output comes without column headers, so it is important to know what the values represent. The first line is a header line with information about the satellite number. When working with multiple TLEs, data from each TLE is separated by a similar header with the satellite number. For each satellite, there are 7-13 columns of data (columns 813 are date and time data which is not calculated for catalog mode):

$\begin{array}{ll}\text { Column 1 } & \text { Minutes from Epoch } \\ \text { Column 2 } & \text { X-Position } \\ \text { Column 3 } & \text { Y-Position }\end{array}$




$\begin{array}{ll}\text { Column 4 } & \text { Z-Position } \\ \text { Column 5 } & \text { X-Velocity } \\ \text { Column 6 } & \text { Y-Velocity } \\ \text { Column 7 } & \text { Z-Velocity } \\ \text { Column 8 } & \text { Year } \\ \text { Column 9 } & \text { Month } \\ \text { Column 10 } & \text { Day } \\ \text { Column 11 } & \text { Hour } \\ \text { Column 12 } & \text { Minute } \\ \text { Column 13 } & \text { Second }\end{array}$

**Note, the position vector is based in ECI coordinate frame.**

\subsection{FORTRAN (Vallado Code)}

The main FORTRAN program has many similarities with the MATLAB program and may reference certain sections.

**Note that the output file will be over-written each time the program is run unless the filename is changed.** 


\subsubsection{Installation}

On the attached CD, there is a folder labeled SGP4 FORTRAN. Copy and paste the folder to a working directory. The files contained in the folder are:

$\begin{array}{ll}\text { ASTMATH.CMN } & \text { Common file with mathematical constants } \\ \text { SGP4.CMN } & \text { Common file with constants applicable to SGP4 model } \\ \text { TESTFOR.FOR } & \text { Fixed Format FORTRAN file } \\ \text { TESTFOR.EXE } & \text { Executable file of TESTFOR.FOR } \\ \text { Validation.OUT } & \text { Data to validate correct installation } \\ \text { SGP4-VER.TLE } & \text { Validation TLE Data }\end{array}$

Either use the executable (compiled and built on Silverfrost), or compile and build the FORTRAN file using another compiler. Correct installation should result in the program running in a command prompt.

\subsubsection{Verification Run}

** FORTRAN is case sensitive, please keep this in mind when entering text.**

1. Run TESTFOR.EXE

2. Select opsmode 'i'

3. Select run type ' $v$ '

4. Input filename 'SGP4-VER.TLE'

5. Program will output a file named 'tfor.out' to the working directory 
6. Compare this output with data from the file labeled 'Verification.OUT'

\subsubsection{TLE Input}

This function is identical to MATLAB. See section 7.1.3.

\subsubsection{Catalog Mode}

Catalog mode takes a TLE input and outputs data for -24 hours to +24 hours with 10

minute intervals. This is useful for short term propagations of many objects (i.e. multiple TLEs).

1. Run 'TESTFOR.EXE - This should bring up a command prompt

2. Enter 'a' for afspc or 'i' for improved method

3. For type of run, enter 'c' for catalog mode

4. Enter TLE filename- file must be completely written with the file extension name

5. A new file should be created with the name 'tfor.out' with the catalog data.

\subsubsection{Manual Mode}

Manual mode gives the user the opportunity to define when to start and stop calculating and at what time intervals. To do this, the user is given three options to define time:

- Minutes from epoch

- Epoch

- Day of Year 
The minutes from epoch approach assumes that the epoch is defined by the time stated within the TLE. Simply input (in minutes) how much time you want to elapse before the first data point, and how much time you want to elapse before the last data point. The epoch approach asks for a start date and time and a stop date and time. Finally, the day of year approach asks for a year and a day of the year. The day of the year can be in decimal form to indicate a fraction of a day. For all three approaches, the reference time frame is UTC. Furthermore, whatever mode chosen will prompt for the time step that will be used (in minutes).

**Note, when working with multiple TLEs in manual mode, the user will be prompted for start and stop points and time step for each individual TLE.**

9. run 'TESTFOR.EXE' - a new command prompt should appear

10. Enter 'a' for afspc or 'i' for improved method

11. For type of run, enter ' $m$ ' for manual operation

12. Enter how the start and stop points are determined (Case sensitive)

a. $\mathrm{M}=$ minutes from epoch

b. $E=$ epoch (year, month, day, hour, min, sec)

c. $\mathrm{D}=$ day of year (year, day)

13. Enter constant choice (72 gives answers closest to truth)

14. Enter TLE filename

15. Follow prompts on screen to input start and stop points and time step 
16. Program sends output to file named 'tfor.out'

\subsubsection{Understanding the Output File}

The output file created is in the same format at the MATLAB output. Please see section 7.1.6 for more details.

\subsection{Modified FORTRAN Code - For JSC}

The modified FORTRAN code is designed to be compatible with the current PREDICT program; however, epoch data needs to be passed to the new program. This section does not describe how to run the PREDICT code. Rather, it explains how to integrate the modified code with the existing code.

\subsubsection{Installation}

On the attached $\mathrm{CD}$ in the folder 'MODIFIED CODE', there is a file called:

SGP4_Classic Subroutine.txt

Open this file and copy all of the contents. Next in the PREDICT source code, locate the SGP4_Classic subroutine (line 853 - line 1116 in Feb. version). Delete the entire subroutine and replace it with the text copied above.

To solve the epoch data problem, a global file called /global2/ is created in the surveychasenew subroutine. In the line following the definition of /global1/ (or line 4314 after addition of the new code) add the following line ( $\square$ represents a space): 


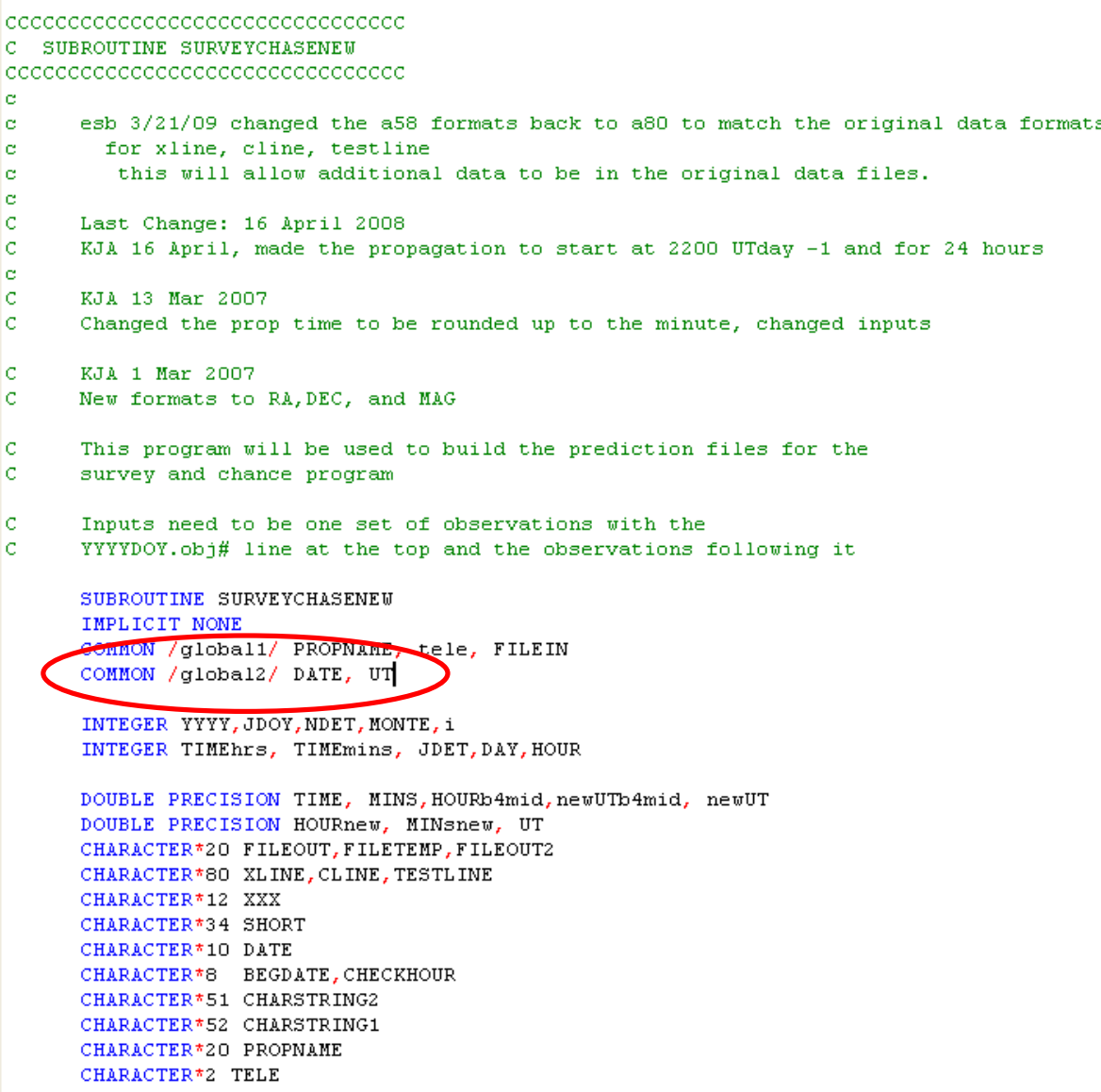

Figure 67 - Code Addition to SURVEYCHASENEW

Next, in the same directory on the $\mathrm{CD}$, there are two common files entitled:

- SGP4.CMN

\section{- ASTMATH.CMN}

Copy and paste these files into the directory with the PREDICT source code, as the new SGP4 propagator references these files.

Now, the updated PREDICT code will be ready to be compiled. 


\subsubsection{Code Options}

The Vallado code contains various options that have been pre-programmed for the Orbital Debris Program Office use; however, these can changed if necessary.

\subsubsection{Constant Values}

The program is set to use World Geodetic System (WGS) 72 parameters for Earth defined constants. These parameters are legacy values from the first code release of SGP4, and best match SATRAK truth data. The Vallado code is programmed to allow an update to the WGS 84 parameters, which are considered up-to-date until $2010^{\mathrm{vii}}$. To make this switch, find where 'whichconst' is defined in the SGP4_Classic subroutine ( line 925) and change the value from 72 to 84.

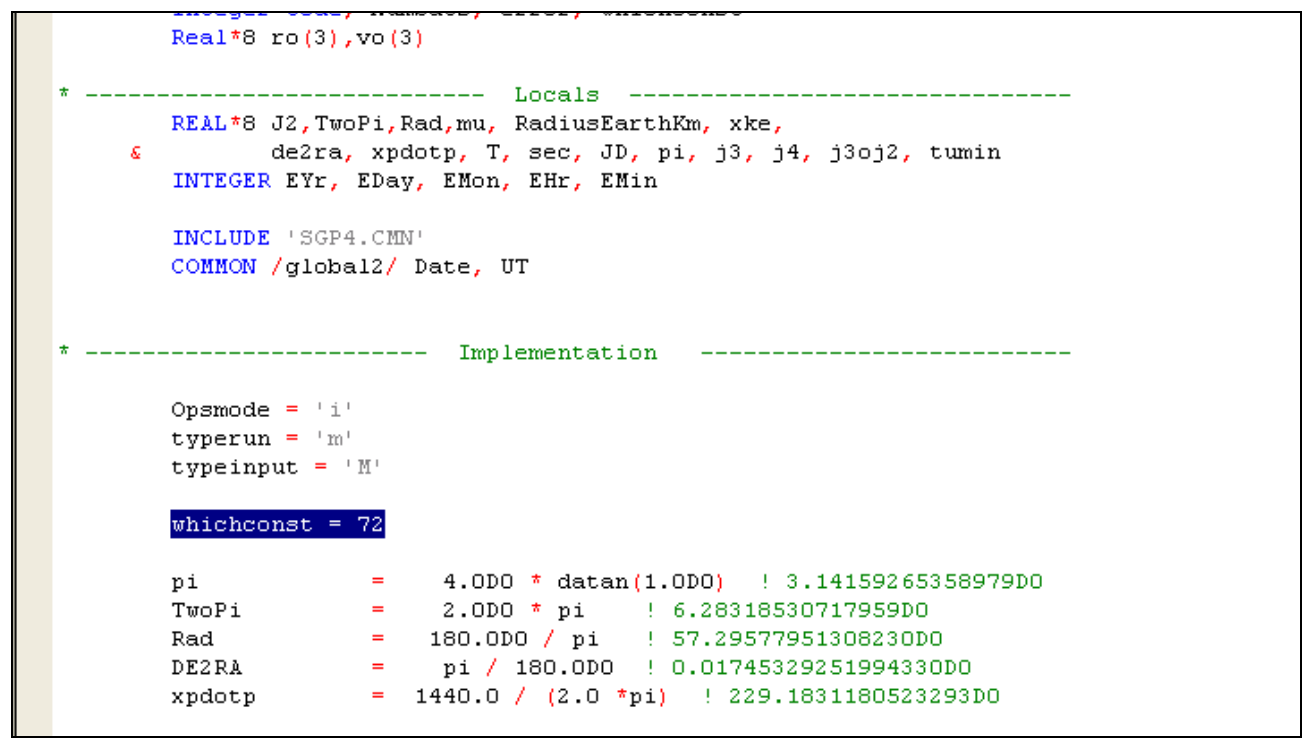

Figure 68 - Changing WGS values (1)

If manual changes are necessary to the constants, find the subroutine 'getgravconst'

( line 1012). The constants for specific called cases are listed in various IF statements. 
The user can define radius of Earth, J2, J3, and J4 harmonics for either the WGS 72 or

WGS 84 system.

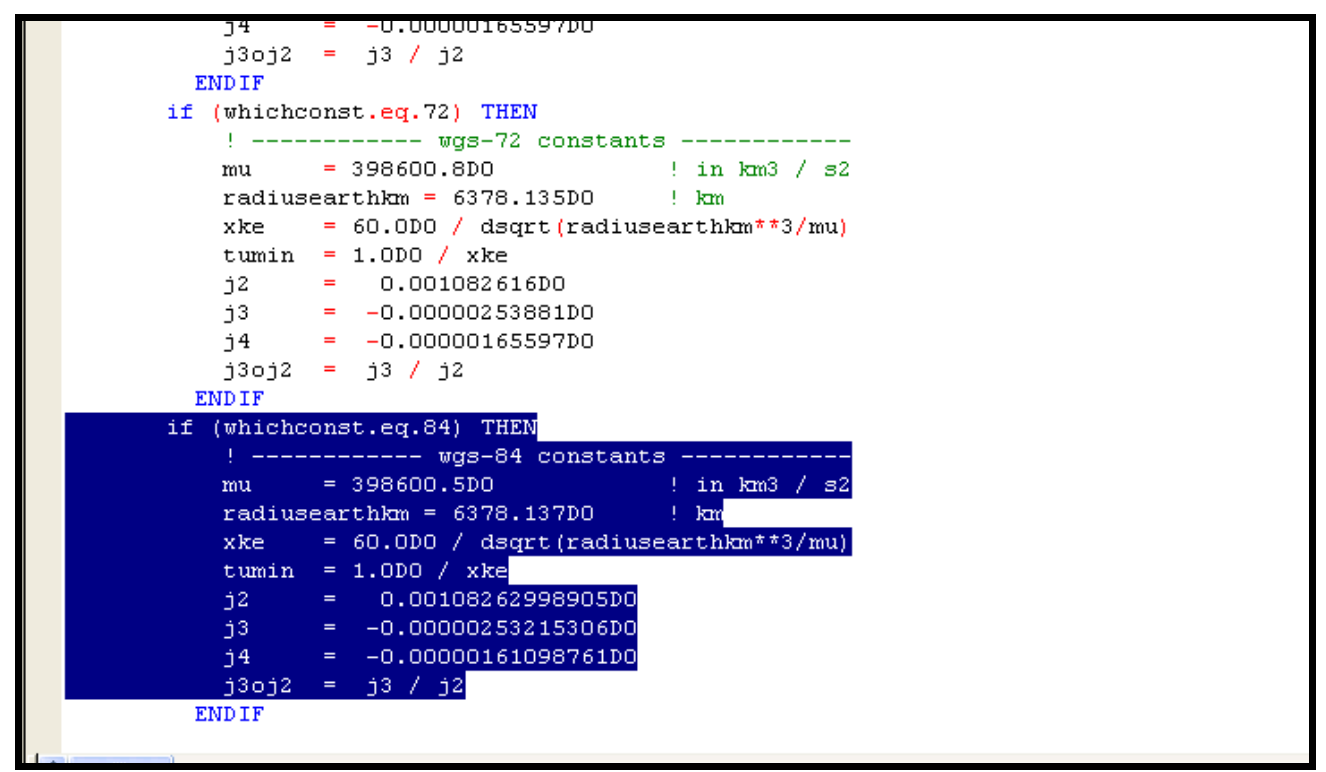

Figure 69 - Changing WGS values (2)

\subsubsection{B*}

The $\mathrm{B}^{*}$ term is used to estimate the drag force felt by the object in orbit. For the Orbital Debris Program Office, this value has been set to zero since B* is not calculated by the PREDICT program and the objects in GEO do not experience significant drag forces. There are two ways to change the default value of $\mathrm{B}^{*}$. First, the user can define it as something other than zero inside the program. To do this, find where $\mathrm{B}^{*}$ is defined in the TwoLine2RVSGP4 subroutine ( line 1157$)$. Change the $0.0 \mathrm{~d} 0$ value to desired $\mathrm{B}^{*}$ value. Second, the user can comment the above line out completely and uncomment the two lines above. Doing this will prompt the user to input a B* term. This may cause some problems in running the PREDICT code since it will prompt the user during every 
iteration of the SGP4 program. To solve this, the user can comment out all three lines and define the $\mathrm{B}^{*}$ term in the main program. The term can then be accessed by the subroutine either by a global variable or by having the $\mathrm{B}^{*}$ variable be called by the subroutine itself.

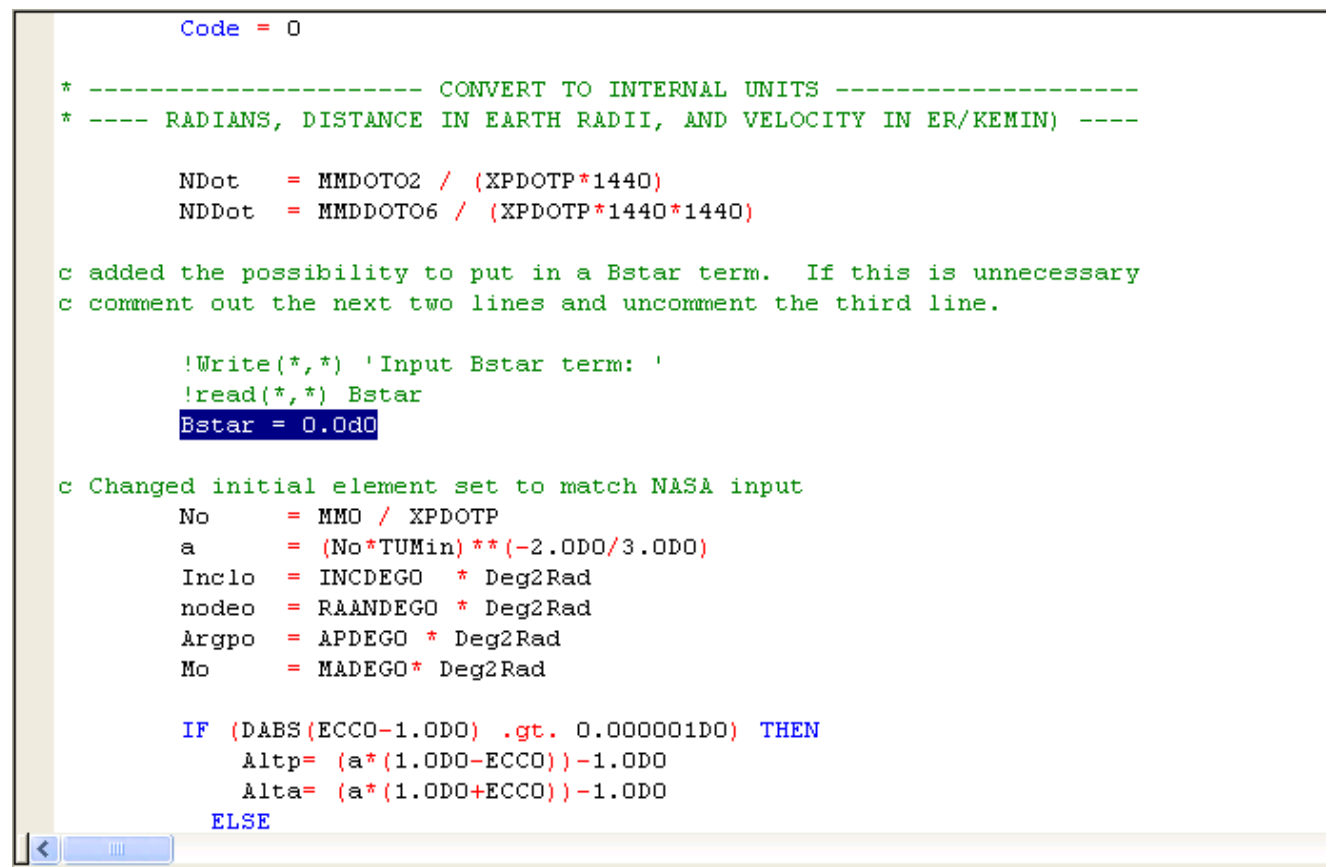

Figure 70 - Changing the $B *$ term 


\section{BIBLIOGRAPHY}

1. AIAA-2006-6753.zip 700 KB, Feb 16, 2009. ,

$<$ http://www.centerforspace.com/downloads/>

2. Chobotov, Vladimir A. Orbital Mechanics, Second Edition. Reston, VA, AIAA, Inc., 1996.

3. Hoots, Felix R., et al. "History of Analytical Orbit Modeling in the U.S. Space

Surveillance System.” Journal of Guidance Control, and Dynamics March-April 2004: 174-185.

4. Hoots, Felix R. and Roehrich, Ronal L. "Models for Propagation of NORAD

Element Sets.” SPACETRACK REPORT NO.3 December 1980: Packaged compiled by Kelso, T.S.: December 31, 1988. <

ftp://ftp.amsat.org/amsat/docs/spacetrk.pdf>

5. Kelso, T.S. “TLE Data”, 2008-2009. < http://celestrak.com/>

6. Meissner, Loren P. Fortran 90. Boston, MA, PWS Publishing Co., 1995

7. "Selected Orbital Data" 2009. <http://www.heavens-above.com> 
8. Vallado, David A. Fundamentals of Astrodynamics and Applications, Third Edition. Hawthorne CA, Microcosm Press, 2007.

9. Vallado, David A., et al. "Revisiting Spacetrack Report \#3.” 2006.

$<$ http://celestrak.com/publications/AIAA/2006-6753/AIAA-2006-6753.pdf $>$

10. “World Geodetic System.” Wikipedia May 11, 2009.

$<$ http://en.wikipedia.org/wiki/WGS84> 


\section{APPENDIX A. SGP4 Code}

All working codes are included in the accompanying CD. The FORTRAN code designed to replace the legacy SGP4 propagator within PREDICT is displayed below for reference.

\section{A.1 Modified FORTRAN Code (Vallado)}

This code compiles and runs on LAHEY express 7.2. Output files are generated;

however, when testing the code with PREDICT_Feb09, there were issues with rewriting a file at the end of the program. There is no evidence that this is linked with the new

SGP4 propagator.

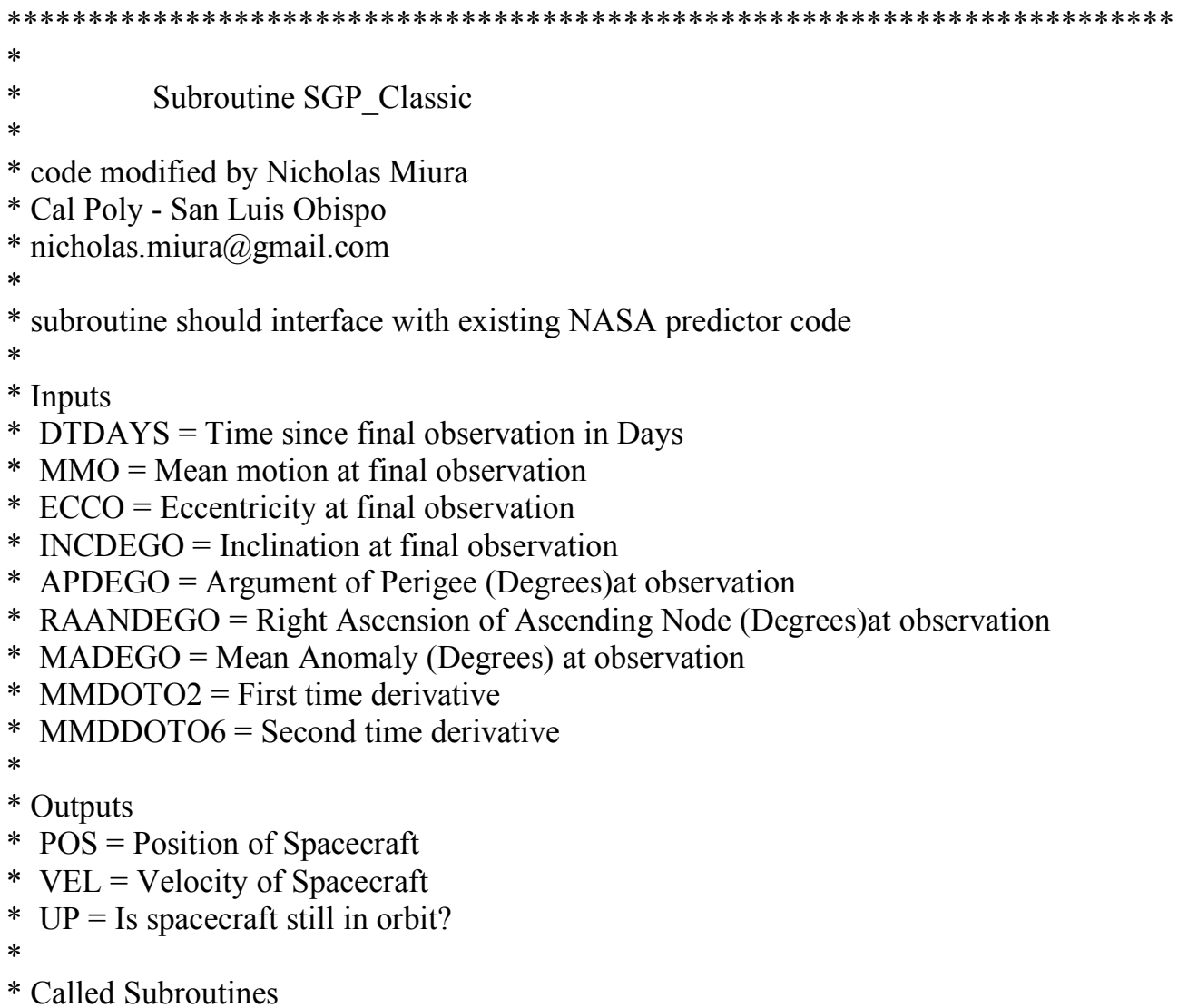


* getgravconst

Subroutine SGP_Classic(DTDAYS,MM0,ECC0, \& INCDEG0,APDEG0,RAANDEG0,MADEG0, \& MMDOTO2,MMDDOTO6,POS,VEL,UP)

\section{IMPLICIT NONE}

$c$ these declarations are copied from NASA Predictor code $\mathrm{c}$ and define the input and output of the subroutine

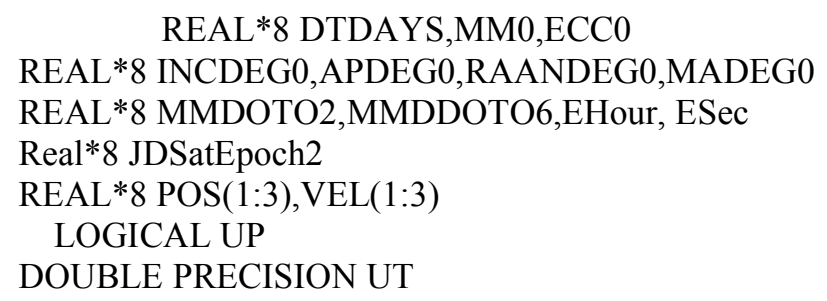

$\mathrm{c}$ these declarations are used in the code

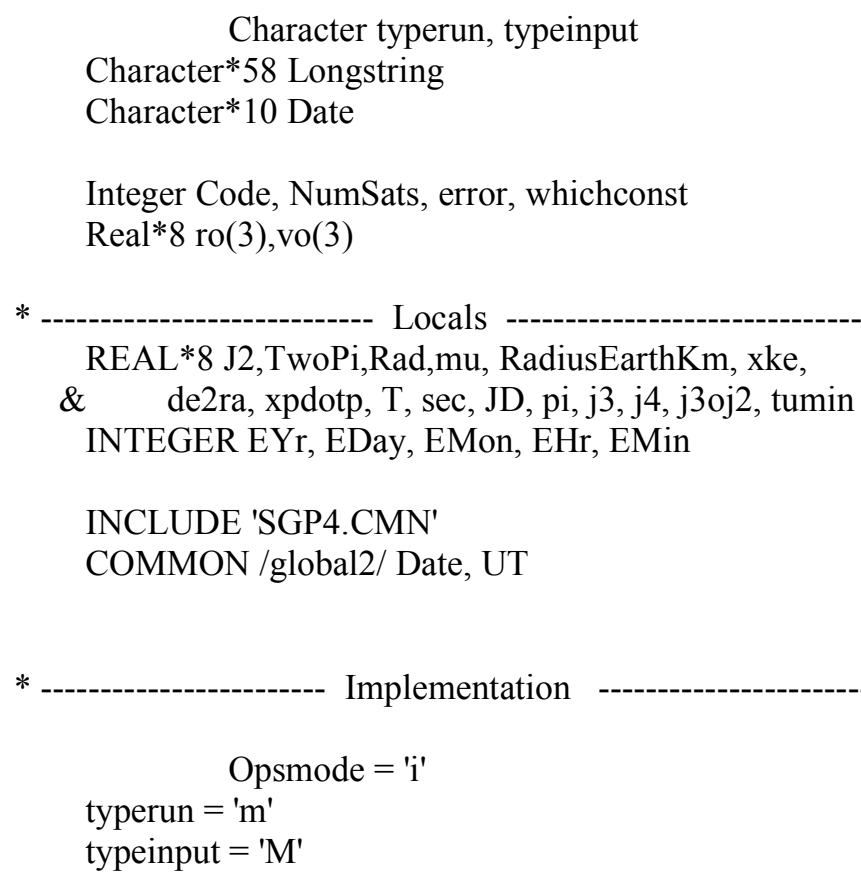




$$
\begin{aligned}
& \text { whichconst }=72 \\
& \text { pi }=4.0 \mathrm{D} 0 * \text { datan }(1.0 \mathrm{D} 0) ! 3.14159265358979 \mathrm{D} 0 \\
& \text { TwoPi }=2.0 \mathrm{D} 0 * \mathrm{pi} \quad ! 6.28318530717959 \mathrm{D} 0 \\
& \mathrm{Rad}=180.0 \mathrm{D} 0 / \mathrm{pi} \quad ! 57.29577951308230 \mathrm{D} 0 \\
& \begin{array}{l}
\mathrm{DE} 2 \mathrm{RA} \\
\text { xpdotp }=1440.0 /(2.0 * \mathrm{pi}) ! 229.1831180523293 \mathrm{D} 0
\end{array}
\end{aligned}
$$

c sgp4fix identify constants and allow alternate values

CALL getgravconst( whichconst, tumin, mu, radiusearthkm, xke,

$$
\text { \& } \quad \mathrm{j} 2, \mathrm{j} 3, \mathrm{j} 4, \mathrm{j} 3 \mathrm{oj} 2 \text { ) }
$$

c Read Date and Time

$$
\begin{aligned}
& \text { READ (Date(1:4),FMT='(I4)') EYr } \\
& \text { READ (Date(6:7),FMT='(I2)') EMon } \\
& \text { READ (Date(9:10),FMT='(I2)') EDay }
\end{aligned}
$$

CALL HMS (UT, EHr, EMin, ESec)

CALL JDAY1 (EYr, EMon, EDay, EHr ,EMin, ESec, JDSatEpoch2)

c Open a temporary file containing initialized orbital data

c Called by other subroutines

c OPEN(115,FILE = 'Sgp4Rec.bak', ACCESS = 'DIRECT',

c \& FORM $=$ 'UNFORMATTED', RECL $=1100$, STATUS $=$ 'REPLACE' $)$

$$
\text { NumSats }=1
$$

c bring orbital elements to the new two line element converter - trick the system
CALL TwoLine2RVSGP4 ( NumSats,typerun,typeinput,whichconst,
$\& \quad$ Code, DTDAYS,MM0,ECC0,
\& INCDEG0,APDEG0,RAANDEG0,MADEG0,
\& MMDOTO2,MMDDOTO6, JDSatEpoch2, Error )

c Call the SGP4 propagator based on Time in minutes

$$
\begin{aligned}
& \text { T = DTDAYS* } 1440 \\
& \text { CALL SGP4 ( whichconst, T, Ro, Vo, Error ) } \\
& \text { If (Error .eq. 1)Then } \\
& \text { Up = .FALSE. }
\end{aligned}
$$




\section{ENDIF}

$\mathrm{c}$ the next WRITE line displays the result on the screen

c comment this out if you don't want to test the results

c WRITE( *,800 ) T, ro(1),ro(2),ro(3),vo(1),vo(2),vo(3)

c 800 FORMAT(F17.8,3F17.8,3(1X,F14.9))

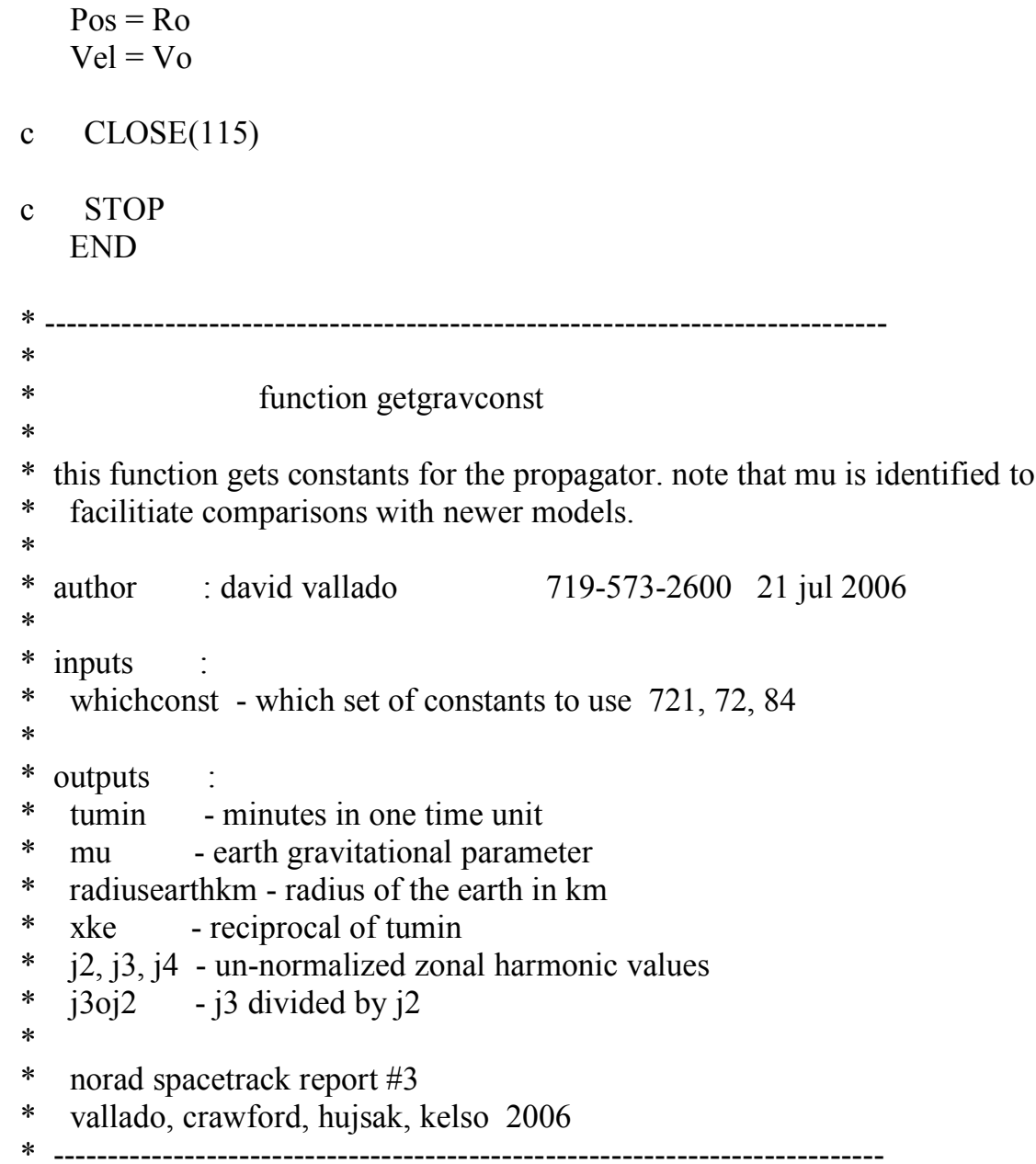

SUBROUTINE getgravconst ( whichconst, tumin, mu, \& radiusearthkm, xke, j2, j3, j4, j3oj2 )

IMPLICIT NONE 
REAL*8 radiusearthkm, xke, j2, j3, j4, j3oj2, mu, tumin

INTEGER whichconst

if (whichconst.eq.721) THEN

! -- wgs-72 low precision str\#3 constants --

radiusearthkm $=6378.135 \mathrm{D} 0 \quad ! \mathrm{km}$

xke $=0.0743669161 \mathrm{D} 0$

$\mathrm{mu}=398600.79964 \mathrm{D} 0 \quad$ ! in $\mathrm{km} 3 / \mathrm{s} 2$

tumin $=1.0 \mathrm{D} 0 / \mathrm{xke}$

$\mathrm{j} 2=0.001082616 \mathrm{D} 0$

$\mathrm{j} 3=-0.00000253881 \mathrm{D} 0$

$\mathrm{j} 4=-0.00000165597 \mathrm{D} 0$

$\mathrm{j} 3 \mathrm{oj} 2=\mathrm{j} 3 / \mathrm{j} 2$

ENDIF

if (whichconst.eq.72) THEN

! -------- wgs-72 constants

$\mathrm{mu}=398600.8 \mathrm{D} 0 \quad$ ! in $\mathrm{km} 3 / \mathrm{s} 2$

radiusearthkm $=6378.135 \mathrm{D} 0 \quad ! \mathrm{km}$

xke $=60.0 \mathrm{D} 0 / \mathrm{dsqrt}($ radiusearthkm $* * 3 / \mathrm{mu})$

tumin $=1.0 \mathrm{D} 0 / \mathrm{xke}$

$\mathrm{j} 2=0.001082616 \mathrm{D} 0$

$\mathrm{j} 3=-0.00000253881 \mathrm{D} 0$

$\mathrm{j} 4=-0.00000165597 \mathrm{D} 0$

$\mathrm{j} 3 \mathrm{oj} 2=\mathrm{j} 3 / \mathrm{j} 2$

ENDIF

if (whichconst.eq.84) THEN

! ----------- wgs-84 constants ------------

$\mathrm{mu}=398600.5 \mathrm{D} 0 \quad$ ! in $\mathrm{km} 3 / \mathrm{s} 2$

radiusearthkm $=6378.137 \mathrm{D} 0 \quad ! \mathrm{km}$

xke $=60.0 \mathrm{D} 0 / \mathrm{dsqrt}($ radiusearthkm $* * 3 / \mathrm{mu})$

tumin $=1.0 \mathrm{D} 0 / \mathrm{xke}$

$\mathrm{j} 2=0.00108262998905 \mathrm{D} 0$

$\mathrm{j} 3=-0.00000253215306 \mathrm{D} 0$

$\mathrm{j} 4=-0.00000161098761 \mathrm{D} 0$

j3oj2 $=\mathrm{j} 3 / \mathrm{j} 2$

ENDIF

RETURN

END ! SUBROUTINE getgravconst

* this function converts the two line element set character string data to

* variables and initializes the sgp4 variables. several intermediate variables 
* and quantities are determined. note that the result is a "structure" so multiple

* satellites can be processed simultaneously without having to reinitialize. the

* verification mode is an important option that permits quick checks of any

* changes to the underlying technical theory. this option works using a

* modified TLE file in which the start, stop, and delta time values are

* included at the end of the second line of data. this only works with the

* verification mode. the catalog mode simply propagates from -1440 to $1440 \mathrm{~min}$

* from epoch and is useful when performing entire catalog runs.

* author : david vallado $\quad 719-573-2600 \quad 1$ mar 2001

$*$

* inputs :

* Numsats - Number of satellites processed. It also becomes the record

* number for each satellite

* typerun - type of run verification ' $\mathrm{V}$ ', catalog 'C',

* manual ' $\mathrm{M}$ '

* typeinput - type of manual input mfe 'M', epoch 'E', dayofyr 'D'

* whichconst - which set of constants to use 72,84

* opsmode - type of manual input afspc 'a', imporved ' $i$ '

$*$

* outputs :

* Code - EOF indicator. Code $=999$ when EOF reached

* startmfe - starttime of simulation, min from epoch

* stopmfe - stoptime of simulation, min from epoch

* deltamin - time step min

* coupling :

* days2mdhms - conversion of days to month, day, hour, minute, second

* jday - convert day month year hour minute second into Julian date

* sgp4init - initialize the sgp4 variables

* Files :

* Unit 10 - test.elm input 2-line element set file

* Unit 11 - test.bak output file

* Unit 15 - sgp4rec.bak temporary file of record for 2 line element sets

$*$

* references :

* norad spacetrack report \#3

* vallado, crawford, hujsak, kelso 2006

SUBROUTINE TwoLine2RVSGP4 ( NumSats, Typerun, typeinput, $\&$

$\&$ whichconst, Code, DTDAYS,MM0,ECC0, INCDEG0,APDEG0,RAANDEG0,MADEG0, \& MMDOTO2,MMDDOTO6, JDSatEpoch1, Error ) 
IMPLICIT NONE

Character Typerun, typeinput

Integer Code, NumSats, whichconst

REAL $* 8$ startmfe, stopmfe, deltamin

* -------------------------- Locals

REAL*8 J2, mu, RadiusEarthKm,VKmPerSec, xke, tumin

REAL*8 BC,EPDay, sec, xpdotp, j3, j4, j3oj2

REAL $* 8$ startsec, stopsec, startdayofyr, stopdayofyr, jdstart,

\& jdstop, JDSatEpoch 1

INTEGER startyear, stopyear, startmon, stopmon, startday,

\& stopday, starthr, stophr, startmin, stopmin

INTEGER Yr,Mon,Day,Hr,Minute, ICrdno,nexp,bexp, error

CHARACTER Show

Character*130 LongStr1,LongStr2

REAL*8 DTDAYS,MM0,ECC0

REAL*8 INCDEG0,APDEG0,RAANDEG0,MADEG0

REAL*8 MMDOTO2,MMDDOTO6

COMMON /DebugHelp/ Help

CHARACTER Help

INCLUDE 'SGP4.CMN'

INCLUDE 'ASTMATH.CMN'

! -------------------- Implementation -----------------------

Show $=$ 'N'

xpdotp $=1440.0 \mathrm{D} 0 /(2.0 \mathrm{D} 0 *$ pi $) ! 229.1831180523293$

CALL getgravconst( whichconst, tumin, mu, radiusearthkm, xke, \& $\quad \mathrm{j} 2, \mathrm{j} 3, \mathrm{j} 4, \mathrm{j} 3 \mathrm{oj} 2)$;

VKmPerSec $=$ RadiusEarthKm * xke / 60.0D0

* -------------- READ THE FIRST LINE OF ELEMENT SET

Code $=0$

* --_---- CONVERT TO INTERNAL UNITS

* ---- RADIANS, DISTANCE IN EARTH RADII, AND VELOCITY IN ER/KEMIN) -$--$

NDot $=$ MMDOTO2 / (XPDOTP*1440)

NDDot $=$ MMDDOTO6 $/($ XPDOTP*1440*1440) 
c added the possibility to put in a Bstar term. If this is unnecessary

c comment out the next two lines and uncomment the third line.

!Write $(*, *)$ 'Input Bstar term: '

!read $(*, *)$ Bstar

Bstar $=0.0 \mathrm{~d} 0$

c Changed initial element set to match NASA input

No $=$ MM0 / XPDOTP

$\mathrm{a}=(\mathrm{No} * \mathrm{TUMin}) * *(-2.0 \mathrm{D} 0 / 3.0 \mathrm{D} 0)$

Inclo $=$ INCDEG0 $*$ Deg2Rad

nodeo $=$ RAANDEG0 $*$ Deg2Rad

Argpo $=$ APDEG0 $*$ Deg2Rad

Mo $=$ MADEG0 $*$ Deg2Rad

IF (DABS(ECC0-1.0D0) .gt. 0.000001D0) THEN

$\mathrm{Altp}=\left(\mathrm{a}^{*}(1.0 \mathrm{D} 0-\mathrm{ECC} 0)\right)-1.0 \mathrm{D} 0$

Alta $=(a *(1.0 \mathrm{D} 0+\mathrm{ECC} 0))-1.0 \mathrm{D} 0$

ELSE

Alta $=999999.9 \mathrm{D} 0$

Altp $=2.0 \mathrm{D} 0 *(4.0 \mathrm{D} 0 /(\mathrm{No} * \mathrm{No}) * *(1.0 \mathrm{D} 0 / 3.0 \mathrm{D} 0))$

ENDIF

! ---- Ballistic Coefficient ----

IF (DABS(BStar) .gt. 0.00000001D0) THEN

$\mathrm{BC}=1.0 \mathrm{D} 0 /(12.741621 \mathrm{D} 0 * \mathrm{BStar})$

ELSE

$\mathrm{BC}=1.111111111111111 \mathrm{D} 0$

ENDIF

$!$

! find sgp4epoch time of element set

! remember that sgp4 uses units of days from 0 jan 1950 (sgp4epoch)

! and minutes from the epoch (time)

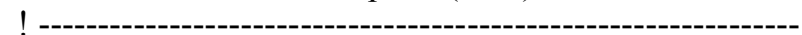

* ----------- MAKE INITIAL PREDICTION AT EPOCH -------------

! $2433281.5-2400000.5=33281.0$, thus time from 1950

CALL SGP4Init( whichconst,

$\& \quad$ SatNum,BStar, ECC0, JDSatEpoch1-2433281.5D0,

\& Argpo,Inclo,Mo,No, nodeo, Error )

Formatted: German

IF(Error .GT. 0) THEN

WRITE( **) '\#*** SGP4 Model Error ***',Error

ENDIF 
c write tle output details

c INCLUDE 'debug8.for'

!---- Fix to indicate end-of-file

GOTO 1000

999 Code $=999$

1000 CONTINUE

RETURN

END！ SUBROUTINE TwoLine2RVSGP4

$*$

$*$

$*$

$*$

* This subroutine initializes variables for SGP4.

*

* author : david vallado 719-573-2600 28 jun 2005

*

* inputs :

* satn - satellite number

* bstar - sgp4 type drag coefficient $\quad \mathrm{kg} / \mathrm{m} 2 \mathrm{er}$

* ecco - eccentricity

* epoch - epoch time in days from jan $0,1950.0 \mathrm{hr}$

* argpo - argument of perigee (output if ds)

* inclo - inclination

* mo - mean anomaly (output if ds)

* no - mean motion

* nodeo - right ascension of ascending node

$*$

* outputs :

* satrec - common block values for subsequent calls

* return code - non-zero on error.

* $\quad 1-$ mean elements, ecc $>=1.0$ or ecc $<-0.001$ or a $<0.95$ er

* $\quad 2$ - mean motion less than 0.0

* $\quad 3$ - pert elements, ecc $<0.0$ or ecc $>1.0$

* $\quad 4$ - semi-latus rectum $<0.0$

* $\quad 5$ - epoch elements are sub-orbital

* 6 - satellite has decayed

* coupling :

* getgravconst-

* initl

* dscom

* dpper - 
c sgp4fix - note the following variables are also passed directly via sgp4 common.

c it is possible to streamline the sgp4init call by deleting the " $\mathrm{x"}$

c variables, but the user would need to set the common values first. we

c include the additional assignment in case twoline $2 \mathrm{rv}$ is not used.

$$
\begin{aligned}
& \text { bstar }=\text { xbstar } \\
& \text { ecco }=\text { xecco } \\
& \text { argpo }=\text { xargpo } \\
& \text { inclo }=\text { xinclo } \\
& \text { mo }=\text { xmo } \\
& \text { no }=\text { xno } \\
& \text { nodeo }=\text { xnodeo }
\end{aligned}
$$

! sgp4fix identify constants and allow alternate values

CALL getgravconst( whichconst, tumin, mu, radiusearthkm, xke,

$\&$

$$
\mathrm{j} 2, \mathrm{j} 3, \mathrm{j} 4, \mathrm{j} 3 \mathrm{oj} 2 \text { ) }
$$

$\mathrm{SS}=78.0 \mathrm{D} 0 /$ RadiusEarthKm $+1.0 \mathrm{D} 0$

QZMS2T $=((120.0 \mathrm{D} 0-78.0 \mathrm{D} 0) /$ RadiusEarthKm $) * * 4$

$\mathrm{X} 2 \mathrm{o} 3=2.0 \mathrm{D} 0 / 3.0 \mathrm{D} 0$

c sgp4fix divisor for divide by zero check on inclination

c the old check used 1.0D0 + $\cos ($ pi-1.0D-9), but then compared it to

c $1.5 \mathrm{D}-12$, so the threshold was changed to $1.5 \mathrm{D}-12$ for consistency

temp $4=1.5 \mathrm{D}-12$

Init $=$ ' $y$ '

$\mathrm{T}=0.0 \mathrm{D} 0$

CALL INITL( Satn, whichconst, Ecco , EPOCH , Inclo, No,

\& Method, AINV , AO , CON41, CON42, COSIO, COSIO2,

\& Eccsq, OMEOSQ, POSQ , rp , RTEOSQ, SINIO,

\& GSTo, Opsmode )

IF(rp .lt. 1.0D0) THEN

Error $=5$

ENDIF

IF(OMEOSQ .ge. 0.0D0 .OR. No .ge. 0.0D0) THEN

ISIMP $=0$

IF (rp .lt. (220.0D0/RadiusEarthKm+1.0D0)) THEN

ISIMP $=1$

ENDIF

$\mathrm{SFour}=\mathrm{SS}$

QZMS24 = QZMS2T

Formatted: French

(France) 


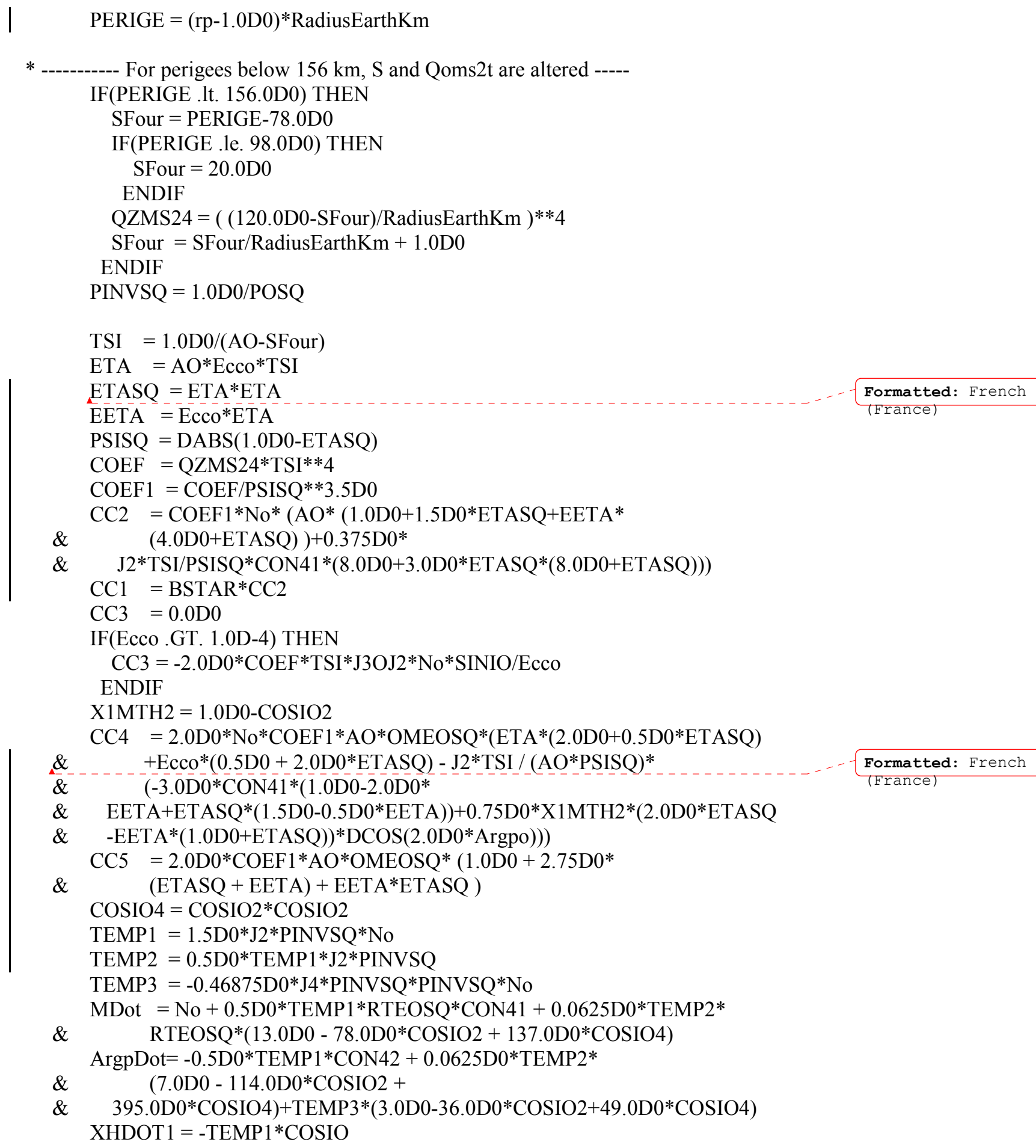




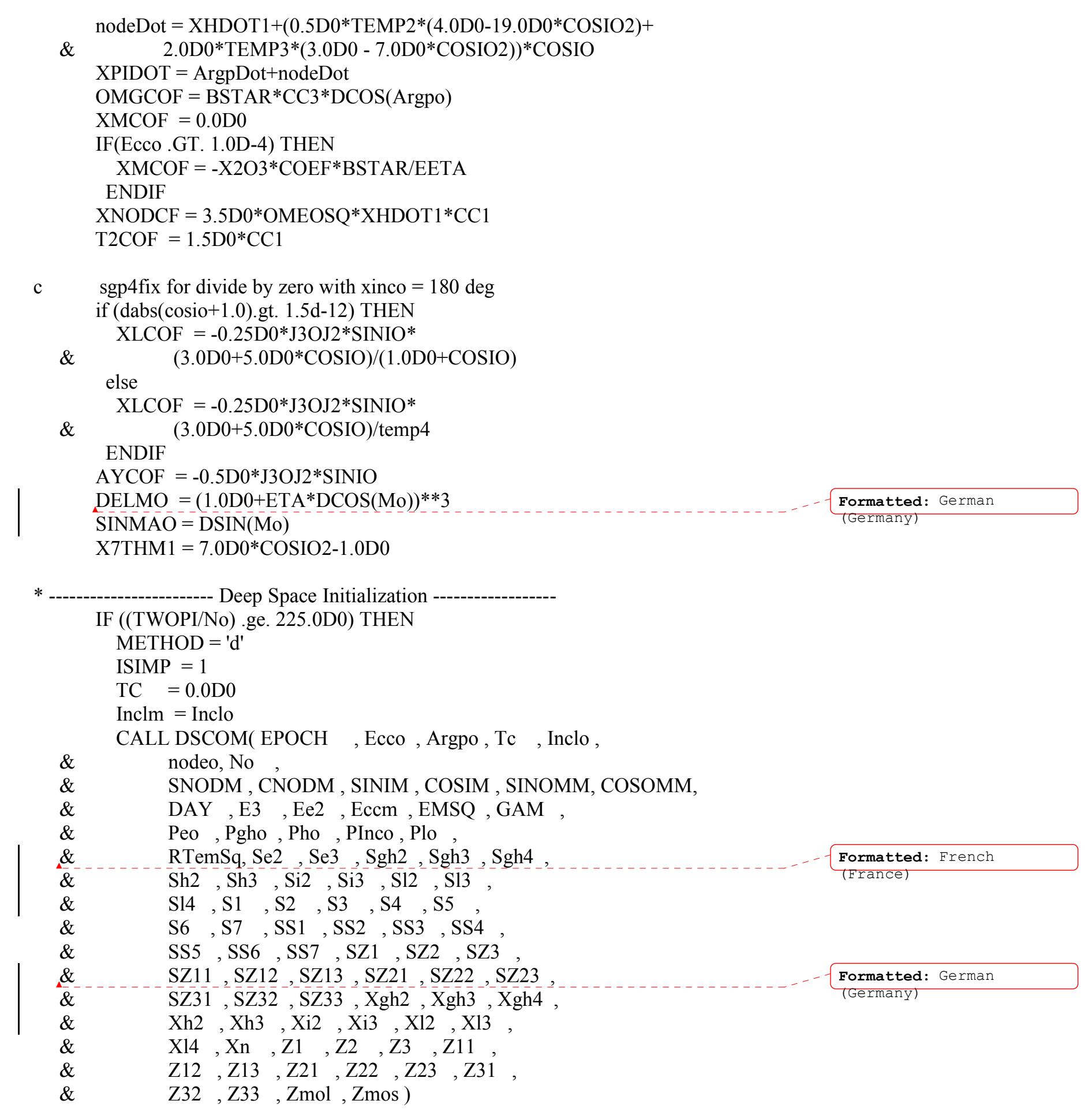


CALL DPPER( e3, ee2, peo , pgho, pho , pinco,

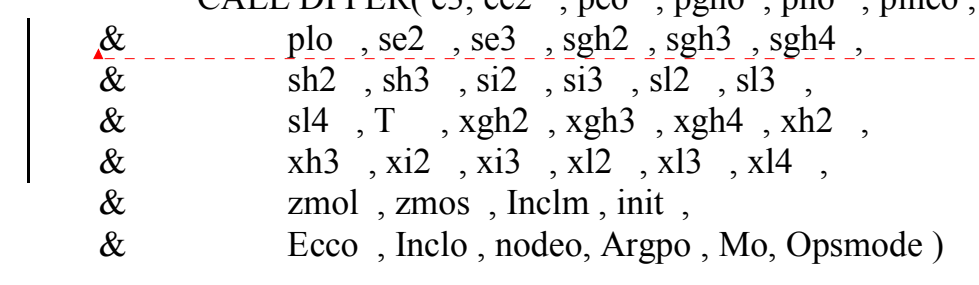

Argpm $=0.0 \mathrm{D} 0 !$ add for DS to work initial

nodem $=0.0 \mathrm{D} 0$

$\mathrm{Mm}=0.0 \mathrm{D} 0$

CALL DSINIT( whichconst,

\& $\quad$ Cosim ,Emsq, Argpo, S1 , S2 , S3 ,

\& $\quad \mathrm{S} 4, \mathrm{~S} 5$, , Sinim, Ss1 , Ss2 , Ss3 ,

\& $\quad$ Ss4 , Ss5 , Sz1 , Sz3 , Sz11 , Sz13 ,

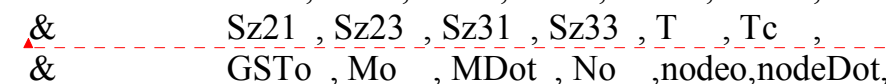

Formatted: German

\& $\quad$ XPIDOT, Z1 , Z3 , Z11 , Z13 , Z21 ,

(Germany)

\& Z23, Z31 , Z33 , ecco , eccsq,

\& Eccm, Argpm, Inclm, Mm , Xn , nodem,

\& $\quad$ IREZ , Atime, D2201, D2211, D3210, D3222,

$\&$ D4410, D4422, D5220, D5232, D5421, D5433,

$\&$ Dedt , Didt , DMDT , DNDT , DNODT, DOMDT,

$\&$ Del1, Del2, Del3 , Xfact, Xlamo, Xli

\&

ENDIF

* ------- Set variables if not deep space or $\mathrm{rp}<220$

IF (ISIMP .ne. 1) THEN

$\mathrm{CC} 1 \mathrm{SQ}=\mathrm{CC} 1 * \mathrm{CC} 1$

$\mathrm{D} 2=4.0 \mathrm{D} 0 * \mathrm{AO} * \mathrm{TSI} * \mathrm{CC} 1 \mathrm{SQ}$

$\mathrm{TEMP}=\mathrm{D} 2 * \mathrm{TSI} * \mathrm{CC} 1 / 3.0 \mathrm{D} 0$

D3 $=(17.0 \mathrm{D} 0 * \mathrm{AO}+$ SFour $) *$ TEMP

$\mathrm{D} 4=0.5 \mathrm{D} 0 * \mathrm{TEMP} * \mathrm{AO} * \mathrm{TSI} *$

\& $\quad(221.0 \mathrm{D} 0 * \mathrm{AO}+31.0 \mathrm{D} 0 * \mathrm{SFour}) * \mathrm{CC} 1$

$\mathrm{T} 3 \mathrm{COF}=\mathrm{D} 2+2.0 \mathrm{D} 0 * \mathrm{C} \overline{\mathrm{C}} \mathrm{SQ}$

Formatted: French

$\mathrm{T} 4 \mathrm{COF}=0.25 \mathrm{D} 0 *(3.0 \mathrm{D} 0 * \mathrm{D} 3+\mathrm{CC} 1 *(12.0 \mathrm{D} 0 * \mathrm{D} 2+10.0 \mathrm{D} 0 * \mathrm{CC} 1 \mathrm{SQ}))$

$\mathrm{T} 5 \mathrm{COF}=0.2 \mathrm{D} 0 *(3.0 \mathrm{D} 0 * \mathrm{D} 4+12.0 \mathrm{D} 0 * \mathrm{CC} 1 * \mathrm{D} 3+6.0 \mathrm{D} 0 * \mathrm{D} 2 * \mathrm{D} 2+$

\& $\left.\quad 15.0 \mathrm{D} 0 * \mathrm{CC} 1 \mathrm{SQ}^{*}(2.0 \mathrm{D} 0 * \mathrm{D} 2+\mathrm{CC} 1 \mathrm{SQ})\right)$

ENDIF

ENDIF ! ------ if nodeo and No are gtr 0

init $=$ ' $n$ ' 
CALL SGP4(whichconst, 0.0D0, r, v, error)

RETURN

END ! end sgp4init

$*$

$*$

* this procedure is the sgp4 prediction model from space command. this is an

* updated and combined version of sgp4 and sdp4, which were originally

* published separately in spacetrack report \#3. this version follows the

* methodology from the aiaa paper (2006) describing the history and

* development of the code.

$*$

* author : david vallado 719-573-2600 28 jun 2005

*

* inputs :

* satrec - initialised structure from sgp4init() call.

* tsince - time eince epoch (minutes)

$*$

* outputs :

* $\mathrm{r} \quad$ - position vector $\quad \mathrm{km}$

* v - velocity $\mathrm{km} / \mathrm{sec}$

$*$

* return code - non-zero on error.

* $\quad 1-$ mean elements, ecc $>=1.0$ or ecc $<-0.001$ or a $<0.95$ er

* $\quad 2$ - mean motion less than 0.0

* $\quad 3$ - pert elements, ecc $<0.0$ or ecc $>1.0$

* $\quad 4$ - semi-latus rectum $<0.0$

* $\quad 5$ - epoch elements are sub-orbital

* 6 - satellite has decayed

$*$

$*$

* coupling :

* getgravconst-

* dpper

* dpspace

*

* references :

* hoots, roehrich, norad spacetrack report \#3 1980

* hoots, norad spacetrack report \#6 1986

* hoots, schumacher and glover 2004

* vallado, crawford, hujsak, kelso 2006 
SUBROUTINE SGP4 ( whichconst, T, r, v, Error )

IMPLICIT NONE

INTEGER Error, whichconst

REAL*8 T, r(3), v(3)

INCLUDE 'SGP4.CMN'$$
\text { Local Variables }
$$

REAL*8 AM , Axnl , Aynl , Betal, COSIM, Cnod ,

\& Cos2u, Coseo1, Cosi , Cosip, Cosisq, Cossu, Cosu ,

\& Delm , Delomg, Eccm , EMSQ , Ecose, E12 , Eo1 ,

\& Eccp , Esine, Argpm, Argpp , Omgadf, Pl ,

\& Rdotl, R1 , Rvdot, Rvdotl, SINIM ,

$\& \quad$ Sin2u, Sineo 1, Sini , Sinip, Sinsu, Sinu ,

$\& \quad$ Snod $, \mathrm{Su}, \mathrm{T} 2, \mathrm{~T} 3, \mathrm{~T} 4, \mathrm{Tem} 5, \mathrm{Temp}$

\& Temp1, Temp2, Tempa, Tempe, Templ, U , Ux ,

\& $\quad \mathrm{Uy}, \mathrm{Uz}, \mathrm{Vx}, \mathrm{Vy}, \mathrm{Vz}, \mathrm{Inclm}, \mathrm{Mm}$,

\& $\quad \mathrm{XN}$, nodem, Xinc , Xincp, Xl , Xlm , Mp ,

\& Xmdf , Xmx , Xmy , Xnoddf, Xnode, nodep,

$\& \quad$ Tc , Dndt

REAL*8 X2O3, J2,J3,XKE,J3OJ2, mr,mv,

\& mu, RadiusEarthkm, VKmPerSec, temp4, tumin, j4 INTEGER iter

Formatted: German

(Germany)

CHARACTER Help

INCLUDE 'ASTMATH.CMN'

* -

* ---------------- SET MATHEMATICAL CONSTANTS

$\mathrm{X} 2 \mathrm{O} 3=2.0 \mathrm{D} 0 / 3.0 \mathrm{D} 0$

c Keep compiler ok for warnings on uninitialized variables

$\mathrm{mr}=0.0 \mathrm{D} 0$

Coseo $1=1.0 \mathrm{D} 0$

Sineo $1=0.0 \mathrm{D} 0$

! sgp4fix identify constants and allow alternate values

CALL getgravconst( whichconst, tumin, mu, radiusearthkm, xke,

$\& \quad \mathrm{j} 2, \mathrm{j} 3, \mathrm{j} 4, \mathrm{j} 3 \mathrm{oj} 2)$

c sgp4fix divisor for divide by zero check on inclination

c the old check used 1.0D0 + $\cos ($ pi-1.0D-9), but then compared it to

c $1.5 \mathrm{D}-12$, so the threshold was changed to $1.5 \mathrm{D}-12$ for consistency

temp4 $=1.5 \mathrm{D}-12$ 


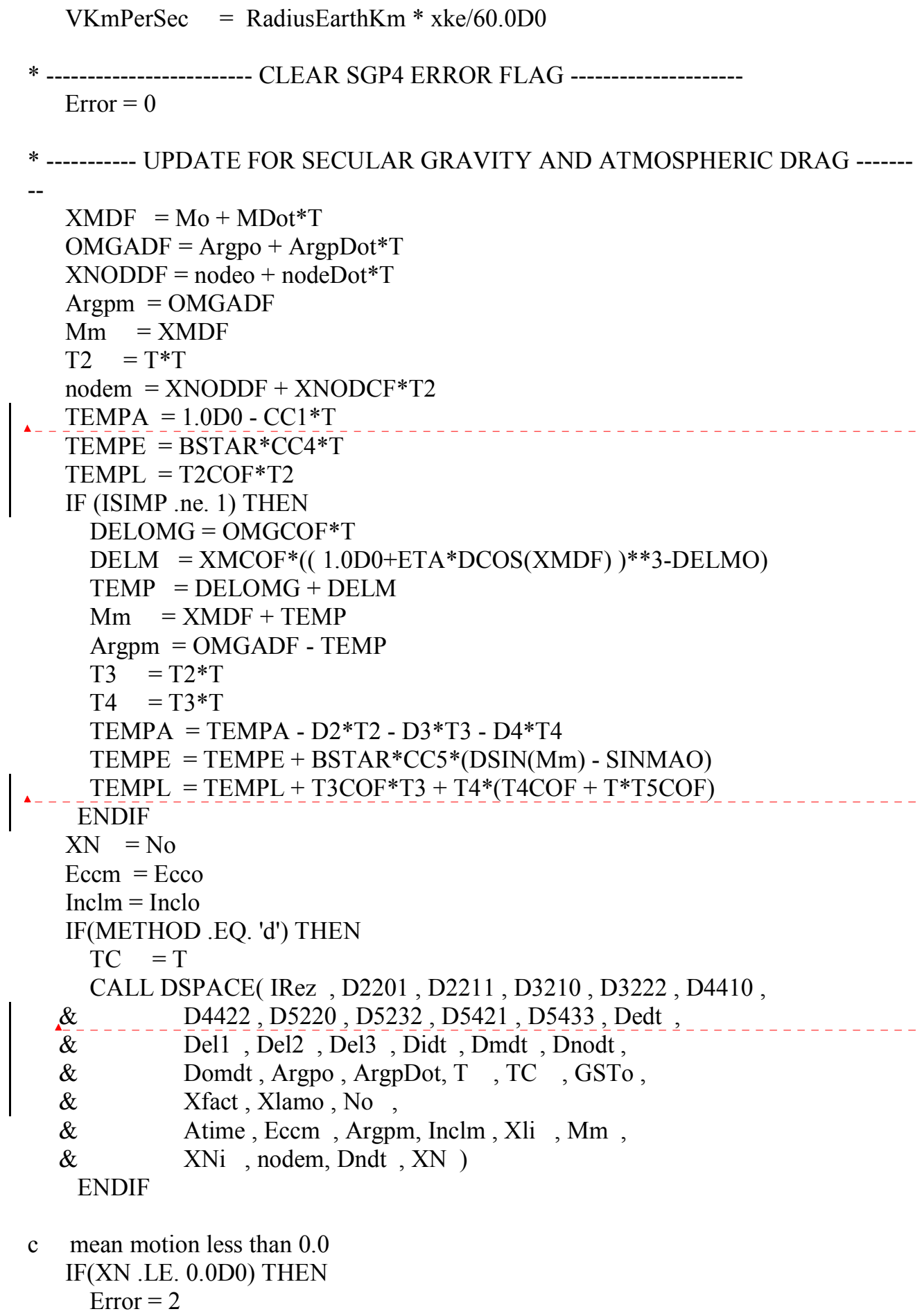




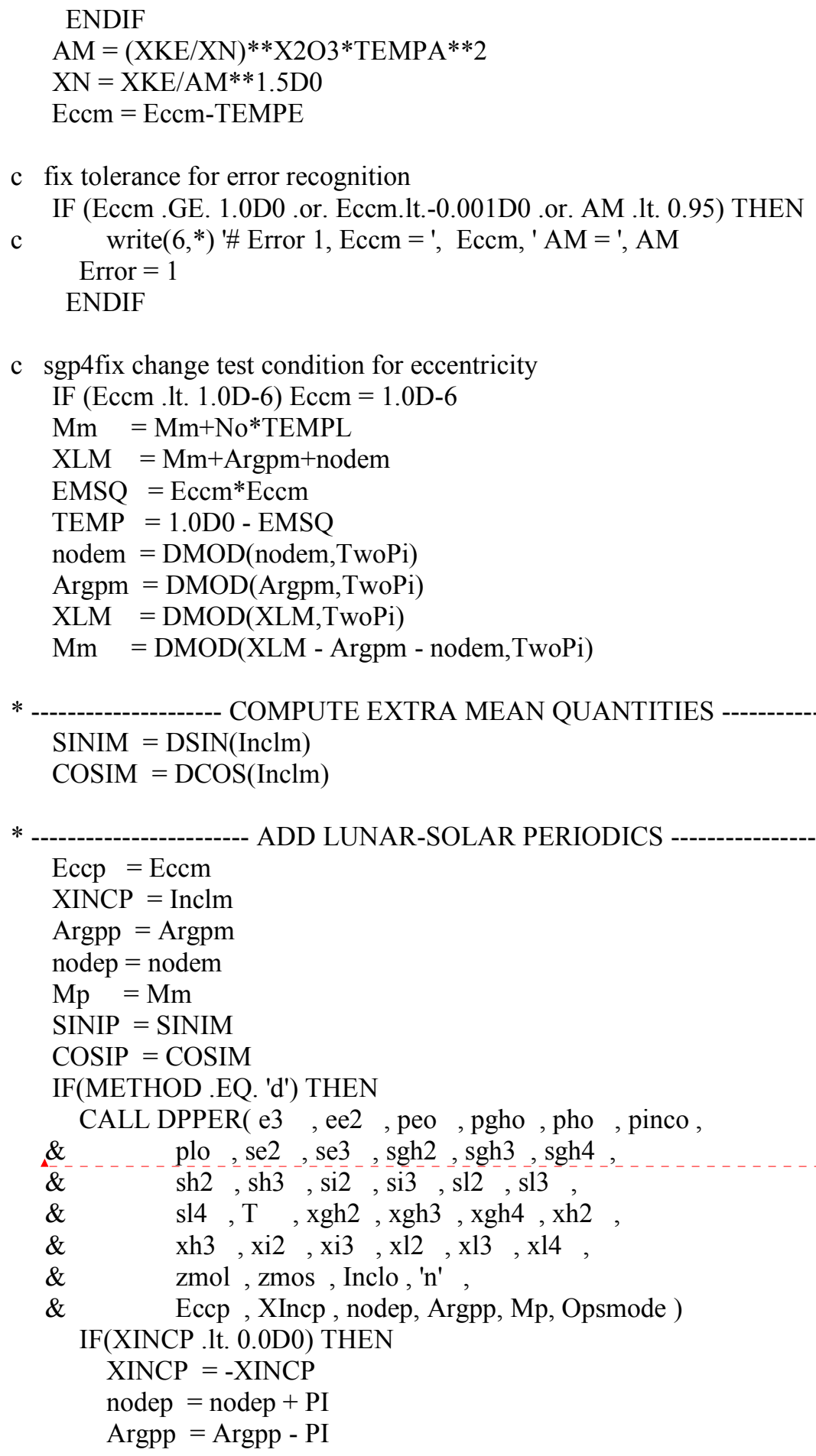




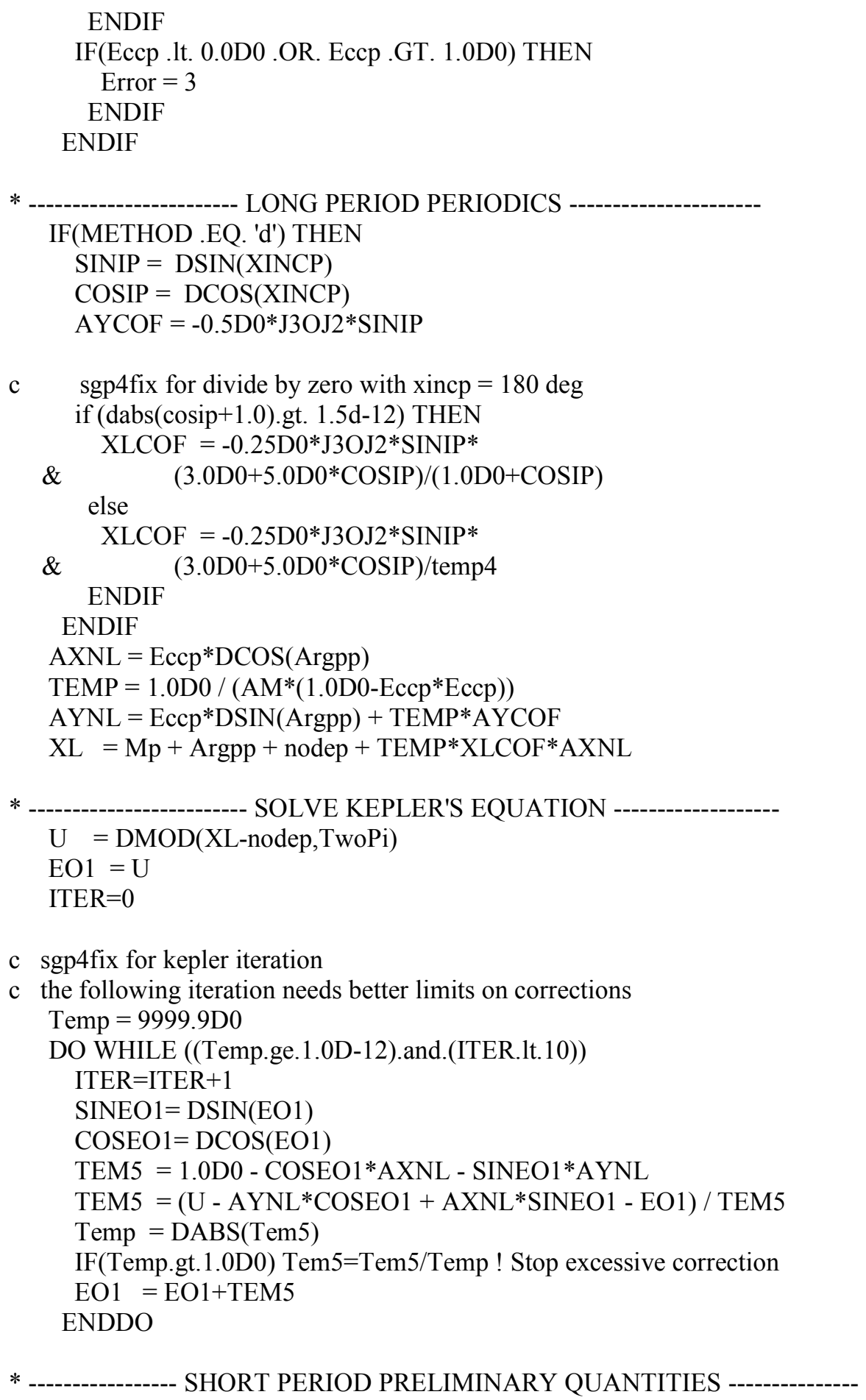


$\mathrm{ECOSE}=\mathrm{AXNL} * \mathrm{COSEO} 1+\mathrm{AYNL} * \mathrm{SINEO} 1$

$\mathrm{ESINE}=\mathrm{AXNL} * \mathrm{SINEO} 1-\mathrm{AYNL} * \mathrm{COSEO} 1$

$\mathrm{EL} 2=\mathrm{AXNL} * \mathrm{AXNL}+\mathrm{AYNL} * \mathrm{AYNL}$

$\mathrm{PL}=\mathrm{AM} *(1.0 \mathrm{D} 0-\mathrm{EL} 2)$

c semi-latus rectum $<0.0$

IF ( PL .1t. 0.0D0 ) THEN

Error $=4$

ELSE

$\mathrm{RL}=\mathrm{AM} *(1.0 \mathrm{D} 0-\mathrm{ECOSE})$

$\mathrm{RDOTL}=\mathrm{DSQRT}(\mathrm{AM}) * \mathrm{ESINE} / \mathrm{RL}$

$\mathrm{RVDOTL}=\mathrm{DSQRT}(\mathrm{PL}) / \mathrm{RL}$

$\mathrm{BETAL}=\mathrm{DSQRT}(1.0 \mathrm{D} 0-\mathrm{EL} 2)$

TEMP $=$ ESINE $/(1.0 \mathrm{D} 0+\mathrm{BETAL})$

SINU $=$ AM $/$ RL $*$ (SINEO1-AYNL-AXNL*TEMP $)$

$\mathrm{COSU}=\mathrm{AM} / \mathrm{RL} *(\mathrm{COSEO} 1-\mathrm{AXNL}+\mathrm{AYNL} * \mathrm{TEMP})$

$$
\mid
$$

$\mathrm{SU}=$ DATAN2 $($ SINU,COSU)

SIN $\overline{2} \bar{U}=\overline{(} \bar{C} \bar{S} \bar{U}+\overline{C O} \bar{S} \bar{U})^{*} \bar{S} \bar{N} \bar{U}$

$\mathrm{COS} 2 \mathrm{U}=1.0 \mathrm{D} 0-2.0 \mathrm{D} 0 * \mathrm{SINU} * \mathrm{SINU}$

$\mathrm{TEMP}=1.0 \mathrm{D} 0 / \mathrm{PL}$

TEMP $1=0.5 \mathrm{D} 0 * \mathrm{~J} 2 *$ TEMP

TEMP $2=$ TEMP $1 *$ TEMP

Formatted: French

Formatted: French

(France) UPDATE FOR SHORT PERIOD PERIODICS

IF(METHOD .EQ. 'd') THEN

COSISQ $=$ COSIP $*$ COSIP

CON41 $=3.0 \mathrm{D} 0 *$ COSISQ $-1.0 \mathrm{D} 0$

$\mathrm{X} 1 \mathrm{MTH} 2=1.0 \mathrm{D} 0-\mathrm{COSISQ}$

$\mathrm{X} 7 \mathrm{THM} 1=7.0 \mathrm{D} 0 * \mathrm{COSISQ}-1.0 \mathrm{D} 0$

ENDIF

$\mathrm{mr}=\mathrm{RL} *(1.0 \mathrm{D} 0-1.5 \mathrm{D} 0 * \mathrm{TEMP} 2 * \mathrm{BETAL} * \mathrm{CON} 41)+$

\& $\quad 0.5 \mathrm{D} 0 * \mathrm{TEMP} 1 * \mathrm{X} 1 \mathrm{MTH} 2 * \mathrm{COS} 2 \mathrm{U}$

SU $=$ SU $-0.25 \mathrm{D} 0 *$ TEMP $2 * X 7 T H M 1 *$ SIN $2 \mathrm{U}$

$\mathrm{XNODE}=$ nodep $+1.5 \mathrm{D} 0 * \mathrm{TEMP} 2 * \mathrm{COSIP}^{*} \mathrm{~S} I \mathrm{~N} 2 \mathrm{U}$

$\mathrm{XINC}=\mathrm{XINCP}+1.5 \mathrm{D} 0 *$ TEMP $2 *$ COSIP $*$ SINIP*COS2U

$\mathrm{mv}=\mathrm{RDOTL}-\mathrm{XN} * \mathrm{TEMP} 1 * \mathrm{X} 1 \mathrm{MTH} 2 * \mathrm{SIN} 2 \mathrm{U} / \mathrm{XKE}$

$\mathrm{RVDOT}=\mathrm{RVDOTL}+\mathrm{XN} * \mathrm{TEMP} 1 *(\mathrm{X} 1 \mathrm{MTH} 2 * \mathrm{COS} 2 \mathrm{U}+1.5 \mathrm{D} 0 * \mathrm{CON} 41) / \mathrm{XKE}$

Formatted: French

(France)

SINSU $=\operatorname{DSIN}(\mathrm{SU})$

ORIENTATION VECTORS

$\mathrm{COSSU}=\mathrm{DCOS}(\mathrm{SU})$

$\mathrm{SNOD}=\mathrm{DSIN}(\mathrm{XNODE})$

$\mathrm{CNOD}=\mathrm{DCOS}(\mathrm{XNODE})$

$\mathrm{SINI}=\operatorname{DSIN}(\mathrm{XINC})$

$\mathrm{COSI}=\operatorname{DCOS}(\mathrm{XINC})$

$\mathrm{XMX}=-\mathrm{SNOD}^{*} \mathrm{COSI}$

Formatted: French

(France) 


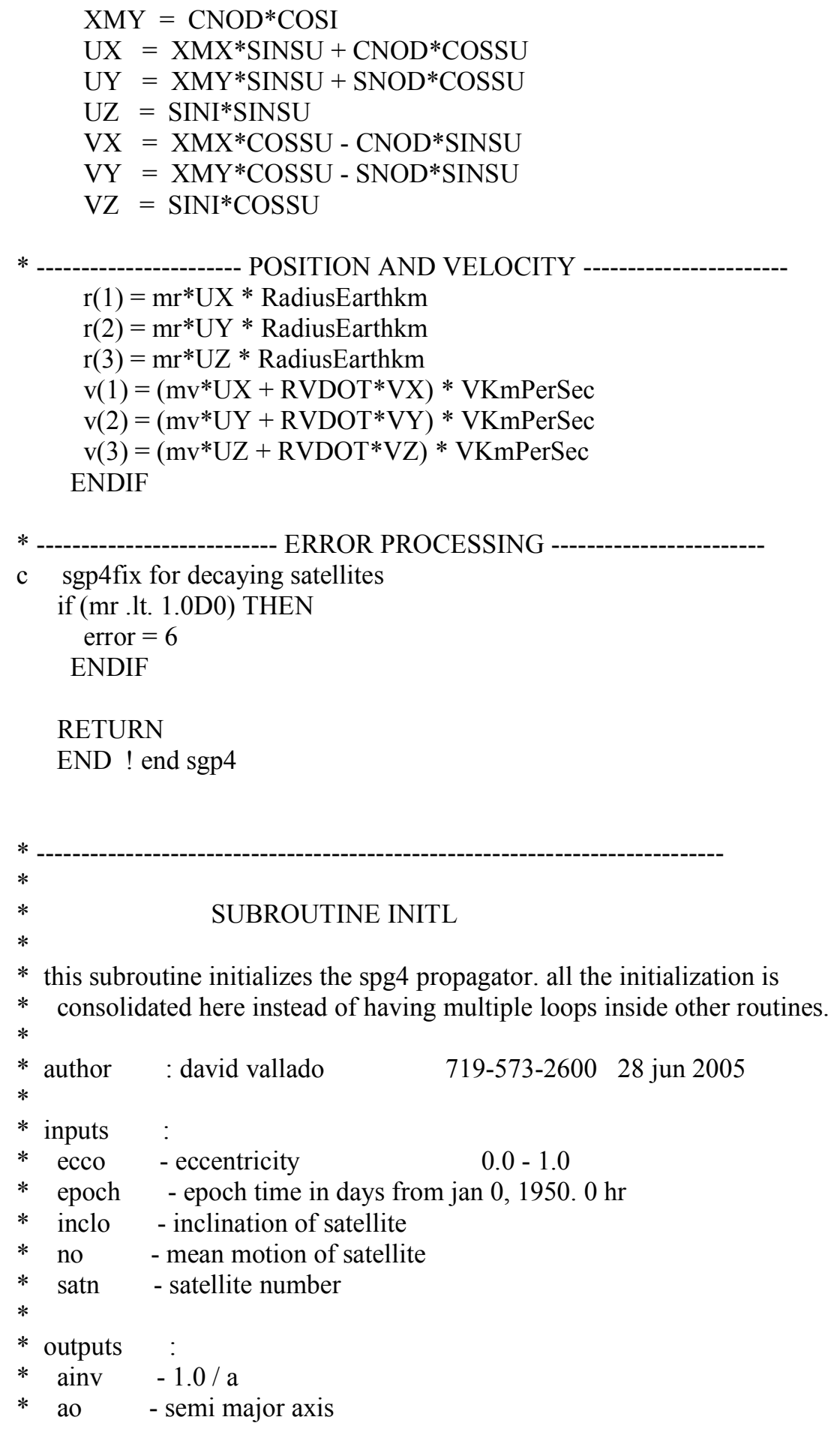




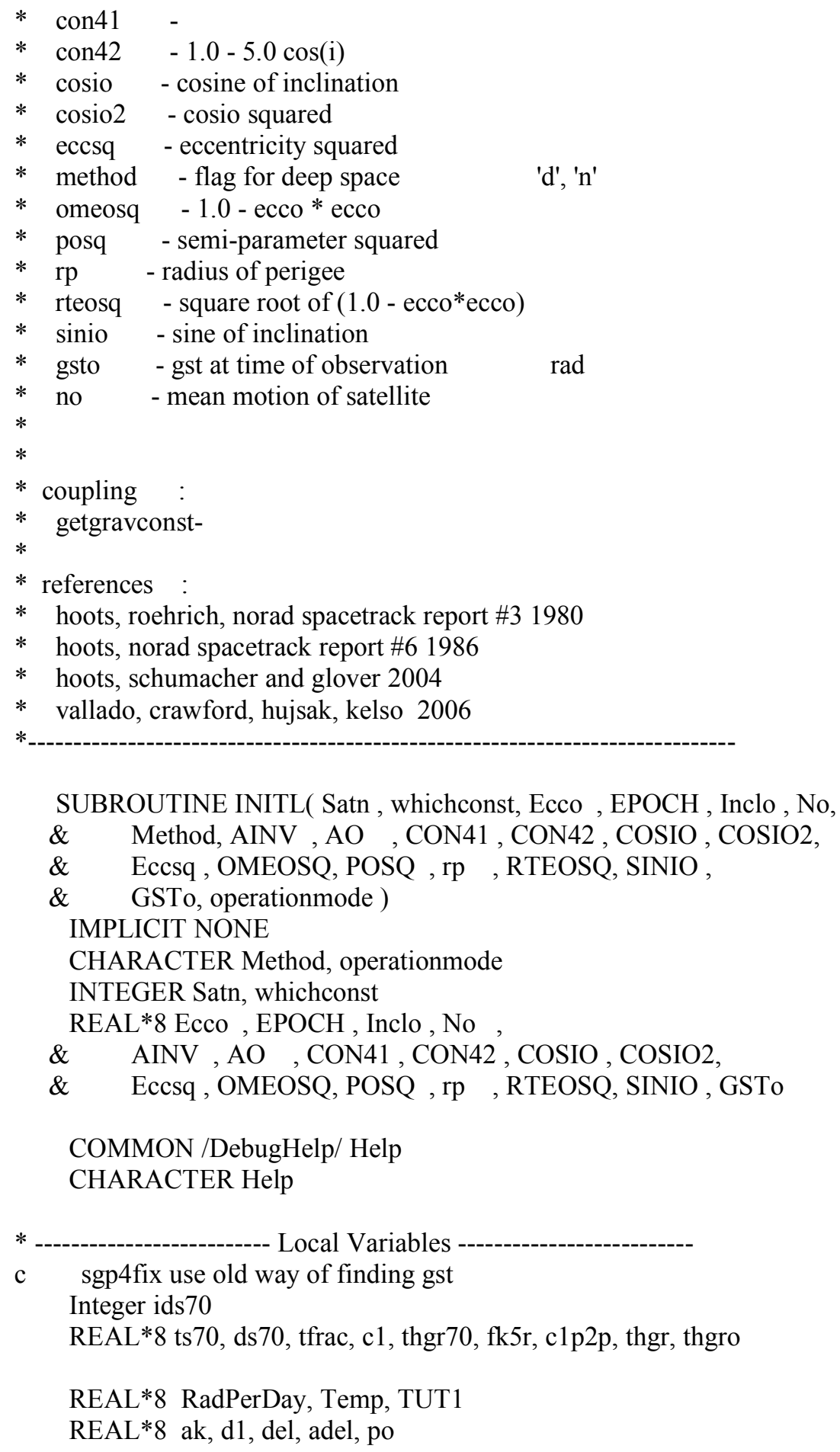

SUBROUTINE INITL( Satn, whichconst, Ecco , EPOCH, Inclo, No,

\& Method, AINV , AO , CON41, CON42, COSIO, COSIO2,

\& Eccsq, OMEOSQ, POSQ , rp , RTEOSQ, SINIO,

\& GSTo, operationmode )

IMPLICIT NONE

CHARACTER Method, operationmode

INTEGER Satn, whichconst

REAL*8 Ecco , EPOCH , Inclo, No ,

$\& \quad$ AINV $, \mathrm{AO}, \mathrm{CON} 41, \mathrm{CON} 42, \mathrm{COSIO}, \mathrm{COSIO} 2$,

\& Eccsq, OMEOSQ, POSQ, rp , RTEOSQ, SINIO, GSTo

COMMON /DebugHelp/ Help

CHARACTER Help

* --

c sgp4fix use old way of finding gst

Integer ids70

REAL*8 ts70, ds 70, tfrac, c1, thgr70, fk5r, c1p2p, thgr, thgro

REAL*8 RadPerDay, Temp, TUT1

REAL*8 ak, d1, del, adel, po 
REAL*8 X2o3, J2, XKE, tumin, mu, radiusearthkm, j3, j4, j3oj2

INCLUDE 'ASTMATH.CMN'

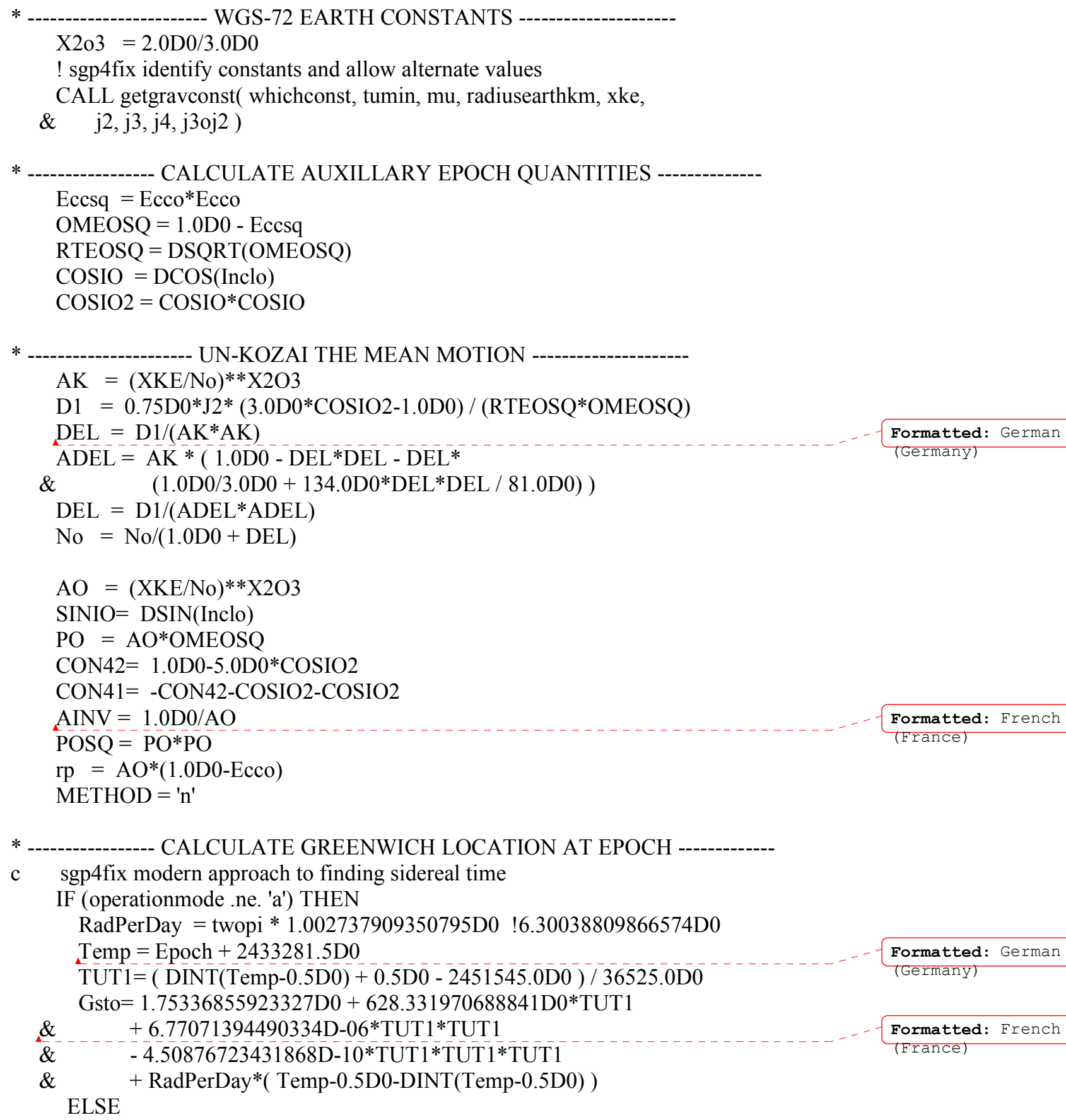


! sgp4fix use old way of finding gst

! count integer number of days from 0 jan 1970

TS70 $=$ EPOCH-7305.0D0

IDS70 $=$ TS70 $+1.0 \mathrm{D}-8$

TFRAC $=$ TS70-IDS70

! find greenwich location at epoch

$\mathrm{C} 1=1.72027916940703639 \mathrm{D}-2$

THGR70 $=1.7321343856509374 \mathrm{D} 0$

$\mathrm{FK} 5 \mathrm{R}=5.07551419432269442 \mathrm{D}-15$

$\mathrm{C} 1 \mathrm{P} 2 \mathrm{P}=\mathrm{C} 1+\mathrm{TWOPI}$

gsto $=$ THGR70+C $1 *$ IDS70+C1P2P*TFRAC + TS70*TS70*FK5R

ENDIF

! ------------ Check quadrants

Gsto $=$ DMOD $($ Gsto, TwoPi $)$

IF ( Gsto .lt. 0.0D0 ) THEN

Gsto $=$ Gsto + TwoPi

ENDIF

RETURN

END ! end initl

$*$

$*$

* $\quad$ SUBROUTINE DPPER

$*$

* This Subroutine provides deep space long period periodic contributions

* to the mean elements. by design, these periodics are zero at epoch.

* this used to be dscom which included initialization, but it's really a

* recurring function.

* author : david vallado 719-573-2600 28 jun 2005

$*$

* inputs :

* e3 -

* ee2 -

* peo -

* pgho -

* pho

* pinco

plo -

* se2, se3, Sgh2, Sgh3, Sgh4, Sh2, Sh3, Si2, Si3, S12, S13, S14 -

$* \mathrm{t}$

* xh2, xh3, xi2, xi3, x12, x13, x14 -

* $\mathrm{zmol}$

* zmos -

Formatted: French

(France)

Formatted: German

(Germany) 
REAL*8 Zel , Zes , Znl , Zns

!COMMON /DebugHelp/ Help

CHARACTER Help

INCLUDE 'ASTMATH.CMN'

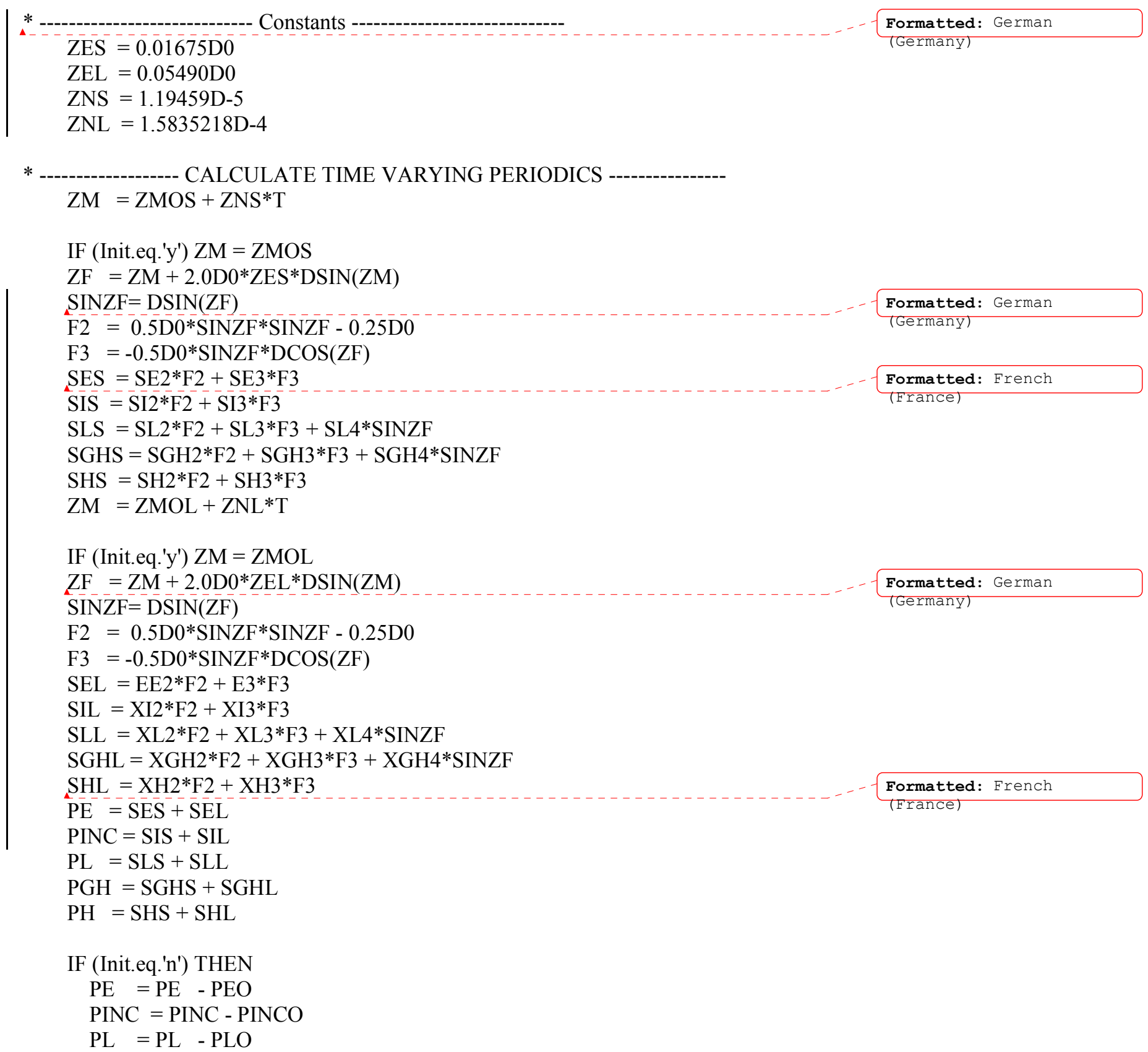




$$
\begin{aligned}
& \text { PGH = PGH }- \text { PGHO } \\
& \text { PH = PH }- \text { PHO } \\
& \text { Inclp = Inclp + PINC } \\
& \text { Eccp = Eccp + PE } \\
& \text { SINIP = DSIN(Inclp) } \\
& \text { COSIP = DCOS(Inclp) }
\end{aligned}
$$

c sgp4fix for lyddane choice

c strn3 used original inclination - this is technically feasible

c gsfc used perturbed inclination - also technically feasible

c probably best to readjust the 0.2 limit value and limit discontinuity

c $0.2 \mathrm{rad}=11.45916 \mathrm{deg}$

c use next line for original strn3 approach and original inclination

c IF (inclo.ge.0.2D0) THEN

c use next line for gsfc version and perturbed inclination

IF (Inclp.ge.0.2D0) THEN

$$
\begin{aligned}
& \mathrm{PH}=\mathrm{PH} / \mathrm{SINIP} \\
& \mathrm{PGH}=\mathrm{PGH}-\text { COSIP } * \text { PH } \\
& \text { Argpp }=\text { Argpp }+ \text { PGH } \\
& \text { nodep }=\text { nodep }+ \text { PH } \\
& \text { Mp }=\text { Mp }+ \text { PL } \\
& \text { ELSE }
\end{aligned}
$$




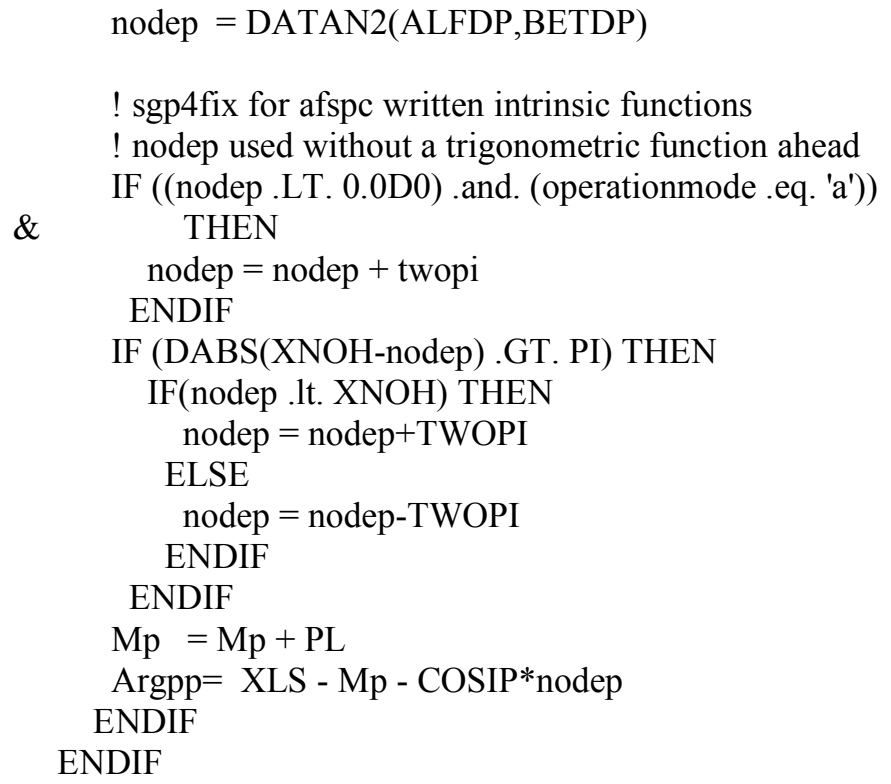




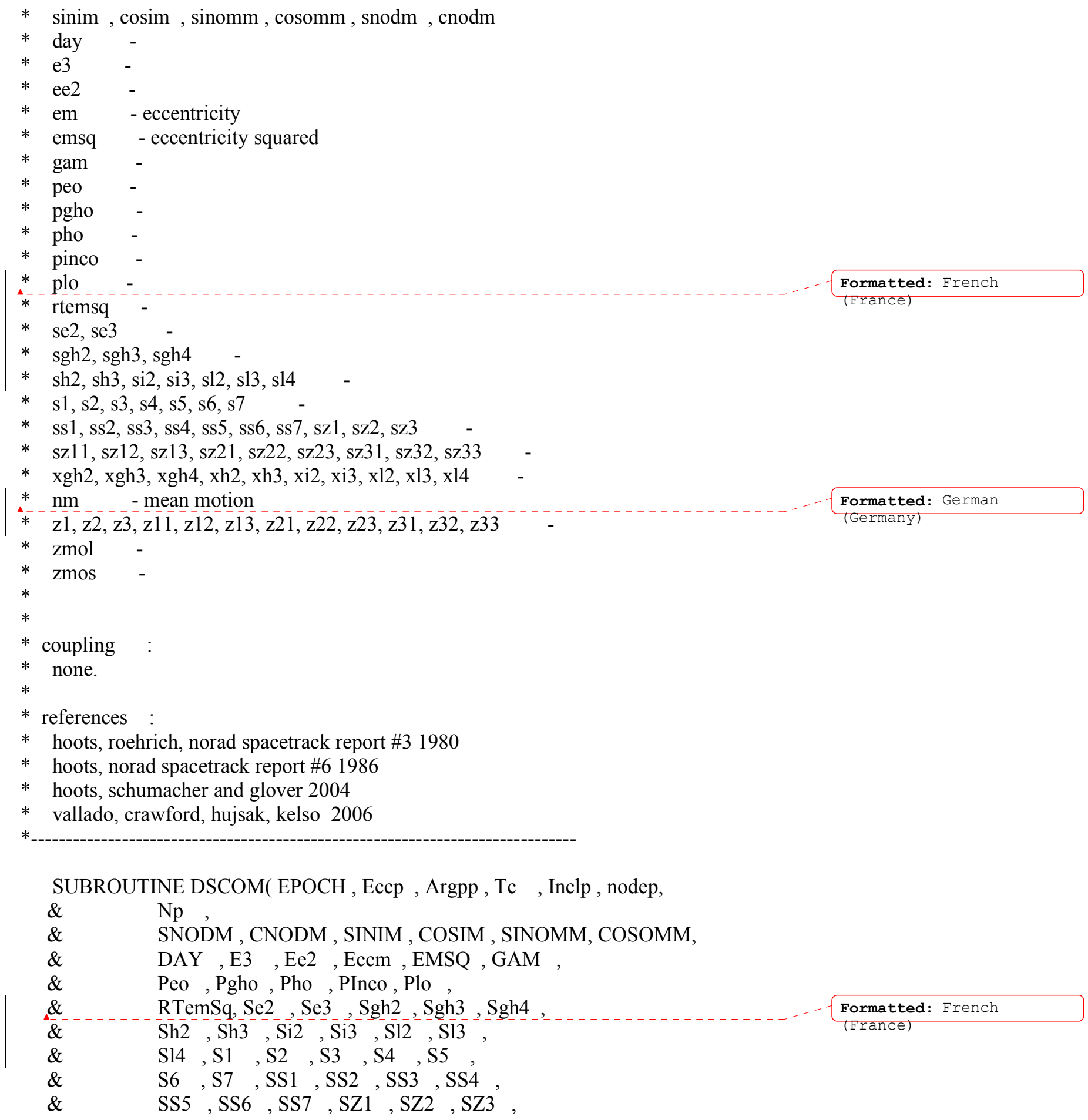




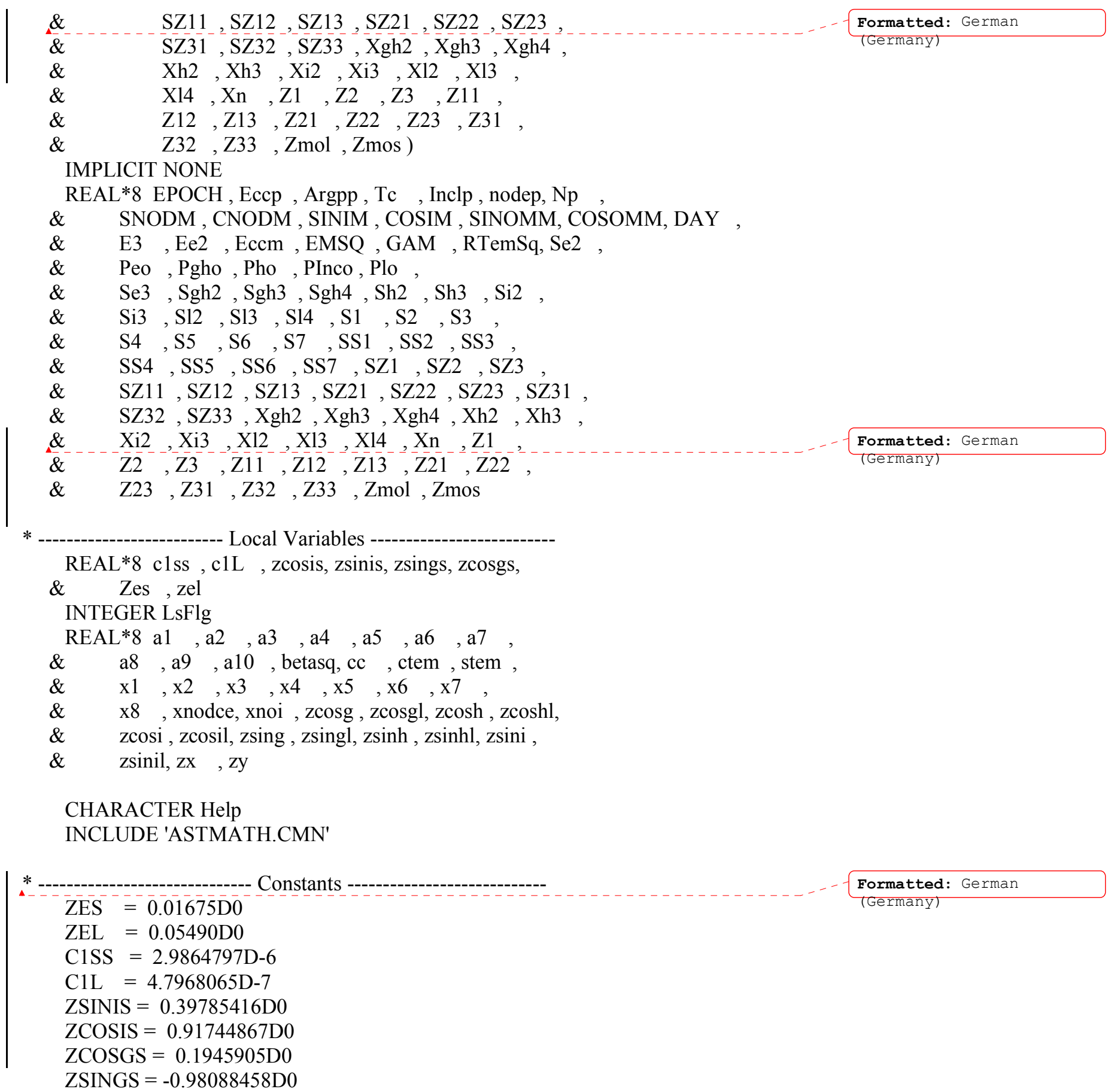




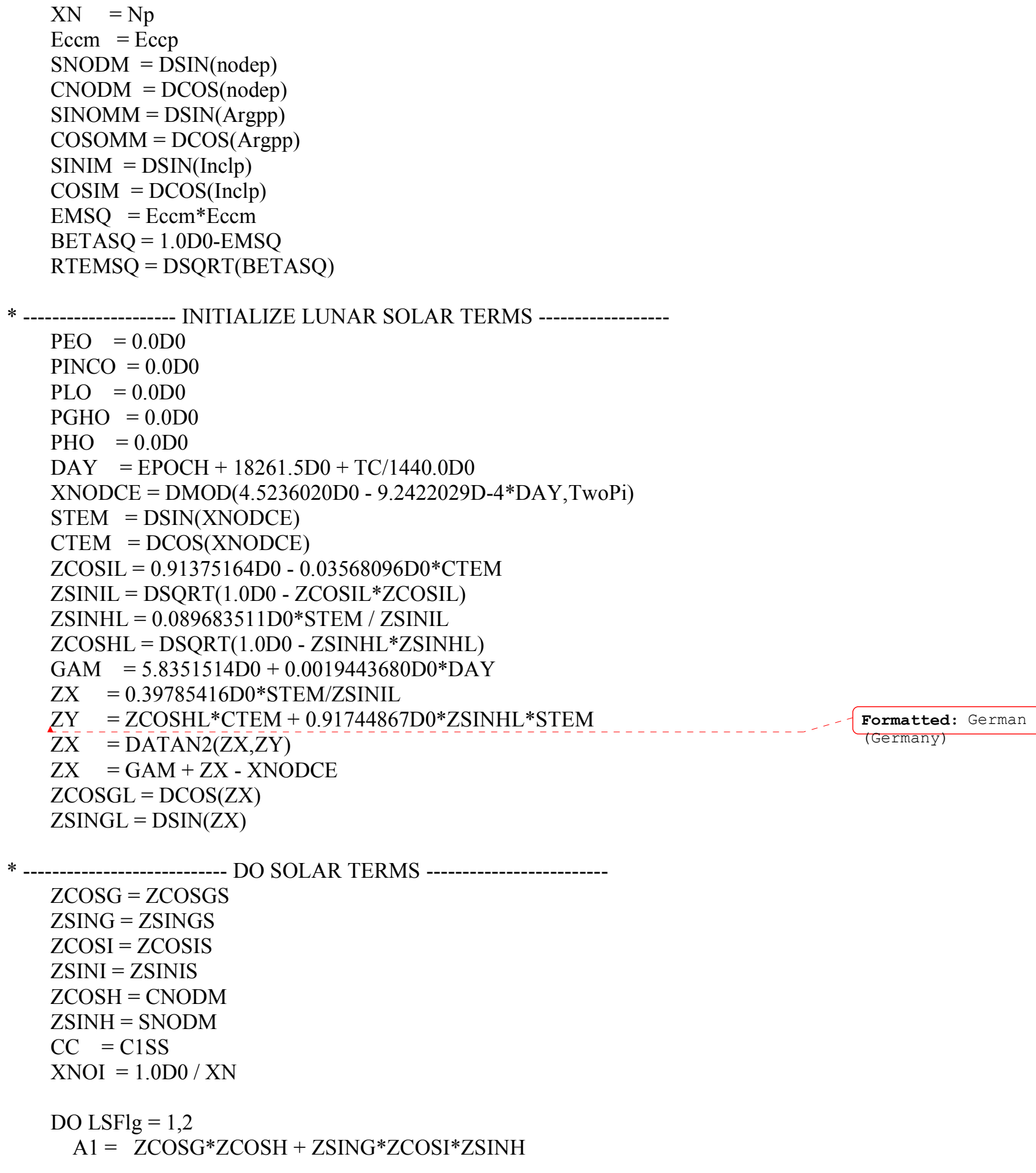

$$
\mathrm{A} 1=\mathrm{ZCOSG} * \mathrm{ZCOSH}+\mathrm{ZSING} * \mathrm{ZCOSI} * \mathrm{ZSINH}
$$




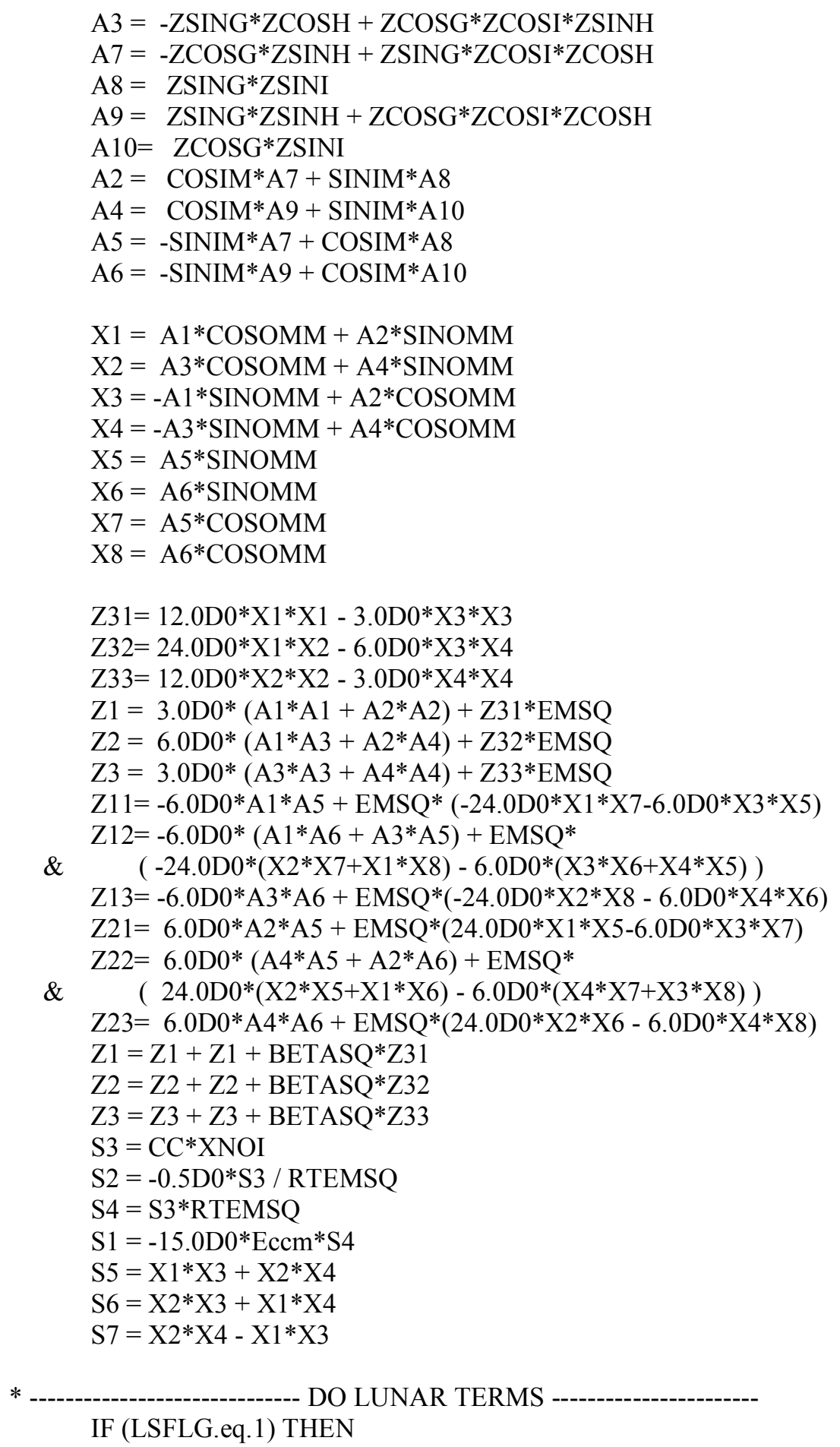




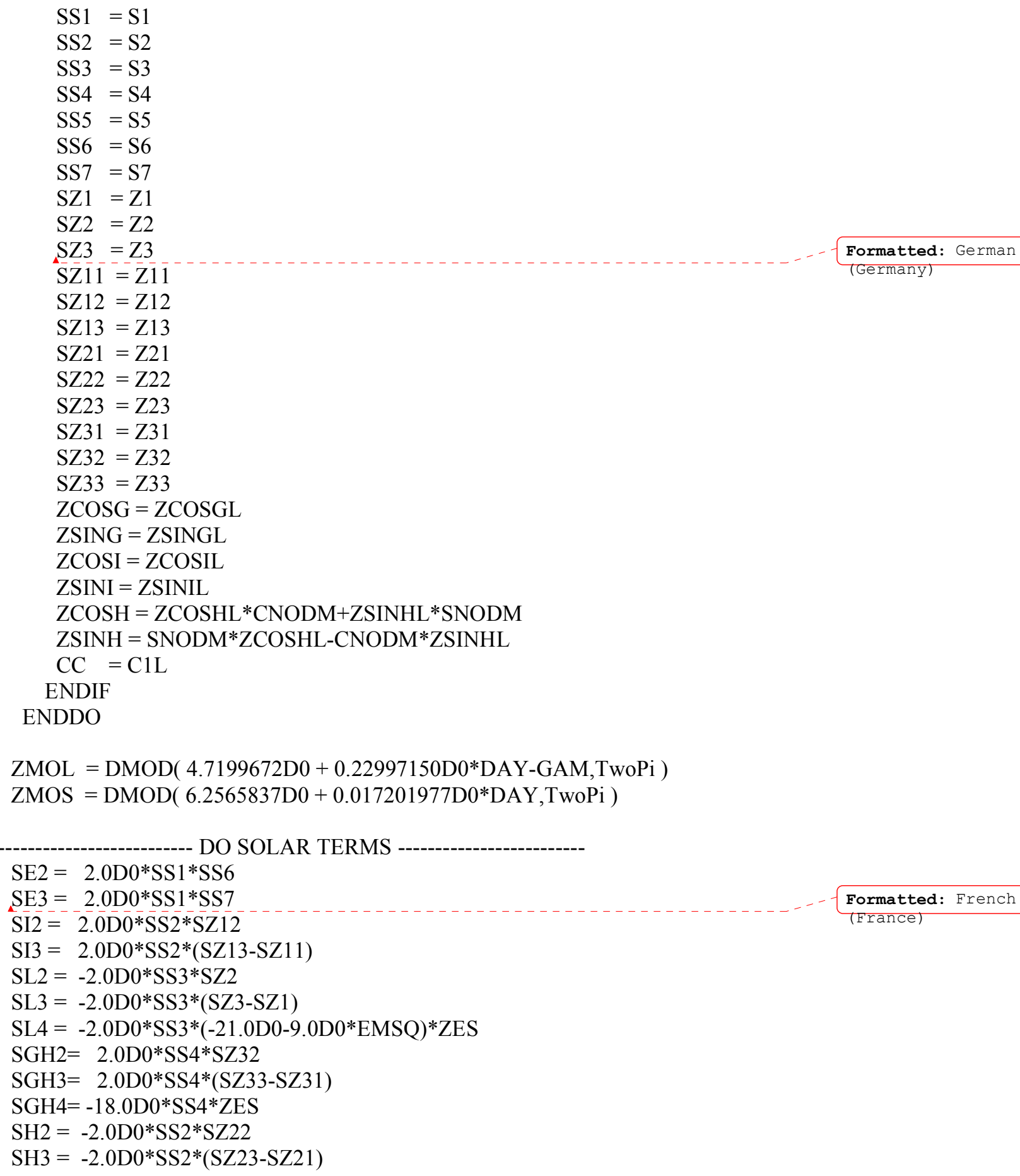




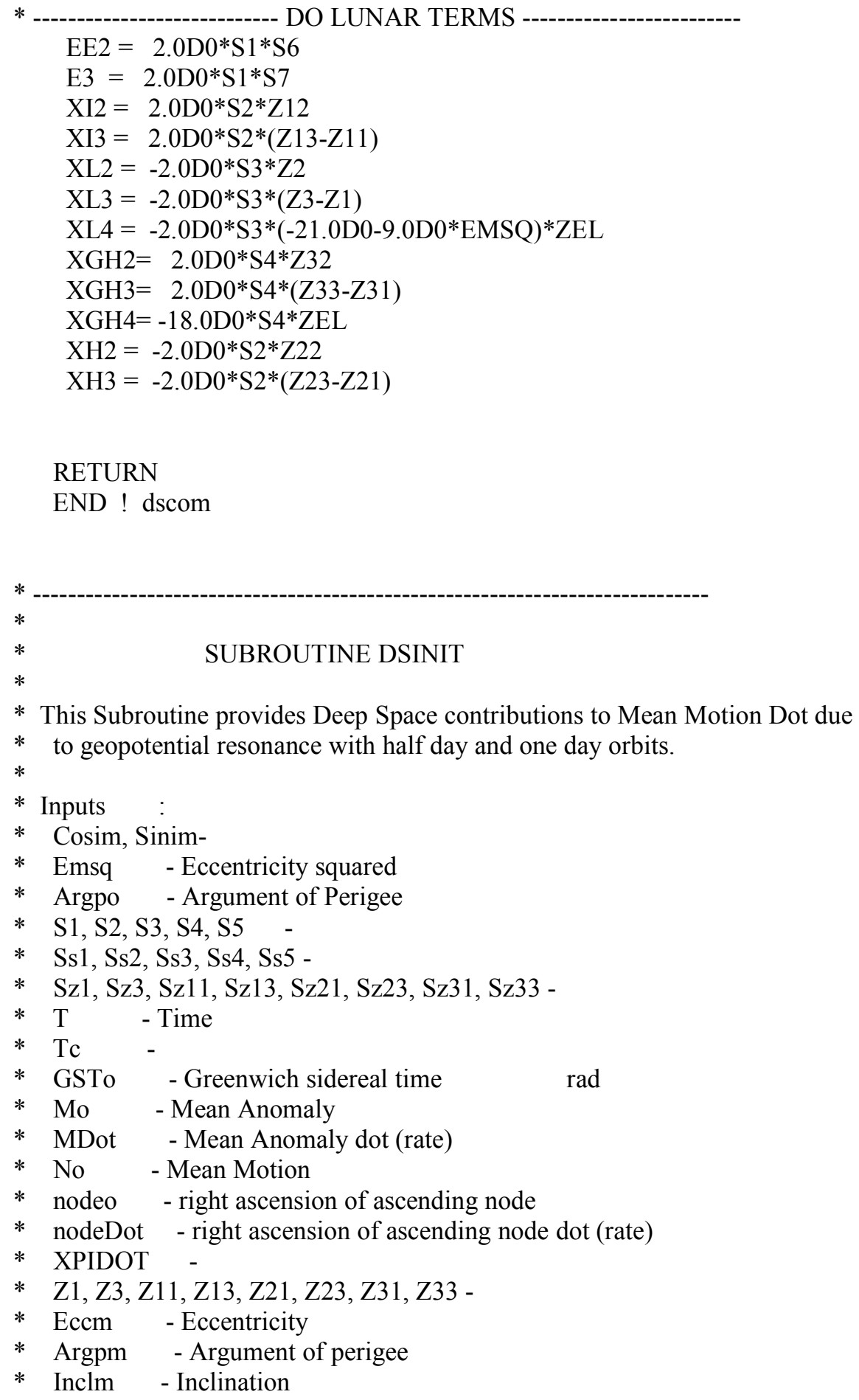

RETURN

END ! dscom 


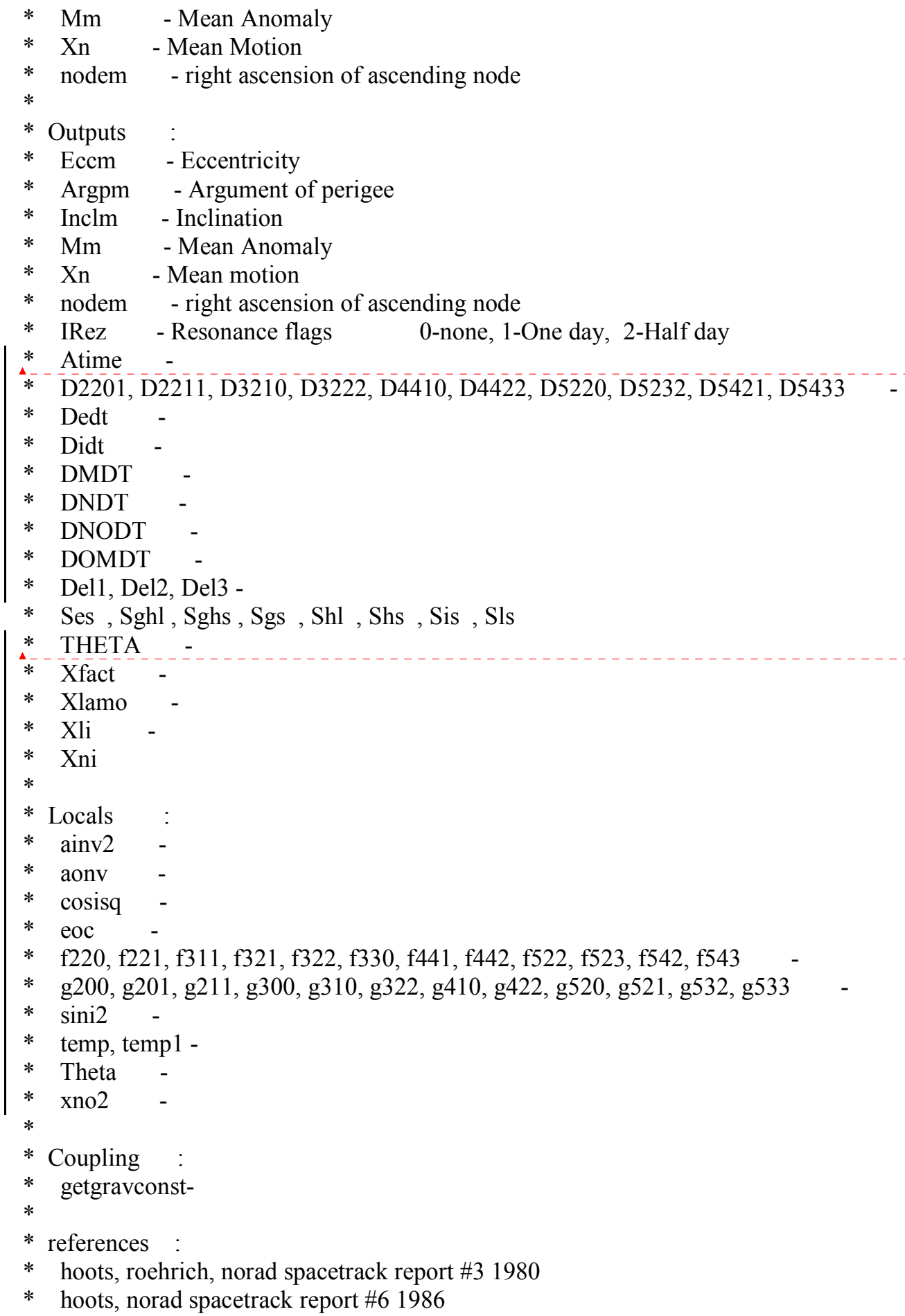


* hoots, schumacher and glover 2004

* vallado, crawford, hujsak, kelso 2006

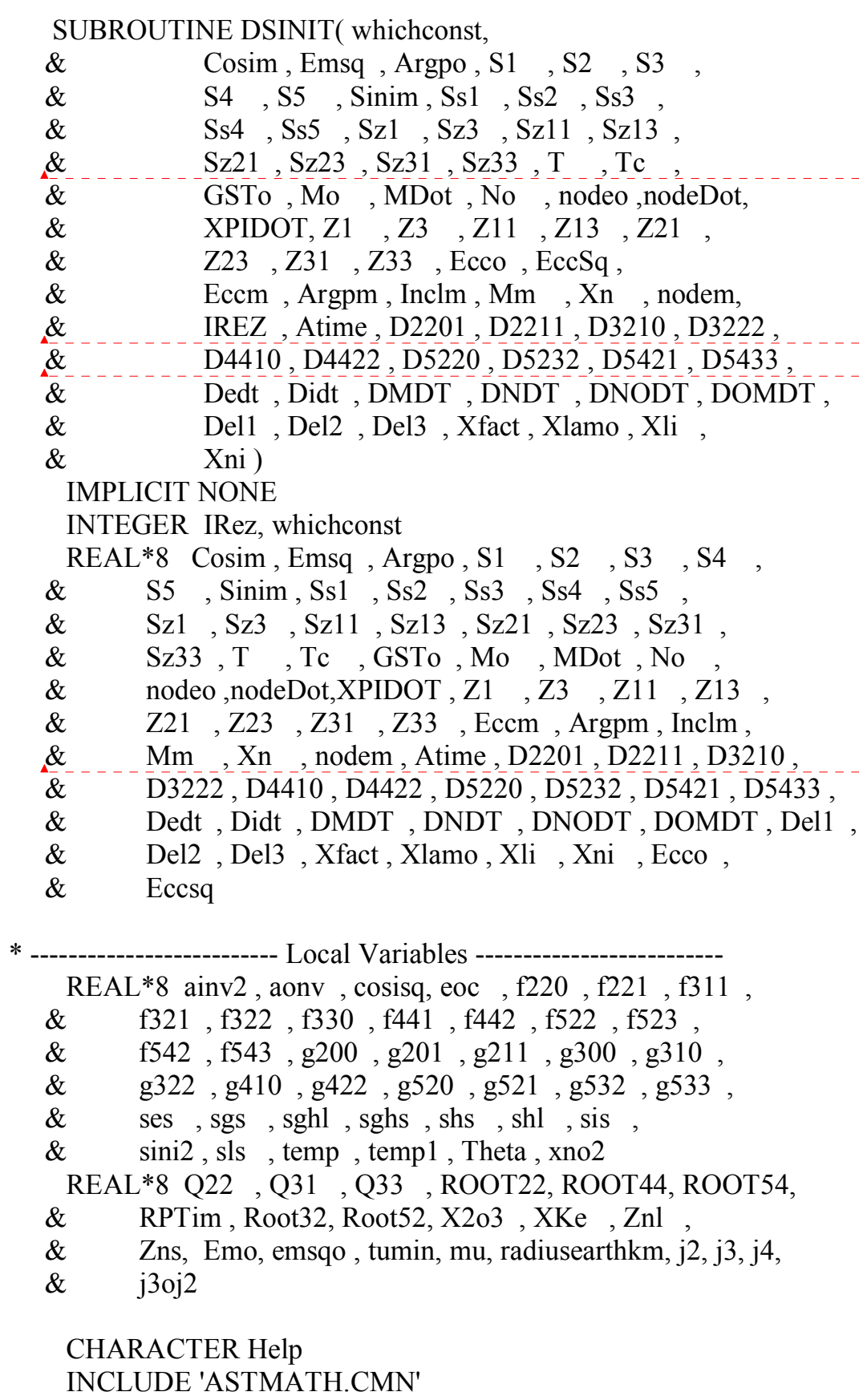




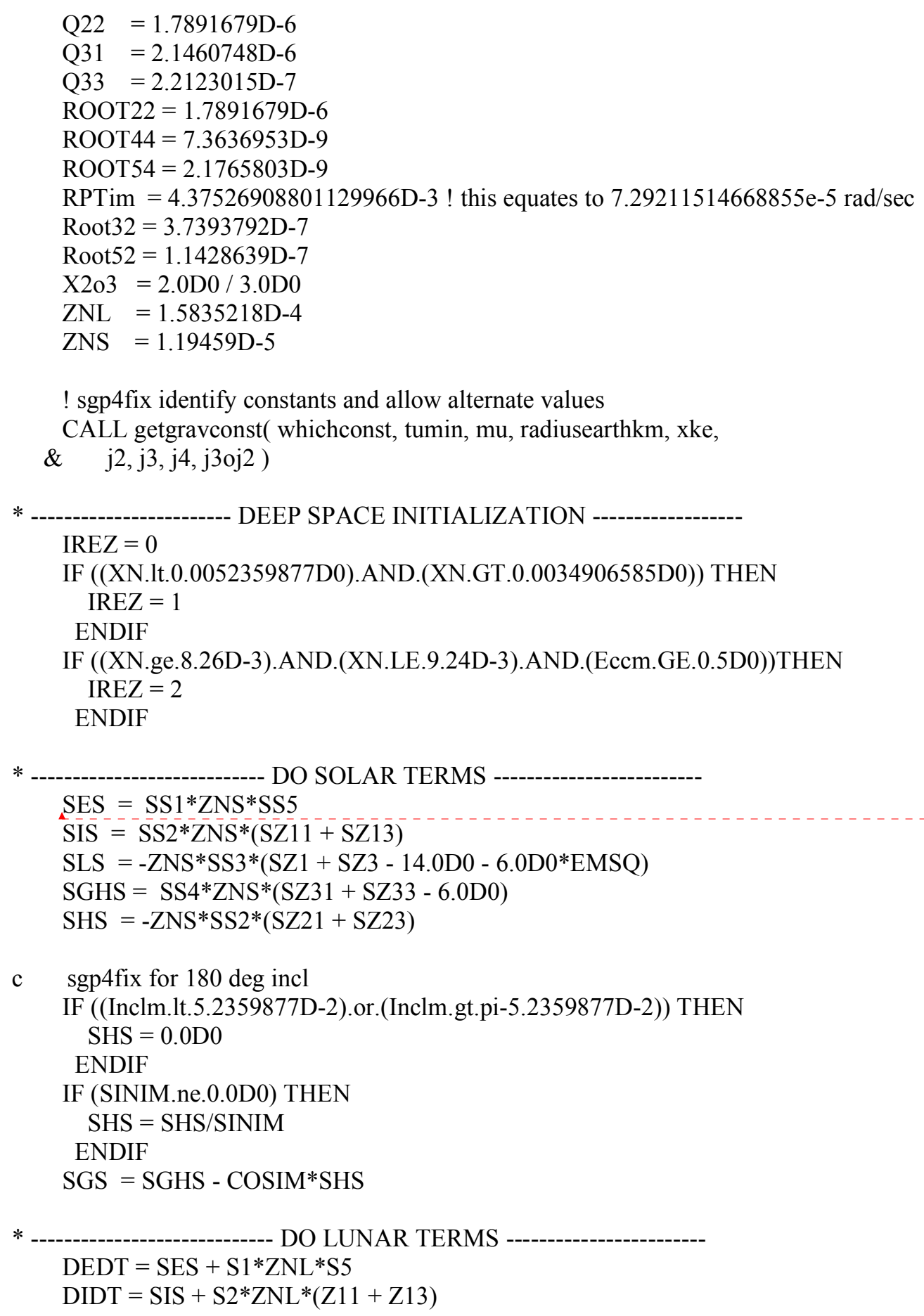


$\mathrm{DMDT}=\mathrm{SLS}-\mathrm{ZNL} * \mathrm{~S} 3 *(\mathrm{Z} 1+\mathrm{Z3}-14.0 \mathrm{D} 0-6.0 \mathrm{D} 0 * \mathrm{EMSQ})$

$\mathrm{SGHL}=\mathrm{S} 4 * \mathrm{ZNL} *(\mathrm{Z} 31+\mathrm{Z} 33-6.0 \mathrm{D} 0)$

$\mathrm{SHL}=-\mathrm{ZNL} * \mathrm{~S} 2 *(\mathrm{Z} 21+\mathrm{Z} 23)$

c sgp4fix for $180 \mathrm{deg}$ incl

IF ((Inclm.1t.5.2359877D-2).or.(Inclm.gt.pi-5.2359877D-2)) THEN

$\mathrm{SHL}=0.0 \mathrm{D} 0$

ENDIF

$\mathrm{DOMDT}=\mathrm{SGS}+\mathrm{SGHL}$

DNODT $=$ SHS

IF (SINIM .ne. 0.0D0) THEN

DOMDT $=$ DOMDT-COSIM/SINIM $*$ SHL

DNODT $=$ DNODT + SHL/SINIM

ENDIF

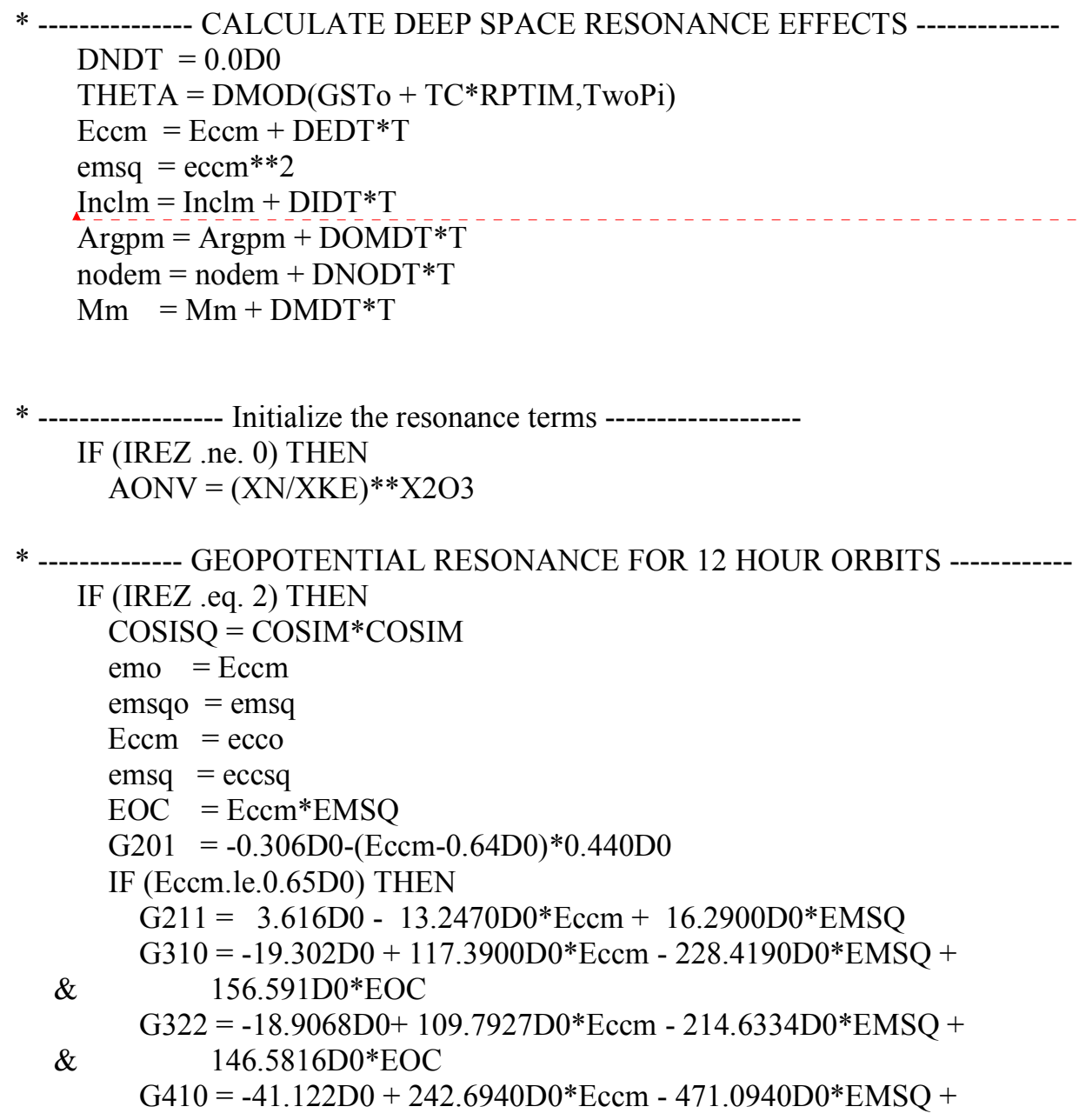




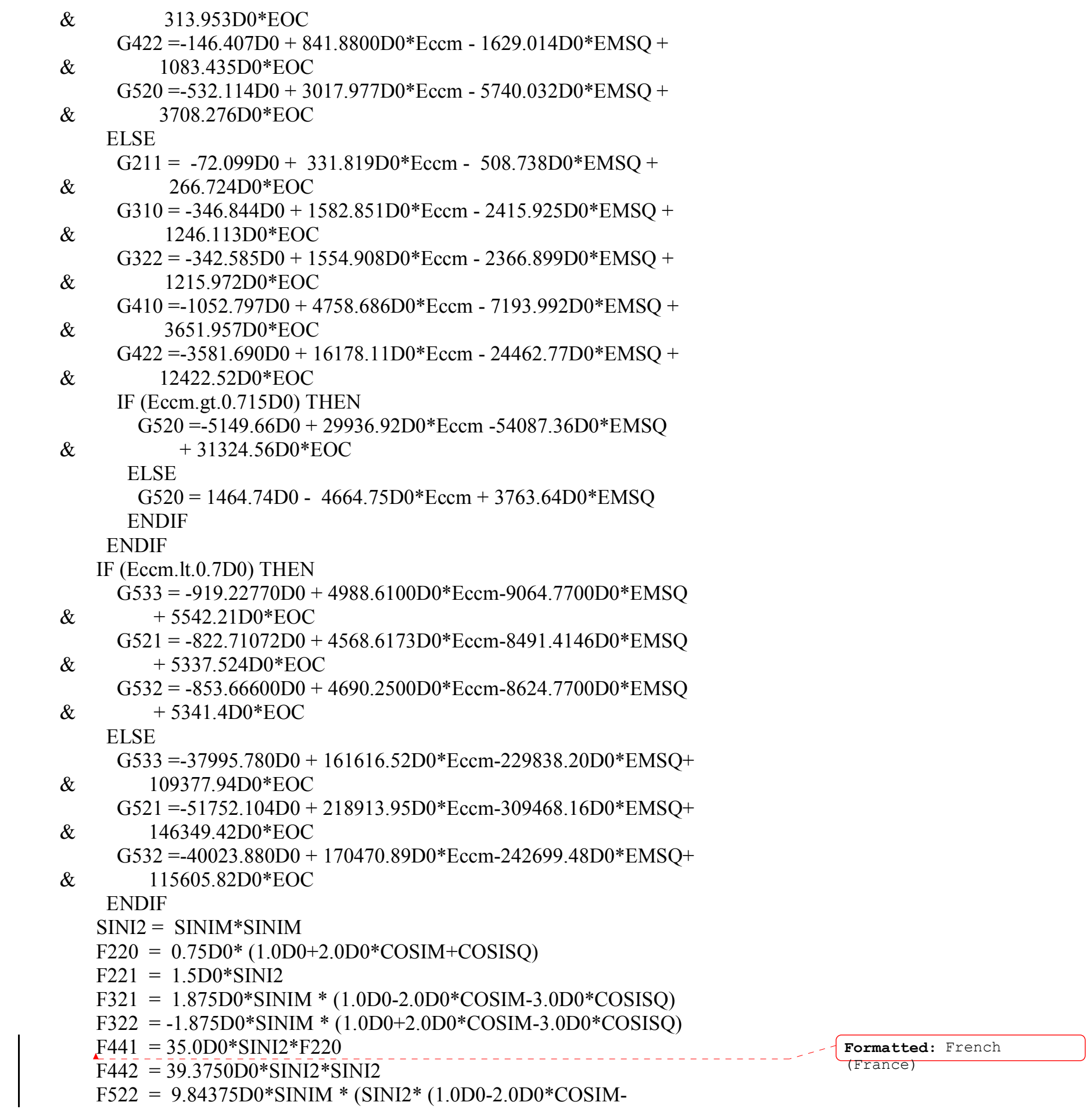




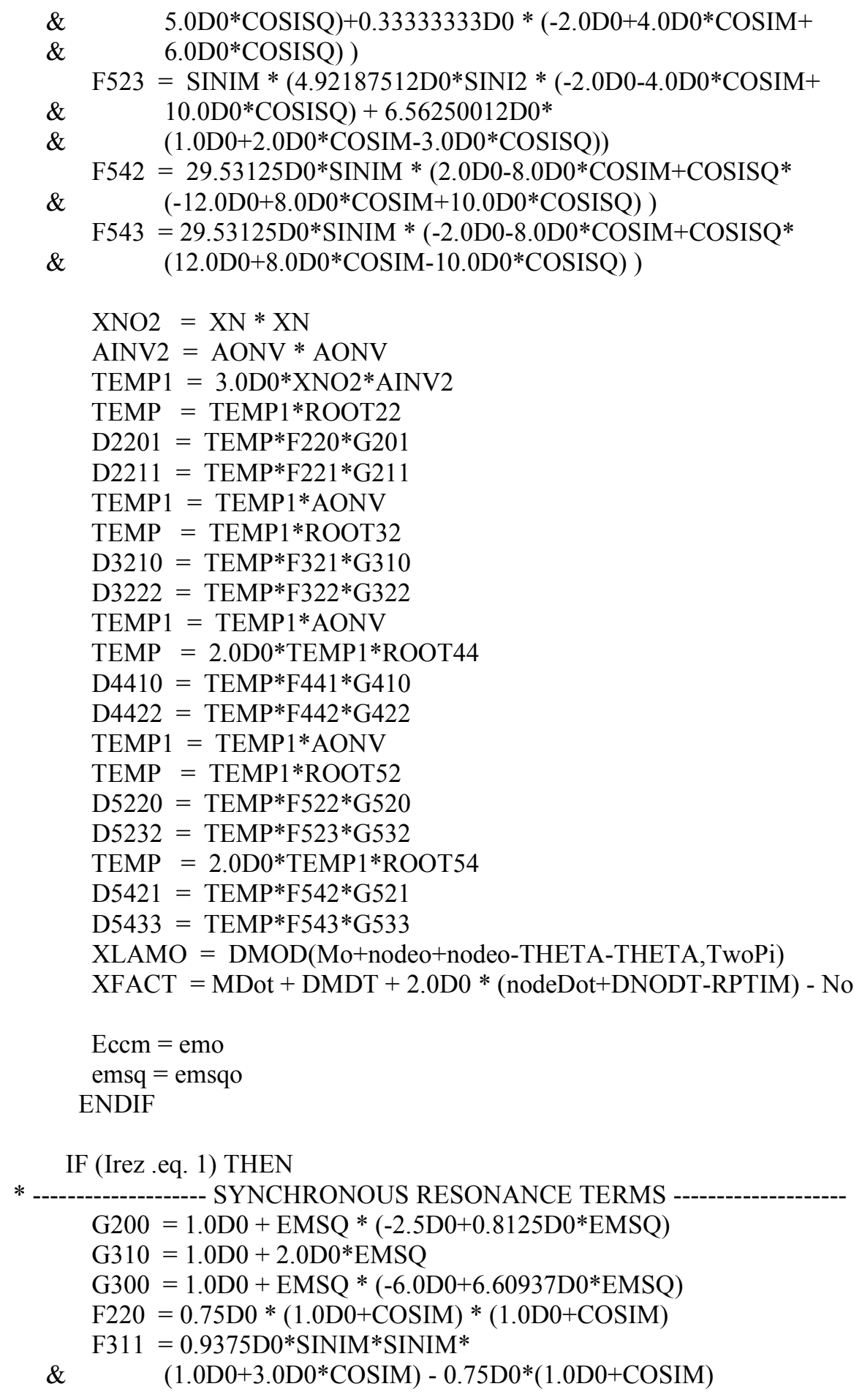




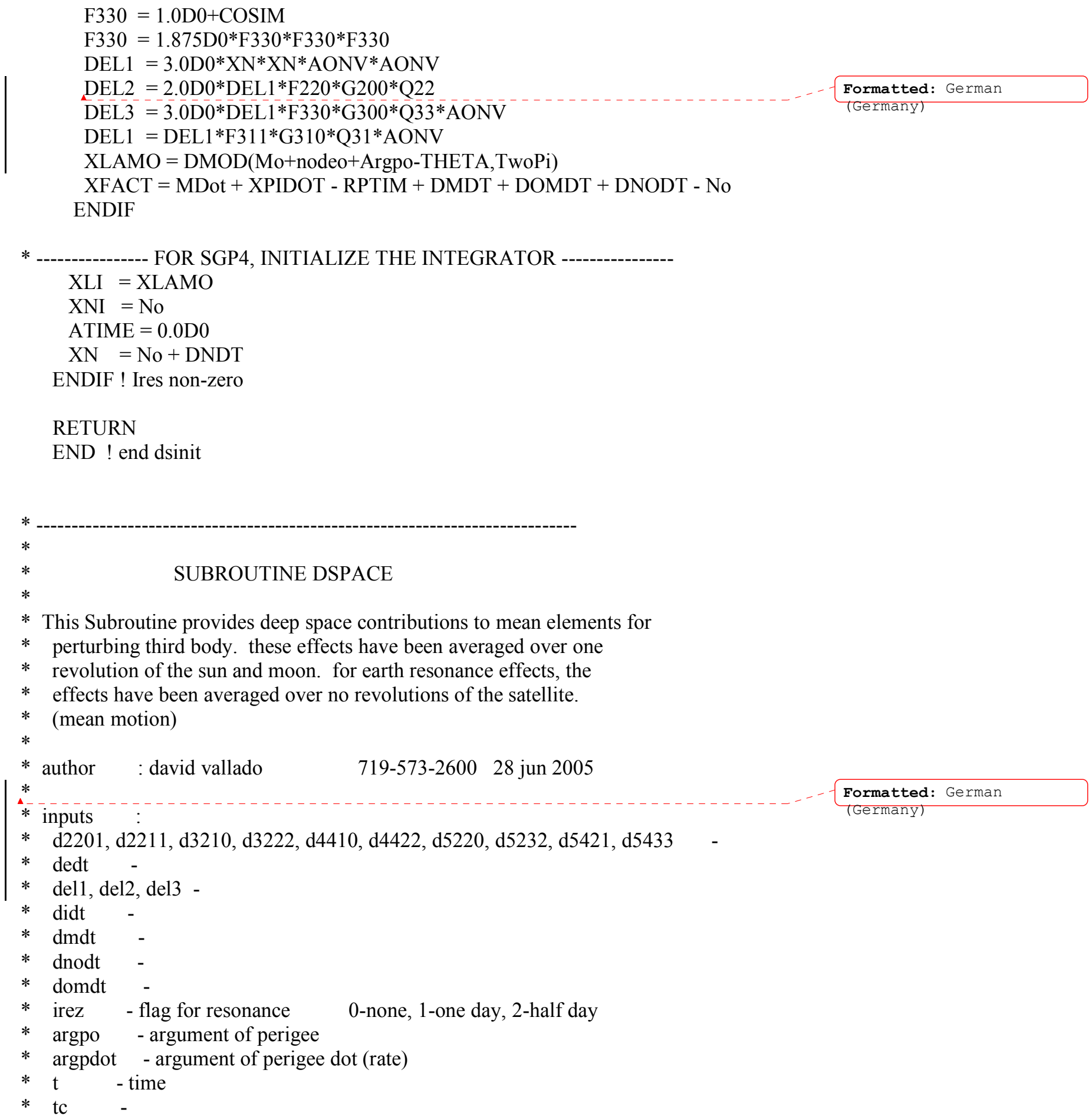




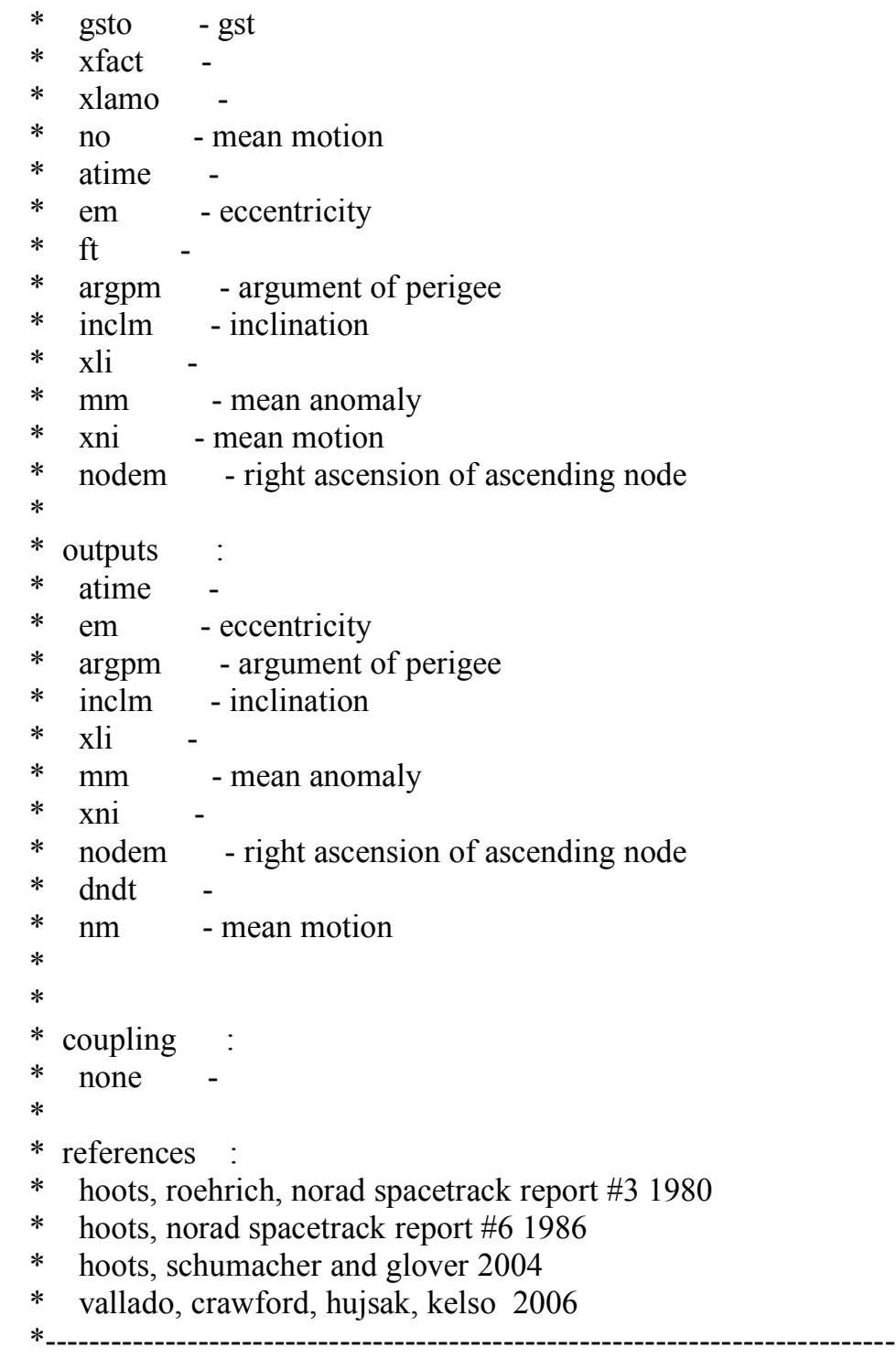

SUBROUTINE DSPACE( IRez , D2201 , D2211 , D3210 , D3222 , D4410 , \& $\quad$ D4422, D5220, D5232, D5421, D5433, Dedt ,

\& Del1 , Del2 , Del3 , Didt , Dmdt , Dnodt ,

\& Domdt, Argpo, ArgpDot, T , TC , GSTo ,

\& Xfact, Xlamo, No ,

\& Atime, Eccm, Argpm, Inclm, Xli , Mm ,

$\& \quad \mathrm{XNi}$, nodem, Dndt , XN )

IMPLICIT NONE

INTEGER IRez

Formatted: French (France)

Formatted: German

(Germany) 


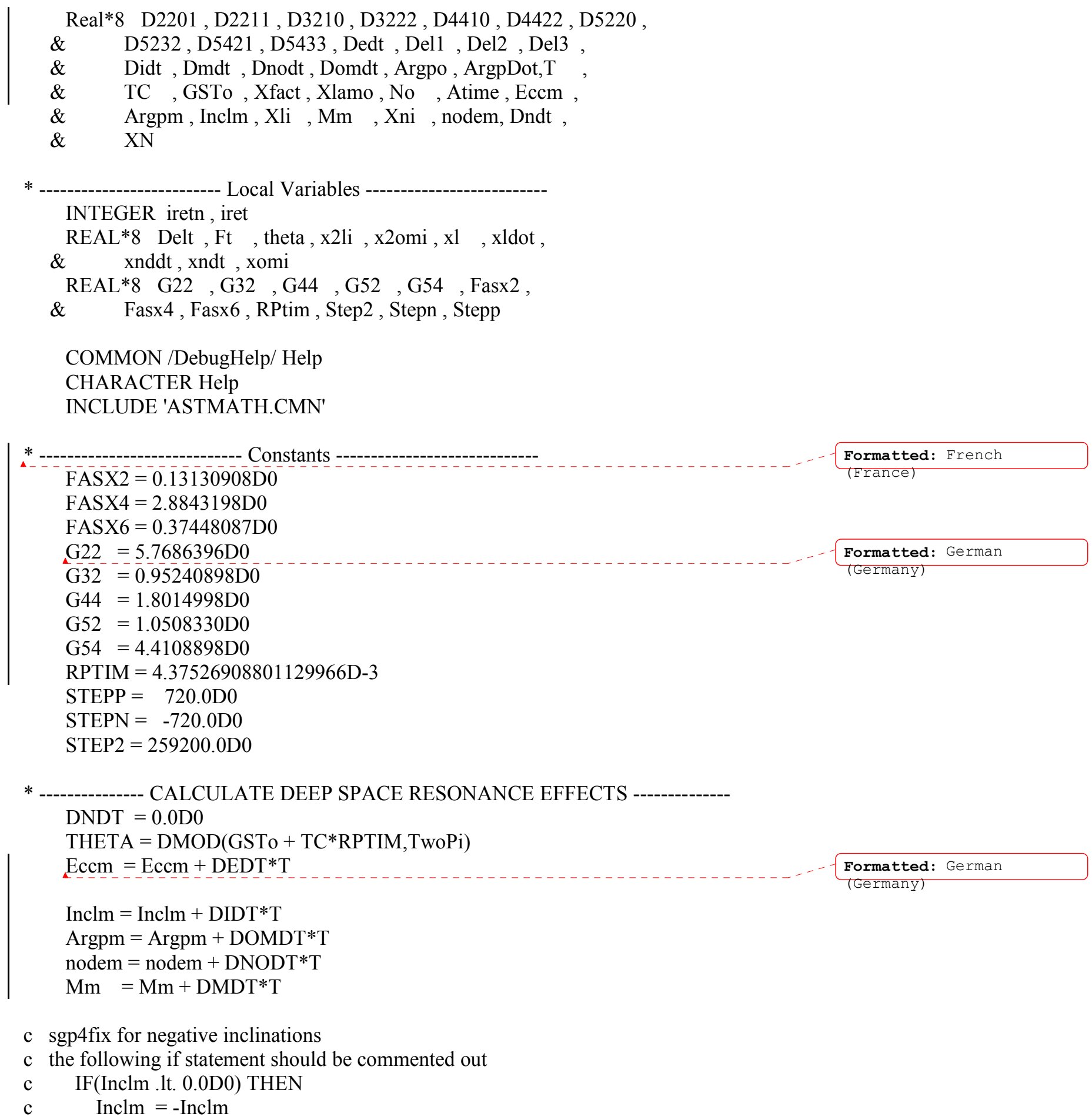




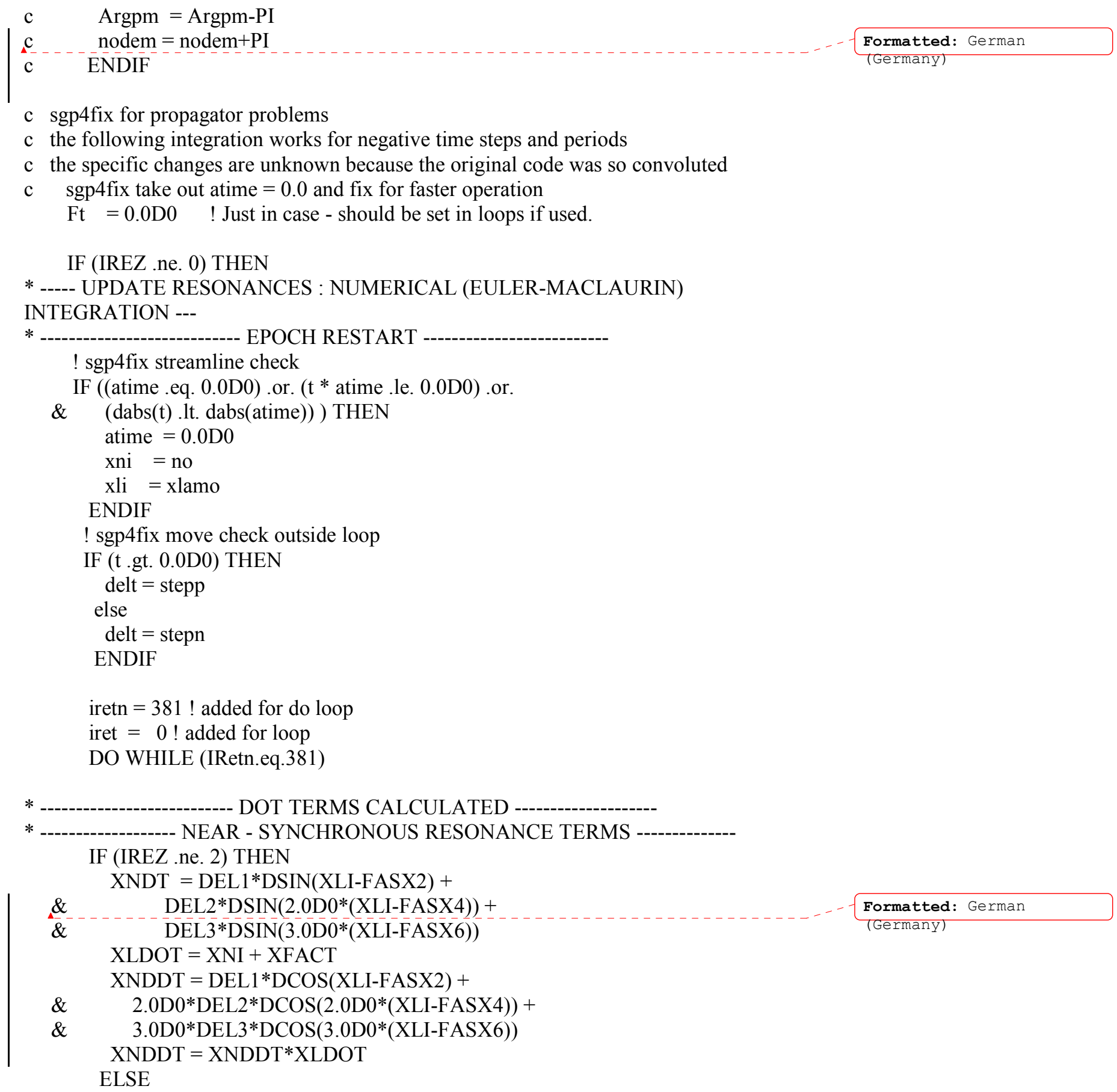




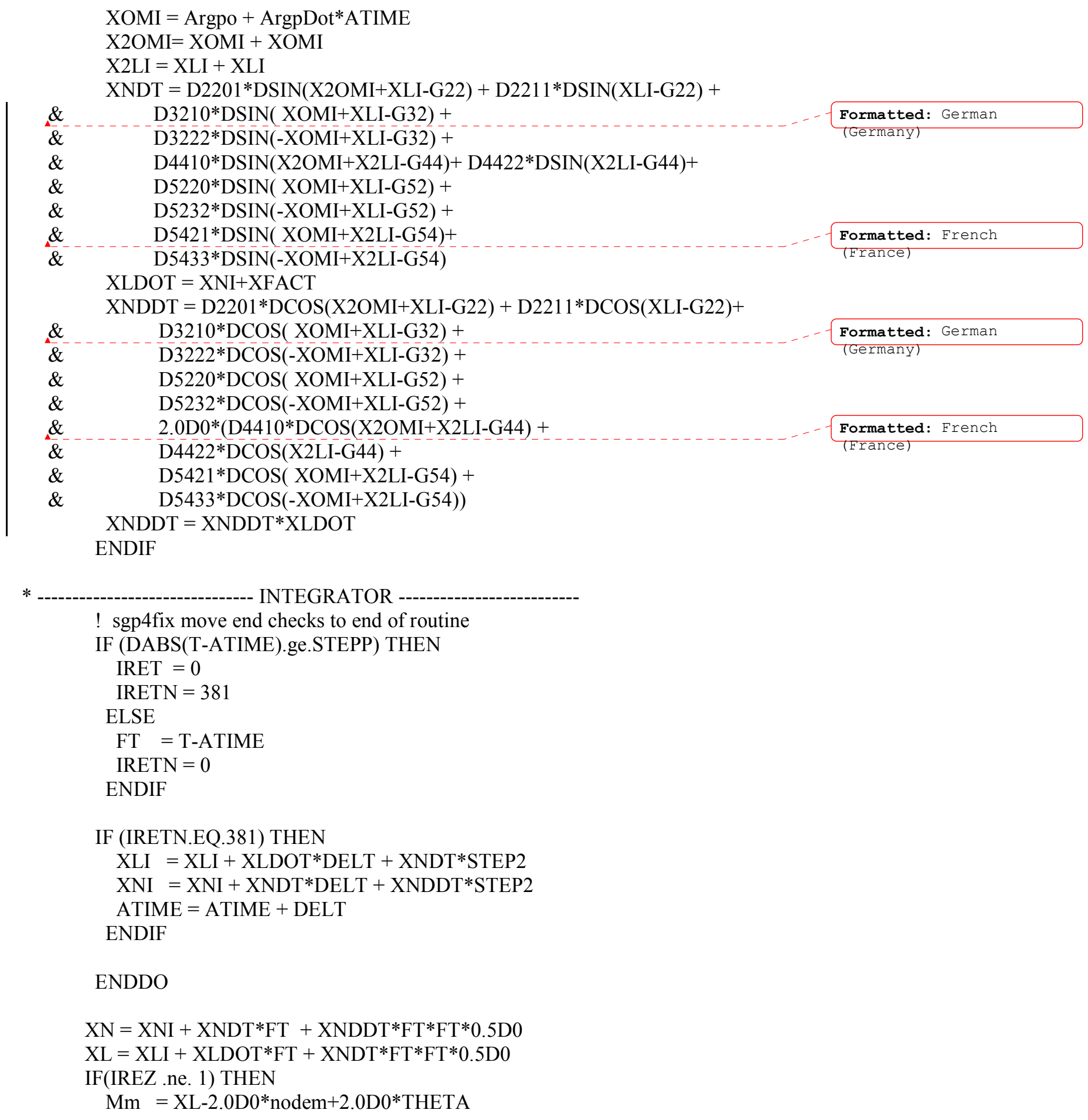




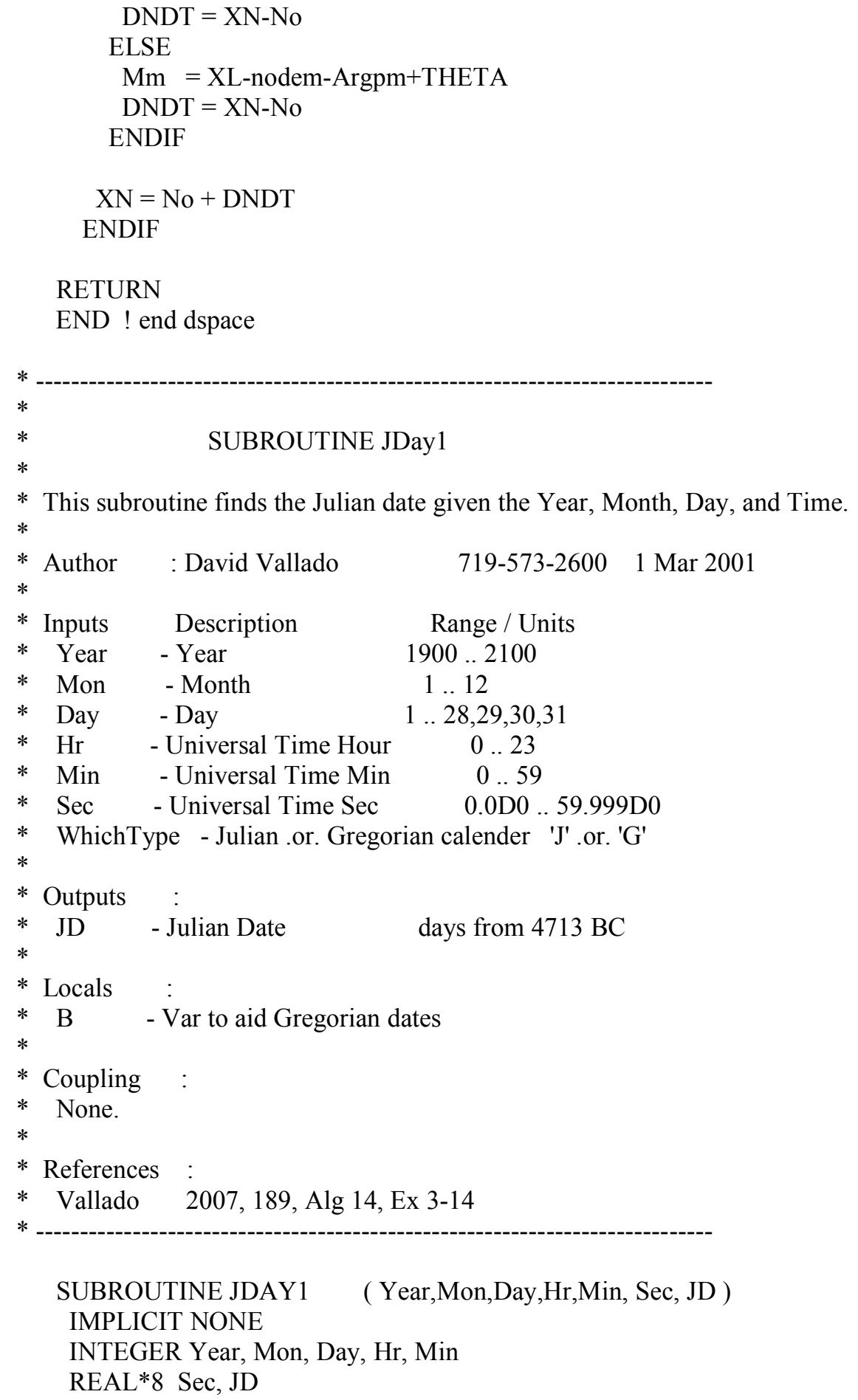




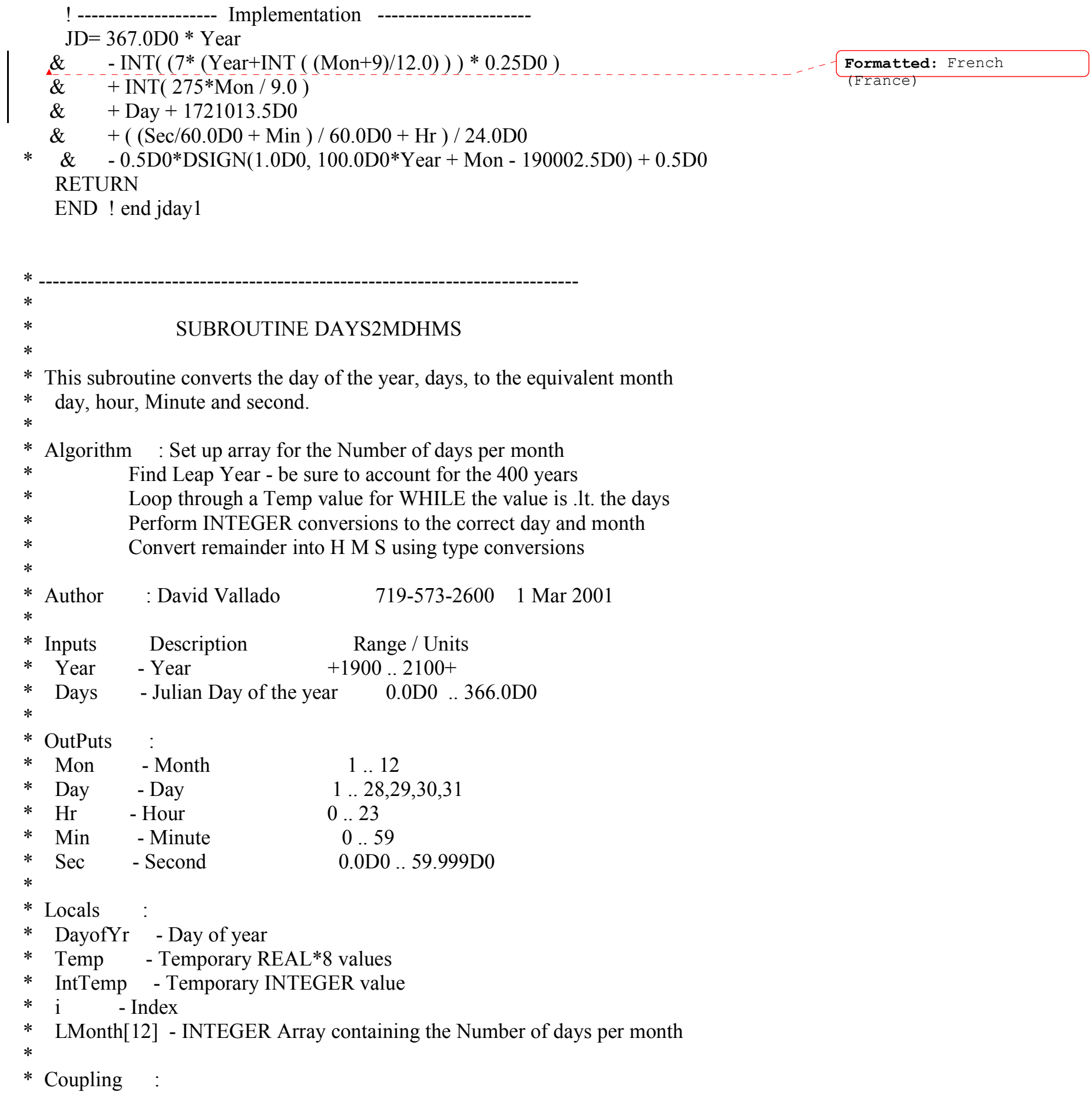


* None.

$*$

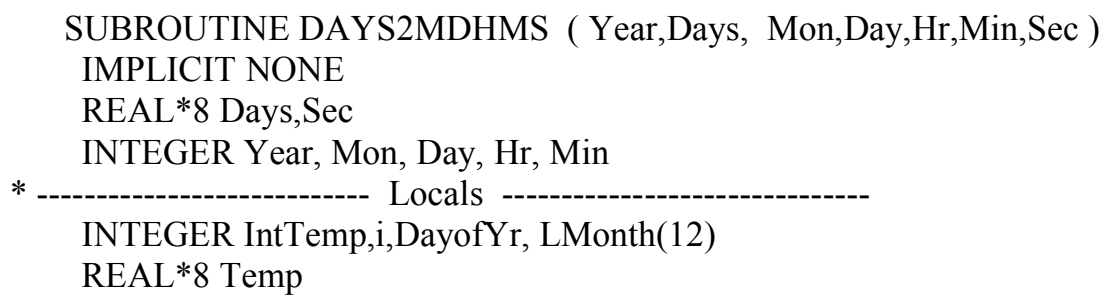

END ! end days $2 \mathrm{mdhms}$ 


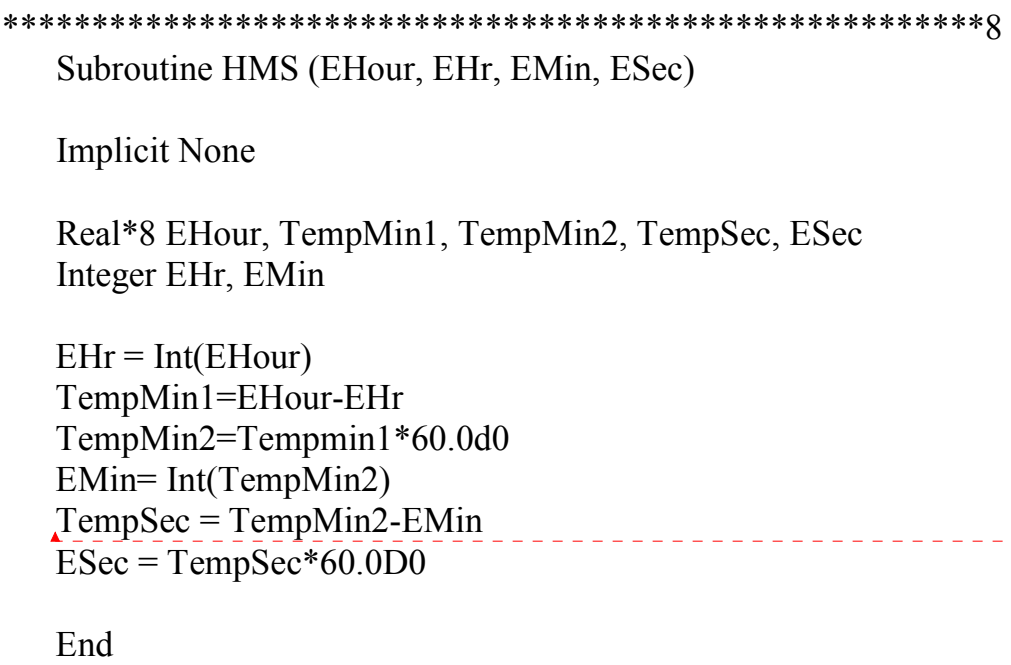

\section{A.2 Other Codes}

Attached on the accompanying CD, there are other codes in their entirety. These include:

- $\quad$ PREDICT (Feb. Version) with updated SGP4 propagator.

- MATLAB version of Vallado’s SGP4 propagator.

- FORTRAN version of Vallado's SGP4 propagator.

Additionally, the analysis files are also provided on the CD. 


\section{APPENDIX B. Verification TLE File}

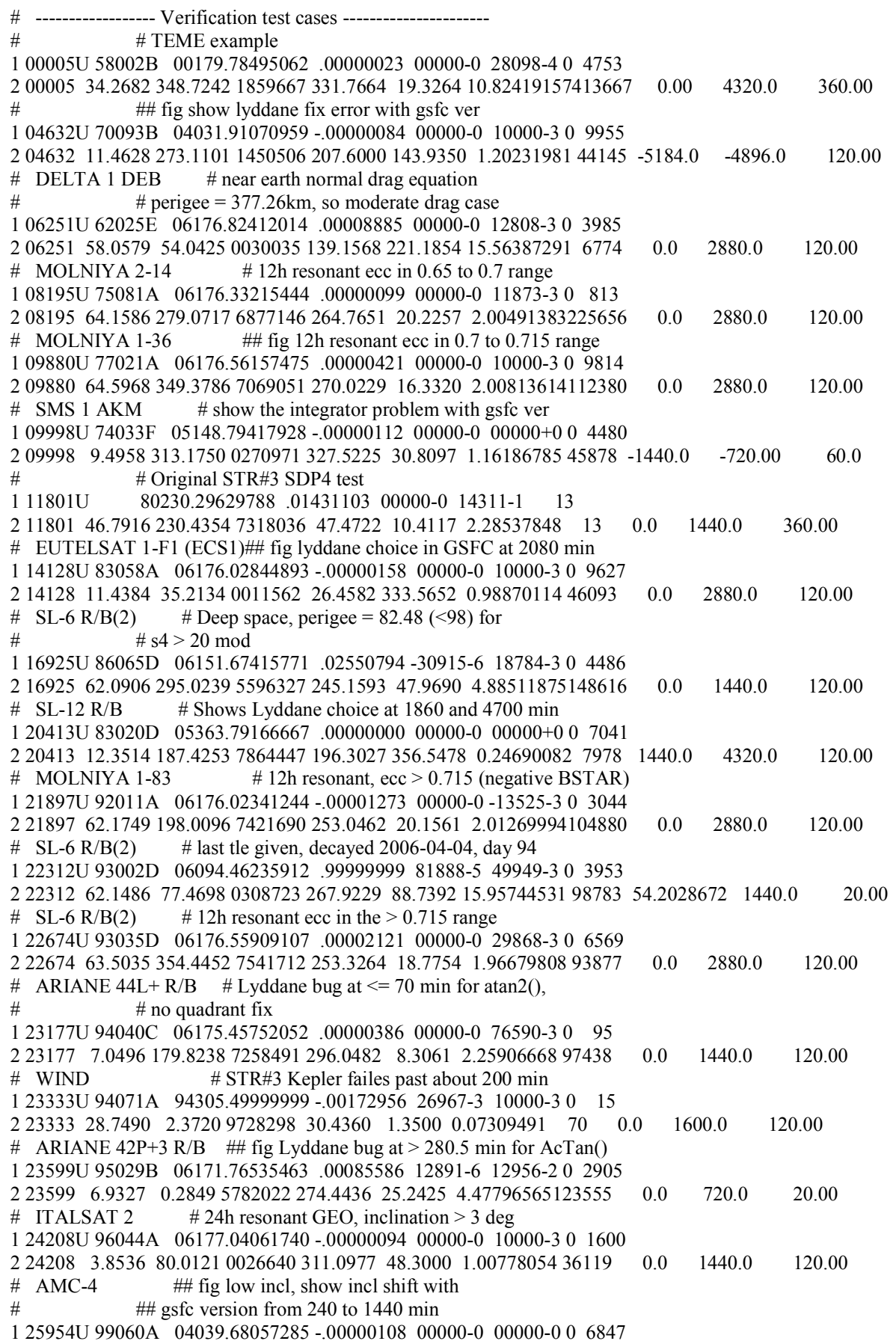




\begin{tabular}{|c|c|c|c|}
\hline \multicolumn{4}{|l|}{$\begin{array}{c}2259540.0004243 .8136000176515 .529422 .713 \\
\text { \# } \quad \text { INTELSAT } 902 \quad \text { \# negative incl at } 9313 \mathrm{~m}\end{array}$} \\
\hline \# \# 270 deg Lyddane bug at $37606 \mathrm{~min}$ & & & \\
\hline \multicolumn{4}{|l|}{1 26900U 01039A $06106.74503247 .00000045 \quad 00000-0 \quad 10000-308290$} \\
\hline $226900 \quad 0.0164266 .5378000331986 .1794182 .2590 \quad 1.0027384716981$ & 9300.00 & 9400.00 & 60.00 \\
\hline \multicolumn{4}{|l|}{ \# COSMOS $1024 \mathrm{DEB} \# 12 \mathrm{~h}$ resonant ecc in 0.5 to 0.65 range } \\
\hline \multicolumn{4}{|l|}{$126975 \mathrm{U} 78066 \mathrm{~F} \quad 06174.85818871 .00000620 \quad 00000-0 \quad 10000-300809$} \\
\hline 22697568.4714236 .13035602877123 .7484302 .57672 .0565755367521 & 0.0 & 2880.0 & 120.00 \\
\hline \multicolumn{4}{|c|}{ \# CBERS $2 \quad$ \# Near Earth, ecc $=8.84 \mathrm{E}-5(<1.0 \mathrm{e}-4)$} \\
\hline \multicolumn{4}{|l|}{ \# \# drop certain normal drag terms } \\
\hline \multicolumn{4}{|l|}{1 28057U 03049A $06177.78615833 .00000060 \quad 00000-0 \quad 35940-4001836$} \\
\hline 22805798.4283247 .6961000088488 .1964271 .932214 .35478080140550 & 0.0 & 2880.0 & 120.00 \\
\hline \\
\hline \multicolumn{4}{|l|}{$128129 \mathrm{U}$ 03058A $\quad 06175.57071136-.00000104 \quad 00000-0 \quad 10000-30 \quad 459$} \\
\hline 22812954.7298324 .80980048506266 .264093 .16632 .0056276818443 & 0.0 & 1440.0 & 120.00 \\
\hline \multicolumn{4}{|l|}{ \# COSMOS $2405 \quad$ \# Near Earth, perigee $=127.20(<156) \mathrm{s} 4 \mathrm{mod}$} \\
\hline \multicolumn{4}{|l|}{1 28350U 04020A $06167.21788666 .16154492 \quad 76267-5 \quad 18678-300884$} \\
\hline & 0.0 & 2880.0 & 120.00 \\
\hline \# H-2 R/B $\quad$ \# Deep space, perigee $=135.75(<156) \mathrm{s} 4 \mathrm{mod}$ & & & \\
\hline \multicolumn{4}{|l|}{128623 U 05006B $\quad 06177.81079184 .00637644 \quad 69054-6 \quad 96390-3 \quad 0 \quad 6000$} \\
\hline 22862328.5200114 .98346249053170 .2550212 .89653 .7947716212753 & 0.0 & 1440.0 & 120.00 \\
\hline \multicolumn{4}{|l|}{ \# XM-3 \# 24h resonant geo, incl $<3$ deg goes } \\
\hline \multicolumn{4}{|l|}{ \# \# negative around $1130 \mathrm{~min}$} \\
\hline \multicolumn{4}{|l|}{1 28626U 05008A $06176.46683397-.00000205 \quad 00000-0 \quad 10000-302190$} \\
\hline \multicolumn{2}{|l|}{$\begin{array}{lllllllll}228626 & 0.0019 & 286.9433 & 0000335 & 13.7918 & 55.6504 & 1.00270176 & 4891 & 0.0\end{array}$} & 1440.0 & 120.00 \\
\hline \multicolumn{4}{|l|}{ \# MINOTAUR R/B＜wide> \# Sub-orbital case - Decayed 2005-11-29 } \\
\hline \multicolumn{4}{|l|}{$\# \quad \#($ perigee $=-51 \mathrm{~km})$, lost in 50 minutes } \\
\hline \multicolumn{4}{|l|}{128872 U 05037B $\quad 05333.02012661 .25992681 \quad 00000-0 \quad 24476-3 \quad 0 \quad 1534$} \\
\hline 22887296.4736157 .99860303955244 .0492110 .652316 .4601593810708 & 0.0 & 50.0 & 5.00 \\
\hline \multicolumn{4}{|l|}{ \# SL-14 DEB \# Last stage of decay - lost in under $420 \mathrm{~min}$} \\
\hline \multicolumn{4}{|l|}{1 29141U 85108AA $06170.26783845 .99999999 \quad 00000-0 \quad 13519-0 \quad 0 \quad 718$} \\
\hline 22914182.4288273 .48820015848277 .212483 .913315 .933430746828 & 0.0 & 440.0 & 20.00 \\
\hline \multicolumn{4}{|l|}{ \# SL-12 DEB $\quad$ \# Near Earth, perigee $=212.24<220$} \\
\hline \multicolumn{4}{|l|}{ \# \# simplified drag eq } \\
\hline $129238 \mathrm{U} 06022 \mathrm{G} \quad 06177.28732010 .00766286 \quad 10823-4 \quad 13334-200101$ & & & \\
\hline 22923851.5595213 .7903020257995 .2503267 .901015 .738238391061 & 0.0 & 1440.0 & 120.00 \\
\hline \# $\quad$ \# Original STR\#3 SGP4 test & & & \\
\hline $\begin{array}{llllllllll}188888 U & 80275.98708465 & .00073094 & 13844-3 & 66816-4 & 0 & 87\end{array}$ & & & \\
\hline $\begin{array}{l}28888872.8435115 .9689008673152 .6988110 .571416 .058245181058 \\
\#\end{array}$ & 0.0 & 1440.0 & 120.00 \\
\hline
\end{tabular}




\title{
APPENDIX C - Verification Results Data
}

\begin{abstract}
$5 \mathrm{xx}$
$\begin{array}{lllllll}0.00000000 & 7022.46529266 & -1400.08296755 & 0.03995155 & 1.893841015 & 6.405893759 & 4.534807250\end{array}$

$360.00000000 \quad-7154.03120202 \quad-3783.17682504 \quad-3536.19412294 \quad 4.741887409-4.151817765-2.093935425$

$720.00000000 \quad-7134.59340119 \quad 6531.68641334 \quad 3260.27186483-4.113793027-2.911922039-2.557327851$

$\begin{array}{llllllll}1080.00000000 & 5568.53901181 & 4492.06992591 & 3863.87641983 & -4.209106476 & 5.159719888 & 2.744852980\end{array}$

$1440.00000000 \quad-938.55923943 \quad-6268.18748831 \quad-4294.02924751 \quad 7.536105209-0.427127707 \quad 0.989878080$

$1800.00000000 \quad-9680.56121728 \quad 2802.47771354 \quad 124.10688038-0.905874102-4.659467970-3.227347517$

$\begin{array}{lllllll}2160.00000000 & 190.19796988 & 7746.96653614 & 5110.00675412 & -6.112325142 & 1.527008184 & -0.139152358\end{array}$

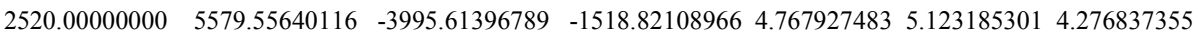

$\begin{array}{lllllll}2880.00000000 & -8650.73082219 & -1914.93811525 & -3007.03603443 & 3.067165127 & -4.828384068 & -2.515322836\end{array}$

$3240.00000000 \quad-5429.79204164 \quad 7574.36493792 \quad 3747.39305236-4.999442110-1.800561422-2.229392830$

$\begin{array}{lllllll}3600.00000000 & 6759.04583722 & 2001.58198220 & 2783.55192533 & -2.180993947 & 6.402085603 & 3.644723952\end{array}$

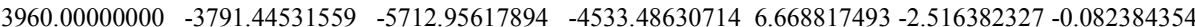

$4320.00000000 \quad-9060.47373569 \quad 4658.70952502 \quad 813.68673153-2.232832783-4.110453490-3.157345433$
\end{abstract}

$4632 \mathrm{xx}$

$\begin{array}{llllllll}0.00000000 & 2334.11450085 & -41920.44035349 & -0.03867437 & 2.826321032 & -0.065091664 & 0.570936053\end{array}$ $\begin{array}{lllllll}-5184.00000000 & -29020.02587128 & 13819.84419063 & -5713.33679183 & -1.768068390 & -3.235371192 & -0.395206135\end{array}$ $\begin{array}{lllllllll}-5064.00000000 & -32982.56870101 & -11125.54996609 & -6803.28472771 & 0.617446996 & -3.379240041 & 0.085954707\end{array}$ $\begin{array}{lllllll}-4944.00000000 & -22097.68730513 & -31583.13829284 & -4836.34329328 & 2.230597499 & -2.166594667 & 0.426443070\end{array}$ $\begin{array}{lllllll}-4896.00000000 & -15129.94694545 & -36907.74526221 & -3487.56256701 & 2.581167187 & -1.524204737 & 0.504805763\end{array}$

$6251 \times x$

$\begin{array}{lllllll}0.00000000 & 3988.31022699 & 5498.96657235 & 0.90055879 & -3.290032738 & 2.357652820 & 6.496623475\end{array}$ $120.00000000 \quad-3935.69800083 \quad 409.10980837 \quad 5471.33577327-3.374784183-6.635211043-1.942056221$ $\begin{array}{lllllll}240.00000000 & -1675.12766915 & -5683.30432352 & -3286.21510937 & 5.282496925 & 1.508674259 & -5.354872978\end{array}$ $\begin{array}{llllllll}360.00000000 & 4993.62642836 & 2890.54969900 & -3600.40145627 & 0.347333429 & 5.707031557 & 5.070699638\end{array}$ $480.00000000 \quad-1115.07959514 \quad 4015.11691491 \quad 5326.99727718-5.524279443-4.7657387742 .402255961$ $\begin{array}{lllllll}600.00000000 & -4329.10008198 & -5176.70287935 & 409.65313857 & 2.858408303 & -2.933091792 & -6.509690397\end{array}$ $\begin{array}{llllllll}720.00000000 & 3692.60030028 & -976.24265255 & -5623.36447493 & 3.897257243 & 6.415554948 & 1.429112190\end{array}$ $840.00000000 \quad 2301.83510037 \quad 5723.92394553 \quad 2814.61514580-5.110924966-0.7645105595 .662120145$

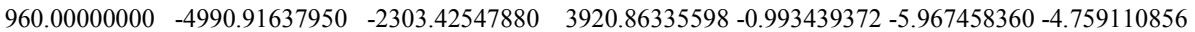
$\begin{array}{llllllll}1080.00000000 & 642.27769977 & -4332.89821901 & -5183.31523910 & 5.720542579 & 4.216573838 & -2.846576139\end{array}$ $1200.00000000 \quad 4719.78335752 \quad 4798.06938996 \quad-943.58851062-2.2948606623 .4924993896 .408334723$ $1320.00000000 \quad-3299.16993602 \quad 1576.83168320 \quad 5678.67840638-4.460347074-6.202025196-0.885874586$

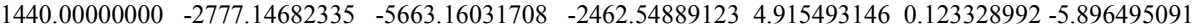
$\begin{array}{llllllll}1560.00000000 & 4992.31573893 & 1716.62356770 & -4287.86065581 & 1.640717189 & 6.071570434 & 4.338797931\end{array}$ $1680.00000000 \quad-8.22384755 \quad 4662.21521668 \quad 4905.66411857-5.891011274-3.593173872 \quad 3.365100460$

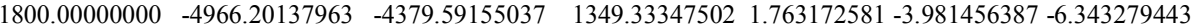
$\begin{array}{llllllll}1920.00000000 & 2954.49390331 & -2080.65984650 & -5754.75038057 & 4.895893306 & 5.858184322 & 0.375474825\end{array}$ $\begin{array}{lllllll}2040.00000000 & 3363.28794321 & 5559.55841180 & 1956.05542266 & -4.587378863 & 0.591943403 & 6.107838605\end{array}$ $2160.00000000 \quad-4856.66780070 \quad-1107.03450192 \quad 4557.21258241-2.304158557-6.186437070-3.956549542$ $\begin{array}{llllllll}2280.00000000 & -497.84480071 & -4863.46005312 & -4700.81211217 & 5.960065407 & 2.996683369 & -3.767123329\end{array}$ $\begin{array}{llllllll}2400.00000000 & 5241.61936096 & 3910.75960683 & -1857.93473952 & -1.124834806 & 4.406213160 & 6.148161299\end{array}$ $\begin{array}{lllllll}2520.00000000 & -2451.38045953 & 2610.60463261 & 5729.79022069 & -5.366560525 & -5.500855666 & 0.187958716\end{array}$

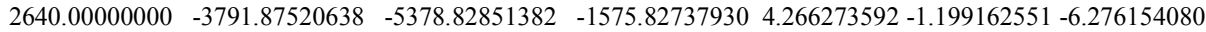
$\begin{array}{llllllll}2760.00000000 & 4730.53958356 & 524.05006433 & -4857.29369725 & 2.918056288 & 6.135412849 & 3.495115636\end{array}$ $\begin{array}{lllllll}2880.00000000 & 1159.27802897 & 5056.60175495 & 4353.49418579 & -5.968060341 & -2.314790406 & 4.230722669\end{array}$

$8195 \mathrm{xx}$

$\begin{array}{lllllll}0.00000000 & 2349.89483350 & -14785.93811562 & 0.02119378 & 2.721488096 & -3.256811655 & 4.498416672\end{array}$ $\begin{array}{llllllll}120.00000000 & 15223.91713658 & -17852.95881713 & 25280.39558224 & 1.079041732 & 0.875187372 & 2.485682813\end{array}$ $\begin{array}{lllllll}240.00000000 & 19752.78050009 & -8600.07130962 & 37522.72921090 & 0.238105279 & 1.546110924 & 0.986410447\end{array}$ 
$360.00000000 \quad 19089.29762968 \quad 3107.89495018 \quad 39958.14661370-0.4103080341 .640332277-0.306873818$ $\begin{array}{lllllll}480.00000000 & 13829.66070574 & 13977.39999817 & 32736.32082508 & -1.065096849 & 1.279983299 & -1.760166075\end{array}$

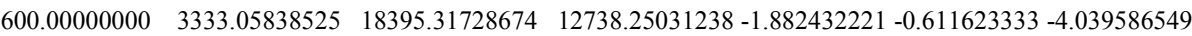
$\begin{array}{lllllll}720.00000000 & 2622.13222207 & -15125.15464924 & 474.51048398 & 2.688287199 & -3.078426664 & 4.494979530\end{array}$

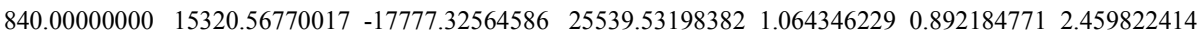
$\begin{array}{llllllll}960.00000000 & 19769.70267785 & -8458.65104454 & 37624.20130236 & 0.229304396 & 1.550363884 & 0.966993056\end{array}$ $\begin{array}{lllllll}1080.00000000 & 19048.56201523 & 3260.43223119 & 39923.39143967 & -0.418015536 & 1.639346953 & -0.326094840\end{array}$ $1200.00000000 \quad 13729.19205837 \quad 14097.70014810 \quad 32547.52799890-1.074511043 \quad 1.270505211-1.785099927$ $1320.00000000 \quad 3148.86165643 \quad 18323.19841703 \quad 12305.75195578-1.895271701-0.678343847-4.086577951$ $\begin{array}{lllllll}1440.00000000 & 2890.80638268 & -15446.43952300 & 948.77010176 & 2.654407490 & -2.909344895 & 4.486437362\end{array}$ $\begin{array}{llllllll}1560.00000000 & 15415.98410712 & -17699.90714437 & 25796.19644689 & 1.049818334 & 0.908822332 & 2.434107329\end{array}$ $\begin{array}{llllllll}1680.00000000 & 19786.00618538 & -8316.74570581 & 37723.74539119 & 0.220539813 & 1.554518900 & 0.947601047\end{array}$ $\begin{array}{llllllll}1800.00000000 & 19007.28688729 & 3412.85948715 & 39886.66579255 & -0.425733568 & 1.638276809 & -0.345353807\end{array}$ $1920.00000000 \quad 13627.93015254 \quad 14216.95401307 \quad 32356.13706868-1.083991976 \quad 1.260802347-1.810193903$ $2040.00000000 \quad 2963.26486560 \quad 18243.85063641 \quad 11868.25797486-1.908015447-0.747870342-4.134004492$ $2160.00000000 \quad 3155.85126036-15750.70393364 \quad 1422.32496953 \quad 2.620085624-2.748990396 \quad 4.473527039$ $2280.00000000 \quad 15510.15191770 \quad-17620.71002219 \quad 26050.43525345 \quad 1.0354546780 .925111006 \quad 2.408534465$ $\begin{array}{lllllllll}2400.00000000 & 19801.67198812 & -8174.33337167 & 37821.38577439 & 0.211812700 & 1.558576937 & 0.928231880\end{array}$ $2520.00000000 \quad 18965.46529379 \quad 3565.19666242 \quad 39847.97510998-0.433459945 \quad 1.637120585-0.364653213$ $2640.00000000 \quad 13525.88227400 \quad 14335.15978787 \quad 32162.13236536-1.093537945 \quad 1.250868256-1.835451681$ $\begin{array}{lllllll}2760.00000000 & 2776.30574260 & 18156.98538451 & 11425.73046481 & -1.920632199 & -0.820370733 & -4.181839232\end{array}$ $2880.00000000 \quad 3417.20931586-16038.79510665 \quad 1894.74934058 \quad 2.585515864-2.596818146 \quad 4.456882556$

$9880 \mathrm{xx}$

$\begin{array}{lllllll}0.00000000 & 13020.06750784 & -2449.07193500 & 1.15896030 & 4.247363935 & 1.597178501 & 4.956708611\end{array}$

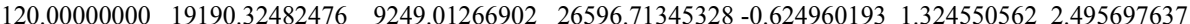
$\begin{array}{lllllll}240.00000000 & 11332.67806218 & 16517.99124008 & 38569.78482991 & -1.400974747 & 0.710947006 & 0.923935636\end{array}$

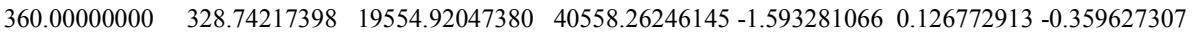
$480.00000000-10684.90590680 \quad 18057.15728839 \quad 33158.75253886-1.383205997-0.582328999-1.744412556$ $600.00000000 \quad-17069.78000550 \quad 9944.86797897 \quad 13885.916490590 .044133354-1.853448464-3.815303117$ $\begin{array}{llllllll}720.00000000 & 13725.09398980 & -2180.70877090 & 863.29684523 & 3.878478111 & 1.656846496 & 4.944867241\end{array}$ $\begin{array}{lllllll}840.00000000 & 19089.63879226 & 9456.29670247 & 27026.79562883 & -0.656614299 & 1.309112636 & 2.449371941\end{array}$ $\begin{array}{llllllll}960.00000000 & 11106.41248373 & 16627.60874079 & 38727.35140296 & -1.409722680 & 0.698582526 & 0.891383535\end{array}$ $\begin{array}{lllllll}1080.00000000 & 72.40958621 & 19575.08054144 & 40492.12544001 & -1.593394604 & 0.113655142 & -0.390556063\end{array}$ $1200.00000000-10905.89252576 \quad 17965.41205111 \quad 32850.07298244-1.371396120-0.601706604-1.782817058$

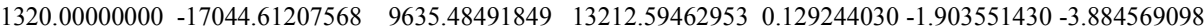
$\begin{array}{llllllll}1440.00000000 & 14369.90303735 & -1903.85601062 & 1722.15319852 & 3.543393116 & 1.701687176 & 4.913881358\end{array}$ $\begin{array}{llllllll}1560.00000000 & 18983.96210441 & 9661.12233804 & 27448.99557732 & -0.687189304 & 1.293808870 & 2.403630759\end{array}$ $1680.00000000 \quad 10878.79336704 \quad 16735.31433954 \quad 38879.23434264-1.418239666 \quad 0.686235750 \quad 0.858951848$ $\begin{array}{lllllll}1800.00000000 & -184.03743100 & 19593.09371709 & 40420.40606889 & -1.593348925 & 0.100448697 & -0.421571993\end{array}$ $1920.00000000-11125.12138631 \quad 17870.19488928 \quad 32534.21521208-1.359116236-0.621413776-1.821629856$

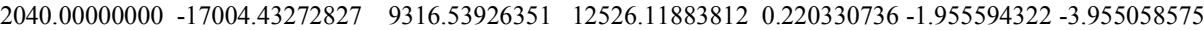
$\begin{array}{llllllll}2160.00000000 & 14960.06492693 & -1620.68430805 & 2574.96359381 & 3.238634028 & 1.734723385 & 4.868880331\end{array}$ $\begin{array}{lllllllll}2280.00000000 & 18873.46347257 & 9863.57004586 & 27863.46574735 & -0.716736981 & 1.278632817 & 2.358448535\end{array}$ $\begin{array}{lllllll}2400.00000000 & 10649.86857581 & 16841.14172669 & 39025.48035006 & -1.426527152 & 0.673901057 & 0.826632332\end{array}$ $\begin{array}{llllllll}2520.00000000 & -440.53459323 & 19608.95524423 & 40343.10675451 & -1.593138597 & 0.087147884 & -0.452680559\end{array}$ $2640.00000000 \quad-11342.45028909 \quad 17771.44223942 \quad 32211.12535721-1.346344015-0.641464291-1.860864234$ $\begin{array}{lllllll}2760.00000000 & -16948.06005711 & 8987.64254880 & 11826.28284367 & 0.318007297 & -2.009693492 & -4.026726648\end{array}$ $2880.00000000 \quad 15500.53445068 \quad-1332.90981042 \quad 3419.72315308 \quad 2.960917974 \quad 1.758331634 \quad 4.813698638$

$9998 \times x$

$\begin{array}{lllllll}0.00000000 & 25532.98947267 & -27244.26327953 & -1.11572421 & 2.410283885 & 2.194175683 & 0.545888526\end{array}$ $\begin{array}{lllllll}-1440.00000000 & -11362.18265118 & -35117.55867813 & -5413.62537994 & 3.137861261 & -1.011678260 & 0.267510059\end{array}$ $\begin{array}{lllllll}-1380.00000000 & 309.25349929 & -36960.43090143 & -4198.48007670 & 3.292429375 & -0.002166046 & 0.402111628\end{array}$ $\begin{array}{llllllll}-1320.00000000 & 11949.04009077 & -35127.37816804 & -2565.89806468 & 3.119942784 & 1.012096444 & 0.497284100\end{array}$ $\begin{array}{lllllll}-1260.00000000 & 22400.45329336 & -29798.63236321 & -677.91515122 & 2.638533344 & 1.922477736 & 0.542792913\end{array}$ $\begin{array}{llllllll}-1200.00000000 & 30640.84752458 & -21525.02340201 & 1277.34808722 & 1.903464941 & 2.634294312 & 0.534540934\end{array}$ $\begin{array}{lllllll}-1140.00000000 & 35899.56788035 & -11152.71158138 & 3108.72535238 & 0.997393045 & 3.079858548 & 0.474873291\end{array}$ $\begin{array}{llllllll}-1080.00000000 & 37732.45438600 & 288.18821054 & 4643.87587495 & 0.016652226 & 3.225184410 & 0.371669746\end{array}$ $\begin{array}{lllllll}-1020.00000000 & 36045.92961699 & 11706.61816230 & 5746.32646574 & -0.942409065 & 3.069888941 & 0.236662980\end{array}$ 
$\begin{array}{lllllll}-960.00000000 & 31076.77273609 & 22063.44379776 & 6325.93403705 & -1.794027976 & 2.642072476 & 0.083556127\end{array}$ $\begin{array}{lllllll}-900.00000000 & 23341.26015320 & 30460.88002531 & 6342.91707895 & -2.469409743 & 1.990861658 & -0.073612096\end{array}$ $\begin{array}{lllllll}-840.00000000 & 13568.39733054 & 36204.45930900 & 5806.79548733 & -2.919354203 & 1.178920217 & -0.221646814\end{array}$ $\begin{array}{lllllll}-780.00000000 & 2628.58762420 & 38840.10855897 & 4771.91979854-3.114400514 & 0.276239109 & -0.348926401\end{array}$

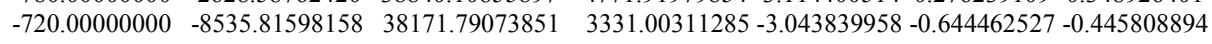

$11801 \mathrm{xx}$

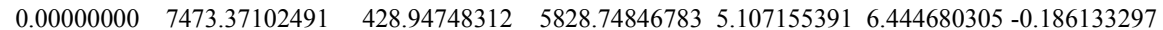
$360.00000000-3305.22148694 \quad 32410.84323331-24697.16974954-1.301137319-1.151315600-0.283335823$ $\begin{array}{lllllll}720.00000000 & 14271.29083858 & 24110.44309009 & -4725.76320143 & -0.320504528 & 2.679841539 & -2.084054355\end{array}$ $\begin{array}{llllllll}1080.00000000 & -9990.05800009 & 22717.34212448 & -23616.88515553 & -1.016674392 & -2.290267981 & 0.728923337\end{array}$

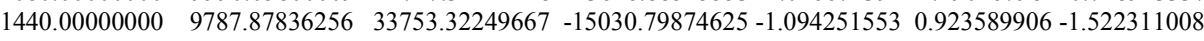

$14128 \mathrm{xx}$

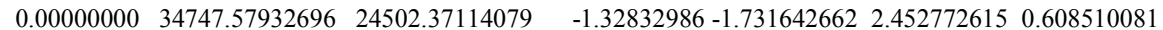
$\begin{array}{lllllll}120.00000000 & 18263.33439094 & 38159.96004751 & 4186.18304085 & -2.744396611 & 1.255583260 & 0.528558932\end{array}$ $\begin{array}{lllllll}240.00000000 & -3023.38840703 & 41783.13186459 & 7273.03412906 & -3.035574793 & -0.271656544 & 0.309645251\end{array}$ $\begin{array}{llllllll}360.00000000 & -23516.34391907 & 34424.42065671 & 8448.49867693 & -2.529120477 & -1.726186020 & 0.009582303\end{array}$

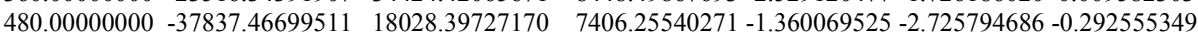
$\begin{array}{llllllll}600.00000000 & -42243.58460661 & -3093.72887774 & 4422.91711801 & 0.163110919 & -3.009980598 & -0.517584362\end{array}$

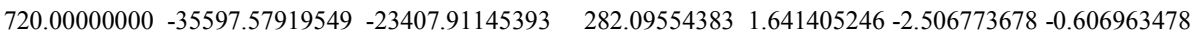
$840.00000000-19649.19834455-37606.11623860 \quad-3932.71525948 \quad 2.689647056-1.349150016-0.537710698$ $\begin{array}{llllllll}960.00000000 & 1431.30912160 & -41982.04949668 & -7120.45467057 & 3.035263353 & 0.160882945 & -0.327993994\end{array}$ $\begin{array}{llllllll}1080.00000000 & 22136.97605384 & -35388.19823762 & -8447.62393401 & 2.587624889 & 1.630097136 & -0.032349004\end{array}$ $\begin{array}{llllllll}1200.00000000 & 37050.15790219 & -19537.23321425 & -7564.83463543 & 1.461844494 & 2.674654256 & 0.272202191\end{array}$ $\begin{array}{llllllll}1320.00000000 & 42253.81760945 & 1431.81867593 & -4699.87621174 & -0.049247334 & 3.019518960 & 0.505890058\end{array}$ $\begin{array}{lllllll}1440.00000000 & 36366.59147396 & 22023.54245720 & -601.47121821 & -1.549681546 & 2.571788981 & 0.607057418\end{array}$ $\begin{array}{llllllll}1560.00000000 & 20922.12287985 & 36826.33975981 & 3654.91125886 & -2.644070068 & 1.447521216 & 0.548722983\end{array}$ $\begin{array}{lllllll}1680.00000000 & -23.77224182 & 41945.51688402 & 6950.29891751 & -3.043358385 & -0.057417440 & 0.346112094\end{array}$ $\begin{array}{llllll}1800.00000000 & -20964.17821076 & 36039.06206172 & 8418.91984963-2.642795221 & -1.546099886 & 0.052725852\end{array}$ $1920.00000000-36401.63863057 \quad 20669.75286162 \quad 7677.19769359-1.549488154-2.627052310-0.254079652$ $2040.00000000-42298.30327543 \quad-119.03351118 \quad 4922.96388841-0.052232768-3.018152669-0.493827331$ $\begin{array}{lllllll}2160.00000000 & -37125.62383511 & -20879.63058368 & 879.86971348 & 1.456499841 & -2.619358421 & -0.604081694\end{array}$ $2280.00000000 \quad-22250.12320553 \quad-36182.74736487 \quad-3393.15365183 \quad 2.583161226-1.536647628-0.556404555$ $2400.00000000-1563.06258654-42035.43179159-6780.021617603 .034917506-0.052702046-0.363395654$ $\begin{array}{llllllll}2520.00000000 & 19531.64069587 & -36905.65470956 & -8395.46892032 & 2.693682199 & 1.446079999 & -0.075256054\end{array}$ $\begin{array}{llllllll}2640.00000000 & 35516.53506142 & -22123.71916638 & -7815.04516935 & 1.646882125 & 2.568416058 & 0.232985912\end{array}$ $\begin{array}{llllllll}2760.00000000 & 42196.03535976 & -1547.32646751 & -5187.39401981 & 0.166491841 & 3.019211549 & 0.480665780\end{array}$ $\begin{array}{lllllll}2880.00000000 & 37802.25393045 & 19433.57330019 & -1198.66634226 & -1.359930580 & 2.677830903 & 0.602507466\end{array}$

$16925 \mathrm{xx}$

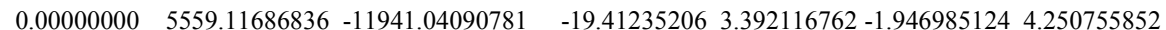
$\begin{array}{lllllll}120.00000000 & 12339.83273749 & -2771.14447871 & 18904.57603433 & -0.871247614 & 2.600917693 & 0.581560002\end{array}$ $240.00000000 \quad-3385.00215658 \quad 7538.13955729 \quad 200.59008616-2.023512865-4.261808344-6.856385787$ $\begin{array}{lllllll}360.00000000 & 12805.22442200 & -10258.94667177 & 13780.16486738 & 0.619279224 & 1.821510542 & 2.507365975\end{array}$ $\begin{array}{lllllll}480.00000000 & 5682.46556318 & 7199.30270473 & 15437.67134070 & -2.474365406 & 2.087897336 & -2.583767460\end{array}$ $\begin{array}{llllllll}600.00000000 & 7628.94243982 & -12852.72097492 & 2902.87208981 & 2.748131081 & -0.740084579 & 4.125307943\end{array}$ $\begin{array}{llllllll}720.00000000 & 11531.64866625 & -858.27542736 & 19086.85993771 & -1.170071901 & 2.660311986 & 0.096005705\end{array}$ $\begin{array}{lllllll}840.00000000 & -3866.98069515 & 2603.73442786 & -4577.36484577 & 1.157257298 & -8.453281164 & -4.683959407\end{array}$ $\begin{array}{llllllll}960.00000000 & 13054.77732721 & -8707.92757730 & 15537.63259903 & 0.229846748 & 2.119467054 & 2.063396852\end{array}$ $\begin{array}{llllllll}1080.00000000 & 3496.91064652 & 8712.83919778 & 12845.81838327 & -2.782184997 & 1.552950644 & -3.554436131\end{array}$ $\begin{array}{llllllll}1200.00000000 & 9593.07424729 & -13023.75963608 & 6250.46484931 & 2.072666376 & 0.278735334 & 3.778111073\end{array}$ $1320.00000000 \quad 10284.79205084 \quad 1487.89914169 \quad 18824.37381327-1.530335053 \quad 2.663107730-0.542205966$ $\begin{array}{llllllll}1440.00000000 & -984.62035146 & -5187.03480813 & -5745.59594144 & 4.340271916 & -7.266811354 & 1.777668888\end{array}$

$20413 \mathrm{xx}$

$\begin{array}{lllllll}0.00000000 & 25123.29290741 & -13225.49966287 & 3249.40351869 & 0.488683419 & 4.797897593 & -0.961119693\end{array}$ $1440.00000000-151669.05280515 \quad-5645.20454550 \quad-2198.51592118-0.869182889-0.8707598720 .156508219$ 
$1560.00000000-157497.71657495-11884.99595074-1061.44439402-0.749657961-0.8640167150 .157766101$ $1680.00000000-162498.32255577-18062.99733167 \quad 81.00915253-0.638980378-0.853687105 \quad 0.158098992$

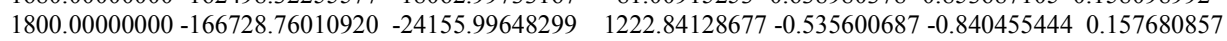
$1920.00000000-169935.81924592-31767.29787964 \quad 2749.01540345-0.430050431-0.828904183 \quad 0.157812340$ $2040.00000000-172703.07831815-37662.95639336 \quad 3883.60052579-0.338004891-0.810277487 \quad 0.156020035$ $2160.00000000-174823.19337404-43417.55605219 \quad 5003.26312809-0.250258622-0.7898286720 .153764903$ $2280.00000000-176324.63925775-49018.51958648 \quad 6104.85025002-0.166136613-0.7677062620 .151092242$ $2400.00000000-177231.42142458-54454.12699497 \quad 7185.48661607-0.085067854-0.744001567 \quad 0.148033403$ $2520.00000000-177563.73583232-59713.14859144 \quad 8242.48472591-0.006561730-0.718760309 \quad 0.144608676$ $2640.00000000-177338.48026483-64784.54644698 \quad 9273.27220003 \quad 0.069809946-0.691990238 \quad 0.140829236$ $\begin{array}{lllllll}2760.00000000 & -176569.65151461 & -69657.21976255 & 10275.33063459 & 0.144426878 & -0.663665876 & 0.136698419\end{array}$ $2880.00000000-175268.65299073-74319.77625463 \quad 11246.14177160 \quad 0.217631370-0.633731091 \quad 0.132212491$ $3000.00000000-173444.53039609-78760.31560396 \quad 12183.137752120 .289737325-0.6020999290 .127361017$

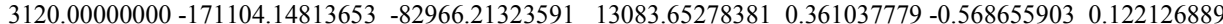
$3240.00000000-168252.31543803 \quad-86923.89363433 \quad 13944.87382716 \quad 0.431811396-0.533249797 \quad 0.116486022$ $\begin{array}{lllllll}3360.00000000 & -164891.86832887 & -90618.58225954 & 14763.78794247 & 0.502328269 & -0.495695896 & 0.110406725\end{array}$ $3480.00000000-161023.71139825-94034.02398835 \quad 15537.123757290 .572855321-0.455766412 \quad 0.103848688$ $\begin{array}{lllllll}3600.00000000 & -156646.82136725 & -97152.15370791 & 16261.28409305 & 0.643661538 & -0.413183688 & 0.096761524\end{array}$ $3720.00000000-151758.21285737-99952.70098346 \quad 16932.26607548 \quad 0.715023254-0.367609561 \quad 0.089082727$ $3840.00000000-146352.86521283-102412.70506284 \quad 17545.56394158 \quad 0.787229695-0.318630913 \quad 0.080734873$ $\begin{array}{lllllll}3960.00000000 & -140423.60777444 & -104505.90799734 & 18096.04807097 & 0.860588979 & -0.265739987 & 0.071621768\end{array}$ $4080.00000000-133960.95961851-106201.98091318 \quad 18577.81121953 \quad 0.935434758-0.208307307 \quad 0.061623110$ $4200.00000000-126952.91860010-107465.51906186 \quad 18983.96903112 \quad 1.012133628-0.145543878 \quad 0.050587007$ $4320.00000000-119384.69396454-108254.71115372 \quad 19306.39581892 \quad 1.091093313-0.0764474790 .038319282$

$21897 \mathrm{xx}$

$0.00000000-14464.72135182-4699.19517587 \quad 0.06681686-3.249312013-3.2810327074 .007046940$ $120.00000000-19410.46286123-19143.03318969 \quad 23114.055226190 .508602237-1.1568822692 .379923455$ $\begin{array}{llllllll}240.00000000 & -12686.06129708 & -23853.75335645 & 35529.81733588 & 1.231633829 & -0.221718202 & 1.118440291\end{array}$ $\begin{array}{llllllll}360.00000000 & -2775.46649359 & -22839.64574119 & 39494.64689967 & 1.468963405 & 0.489481769 & -0.023972788\end{array}$ $\begin{array}{llllllll}480.00000000 & 7679.87883570 & -16780.50760106 & 34686.21815555 & 1.364171080 & 1.211183897 & -1.385151371\end{array}$

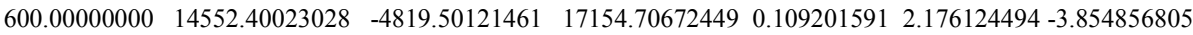
$720.00000000-15302.38845375 \quad-5556.43440300 \quad 1095.95088753-2.838224312-3.134231137 \quad 3.992596326$ $840.00000000-19289.20066748 \quad-19427.04851118 \quad 23759.45685636 \quad 0.552495087-1.112499437 \quad 2.325112654$ $960.00000000-12376.21976437-23893.38020018 \quad 35831.33691892 \quad 1.246701529-0.194294048 \quad 1.074867282$

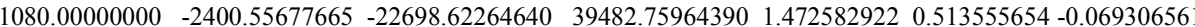
$\begin{array}{llllllll}1200.00000000 & 8031.66819252 & -16455.77592085 & 34298.94391742 & 1.351357426 & 1.239633234 & -1.448195324\end{array}$ $\begin{array}{llllllll}1320.00000000 & 14559.48780372 & -4238.43773813 & 16079.23154704 & -0.026409655 & 2.218938770 & -4.012628896\end{array}$ $1440.00000000-16036.04980660-6372.51406468 \quad 2183.44834232-2.485113443-2.994994355 \quad 3.955891272$ $1560.00000000-19156.71583814-19698.89059957 \quad 24389.29473934 \quad 0.594278133-1.0694185992 .271152044$ $1680.00000000-12062.72925552-23925.82362911 \quad 36120.66680667 \quad 1.261238798-0.167201856 \quad 1.031478939$ $\begin{array}{llllllll}1800.00000000 & -2024.96136966 & -22551.56626703 & 39458.50085787 & 1.475816889 & 0.537615764 & -0.114887472\end{array}$

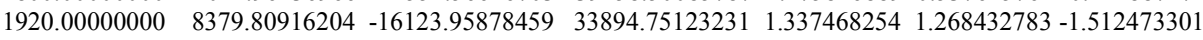
$2040.00000000 \quad 14527.86748873 \quad-3646.33817120 \quad 14960.74306518-0.1800358392 .261273515-4.179355590$ $2160.00000000-16680.12147335 \quad-7149.80800425 \quad 3257.64227208-2.178897351-2.863927095 \quad 3.904876943$ $2280.00000000-19013.58793448-19958.93766022 \quad 25003.81778666 \quad 0.634100431-1.0275598232 .218002685$ $2400.00000000-11745.76155818-23951.19438627 \quad 36397.87676581 \quad 1.275261813-0.1404251320 .988259441$

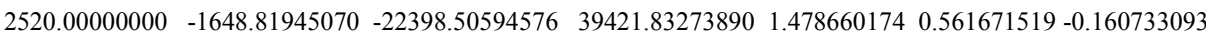
$\begin{array}{llllllll}2640.00000000 & 8723.97652795 & -15784.99406275 & 33473.35215527 & 1.322433593 & 1.297602497 & -1.578055493\end{array}$ $2760.00000000 \quad 14452.25571587 \quad-3043.42332645 \quad 13796.84870805-0.3551901692 .302485443-4.355767077$ $2880.00000000-17246.31075678 \quad-7890.72601508 \quad 4315.39410307-1.910968458-2.740945672 \quad 3.844722726$

$22312 \mathrm{xx}$

$\begin{array}{lllllll}0.00000000 & 1442.10132912 & 6510.23625449 & 8.83145885 & -3.475714837 & 0.997262768 & 6.835860345\end{array}$ $\begin{array}{lllllll}54.20286720 & 306.10478453 & -5816.45655525 & -2979.55846068 & 3.950663855 & 3.415332543 & -5.879974329\end{array}$ $\begin{array}{lllllll}74.20286720 & 3282.82085464 & 2077.46972905 & -5189.17988770 & 0.097342701 & 7.375135692 & 2.900196702\end{array}$ $\begin{array}{lllllll}94.20286720 & 530.82729176 & 6426.20790003 & 1712.37076793 & -3.837120395 & -1.252430637 & 6.561602577\end{array}$

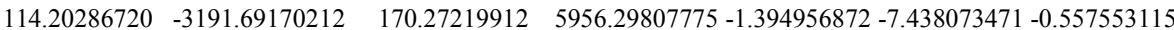
$134.20286720 \quad-1818.99222465 \quad-6322.45146616 \quad 681.952471543 .349795173-1.530140265-6.831522765$ $\begin{array}{lllllll}154.20286720 & 2515.66448634 & -2158.83091224 & -5552.13320544 & 2.571979660 & 7.311930509 & -1.639865620\end{array}$ 
$\begin{array}{llllllll}174.20286720 & 2414.52833210 & 5749.10150922 & -1998.59693165 & -2.681032960 & 3.527589301 & 6.452951429\end{array}$ $\begin{array}{llllllll}194.20286720 & -1877.98944331 & 3862.27848302 & 5112.48435863 & -3.261489804 & -6.026859137 & 3.433254768\end{array}$ $\begin{array}{lllllll}214.20286720 & -3117.36584395 & -4419.74773864 & 3840.85960912 & 1.545479182 & -5.475416581 & -5.207913748\end{array}$ $\begin{array}{llllllll}234.20286720 & 815.32034678 & -5231.67692249 & -3760.04690354 & 3.870864200 & 4.455588552 & -5.211082191\end{array}$ $\begin{array}{llllllll}254.20286720 & 3269.54341810 & 3029.00081083 & -4704.67969713 & -0.526711345 & 6.812157950 & 3.929825087\end{array}$

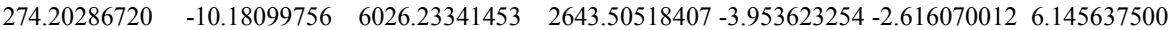
$294.20286720 \quad-3320.58819584 \quad-1248.42679945 \quad 5563.06017927-0.637046974-7.417786044-2.076120187$ $\begin{array}{lllllll}314.20286720 & -1025.48974616 & -6366.98945782 & -911.23559153 & 3.811771909 & 0.438071490 & -6.829260617\end{array}$ $\begin{array}{llllllll}334.20286720 & 3003.75996128 & -413.85708003 & -5706.15591435 & 1.674350083 & 7.694169068 & 0.316915204\end{array}$ $\begin{array}{lllllll}354.20286720 & 1731.42816980 & 6258.27676925 & -409.32527982 & -3.400497806 & 1.447945424 & 6.904010052\end{array}$ $\begin{array}{lllllll}374.20286720 & -2582.52111460 & 2024.19020680 & 5647.55650268 & -2.530348121 & -7.221719393 & 1.438141553\end{array}$ $\begin{array}{llllllll}394.20286720 & -2440.56848578 & -5702.77311877 & 1934.81094689 & 2.731792947 & -3.350576075 & -6.527773339\end{array}$ $\begin{array}{llllllll}414.20286720 & 1951.22934391 & -3423.59443045 & -5121.67808201 & 3.249039133 & 6.465974362 & -3.069806659\end{array}$ $\begin{array}{llllllll}434.20286720 & 2886.50939356 & 4888.68626216 & -3096.29885989 & -1.973162139 & 4.877039020 & 5.832414910\end{array}$ $\begin{array}{lllllll}454.20286720 & -1276.55532182 & 4553.26898463 & 4406.19787375 & -3.715146421 & -5.320176914 & 4.418210777\end{array}$ $\begin{array}{lllllll}474.20286720 & -3181.54698042 & -3831.29976506 & 4096.80242787 & 1.114159970 & -6.104773578 & -4.829967400\end{array}$

$22674 \mathrm{xx}$

$\begin{array}{llllllll}0.00000000 & 14712.22023280 & -1443.81061850 & 0.83497888 & 4.418965470 & 1.629592098 & 4.115531802\end{array}$ $\begin{array}{lllllll}120.00000000 & 25418.88807860 & 9342.60307989 & 23611.46690798 & 0.051284086 & 1.213127306 & 2.429004159\end{array}$ $\begin{array}{llllllll}240.00000000 & 21619.59550749 & 16125.24978864 & 36396.79365831 & -0.963604380 & 0.685454965 & 1.177181937\end{array}$ $\begin{array}{llllllll}360.00000000 & 12721.50543331 & 19258.96193362 & 40898.47648359 & -1.457448565 & 0.179955469 & 0.071502601\end{array}$ $480.00000000 \quad 1272.80760054 \quad 18458.41971897 \quad 37044.74742696-1.674863386-0.436454983-1.201040990$ $600.00000000-10058.43188619 \quad 11906.60764454 \quad 21739.62097733-1.245829683-1.543789125-3.324449221$ $\begin{array}{llllllll}720.00000000 & 10924.40116466 & -2571.92414170 & -2956.34856294 & 6.071727751 & 1.349579102 & 3.898430260\end{array}$

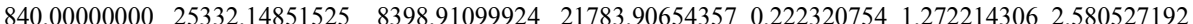
$\begin{array}{llllllll}960.00000000 & 22317.71926039 & 15574.82086129 & 35495.77144092 & -0.892750056 & 0.737383381 & 1.291738834\end{array}$

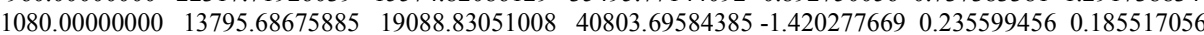
$\begin{array}{lllll}1200.00000000 & 2515.17145049 & 18746.63776282 & 37864.58088636-1.668016053 & -0.360431458-1.052854596\end{array}$ $1320.00000000 \quad-9084.48602106 \quad 12982.62608646 \quad 24045.63900249-1.378032363-1.373184736-3.013963835$

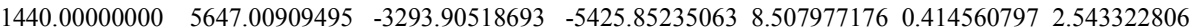
$\begin{array}{llllllll}1560.00000000 & 25111.63372210 & 7412.55109488 & 19844.25781729 & 0.416496290 & 1.332106006 & 2.739301737\end{array}$ $\begin{array}{lllllllll}1680.00000000 & 22961.47461641 & 14985.74459578 & 34511.09257381 & -0.816711048 & 0.789391108 & 1.407901804\end{array}$ $\begin{array}{llllllll}1800.00000000 & 14841.15301459 & 18876.91439870 & 40626.25901619 & -1.380403341 & 0.290228810 & 0.298258120\end{array}$ $\begin{array}{lllllll}1920.00000000 & 3750.70174081 & 18978.57939698 & 38578.11783220 & -1.656939412 & -0.287930881 & -0.910825599\end{array}$ $2040.00000000 \quad-8027.30219489 \quad 13939.54436955 \quad 26136.49045637-1.474476061-1.222693624-2.737178731$ $2160.00000000 \quad-1296.95657092 \quad-2813.69369768 \quad-5871.09587258 \quad 9.881929371-1.978467207-1.922261005$ $\begin{array}{lllllll}2280.00000000 & 24738.60364819 & 6383.41644019 & 17787.27631900 & 0.639556952 & 1.392554379 & 2.906206324\end{array}$ $\begin{array}{lllllllll}2400.00000000 & 23546.85388669 & 14358.15602832 & 33441.67679479 & -0.734895006 & 0.841564851 & 1.526009909\end{array}$ $\begin{array}{llllllll}2520.00000000 & 15855.87696303 & 18624.05633582 & 40367.13420574 & -1.337753546 & 0.343969522 & 0.410018472\end{array}$ $2640.00000000 \quad 4976.44933591 \quad 19156.75504042 \quad 39189.68603184-1.642084365-0.218525096-0.774148204$ $2760.00000000-6909.20746210 \quad 14790.44707042 \quad 28034.46732222-1.545152610-1.088119523-2.487447214$

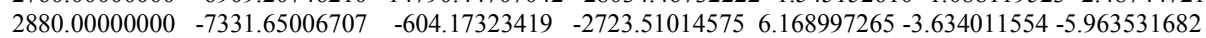

$23177 \mathrm{xx}$

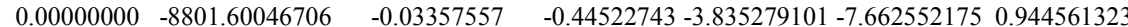
$\begin{array}{lllllll}120.00000000 & -1684.34352858 & -31555.95196340 & 3888.99944319 & 2.023055719 & -2.151306405 & 0.265065778\end{array}$ $240.00000000 \quad 12325.51410155-38982.15046244 \quad 4802.88832275 \quad 1.763224157-0.102514446 \quad 0.012397139$ $\begin{array}{llllllll}360.00000000 & 22773.66831936 & -34348.02176606 & 4228.77407391 & 1.067616787 & 1.352427865 & -0.166956367\end{array}$ $\begin{array}{lllllll}480.00000000 & 26194.40441089 & -19482.94203672 & 2393.84774063 & -0.313732186 & 2.808771328 & -0.346204118\end{array}$ $\begin{array}{llllllll}600.00000000 & 8893.50573448 & 5763.38890561 & -713.69884164-7.037399220 & 3.022613131 & -0.370272416\end{array}$ $720.00000000 \quad-6028.75686537 \quad-25648.99913786 \quad 3164.37107274 \quad 1.883159288-3.177051976 \quad 0.390793162$ $840.00000000 \quad 8313.57299056 \quad-38146.45710922 \quad 4697.80777535 \quad 1.905002133-0.625883074 \quad 0.076098187$ $\begin{array}{lllllll}960.00000000 & 20181.29108622 & -36842.60674073 & 4529.12568218 & 1.326244476 & 0.921916487 & -0.114527455\end{array}$ $\begin{array}{lllllll}1080.00000000 & 26302.61794569 & -25173.39539436 & 3084.65309986 & 0.245398835 & 2.329974347 & -0.287495880\end{array}$ $1200.00000000 \quad 19365.07045602 \quad-2700.00490122 \quad 317.42727417-3.0097330183 .902496058-0.478928582$ $1320.00000000 \quad-9667.81878780-16930.19112642 \quad 2095.87469034 \quad 1.279288285-4.736005905 \quad 0.582878255$ $\begin{array}{lllllll}1440.00000000 & 4021.31438583 & -36066.09209609 & 4442.91587411 & 2.007322354 & -1.227461376 & 0.149383897\end{array}$

$23333 \mathrm{xx}$ 


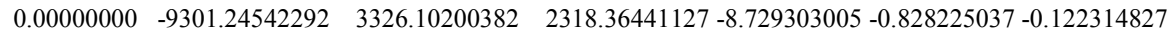
$120.00000000-44672.91239679-6213.11996581-1738.80131727-3.719475070-1.336673022-0.621888261$ $240.00000000-67053.08885387-14994.69685946-5897.99072793-2.860576613-1.183771565-0.568473909$ $360.00000000-85227.84253168-22897.08484471-9722.59184564-2.426469823-1.078592475-0.525341431$ $480.00000000-100986.00419136-30171.19698695-13283.77044765-2.147108978-1.000530827-0.491587582$ $600.00000000-115093.00686386-36962.56316477-16634.15682929-1.945446188-0.938947736-0.464199202$ $720.00000000-127965.80064891-43363.32967165-19809.90480432-1.789652016-0.888278463-0.441254468$ $840.00000000-139863.28332206-49436.45704153-22836.80438139-1.663762568-0.845315913-0.421548627$ $960.00000000-150960.22978258-55227.45413896-25734.01408879-1.558730986-0.808061065-0.404293846$ $1080.00000000-161381.71414630-60770.64040903-28516.26290017-1.468977174-0.775190459-0.388951810$ $1200.00000000-171221.18736947-66092.76474442-31195.19847387-1.390837596-0.745785633-0.375140398$ $1320.00000000-180550.82888745-71215.23290630-33780.24938270-1.321788672-0.719184752-0.362579495$ $1440.00000000-189427.87533074-76155.54943344-36279.19882816-1.260024473-0.694896053-0.351058133$ $1560.00000000-197898.69401408-80928.29015181-38698.57972447-1.204211888-0.672544709-0.340413731$ $1600.00000000-200638.82986236-82484.14969882-39488.34331447-1.186748462-0.665472422-0.337037582$

$23599 \mathrm{xx}$

$\begin{array}{llllllll}0.00000000 & 9892.63794341 & 35.76144969 & -1.08228838 & 3.556643237 & 6.456009375 & 0.783610890\end{array}$ $\begin{array}{lllllll}20.00000000 & 11931.95642997 & 7340.74973750 & 886.46365987 & 0.308329116 & 5.532328972 & 0.672887281\end{array}$ $\begin{array}{lllllll}40.00000000 & 11321.71039205 & 13222.84749156 & 1602.40119049 & -1.151973982 & 4.285810871 & 0.521919425\end{array}$ $\begin{array}{lllllll}60.00000000 & 9438.29395675 & 17688.05450261 & 2146.59293402 & -1.907904054 & 3.179955046 & 0.387692479\end{array}$ $\begin{array}{llllllll}80.00000000 & 6872.08634639 & 20910.11016811 & 2539.79945034 & -2.323995367 & 2.207398462 & 0.269506121\end{array}$ $100.00000000 \quad 3933.37509798 \quad 23024.07662542 \quad 2798.25966746-2.542860616 \quad 1.327134966 \quad 0.162450076$ $\begin{array}{lllllll}120.00000000 & 816.64091546 & 24118.98675475 & 2932.69459428 & -2.626838010 & 0.504502763 & 0.062344306\end{array}$ $140.00000000-2334.41705804 \quad 24246.86096326 \quad 2949.36448841-2.602259646-0.288058266-0.034145135$

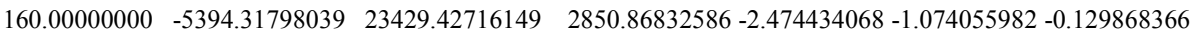

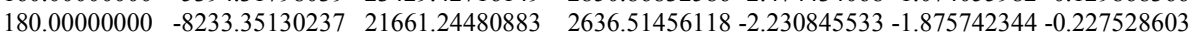
$200.00000000-10693.96497348 \quad 18909.88168891 \quad 2302.33707548-1.835912433-2.716169865-0.329931880$ $220.00000000-12553.89669904 \quad 15114.63990716 \quad 1840.93573231-1.212478879-3.619036996-0.439970633$ $240.00000000-13450.20591864 \quad 10190.57904289 \quad 1241.95958736-0.189082511-4.596701971-0.559173899$ $260.00000000-12686.60437121 \quad 4079.31106161 \quad 498.27078614 \quad 1.664498211-5.559889865-0.676747779$ $\begin{array}{lllllll}280.00000000 & -8672.55867753 & -2827.56823315 & -342.59644716 & 5.515079852 & -5.551222962 & -0.676360044\end{array}$ $\begin{array}{llllllll}300.00000000 & 1153.31498060 & -6411.98692060 & -779.87288941 & 9.689818102 & 1.388598425 & 0.167868798\end{array}$ $\begin{array}{llllllll}320.00000000 & 9542.79201056 & -533.71253081 & -65.73165428 & 3.926947087 & 6.459583539 & 0.785686755\end{array}$ $\begin{array}{llllllll}340.00000000 & 11868.80960100 & 6861.59590848 & 833.72780602 & 0.452957852 & 5.632811328 & 0.685262323\end{array}$ $\begin{array}{llllllll}360.00000000 & 11376.23941678 & 12858.97121366 & 1563.40660172 & -1.087665695 & 4.374693347 & 0.532207051\end{array}$ $\begin{array}{lllllll}380.00000000 & 9547.70300782 & 17421.48570758 & 2118.56907515 & -1.876540262 & 3.253891728 & 0.395810243\end{array}$ $\begin{array}{lllllll}400.00000000 & 7008.51470263 & 20725.47471227 & 2520.56064289 & -2.308703599 & 2.270724438 & 0.276138613\end{array}$ $\begin{array}{llllllll}420.00000000 & 4082.28135104 & 22911.04184601 & 2786.37568309 & -2.536665546 & 1.383670232 & 0.168153407\end{array}$ $\begin{array}{lllllll}440.00000000 & 969.17978149 & 24071.23673676 & 2927.31326579 & -2.626695115 & 0.557172428 & 0.067536854\end{array}$

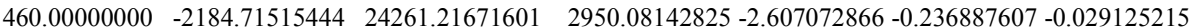
$\begin{array}{lllll}480.00000000 & -5253.42223370 & 23505.37595671 & 2857.66120738-2.484424544-1.022255436 & -0.124714444\end{array}$ $500.00000000 \quad-8108.27961017 \quad 21800.81688388 \quad 2649.72981961-2.247597251-1.821159176-0.221925624$ $520.00000000-10594.77795556 \quad 19117.80779221 \quad 2322.72136979-1.863118484-2.656426668-0.323521502$ $540.00000000-12497.32045995 \quad 15398.64085906 \quad 1869.69983897-1.258130763-3.551583368-0.432338888$

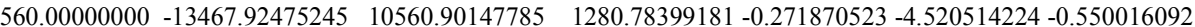

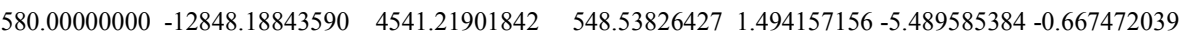

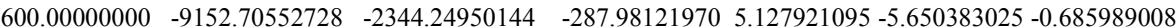
$\begin{array}{lllllll}620.00000000 & 280.38490909 & -6500.10264018 & -790.36092984 & 9.779619614 & 0.581815811 & 0.074171345\end{array}$ $\begin{array}{llllllll}640.00000000 & 9166.25784315 & -1093.12552651 & -129.49428887 & 4.316668714 & 6.438636494 & 0.785116609\end{array}$ $\begin{array}{lllllll}660.00000000 & 11794.48942915 & 6382.21138354 & 780.88439015 & 0.604412453 & 5.731729369 & 0.697574333\end{array}$ $\begin{array}{lllllll}680.00000000 & 11424.30138324 & 12494.26088864 & 1524.33165488 & -1.021328075 & 4.463448968 & 0.542532698\end{array}$ $\begin{array}{lllllll}700.00000000 & 9652.09867350 & 17153.84762075 & 2090.48038336 & -1.844516637 & 3.327522235 & 0.403915232\end{array}$

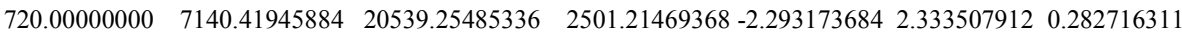

$24208 \times x$

$0.00000000 \quad 7534.10987189 \quad 41266.39266843$

$-0.10801028-3.027168008 \quad 0.558848996 \quad 0.207982755$ $120.00000000-14289.19940414 \quad 39469.05530051 \quad 1428.62838591-2.893205245-1.045447840 \quad 0.179634249$ $\begin{array}{llllll}240.00000000 & -32222.92014955 & 26916.25425799 & 2468.59996594-1.973007929 & -2.359335071 & 0.102539376\end{array}$ 


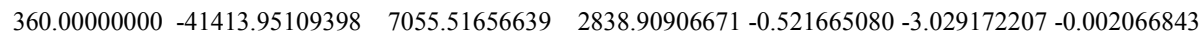
$480.00000000 \quad-39402.72251896-14716.42475223 \quad 2441.32678358 \quad 1.066928187-2.878714619-0.105865729$ $600.00000000-26751.08889828-32515.13982431 \quad 1384.388655702 .366228869-1.951032799-0.181018498$ $720.00000000 \quad-6874.77975542-41530.38329422 \quad-46.602454593 .027415087-0.494671177-0.207337260$ $\begin{array}{lllllll}840.00000000 & 14859.52039042 & -39302.58907247 & -1465.02482524 & 2.869609883 & 1.100123969 & -0.177514425\end{array}$ $\begin{array}{llllllll}960.00000000 & 32553.14863770 & -26398.88401807 & -2485.45866002 & 1.930064459 & 2.401574539 & -0.099250520\end{array}$

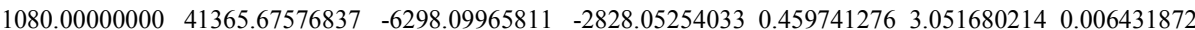
$\begin{array}{llllllll}1200.00000000 & 38858.83295070 & 15523.39314924 & -2396.86850752 & -1.140211488 & 2.867567143 & 0.110637217\end{array}$ $1320.00000000 \quad 25701.46068162 \quad 33089.42617648-1308.68556638-2.4287138211 .8973814310 .184605907$ $\begin{array}{lllllll}1440.00000000 & 5501.08137100 & 41590.27784405 & 138.32522930 & -3.050691874 & 0.409203052 & 0.207958133\end{array}$

$25954 \mathrm{xx}$

$0.00000000 \quad 8827.15660472-41223.00971237$ $-1440.00000000 \quad 8118.18519221-41368.40537378$ $\begin{array}{lll}-1320.00000000 & 27766.34015328 & -31724.97000557\end{array}$ $-1200.00000000 \quad 39932.57237973-13532.60040454$ $\begin{array}{lll}-1080.00000000 & 41341.01365441 & 8305.71681955\end{array}$ $-960.00000000 \quad 31614.99210558 \quad 27907.29155353$ $\begin{array}{llll}-840.00000000 & 13375.75227587 & 39994.27017651\end{array}$ $\begin{array}{llll}-720.00000000 & -8464.89963309 & 41312.93549892\end{array}$ $\begin{array}{llll}-600.00000000 & -28026.23406158 & 31507.89995661\end{array}$ $\begin{array}{llll}-480.00000000 & -40040.01314363 & 13218.00579413\end{array}$ $\begin{array}{llll}-360.00000000 & -41268.43291976 & -8632.06859693\end{array}$ $-240.00000000-31377.85317015-28156.13970334$ $\begin{array}{llll}-120.00000000 & -13031.41552688 & -40092.33381029\end{array}$ $\begin{array}{llll}0.00000000 & 8827.15660472 & -41223.00971237\end{array}$ $120.00000000 \quad 28306.85426674 \quad-31243.80147394$ $240.00000000 \quad 40159.05128805-12845.39151157$ $360.00000000 \quad 41192.55903455 \quad 9013.79606759$ $480.00000000 \quad 31131.69755798 \quad 28445.55681731$ $600.00000000 \quad 12687.81846530 \quad 40217.83324639$ $\begin{array}{llll}720.00000000 & -9172.23500245 & 41161.63475527\end{array}$ $\begin{array}{llll}840.00000000 & -28562.51093192 & 31022.45987587\end{array}$ $960.00000000-40260.77504549 \quad 12529.11484344$ $1080.00000000 \quad-41114.14376538-9338.87194483$ $1200.00000000-30890.01512240-28690.40750792$ $1320.00000000-12341.46194020-40310.06316386$ $1440.00000000 \quad 9533.27750818-41065.52390214$

$26900 \mathrm{xx}$

$\begin{array}{lll}0.00000000 & -42014.83795787 \quad 3702.34357772\end{array}$ $\begin{array}{cccc}9300.00000000 & 40968.68133298 & -9905.99156086\end{array}$ $\begin{array}{llll}9360.00000000 & 42135.66858481 & 1072.99195618\end{array}$ $\begin{array}{llll}9400.00000000 & 41304.75156132 & 8398.27742944\end{array}$ $\begin{array}{lllll}3.63482963 & 3.007087319 & 0.643701323 & 0.000941663\end{array}$ $\begin{array}{lllll}4.11046687 & 3.017696741 & 0.591994297 & 0.000933016\end{array}$ $\begin{array}{lllll}9.93297846 & 2.314236153 & 2.024903193 & 0.000660861\end{array}$ $\begin{array}{llllll}13.12958252 & 0.987382819 & 2.911942843 & 0.000213298\end{array}$ $12.84988501-0.6050982243 .014378268-0.000291034$ $9.16618797-2.0342435232 .305014102-0.000718418$ $3.05416854-2.9154243660 .975119874-0.000955576$ $-3.86622919-3.011600615-0.617275050-0.000939664$ $-9.76047869-2.296840160-2.043607595-0.000674889$ $\begin{array}{llll}-13.06594832 & -0.963328772 & -2.919827983 & -0.000231414\end{array}$ $\begin{array}{llllll}-12.90661266 & 0.630042315 & -3.009677376 & 0.000273163\end{array}$ $\begin{array}{llll}-9.32605530 & 2.054021717 & -2.288554158 & 0.000704959\end{array}$ $\begin{array}{lllll}-3.27636660 & 2.924657466 & -0.950541167 & 0.000949381\end{array}$ $\begin{array}{llllll}3.63482963 & 3.007087319 & 0.643701323 & 0.000941663\end{array}$ $\begin{array}{llllll}9.57216891 & 2.279137743 & 2.064316875 & 0.000684127\end{array}$ $\begin{array}{lllll}12.96086316 & 0.937265422 & 2.928448287 & 0.000245505\end{array}$ $12.90495666-0.6567274423 .003543458-0.000257479$ $9.42419238-2.0734848422 .269770851-0.000691233$ $3.44726249-2.931721827 \quad 0.924962230-0.000940766$ $-3.43575757-3.000571486-0.668847508-0.000940101$ $-9.39562161-2.261449202-2.082713897-0.000689669$ $-12.84915105-0.913097031-2.935933528-0.000256181$ $\begin{array}{llll}-12.87952404 & 0.681588815 & -2.998432565 & 0.000245006\end{array}$ $\begin{array}{llll}-9.48037212 & 2.092989805 & -2.252978152 & 0.000680459\end{array}$ $\begin{array}{lllll}-3.55833201 & 2.940537098 & -0.900219523 & 0.000934170\end{array}$ $\begin{array}{llll}3.30756482 & 2.995596171 & 0.695200236 & 0.000938525\end{array}$

$26975 \mathrm{xx}$

$\begin{array}{lllllll}0.00000000 & -14506.92313768 & -21613.56043281 & 10.05018894 & 2.212943308 & 1.159970892 & 3.020600202\end{array}$ $\begin{array}{lllllll}120.00000000 & 7309.62197950 & 6076.00713664 & 6800.08705263 & 1.300543383 & 5.322579615 & -4.788746312\end{array}$ $240.00000000-3882.62933791 \quad 11960.00543452-25088.14383845-2.146773699-1.372461491-2.579382089$ $360.00000000-16785.45507465 \quad-734.79159704-34300.57085853-1.386528125-1.907762641-0.220949641$ $480.00000000-23524.16689356-13629.45124622-30246.27899200-0.462846784-1.586139830 \quad 1.269293624$ $600.00000000-22890.23597092-22209.35900155-16769.91946116 \quad 0.704351342-0.6711125942 .432433851$

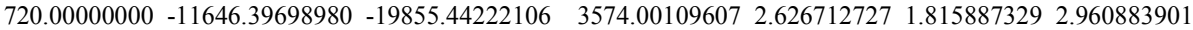
$840.00000000 \quad 7665.76124241 \quad 11159.78946577 \quad 345.93813117-0.584818007 \quad 3.193514161-5.750338922$ $960.00000000-6369.35388112 \quad 10204.80073022-27844.52150384-2.050573276-1.582940542-2.076075232$ $1080.00000000-18345.64763145 \quad-2977.76684430-34394.90760612-1.243589864-1.892050757 \quad 0.060372061$ $1200.00000000-23979.74839255-15436.44139571-28616.50540218-0.294973425-1.482987916 \quad 1.478255628$ $1320.00000000-21921.97167880-22852.45147658 \quad-13784.85308485 \quad 0.945455629-0.4289409952 .596964378$ $1440.00000000 \quad-8266.43821031-17210.74590112 \quad 6967.955460703 .0822440692 .665881872 \quad 2.712555075$ 
$\begin{array}{lllllll}1560.00000000 & 6286.85464535 & 13809.56328971 & -6321.60663781 & -1.615964016 & 1.383135377 & -5.358719132\end{array}$ $1680.00000000 \quad-8730.87526788 \quad 8244.63344365-30039.92372791-1.935622871-1.724162072-1.631224738$ $1800.00000000-19735.81883249-5191.76593007-34166.14974143-1.097835530-1.860148418 \quad 0.324401050$ $1920.00000000-24232.73847703-17112.08243255-26742.88893252-0.119786184-1.364365317 \quad 1.680220468$ $2040.00000000-20654.45640708-23184.54386047-10611.55144716 \quad 1.209238113-0.1441696392 .748054938$

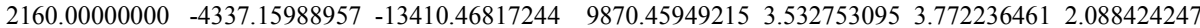
$\begin{array}{lllllll}2280.00000000 & 4074.62263523 & 14698.07548285 & -12248.65327973 & -2.053824693 & 0.203325817 & -4.607867718\end{array}$ $2400.00000000-10950.23438984 \quad 6148.66879447-31736.65532865-1.809875605-1.816179062-1.233364913$ $2520.00000000-20952.40702045-7358.71507895-33633.06643074-0.948973031-1.8135941370 .573893078$ $2640.00000000-24273.48944134-18637.15546906-24633.27702390 \quad 0.064161440-1.228537560 \quad 1.875728935$ $\begin{array}{llllllll}2760.00000000 & -19057.55468077 & -23148.29322082 & -7269.38614178 & 1.500802809 & 0.195383037 & 2.879031237\end{array}$ $\begin{array}{llllllll}2880.00000000 & 43.69305308 & -8145.90299207 & 11634.57079913 & 3.780661682 & 5.105315423 & 0.714401345\end{array}$

$28057 \mathrm{xx}$

$\begin{array}{lllllll}0.00000000 & -2715.28237486 & -6619.26436889 & -0.01341443 & -1.008587273 & 0.422782003 & 7.385272942\end{array}$

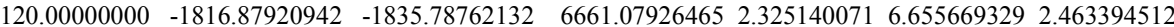
$240.00000000 \quad 1483.17364291 \quad 5395.21248786 \quad 4448.65907172 \quad 2.5605403874 .039025766-5.736648561$ $360.00000000 \quad 2801.25607157 \quad 5455.03931333 \quad-3692.12865694-0.595095864-3.951923117-6.298799125$ $\begin{array}{lllllll}480.00000000 & 411.09332812 & -1728.99769152 & -6935.45548810 & -2.935970964 & -6.684085058 & 1.492800886\end{array}$ $\begin{array}{llllllll}600.00000000 & -2506.52558454 & -6628.98655094 & -988.07784497 & -1.390577189 & -0.556164143 & 7.312736468\end{array}$

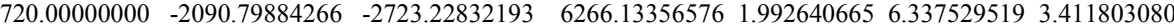
$840.00000000 \quad 1091.80560222 \quad 4809.88229503 \quad 5172.428978942 .717483546 \quad 4.805518977-5.030019896$ $960.00000000 \quad 2811.14062300 \quad 5950.65707171 \quad-2813.23705389-0.159662742-3.121215491-6.775341949$ $\begin{array}{lllllll}1080.00000000 & 805.72698304 & -812.16627907 & -7067.58483968 & -2.798936020 & -6.889265977 & 0.472770873\end{array}$ $1200.00000000 \quad-2249.59837532 \quad-6505.84890714-1956.72365062-1.731234729-1.5287502307 .096660885$

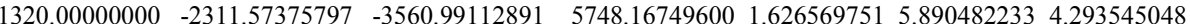
$\begin{array}{lllllll}1440.00000000 & 688.16056594 & 4124.87618964 & 5794.55994449 & 2.810973665 & 5.479585563 & -4.224866316\end{array}$ $\begin{array}{llllllll}1560.00000000 & 2759.94088230 & 6329.87271798 & -1879.19518331 & 0.266930672 & -2.222670878 & -7.119390567\end{array}$

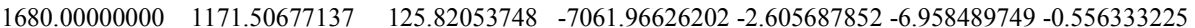
$1800.00000000 \quad-1951.43708472 \quad-6251.71945820 \quad-2886.95472355-2.024131483-2.475214272 \quad 6.741537478$

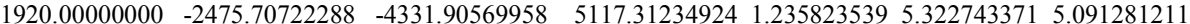
$\begin{array}{llllllll}2040.00000000 & 281.46097847 & 3353.51057102 & 6302.87900650 & 2.840647273 & 6.047222485 & -3.337085992\end{array}$ $\begin{array}{lllllll}2160.00000000 & 2650.33118860 & 6584.33434851 & -908.29027134 & 0.675457235 & -1.274044972 & -7.323921567\end{array}$ $2280.00000000 \quad 1501.17226597 \quad 1066.31132756-6918.71472952-2.361891904-6.889669974-1.574718619$ $2400.00000000 \quad-1619.73468334-5871.14051991 \quad-3760.56587071-2.264093975-3.376316601 \quad 6.254622256$ $\begin{array}{lllllll}2520.00000000 & -2581.04202505 & -5020.05572531 & 4385.92329047 & 0.829668458 & 4.645048038 & 5.789262667\end{array}$ $\begin{array}{lllllll}2640.00000000 & -119.22080628 & 2510.90620488 & 6687.45615459 & 2.807575712 & 6.496549689 & -2.384136661\end{array}$

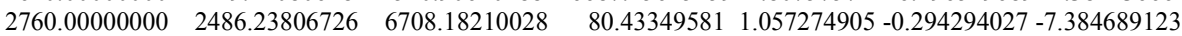
$2880.00000000 \quad 1788.42334580 \quad 1990.50530957 \quad-6640.59337725-2.074169091-6.683381288-2.562777776$

$28129 \mathrm{xx}$

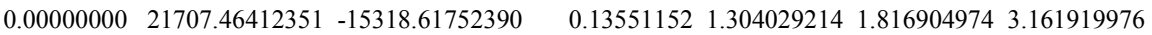
$120.00000000 \quad 18616.75971861 \quad 3166.15177043 \quad 18833.41523210-2.0761220162 .838457575 \quad 1.586210535$ $240.00000000 \quad-3006.50596328 \quad 18522.20742011 \quad 18941.84078154-3.3754527891 .032680773-1.559324534$ $360.00000000-21607.02086957 \quad 15432.59962630 \quad 206.62470309-1.306049851-1.817011568-3.163725018$ $480.00000000-18453.06134549-3150.83256134-18685.830309362 .106017925-2.860236337-1.586151870$ $600.00000000 \quad 3425.11742384-18514.73232706-18588.67200557 \quad 3.394666340-1.003072030 \quad 1.610061295$ $\begin{array}{llllllll}720.00000000 & 21858.23838149 & -15101.51661554 & 387.34517048 & 1.247973967 & 1.856017403 & 3.161439948\end{array}$ $840.00000000 \quad 18360.69935796 \quad 3506.55256762 \quad 19024.81678979-2.1226841842 .830618605 \quad 1.537510677$ $\begin{array}{lllllll}960.00000000 & -3412.84765409 & 18646.85269710 & 18748.00359987 & -3.366815728 & 0.986039922 & -1.607874972\end{array}$ $1080.00000000-21758.08331586 \quad 15215.44829478 \quad-180.82181406-1.250144680-1.856490448-3.163774870$ $1200.00000000-18193.41290284 \quad-3493.85876912-18877.14757717 \quad 2.153326942-2.852221264-1.536617760$ $\begin{array}{lllllllll}1320.00000000 & 3833.57386848 & -18635.77026711 & -18388.68722886 & 3.384748179 & -0.955363841 & 1.658785020\end{array}$ $1440.00000000 \quad 22002.20074562 \quad-14879.72595593 \quad 774.328270991 .1915736191 .8945611653 .159953047$

$28350 \mathrm{xx}$

$\begin{array}{lllllll}0.00000000 & 6333.08123128 & -1580.82852326 & 90.69355720 & 0.714634423 & 3.224246550 & 7.083128132\end{array}$ $120.00000000 \quad-3990.93845855 \quad 3052.98341907 \quad 4155.32700629-5.909006188-0.876307966-5.039131404$ $\begin{array}{lllllll}240.00000000 & -603.55232010 & -2685.13474569 & -5891.70274282 & 7.572519907 & -1.975656726 & 0.121722605\end{array}$ 
$\begin{array}{lllllll}360.00000000 & 4788.22345627 & 782.56169214 & 4335.14284621 & -4.954509026 & 3.683346464 & 4.804645839\end{array}$ $480.00000000 \quad-6291.84601644 \quad 1547.82790772 \quad-453.67116498-0.308625588-3.341538574-7.082659115$

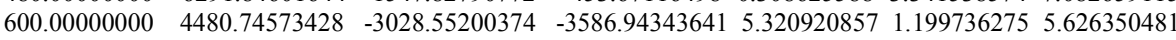

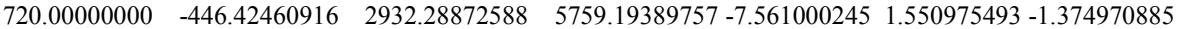
$840.00000000 \quad-3713.79581831 \quad-1382.66125130 \quad-5122.45131136 \quad 6.090931626-3.512629733-3.467571746$ $\begin{array}{lllllll}960.00000000 & 6058.32017522 & -827.47406722 & 2104.04678651 & -1.798403024 & 3.787067272 & 6.641439744\end{array}$ $1080.00000000 \quad-5631.73659006 \quad 2623.70953644 \quad 1766.49125084-3.216401578-2.309140959-6.788609120$ $\begin{array}{lllllll}1200.00000000 & 2776.84991560 & -3255.36941953 & -4837.19667790 & 6.748135564 & -0.193044825 & 4.005718698\end{array}$ $\begin{array}{lllllll}1320.00000000 & 1148.04430837 & 2486.07343386 & 5826.34075913 & -7.420162295 & 2.589456382 & 0.356350006\end{array}$

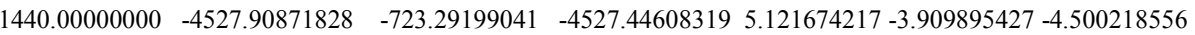

$28623 \mathrm{xx}$

$\begin{array}{lllllll}0.00000000 & -11665.70902324 & 24943.61433357 & 25.80543633 & -1.596228621 & -1.476127961 & 1.126059754\end{array}$ $120.00000000 \quad-11645.35454950 \quad 979.37668356 \quad 5517.89500058 \quad 3.407743502-5.183094988-0.492983277$ $\begin{array}{llllllll}240.00000000 & 5619.19252274 & 19651.44862280 & -7261.38496765 & -2.013634213 & 3.106842861 & 0.284235517\end{array}$ $360.00000000 \quad-9708.68629714 \quad 26306.14553149 \quad-1204.29478856-1.824164290-0.9319095961 .113419052$ $\begin{array}{llllllll}480.00000000 & -14394.03162892 & 6659.30765074 & 5593.38345858 & 1.556522911 & -4.681657614 & 0.296912248\end{array}$ $600.00000000 \quad 7712.09476270 \quad 15565.72627434 \quad-7342.40465571-1.6468003644 .070313571-0.109483081$ $\begin{array}{llllllll}720.00000000 & -7558.36739603 & 27035.11367962 & -2385.12054184 & -1.999583791 & -0.393409283 & 1.078093515\end{array}$ $\begin{array}{llllllll}840.00000000 & -15495.61862220 & 11550.15897828 & 5053.83178121 & 0.469277336 & -4.029761073 & 0.679054742\end{array}$ $\begin{array}{lllllll}960.00000000 & 9167.02568222 & 10363.65204210 & -6871.52576042 & -0.881621027 & 5.223361510 & -0.740696297\end{array}$ $\begin{array}{llllllll}1080.00000000 & -5275.80272094 & 27151.78486008 & -3494.50687216 & -2.129609388 & 0.150196480 & 1.021038089\end{array}$ $1200.00000000-15601.37656145 \quad 15641.29379850 \quad 4217.03266850-0.249183123-3.405238557 \quad 0.888214503$ $\begin{array}{llllllll}1320.00000000 & 9301.05872300 & 3883.15265574 & -5477.86477017 & 0.871447821 & 6.493677331 & -1.885545282\end{array}$ $\begin{array}{lllllll}1440.00000000 & -2914.31065828 & 26665.20392758 & -4511.09814335 & -2.216261909 & 0.710067769 & 0.940691824\end{array}$

$28626 \mathrm{xx}$

$0.00000000 \quad 42080.71852213 \quad-2646.86387436$ $\begin{array}{ccc}120.00000000 & 37740.00085593 & 18802.76872802\end{array}$ $240.00000000 \quad 23232.82515008 \quad 35187.33981802$ $\begin{array}{llll}360.00000000 & 2467.44290178 & 42093.60909959\end{array}$ $\begin{array}{llll}480.00000000 & -18962.59052991 & 37661.66243819\end{array}$ $600.00000000 \quad-35285.00095313 \quad 23085.44402778$ $\begin{array}{llll}720.00000000 & -42103.20138132 & 2291.06228893\end{array}$ $840.00000000-37580.31858370-19120.40485693$ $960.00000000-22934.20761876-35381.23870806$ $1080.00000000 \quad-2109.90332389-42110.71508198$ $1200.00000000 \quad 19282.77774728-37495.59250598$ $1320.00000000 \quad 35480.60990600-22779.03375285$ $1440.00000000 \quad 42119.96263499 \quad-1925.77567263$

$28872 \mathrm{xx}$

$\begin{array}{llll}0.00000000 & -6131.82730456 & 2446.52815528\end{array}$ $\begin{array}{lll}5.00000000 & -5799.24256134 & 2589.14811119\end{array}$

$\begin{array}{lll}10.00000000 & -4769.05061967 & 2420.46580562\end{array}$

$\begin{array}{lll}15.00000000 & -3175.45157340 & 1965.98738086\end{array}$

$20.00000000 \quad-1210.19024802 \quad 1281.54541294$

$\begin{array}{lll}25.00000000 & 896.73799533 & 447.12357305\end{array}$

$30.00000000 \quad 2896.99663534 \quad-440.04738594$

$\begin{array}{llll}35.00000000 & 4545.78970167 & -1273.55952872\end{array}$

$40.00000000 \quad 5627.43299371 \quad-1947.94282469$

$45.00000000 \quad 5984.72318534-2371.37691609$

$50.00000000 \quad 5548.43325922 \quad-2480.16469245$

$29141 \mathrm{xx}$

0.00000000 20.00000000

$423.99295524-6658.12256149$

20.00000000
40.00000000

$31.80883587-1017.17852239$

$-83.44906141 \quad 6286.20208453$ $\begin{array}{llllll}0.81851294 & 0.193105177 & 3.068688251 & 0.000438449\end{array}$ $\begin{array}{llll}3.45512584 & -1.371035206 & 2.752105932 & 0.000336883\end{array}$ $\begin{array}{lllll}4.98927428 & -2.565776620 & 1.694193132 & 0.000163365\end{array}$ $\begin{array}{lllll}5.15062987 & -3.069341800 & 0.179976276 & -0.000031739\end{array}$ $4.04433258-2.746151982-1.382675777-0.000197633$ $2.08711880-1.683277908-2.572893625-0.000296282$ $-0.13274964-0.166974816-3.070104560-0.000311007$ $-2.02755702 \quad 1.394367848-2.740341612-0.000248591$ $-3.164959322 .580167539-1.672360951-0.000134907$ $\begin{array}{llll}-3.36507889 & 3.070935369 & -0.153808390 & -0.000005855\end{array}$ $\begin{array}{lllll}-2.71861462 & 2.734400524 & 1.406220933 & 0.000103486\end{array}$ $\begin{array}{lllll}-1.52841859 & 1.661210676 & 2.587414593 & 0.000168300\end{array}$ $\begin{array}{lllll}-0.19827433 & 0.140521206 & 3.071541613 & 0.000179561\end{array}$

$\begin{array}{lllll}-253.64211033 & -0.144920228 & 0.995100963 & 7.658645067\end{array}$ $\begin{array}{lllll}2011.54515100 & 2.325207364 & -0.047125672 & 7.296234071\end{array}$ $4035.30855837 \quad 4.464585796-1.0609232096 .070907874$ $\begin{array}{lllll}5582.12569607 & 6.049639376 & -1.935777558 & 4.148607019\end{array}$ $6474.68172772 \quad 6.920746273-2.580517337 \quad 1.748783868$ $6607.22400507 \quad 6.983396282-2.925846168-0.872655207$ $5954.92675486 \quad 6.211488246-2.926949815-3.433959806$ $4580.165129844 .656984233-2.568711513-5.638510954$ $2634.167149302 .464141047-1.873985161-7.195743032$ $349.87996209-0.121276950-0.911981546-7.859613894$ $-1979.24314527-2.7632695340 .199691915-7.482796996$ $2223.49837161-1.1135159742 .530970283-7.219445568$ 


\begin{abstract}
$60.00000000 \quad-958.57681221 \quad 3259.26005348 \quad-5722.63732467-0.101225813-6.735338321-3.804851872$

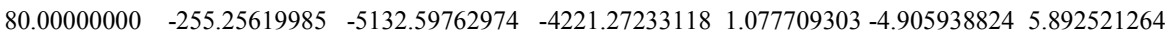
$\begin{array}{llllllll}100.00000000 & 867.44295097 & -5038.40402933 & 4256.73810533 & 0.479447535 & 5.032326446 & 5.857126248\end{array}$ $\begin{array}{lllllll}120.00000000 & 559.16882013 & 3376.30587937 & 5699.22017391 & -0.906749328 & 6.646149867 & -3.852331832\end{array}$ $140.00000000 \quad-669.85184205 \quad 6196.00229484-2281.95741770-0.795804092-2.752114827-7.202478520$ $\begin{array}{lllllll}160.00000000 & -784.20708019 & -1278.53125553 & -6449.19892596 & 0.636702380 & -7.595425203 & 1.431090802\end{array}$ $\begin{array}{llllllll}180.00000000 & 406.15811659 & -6607.03115799 & 148.33021477 & 1.009818575 & 0.231843765 & 7.692047844\end{array}$ $\begin{array}{llllllll}200.00000000 & 916.34911813 & -884.08649248 & 6491.09810362 & -0.302163049 & 7.669887109 & 1.084336909\end{array}$ $\begin{array}{lllllll}220.00000000 & -104.02490970 & 6304.31821405 & 1960.08739882 & -1.108873823 & 2.259522809 & -7.351147710\end{array}$ $240.00000000 \quad-944.61642849 \quad 2872.17248379 \quad-5846.94103362-0.051117686-6.989747076-3.413102600$ $\begin{array}{lllllll}260.00000000 & -187.16569888 & -5404.86163467 & -3731.97057618 & 1.094696706 & -4.412110995 & 6.326060952\end{array}$ $\begin{array}{llllllll}280.00000000 & 884.59720467 & -4465.74516163 & 4725.83632696 & 0.380656028 & 5.691554046 & 5.303910983\end{array}$ $\begin{array}{lllllll}300.00000000 & 446.40767236 & 4086.66839620 & 5093.05596650 & -0.982424447 & 6.072965199 & -4.791630682\end{array}$ $320.00000000 \quad-752.24467495 \quad 5588.35473301 \quad-3275.04092573-0.661161370-4.016290740-6.676898026$ $\begin{array}{lllllll}340.00000000 & -643.72872525 & -2585.02528560 & -5923.01306608 & 0.807922142 & -7.171597814 & 3.041115058\end{array}$ $\begin{array}{lllllll}360.00000000 & 584.40295819 & -6202.35605817 & 1781.00536019 & 0.869250450 & 2.226927514 & 7.471676765\end{array}$ $\begin{array}{lllllll}380.00000000 & 779.59211765 & 1100.73728301 & 6311.59529480 & -0.599552305 & 7.721032522 & -1.275153027\end{array}$ $400.00000000 \quad-403.03155588 \quad 6399.18000837 \quad-364.12735875-1.008861924-0.516636615-7.799812287$ $\begin{array}{lllllll}420.00000000 & -852.93910071 & 192.65232023 & -6322.47054784 & 0.396006194 & -7.882964919 & -0.289331517\end{array}$
\end{abstract}

$29238 \times x$

$\begin{array}{lllllll}0.00000000 & -5566.59512819 & -3789.75991159 & 67.60382245 & 2.873759367 & -3.825340523 & 6.023253926\end{array}$ $120.00000000 \quad 4474.27915495 \quad-1447.72286142 \quad 4619.83927235 \quad 4.712595822 \quad 5.668306153-2.701606741$ $240.00000000 \quad 1922.17712474 \quad 5113.01138342-4087.08470203-6.490769651-0.522350158-3.896001154$

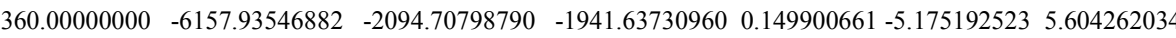
$\begin{array}{lllllll}480.00000000 & 2482.64052411 & -3268.45944555 & 5146.38006190 & 6.501814698 & 4.402848754 & -0.350943511\end{array}$ $600.00000000 \quad 4036.26455287 \quad 4827.43347201 \quad-2507.99063955-5.184409515 \quad 1.772280695-5.331390168$ $720.00000000 \quad-5776.81371622 \quad-118.64155319 \quad-3641.22052418-2.539917207-5.622701582 \quad 4.403125405$ $\begin{array}{llllllll}840.00000000 & 67.98699487 & -4456.49213473 & 4863.71794283 & 7.183809420 & 2.418917791 & 2.015642495\end{array}$ $\begin{array}{lllllll}960.00000000 & 5520.62207038 & 3782.38203554 & -596.73193161 & -3.027966069 & 3.754152525 & -6.013506363\end{array}$

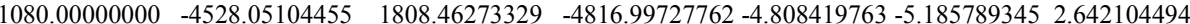

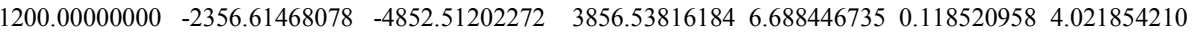
$\begin{array}{llllllll}1320.00000000 & 6149.65800134 & 2173.59423261 & 1369.29488732 & -0.345832777 & 5.109857861 & -5.842951828\end{array}$ $1440.00000000 \quad-2629.55011449 \quad 3400.98040158 \quad-5344.38217129-6.368548448-3.998963509 \quad 0.577253064$

$88888 \mathrm{xx}$

$\begin{array}{lllllll}0.00000000 & 2328.96975262 & -5995.22051338 & 1719.97297192 & 2.912073281 & -0.983417956 & -7.090816210\end{array}$ $\begin{array}{llllllll}120.00000000 & 1020.69234558 & 2286.56260634 & -6191.55565927 & -3.746543902 & 6.467532721 & 1.827985678\end{array}$ $\begin{array}{lllllll}240.00000000 & -3226.54349155 & 3503.70977525 & 4532.80979343 & 1.000992116 & -5.788042888 & 5.162585826\end{array}$ $\begin{array}{lllllll}360.00000000 & 2456.10706533 & -6071.93855503 & 1222.89768554 & 2.679390040 & -0.448290811 & -7.228792155\end{array}$ $\begin{array}{lllllll}480.00000000 & 787.16457349 & 2719.91800946 & -6043.86662024 & -3.759883839 & 6.277439314 & 2.397897864\end{array}$ $600.00000000 \quad-3110.97648029 \quad 3121.73026235 \quad 4878.15217035 \quad 1.244916056-6.124880425 \quad 4.700576353$ $\begin{array}{llllllll}720.00000000 & 2567.56229695 & -6112.50383922 & 713.96374435 & 2.440245751 & 0.098109002 & -7.319959258\end{array}$ $840.00000000 \quad 556.05661780 \quad 3144.52288201 \quad-5855.34636178-3.7546601436 .044752775 \quad 2.957941672$ $\begin{array}{llllllll}960.00000000 & -2982.47940539 & 2712.61663711 & 5192.32330472 & 1.475566773 & -6.427737014 & 4.202420227\end{array}$

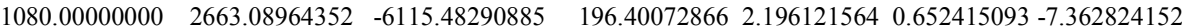
$1200.00000000 \quad 328.54999674 \quad 3557.09490552 \quad-5626.21427211-3.7311932885 .769341172 \quad 3.504058731$

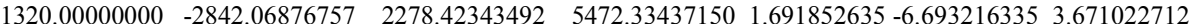
$\begin{array}{lllllll}1440.00000000 & 2742.55398832 & -6079.67009123 & -326.39012649 & 1.948497651 & 1.211072678 & -7.356193131\end{array}$ 


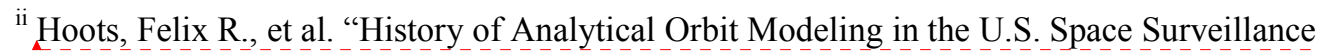

Deleted: 9

System.” Journal of Guidance Control, and Dynamics March-April 2004: 174-185.

iii Vallado, David, et al. 'Revisiting Spacetrack Report \#3', AIAA 2006-6753,

Formatted: Font: 12 pt

iv Vallado, David, et al. 'Revisiting Spacetrack Report \#3', AIAA 2006-6753

Formatted: Line spacing:

Double

` Abercromby, Kira. “Object 8832” E-Mail to N. Miura, 26 May 2009.

vi "Selected Orbital Data" 2009. <http://www.heavens-above.com>

Formatted: Normal, Line

vii http://en.wikipedia.org/wiki/WGS84 